\title{
THREE ESSAYS ON BAYESIAN ANALYSIS OF NONLINEAR DSGE MODELS
}

\author{
A Dissertation \\ presented to \\ the Faculty of the Graduate School \\ at the University of Missouri-Columbia
}

In Partial Fulfillment

of the Requirements for the Degree

Doctor of Philosophy

by

Sanha Noh

Dr. Christopher Otrok, Dissertation Supervisor

MAY 2019 
The undersigned, appointed by the Dean of the Graduate School, have examined the dissertation entitled:

\section{THREE ESSAYS ON BAYESIAN ANALYSIS OF NONLINEAR DSGE MODELS}

presented by Sanha Noh, a candidate for the degree of Doctor of Philosophy and hereby certify that, in their opinion, it is worthy of acceptance.

Professor Christopher Otrok

Professor Shawn Ni

Professor J. Isaac Miller

Professor Scott Holan 


\section{ACKNOWLEDGMENTS}

My deep gratitude goes first to Professor Christopher Otrok, who expertly guided me through the arduous process of becoming a researcher in economics during my Ph.D. course. He provided me a great opportunity to work on a project for him. I could learn front-line research topics from the project. Without his invaluable advice and overwhelming support, I could not have completed my degree with a satisfactory result. Also, I would like to express my appreciation to my dissertation committee members; Dr. Shawn Ni, Dr. J. Isaac Miller, and Dr. Scott Holan. I especially thank and acknowledge Dr. Shawn Ni with all his guidance and invaluable mentoring in the classes of Research Workshop.

I would like to thank, without implication, Dr. Alessandro Rebucci, Dr. Andrew Foerster, and Dr. Gianluca Benigno for helpful suggestions while working for them as a research assistant. Dr. Alessandro Rebucci provided me helpful guidance and productive comments on my dissertation. I have learned the importance of positive thinking and belief in myself from him. I would also like to thank Professor Soosung Hwang spent his time to introduce me into the profession during my master's degree course.

My gratitude goes out to all the faculty members in the Department of Economics. They provided me useful direction and support throughout all the years in the program. I am especially indebted to Dr. Joe Haslag, Dr. Chao Gu, Dr. J. Isaac Miller, and Dr. David Kaplan for their teaching and guidance. Their dedicated teaching underlies the foundation of my dissertation and intuition on economics. In addition, I will not forget Dr. X.H. Wang, Lynne, and Linda for their helps from the beginning of my study in the U.S. I also thank my friends; Dr. Seoungyub Lee, Dr. Taehwan Kim, Dr. Tai Lee, Mr. Joonhong Ahn, Mr. Sanguk Kwon, and Mr. Heon Lee. I am mostly grateful to Dr. Kyungsik Nam and Dr. Ingul Baek for their relentless 
questions and supports on my researches.

This dissertation is the product of five years of effort and the culmination of too many years in school. I would like to thank my parents for giving me unstinting encouragement and support to continue my education. I would also like to thank my friends for being on my side and listening my predicament. Finally, my deepest thanks goes to my wife Heejin Park, to whom this dissertation is dedicated, for her patience, cheerfulness, and optimism throughout this process. The most precious gift that I was granted in the U.S. is my son, Aiden Noh. He fills my life with happiness and pleasure. 
TABLE OF CONTENTS

ACKNOWLEDGMENTS ................. ii

LIST OF TABLES $\ldots \ldots \ldots \ldots \ldots \ldots$ viii

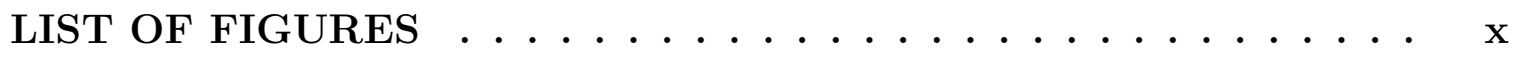

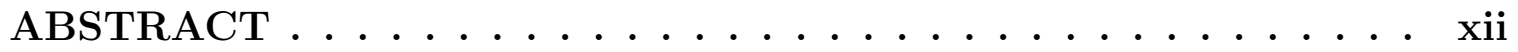

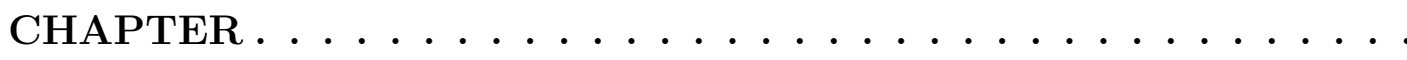

1 Posterior Inference on Parameters in a Nonlinear DSGE Model via Gaussian-Based Filters . . . . . . . . . . . . . . 1

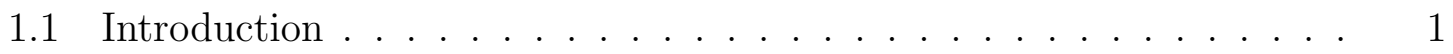

1.2 Bayesian Filtering and Likelihood Evaluation . . . . . . . . 6

1.2.1 The State Space Representation of DSGE Models . . . . . . 7

$1.2 .2 \quad$ Bayesian Filtering . . . . . . . . . . . . . . . 7

1.3 The Neoclassical Growth Model . . . . . . . . . . . . . . . . . 17

1.3 .1 The Model . . . . . . . . . . . . . . . . . . . . . . . 17

1.3.2 The Solution Method . . . . . . . . . . . . . . . . . 18

1.4 Posterior Inference on Parameters via MCMC . . . . . . . . . . . . 21

1.4.1 The Pseudo-Marginal MH for Parameter Estimation _. . . . 21

1.4.2 Discussion for Convergence of the Algorithm . . . . . . . . 22

1.4.3 Asymptotic Posterior Normality . . . . . . . . . . . . . 28

1.5 Monte Carlo Study . . . . . . . . . . . . . . . . . . . . 30

1.5 .1 The Priors . . . . . . . . . . . . . . . . . . . . 32

1.5.2 Results with 'Artificial' Data . . . . . . . . . . . . . . 34

1.6 Application to 'Real' Data . . . . . . . . . . . . . . . . . 46 
1.7 Conclusion . . . . . . . . . . . . . . . . . . . . . . . . . . . . . . . . . 49

2 House Price Uncertainty, Business Cycles, and Welfare . . . . 50

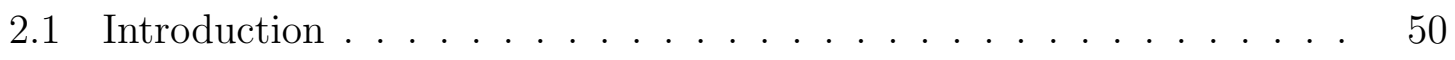

2.2 Uncertainty Transmission Mechanism . . . . . . . . . . . . 56

2.3 Evidence of Time-Varying House Price Uncertainty . . . . . . . . . 59

$2.3 .1 \quad$ House Price Uncertainty . . . . . . . . . . . . . . . . . . . 59

2.3 .2 Estimation . . . . . . . . . . . . . . . . . 62

2.3.3 Basic Empirical Regularities . . . . . . . . . . . . . . . 64

2.4 Empirical VAR Analysis $\ldots \ldots \ldots \ldots$

2.4.1 Benchmark MS-VAR Model . . . . . . . . . . . . . . 68

2.4.2 House Price Uncertainty Shocks and Economic Activity . . . . 70

2.5 Theoretical DSGE Model with a Housing Market . . . . . . . . . 74

2.5 .1 Model . . . . . . . . . . . . . . . . . . . . . . 75

2.5 .2 Solution and Calibration . . . . . . . . . . . . 83

2.5 .3 Estimation . . . . . . . . . . . . . . . . . . 84

2.6 Estimation Results $\ldots \ldots \ldots \ldots$

2.6.1 Parameter Estimates . . . . . . . . . . . . . . . . . 90 90

$2.6 .2 \quad$ Impulse Response Analysis . . . . . . . . . . . . . . . . . . 92

2.6.3 Source of House Price Uncertainty . . . . . . . . . . . . . . 94

2.6.4 Analytical Discussions . . . . . . . . . . . . . . . . . 99

2.6 .5 Additional Analysis . . . . . . . . . . . . . . . . 106

2.7 Welfare Analysis and Optimal Policy . . . . . . . . . . . . . 109

2.7.1 Uncertainty and Welfare . . . . . . . . . . . . . . . . 109

2.7.2 Uncertainty and Optimal Policy . . . . . . . . . . . . . 112

2.8 Conclusion . . . . . . . . . . . . . . . . . . . . . . . . 114 
3 Real Business Cycles in Emerging Countries: The Role of Nonlinearity ...................... 116

3.1 Introduction . . . . . . . . . . . . . . . . . 116

3.2 Models . . . . . . . . . . . . . . . . . . . . . . . . . . . . 118

3.2 .1 Benchmark Model . . . . . . . . . . . . . . . . . . . . . 118

$3.2 .2 \quad$ Financial Frictions . . . . . . . . . . . . . . . . . . . 122

3.3 Empirical methodology . . . . . . . . . . . . . . . . . . . . 123

3.4 Results . . . . . . . . . . . . . . . . . . . . . 125

3.4.1 Benchmark RBC model results . . . . . . . . . . . . . 126

3.4.2 Financial friction RBC model results . . . . . . . . . . . 127

3.4.3 Performance comparison: predictive marginal likelihood . . . . 128

3.4.4 Role of nonlinearity . . . . . . . . . . . . . . . . 130

3.5 Conclusion . . . . . . . . . . . . . . . . . . . . . . . . . 139

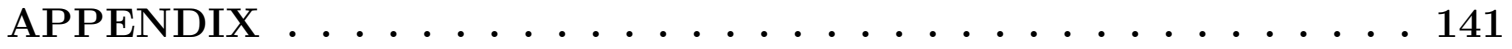

A Appendix to Chapter 1.................. 141

A.1 Central Difference Kalman filter . . . . . . . . . . . . . . . . 141

A.2 Binomial Gaussian mixture . . . . . . . . . . . . . 144

A.3 Proof . . . . . . . . . . . . . . . . . . 147

A.4 Second-order Approximation for Calibrated Parameters . . . . . . . . 149

A.5 Benchmark with stochastic volatility _ . . . . . . . . . 151

A.6 Data Sources over 1991Q1-2015Q3 _ . . . . . . . . . . . . 152

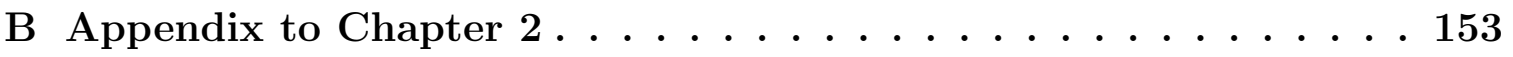

B.1 Particle MCMC . . . . . . . . . . . . . . . 153

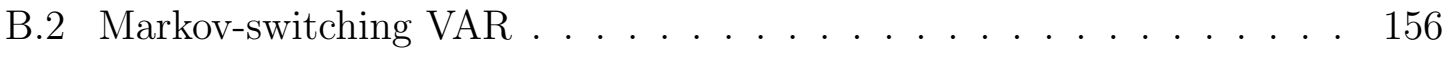

B.3 Third-order Approximation _. . . . . . . . . . . . . . 159 
B.4 Equilibrium Conditions . . . . . . . . . . . . . . . . . 160

B.5 Steady State . . . . . . . . . . . . . . . . . . . . . 165

B.6 Data Sources over 1977Q1-2008Q4 . . . . . . . . . . . . . 169

B.7 Uncertainty-Return Relationship . . . . . . . . . . . . . 170

B.8 Additional Analysis . . . . . . . . . . . . . . . . . . . . 174

Bibliography . . . . . . . . . . . . . . . . . 182

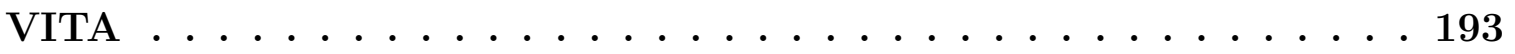




\section{LIST OF TABLES}

Table

Page

1.1 Gaussian-based filters . . . . . . . . . . . . . . . . . 3

1.2 Calibrated Parameters and Priors . . . . . . . . . . . . 33

1.3 Posterior Distribution: Benchmark Case . . . . . . . . . . 35

1.4 Logmarginal Likelihood: Benchmark Case . . . . . . . . . . . . 36

1.5 Posterior Distribution: Extremely Nonlinear Case . . . . . . . . . 39

1.6 Logmarginal Likelihood: Extremely Nonlinear Case . . . . . . . . . 40

1.7 Posterior Distribution: Nearly Linear Case . . . . . . . . . . . . . 41

1.8 Logmarginal Likelihood: Nearly Linear Case . . . . . . . . . . . . 42

1.9 Posterior Distribution: Benchmark with stochastic volatility . . . . 44

1.10 Logmarginal Likelihood: Benchmark with stochastic volatility . . . . 44

1.11 Posterior Distribution Real Data . . . . . . . . . . . . 48

1.12 Logmarginal Likelihood . . . . . . . . . . . . . . . . . . . . 48

1.13 Standard Deviations $\left(\sigma_{x}\right)$ (Real data vs Simulated data) $\ldots \ldots . .48$

2.1 Prior and Posterior Distribution . . . . . . . . . . . . 62

2.2 Forecast Error Variance Decomposition . . . . . . . . . . . . 73

2.3 Calibrated Parameters . . . . . . . . . . . . . . . . . 83

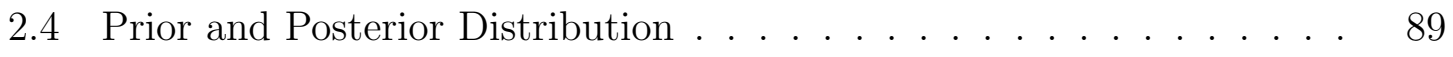

2.5 Estimated Parameter Values . . . . . . . . . . . . . . . . . . . 91 
2.6 Second Moments in the Model and Data . . . . . . . . . . . . . . . . 91

2.7 Variance Decomposition . . . . . . . . . . . . . . . . 95

2.8 House price uncertainty and Returns to housing demand . . . . . . . 104

2.9 Real-options channels . . . . . . . . . . . . . . . . . . 105

2.10 Compensating Variations: Baseline Model . . . . . . . . . . . . . . . 111

2.11 Welfare, Interest Rate Rule, and Uncertainty . . . . . . . . . . . . . . 114

3.1 Calibration ......................... 124

3.2 Benchmark RBC model . . . . . . . . . . . . . . . 126

3.3 Financial frictions model . . . . . . . . . . . . . . . . . . . 127

3.4 Subperiod standard deviations . . . . . . . . . . . . . . . . 130

3.5 Statistics . . . . . . . . . . . . . . . . . . 132

3.6 Variance Decomposition . . . . . . . . . . . . . . . 134

A.1 Posterior Distribution with $C A=0.1 \ldots \ldots$. . . . . . . 151

B.1 State-level evidence for financial risk and hedging effect . . . . . . . . 178 


\section{LIST OF FIGURES}

Figure $\quad$ Page

1.1 Posterior Distribution: Benchmark Case . . . . . . . . . . . . . 35

1.2 Posterior Distribution: Extremely Nonlinear Case . . . . . . . . . . . 39

1.3 Posterior Distribution: Nearly Linear Case . . . . . . . . . . . . . . . 41

1.4 Posterior Distribution: Benchmark with stochastic volatility . . . . . 43

1.5 Posterior Distribution Based on Real Data . . . . . . . . . . . . . . . 47

2.1 House Price Uncertainty . . . . . . . . . . . . . . . . . . . . 64

2.2 Cross Correlation . . . . . . . . . . . . . . . 66

2.3 Rolling Correlation . . . . . . . . . . . . . . . 66

2.4 Regime Indicator . . . . . . . . . . . . . . . . . . . . 70

2.5 Responses to House Price Uncertainty Shock in Regime1 (Expansionary) 72

2.6 Responses to House Price Uncertainty Shock in Regime2 (Recessionary) 72

2.7 Responses to a Joint Uncertainty Shock . . . . . . . . . . . . . . . 93

2.8 Responses to Supply-side Uncertainty Shock . . . . . . . . . . . . . . 98

2.9 Responses to Demand-side Uncertainty Shock . . . . . . . . . . . . 98

2.10 Welfare, LTV ratio, and Uncertainty . . . . . . . . . . . . 113

3.1 Predictive likelihoods . . . . . . . . . . . . . . . . . . . . . . 129

3.2 Measure of nonlinearity . . . . . . . . . . . . . . . . . . . . 131

3.3 Impulse responses to temporary prod. shocks . . . . . . . . . . . 136 
3.4 Impulse responses to permanent prod. shocks . . . . . . . . . . . . . 136

3.5 Impulse responses to temporary prod. shocks . . . . . . . . . . . . . 137

3.6 Impulse responses to permanent prod. shocks . . . . . . . . . . . . 137

3.7 Country premium shocks . . . . . . . . . . . . . . . . 138

3.8 Preference shocks . . . . . . . . . . . . . . . . . . . 138

3.9 Spending shocks . . . . . . . . . . . . . . . . . . . . . . . 138

A.1 Posterior Distribution with $C A=0.1 \ldots \ldots$. . . . . . 151

B.1 Impulse Responses to House Price Uncertainty Shock: Constant . . . 177

B.2 House Price Uncertainty . . . . . . . . . . . . . . . . . 177

B.3 Responses to Supply-side Uncertainty Shock: Role of price and wage stickiness . . . . . . . . . . . . . . . . . . . . . . . . . 179

B.4 Responses to Demand-side Uncertainty Shock: Role of price and wage stickiness . . . . . . . . . . . . . . . . . . . . . . . . 179

B.5 Responses to Supply-side Uncertainty Shock: Role of collateral constraints. . . . . . . . . . . . . . . . . . . 180

B.6 Responses to Demand-side Uncertainty Shock: Role of collateral constraints . . . . . . . . . . . . . . . . . . 180

B.7 Responses to Supply-side Uncertainty Shock: Role of perfect factor mobility . . . . . . . . . . . . . . . . . . . . . 181

B.8 Responses to Demand-side Uncertainty Shock: Role of perfect factor

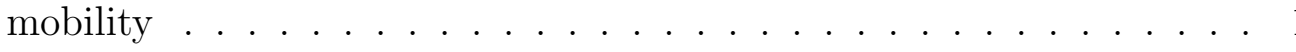




\section{ABSTRACT}

The 2008 financial crisis has highlighted the importance of nonlinear features of our economy including risks, uncertainty shocks, rare disasters, structural changes, zerolower bound, and occasionally binding constraints. Macroeconomists have tried to build nonlinear models to analyze these interesting features and take the models to the data. Dynamic Stochastic General Equilibrium (DSGE) model that essentially takes into account dynamic optimal decision making of households, firms, and government is one of the useful tools to deal with these issues. In the model, there are various random shocks causing the macroeconomic variables such as GDP, consumption, and investment to fluctuate over time. Above all things, the nonlinear approximation of the model allows us to capture the impact of risk on decision making. The focus of this dissertation is to provide a novel Bayesian estimation procedure for the estimation of nonlinear DSGE model and apply the proposed methodologies to analyze some nonlinear issues related to DSGE models.

In the first chapter, I study Gaussian-based filters within the pseudo-marginal Metropolis Hastings (PM-MH) algorithm for posterior inference on parameters in nonlinear DSGE models. I implement two Gaussian-based filters to evaluate the likelihood of a DSGE model solved to second and third order and embed them into the PM-MH: Central Difference Kalman filter (CDKF) and Gaussian mixture filter (GMF). The GMF is adaptively refined by splitting a mixture component into new mixture components based on Binomial Gaussian mixture. The overall results indicate that the estimation accuracy of the CDKF and the GMF is comparable to that of the particle filter $(\mathrm{PF})$, except that the CDKF generates biased estimates in the extremely nonlinear case. The proposed GMF generates the most accurate estimates

among them. I argue that the GMF with PM-MH can converge to the true and invariant distribution when the likelihood constructed by infinite Gaussian mixtures 
weakly converges to the true likelihood. In addition, I show that the Gaussian-based filters are more efficient than the PF in terms of effective computing time. Finally, I apply the method to real data.

In the second chapter, I investigate the asymmetric effects of house price uncertainty on economic activity over the business cycle. Preliminary evidence is uncovered with a Markov-switching vector autoregression (MS-VAR) model. The MS-VAR model finds that house price uncertainty shocks in expansionary regimes increase residential investment, housing prices, and mortgage debt, while the shocks in recessionary regimes have the opposite effects. These empirical results are further investigated in an estimated New-Keynesian DSGE model with a housing sector that allows for multiple structural volatility shocks. I show that uncertainty shocks to investmentspecific technology and inflation target reproduce the key empirical impulse responses in recessionary regimes from the MS-VAR. On the other hand, the responses to housing preference uncertainty shocks are consistent with the empirical impulse responses in expansionary regimes. I then show that the underlying mechanism behind the relationship between uncertainty and returns to owning houses is associated with a financial risk effect, a housing consumption hedging effect, and a debt risk effect. Finally, I find that the optimal Loan-to-Value (LTV) ratio is countercyclical with respect to housing preference uncertainty and monetary policy uncertainty, but it is procyclical with respect to uncertainty about investment-specific technology.

In the third chapter, I investigate a real business cycle (RBC) model for a small open economy by estimating the model solved up to second order. The higher order approximation more closely approximates the original model than the linear approximation. In this study, I evaluate the likelihood of the nonlinear model using the Gaussian mixture filter (GMF) and employ the GMF within the MCMC algorithm. From the estimation results of the quadratic approximation, I obtain the following implications for a small open economy: First, the quadratic RBC model with fi- 
nancial frictions does a good job at identifying the parameters of the nonstationary productivity shock process. Second, the observed data favor the quadratic benchmark RBC and financial-friction models over the linear models. Third, the quadratic RBC model with financial frictions does a better job at capturing serial correlations of the observed data than the linear model with financial frictions. Fourth, contrary to the linear model with financial frictions, a nonstationary productivity shock in the quadratic model plays an important role in explaining Argentine economic fluctuations. 


\section{Chapter 1}

\section{Posterior Inference on Parameters in a Nonlinear DSGE Model via Gaussian-Based Filters}

\section{$1.1 \quad$ Introduction}

The higher-order approximate solution of DSGE models is known to provide a better empirical fit and better characterize features of economic phenomenon in many situations. First, Fernández-Villaverde and Rubio-Ramírez (2005) show that the secondorder solution of a neoclassical growth model improves the marginal likelihood and matches the moments of real data better than the linearized model. Amisano and Tristani (2010) show that the second-order solution of a DSGE model generates more accurate inflation responses to shocks when the economy exhibits large deviations from the steady state. Second, the higher-order approximation captures risk which is important for asset pricing and welfare analysis (Kim and Kim, 2003; Rudebusch and Swanson, 2012). It also allows us to investigate the effect of uncertainty shocks and rare disasters on economic activities (Fernández-Villaverde et al., 2011; Andreasen, 2012; Born and Pfeifer, 2014a; Fernández-Villaverde et al., 2015; Fernández-Villaverde 
and Levintal, 2016).

The central difficulty in likelihood-based parameter estimation of nonlinear DSGE models is that the likelihood of the higher-order approximate solution cannot be evaluated in closed form. To circumvent this problem, likelihood-based estimation uses nonlinear Bayesian filters to approximate the likelihood function. Among them, the particle filter $(\mathrm{PF})$ has been widely used for the estimation of nonlinear DSGE models (see Fernández-Villaverde and Rubio-Ramírez, 2005, 2007; An and Schorfheide, 2007 for early applications). More importantly, the recent theoretical development of particle MCMC that makes use of the unbiased likelihood estimate produced by the PF allows us to embed the PF within MCMC algorithm (Andrieu et al., 2010).

Although the PF does track the entire distribution implied by the state space representation of DSGE models when infinite particles are allowed, it has disadvantages. First, they tend to require a large number of particles which grow exponentially with the state dimension so that they potentially have high computational cost (Pitt et al., 2012). Second, they potentially suffer from sample degeneracy so that only a few of particles will have significant weights, but all the other particles will have negligible weights. Although the resampling procedure is commonly implemented to address this problem by forcing particles to areas of high likelihood and abandoning low-weighted particles, it also creates sample impoverishment so that the state can collapse into a single particle while most of the particles with small weights are discarded (Chopin, 2004). These problems make the filter inefficient with a large variance of the likelihood estimate. Although the efficient particle filters that attempt to reduce the variance of the likelihood estimate have been proposed (Amisano and Tristani, 2010; Andreasen, 2011; DeJong et al., 2012; Hall et al., 2014; Herbst and Schorfheide, 2017), these are still perceived as concerns for the particle MCMC convergence.

As alternatives, the Extended Kalman filter (EKF) (Jazwinski, 1970), the Sigma- 
Table 1.1 Gaussian-based filters

\begin{tabular}{l|l|l}
\hline & Gaussian-based filters & Reference \\
\hline EKF & Extended Kalman filter & Jazwinski (1970) \\
\hline UKF & Uscented Kalman filter & Julier and Uhlmann (1997) \\
\hline CDKF & Central Difference Kalman filter & NøRgaard et al. (2000) \\
\hline CKF & Cubature Kalman filter & Arasaratnam and Haykin (2009) \\
\hline GMF & Gaussian mixture filter & Alspach and Sorenson (1971) \\
\hline
\end{tabular}

Point Kalman filter (SPKF) and the Gaussian mixture filter (GMF) can also be used to evaluate the likelihood of nonlinear and non-Gaussian state space models. The SPKF that propagates a set of deterministically chosen points (called sigma-points) through the measurement and state equations to construct the predictive and filtered density includes the Unscented Kalman filter (UKF) (Julier and Uhlmann, 1997), the Central Difference Kalman filter (CDKF) (NøRgaard et al., 2000), and the Cubature Kalman filter (CKF) (Arasaratnam and Haykin, 2009). Recently, Binning and Maih (2015) developed and applied the SPKF for the estimation of a nonlinear regime switching DSGE model. Moreover, based on the fact that any distribution can be obtained by mixing Gaussian distributions (Alspach and Sorenson, 1971, 1972), the GMF can approximately evaluate the exact likelihood when the number of mixture components is infinite. Since all of these filters approximate the likelihood using Gaussian distributions, we call them Gaussian-based filters. Table 1.1 summarizes a list of Gaussian-based filters.

Although the Gaussian-based approaches can generate approximation errors if the distribution is largely different from the Gaussian or if the number of Gaussian mixture components is not enough to capture the true distribution, it is computationally inexpensive and reasonably accurate to evaluate the likelihood. Andreasen (2013) documents that a quasi-maximum likelihood approach based on the CDKF provides more accurate estimation results than the PF. Binning and Maih (2015) shows a good performance of the SPKF in their regime switching application. In addition, those filters can be used to complement the PF by serving as a proposal 
density (Van Der Merwe and Wan, 2003). In a macro context, Amisano and Tristani (2010) use the EKF as a proposal distribution for the PF. Andreasen (2011) proposes the optimized proposal of the PF based on the CDKF. However, there have not been studies on directly employing Gaussian-based filters (such as EKF, SPKF, and GMF) within Bayesian procedures for posterior inference on parameters in nonlinear DSGE models.

In this study, we employ the CDKF as one alternative in the class of SPKF procedures and the GMF to evaluate the likelihood of nonlinear DSGE models and compare them with the PF. Both are embedded in the PM-MH algorithm for posterior inference on parameters in nonlinear DSGE models. As a second contribution, we perform a refined GMF that splits a Gaussian mixture component into new mixture components based on Binomial Gaussian mixture and merges them based on the Kullback-Leibler distance (Raitoharju et al., 2015). Although the Gaussian mixture approximation has been widely used to estimate stochastic volatility models (Kim et al., 1998; Primiceri, 2005; Omori et al., 2007; Justiniano and Primiceri, 2008), it has not been used in general nonlinear state space models due to the lack of guidance on how to avoid the potential degeneracy of the GMF. This paper is the first application of Binomial Gaussian mixture to deal with this issue when employing the GMF for the estimation of nonlinear DSGE models. In addition, we establish that the PMMH updates with the GMF leave its pseudo-posterior invariant and that, under the standard assumptions, the realizations of the PM-MH algorithm converge to the true and stationary density when the likelihood constructed by infinite Gaussian mixtures weakly converges to the true likelihood. Finally, we discuss properties of asymptotic pseudo-posterior normality implied by the Gaussian-based filters.

The goal of this study is to investigate the accuracy and computational efficiency of Gaussian-based filters for the evaluation of likelihood within the PM-MH algorithm. The paper answers the following key questions based on a simple neoclassical growth 
model. (1) How accurate are the Gaussian-based filters in terms of the estimation of parameters and state variables? (2) Are the Gaussian-based filters computationally efficient? (3) How well do the Gaussian-based filters with PM-MH explain data in terms of the marginal likelihood? We perform four different Monte Carlo exercises: the first three exercises are based on the second order approximation and the last exercise is based on the third order approximation with stochastic volatility. The first three exercises are divided into a benchmark case, an extremely nonlinear case, and a nearly linear case, depending on parameter values that control the Frisch labor elasticity and the standard deviations of structural shocks. High Frisch labor elasticity and large standard deviations tend to increase the size of nonlinearity terms. We calibrate the benchmark parameter values based on previous studies.

The main results indicate that the estimation accuracy of the CDKF and the GMF is comparable to that of the PF in the benchmark and nearly linear case solved to second order. Accuracy is based on the Root Mean Squared Errors (RMSEs) of filtered state variables and the Average Relative Biases (ARBs) of posterior means. In the benchmark model solved up to third order with stochastic volatility, the Gaussianbased filters produce relatively less accurate filtered state variables than they produce in the benchmark model solved up to second order without stochastic volatility, but they generate reasonably accurate point estimates for parameters based on posterior means and modes. Although the CDKF creates biased estimates for some parameters in the extremely nonlinear case solved to second order, the GMF complements the poor performance of the CDKF by mixing Gaussian distributions and shows better performance than the PF.

We show that the proposed Gaussian-based filters are much more efficient than the $\mathrm{PF}$ in terms of effective computing time. As interest among academics and practitioners in large models with many state variables increases, more efficient filtering algorithms for the likelihood evaluation is critical (Strid and Walentin, 2009; Herbst, 
2015). Moreover, the logmarginal likelihood implies that the GMF shows a better empirical fitting of the model to the data than the PF. Although the logmarginal likelihood of the CDKF is smaller than the GMF and the PF in the benchmark and extremely nonlinear case, it fits the data better than the PF in the nearly linear case. Finally, we apply the method to real data and test whether the CDKF and the GMF work for the real data. In this exercise, we solve the model up to second order and estimate the model on U.S. data over the 1991:Q1-2015:Q3. The results show that the CDKF and the GMF have the similar estimation results with those of the PF. In addition, all of the filters have the similar explanation of the real data.

The rest of the paper is organized as follows. In Section 2 we discuss three Bayesian filtering methods for the likelihood evaluation of a dynamic equilibrium economy. Section 3 introduces a simple neoclassical growth model for our application

and discusses model solution methods. Section 4 presents the PM-MH algorithm for posterior inference on parameters and discusses the convergence of the algorithm and the asymptotic pseudo-posterior normality. Section 5 reports our main findings. Section 6 concludes.

\subsection{Bayesian Filtering and Likelihood Evaluation}

In this section, we describe three Bayesian filtering methods which will be implemented within the PM-MH algorithm to evaluate the approximate likelihood of a nonlinear state space model. The first two filtering methods that we are mainly interested in consist of the CDKF and the GMF. Since both of them are based on Gaussian-based approximation, we call those methods Gaussian-based filters. As a benchmark for comparison, we will review the well known PF. We begin by reviewing the state space representation for a nonlinear DSGE model. Then we present the details of the filtering methods. 


\subsubsection{The State Space Representation of DSGE Models}

We consider a standard DSGE model which is flexible enough to describe a wide range of macroeconomic phenomenon. It can be represented by the following set of equations that implies the optimized behaviors of economic agents:

$$
E_{t} f\left(y_{t+1}, y_{t}, x_{t+1}, x_{t} ; \theta\right)=0,
$$

where $E_{t}$ is the expectation operator, $f$ is a set of known functions which represents optimized relationships between control variables $y_{t}$ with dimension $n_{y} \times 1$ and state variables $x_{t}$ with dimension $n_{x} \times 1$, and $\theta$ is a set of structural parameters in the model.

The class of models that we consider can be represented in the form of a state space model (see Schmitt-Grohé and Uribe, 2004 for illustration). The observation equation is given by

$$
y_{t}=g\left(x_{t} ; \theta\right)+v_{t},
$$

where $g(\cdot)$ determines the agents' decision rules and $v_{t} \sim$ i.i.d $\left(0, \mathrm{R}_{v}\right)$ with dimension $n_{v} \times 1$. The law of motion for the state variables is given by

$$
x_{t}=h\left(x_{t-1}, \eta_{t} ; \theta\right),
$$

where $h(\cdot)$ is the transition function which is determined by the one-period lagged state variables $x_{t-1}$ and the structural shocks $\eta_{t} \sim$ i.i.d $\left(0, \mathrm{R}_{\eta}\right)$ with dimension $n_{\eta} \times 1$.

\subsubsection{Bayesian Filtering}

To estimate the parameters in the nonlinear DSGE model, we need to evaluate the marginal posterior probability density $p\left(\theta \mid y_{1: T}\right) \propto p\left(y_{1: T} \mid \theta\right) p(\theta)$ of the parameters without knowing the normalization constant $p\left(y_{1: T}\right)$. Note that the state variables 
$x_{0: T}$ have been integrated out. Although the prior $p(\theta)$ is easy to evaluate, the difficult part is to evaluate the marginal likelihood $p\left(y_{1: T} \mid \theta\right)$. Ideally, the marginal likelihood can be calculated by using recursive computations of prediction density $p\left(x_{t+1} \mid y_{1: t} ; \theta\right)$ and filtering density $p\left(x_{t+1} \mid y_{1: t+1} ; \theta\right)$ (Jazwinski, 1970) as follows:

- Prediction Density

$$
p\left(x_{t+1} \mid y_{1: t} ; \theta\right)=\int p\left(x_{t+1} \mid x_{t} ; \theta\right) p\left(x_{t} \mid y_{1: t} ; \theta\right) d x_{t}
$$

- Filtering Density

$$
p\left(x_{t+1} \mid y_{1: t+1} ; \theta\right)=\frac{p\left(x_{t+1} \mid y_{1: t} ; \theta\right) p\left(y_{t+1} \mid x_{t+1} ; \theta\right)}{p\left(y_{t+1} \mid y_{1: t} ; \theta\right)}
$$

- Marginal Likelihood

$$
p\left(y_{1: T} \mid \theta\right)=\prod_{k=1}^{T} p\left(y_{k} \mid y_{1: k-1} ; \theta\right)
$$

where $p\left(y_{t} \mid y_{1: t-1} ; \theta\right)=\int p\left(y_{t} \mid x_{t} ; \theta\right) p\left(x_{t} \mid y_{1: t-1} ; \theta\right) d x_{t}$.

However, it is not easy to calculate the marginal likelihood as it involves an integration. Therefore, there have been various developments in approximate Bayesian filtering methods which can be used to approximate the likelihood function under the framework of nonlinear state space. Once we evaluate the marginal likelihood from the approximate filtering, we can directly embed the filtering scheme into the implementation of MCMC sampling for the parameter estimation. In this study, we will focus on the Gaussian-based filters (CDKF and GMF) with PM-MH for the parameter estimation and compare their performance with that of the PF with PM-MH in terms of estimation accuracy and computational efficiency. In the next subsection, those three approximate Bayesian filtering methods will be reviewed. 


\section{Central Difference Kalman Filter}

We employ the CDKF as one of the Gaussian-based filters. Since the CDKF based on the Stirlings's interpolation approximates the mean and covariance matrix of state variables using deterministically chosen sigma-points, we call it Sigma-Point Kalman filter (Binning and Maih, 2015). ${ }^{1}$ As described in NøRgaard et al. (2000), the CDKF approximates the unknown prediction and filtering density of unobserved state variables using a Gaussian distribution. It implies that it only requires estimating first two moments of the state variables. The estimated first and second moments by the CDKF accurately capture the true moments up to the 3rd order when the state vector is approximately Gaussian. For a non-Gaussian state vector, approximations are accurate up to 2nd order (Andreasen, 2013).

The filter for the equations (1.2) and (1.3) starts by combining the state vector $x_{t}$ and the exogenous disturbance $\eta_{t+1}$ into a single vector $\chi_{t}^{a}=\left[\begin{array}{ll}x_{t}^{\prime} & \eta_{t+1}^{\prime}\end{array}\right]^{\prime}$ with dimension $L$ where $\chi_{t \mid t}^{a}=\mathrm{E}\left[\chi_{t}^{a} \mid y_{1: t}\right], \chi_{t \mid t-1}^{a}=\mathrm{E}\left[\chi_{t}^{a} \mid y_{1: t-1}\right], P_{t \mid t}^{a}=\operatorname{Var}\left[\chi_{t}^{a} \mid y_{1: t}\right]$, and $P_{t \mid t-1}^{a}=\operatorname{Var}\left[\chi_{t}^{a} \mid y_{1: t-1}\right]$. The next step is to specify $2 L+1$ sigma-points in a way that they capture the full mean and covariance of the augmented state. The $i$ th sigmapoint $\chi_{i, t}^{a}=\left[\begin{array}{ll}\chi_{i, t}^{x \prime} & \chi_{i, t}^{\eta}{ }^{\prime}\end{array}\right]^{\prime}$ consists of the $\chi_{i, t}^{x}$ rows which contain state variables and the $\chi_{i, t}^{\eta}$ rows which contain exogenous disturbances. We define sigma-points for filtering

\footnotetext{
${ }^{1}$ We tested other Sigma-Point Kalman filters (UKF and CKF) for the likelihood evaluation and posterior inference on parameters in the application of our neoclassical growth model solved up to second and third order. However, the UKF, the CDKF, and the CKF show similar performance. The estimation results are available upon request.
} 
steps and prediction steps as follows:

$$
\begin{array}{ll}
\text { For prediction: } & \hat{\chi}_{0, t}^{a}=\chi_{t \mid t}^{a} \\
& \hat{\chi}_{i, t}^{a+}=\chi_{t \mid t}^{a}+h \mathrm{~S}_{i, t \mid t}^{a} \text { for } i=1 \ldots L \\
& \hat{\chi}_{i, t}^{a-}=\chi_{t \mid t}^{a}-h \mathrm{~S}_{i-L, t \mid t}^{a} \text { for } i=L+1 \ldots 2 L
\end{array}
$$

\section{For filtering: $\quad \bar{\chi}_{0, t}^{a}=\chi_{t \mid t-1}^{a}$}

$$
\begin{aligned}
& \bar{\chi}_{i, t}^{a+}=\chi_{t \mid t-1}^{a}+h \mathrm{~S}_{i, t \mid t-1}^{a} \text { for } i=1 \ldots L \\
& \bar{\chi}_{i, t}^{a-}=\chi_{t \mid t-1}^{a}-h \mathrm{~S}_{i-L, t \mid t-1}^{a} \text { for } i=L+1 \ldots 2 L,
\end{aligned}
$$

where $h=\sqrt{3}$ for a Gaussian distribution, $\mathrm{S}_{i, t \mid t}^{a}$ and $\mathrm{S}_{i, t \mid t-1}^{a}$ are the $i$ th column of the upper triangular Cholesky factorization of $P_{t \mid t}^{a}$ and $P_{t \mid t-1}^{a}$ respectively.

The sigma-points are propagated through the measurement and state equations to compute the prediction density and the filtering density by the following recursive algorithm:

1. Start from the initial filtered density $p\left(x_{1} \mid y_{1} ; \theta\right)$.

2. For each measurement $y_{t}$ for $t=2, \cdots, T$ :

- Prediction density: By integrating the state equations with the previous filtering density $p\left(x_{t-1} \mid y_{1: t-1} ; \theta\right)$, we get the following Gaussian approximation to the prediction density:

$$
p\left(x_{t} \mid y_{1: t-1} ; \theta\right) \approx N\left(\hat{x}_{t \mid t-1}, P_{t \mid t-1}^{x}\right)
$$

where $\hat{x}_{t \mid t-1}$ and $P_{t \mid t-1}^{x}$ are the predicted mean and the predicted covariance matrix, respectively.

- Filtering density: The predictions are then updated using the standard 
Kalman filter updating rule:

$$
p\left(x_{t} \mid y_{1: t} ; \theta\right) \approx N\left(\hat{x}_{t \mid t}, P_{t \mid t}^{x}\right)
$$

where $\hat{x}_{t \mid t}$ and $P_{t \mid t}^{x}$ are the filtered mean and the filtered covariance matrix, respectively.

3. Finally we can get the following conditional marginal likelihood,

$$
\hat{p}^{\mathrm{CDKF}}\left(y_{t} \mid y_{1: t-1} ; \theta\right)=N\left(\hat{y}_{t \mid t-1}, P_{t \mid t-1}^{y}\right),
$$

where $\hat{y}_{t \mid t-1}=\mathrm{E}\left[y_{t} \mid y_{1: t-1}\right]$ and $P_{t \mid t-1}^{y}=\operatorname{Var}\left[y_{t} \mid y_{1: t-1}\right]$.

A detailed description of the CDKF can be found in Appendix A.1 (See NøRgaard et al., 2000; Andreasen, 2013 for further details).

\section{Gaussian Mixture Filter}

The CDKF assumes that the states are well approximated by a Gaussian distribution. This assumption can fail in a nonlinear and non-Gaussian model, especially with multi-modal and heavy-tailed prediction and filtering distributions. The GMF addresses this problem by approximating distributions with a finite Gaussian mixture. This strategy is based on the fact that any continuous density can be obtained by infinitely mixing Gaussian densities in the sense of $L^{1}$ norm with the covariance matrix of every mixture component approaching zero (Alspach and Sorenson, 1971, 1972; Lo, 1972). This fact is based on the Wiener's theorem on approximation (Achieser, 2013),

$$
\int\left|p(x)-\sum_{l=1}^{L} w_{l} N\left(\mu_{l}, \Sigma_{l}\right)\right| d x \rightarrow 0 \quad \text { as } L \rightarrow \infty \text { and } \Sigma_{l} \rightarrow 0
$$

Given equations (1.2) and (1.3), we assume that the filtering density $p\left(x_{t-1} \mid y_{1: t-1} ; \theta\right)$, the density for exogenous shocks $p\left(\eta_{t}\right)$, and the density for measurement errors $p\left(v_{t}\right)$ 
are approximated by mixing $L$-components of Gaussian $\left(\approx \sum_{l=1}^{L} \alpha_{t-1}^{[l]} N\left(x_{t-1} ; \mu_{t-1}^{[l]}, P_{t-1}^{[l]}\right)\right)$, $M$-components of Gaussian $\left(\approx \sum_{m=1}^{M} \beta_{t}^{[m]} N\left(\eta_{t} ; \mu_{\eta_{t}}^{[m]}, P_{\eta_{t}}^{[m]}\right)\right)$, and $N$-components of Gaussian $\left(\approx \sum_{n=1}^{N} \gamma_{t}^{[n]} N\left(v_{t} ; \mu_{v_{t}}^{[n]}, P_{v_{t}}^{[n]}\right)\right)$, respectively. In this study, the initial number of mixture components of $p\left(x_{1} \mid y_{1} ; \theta\right)$ is two and split into new mixture components over iterations. We set $M=1$ and $N=1$ because, in a simulation study, we will assume Gaussian distributions on the structural shocks and the measurement errors. ${ }^{2}$

The prediction and filtering density also can be expressed by the mixture of Gaussian densities:

- Prediction density

$$
p\left(x_{t} \mid y_{1: t-1} ; \theta\right) \approx \sum_{l^{\prime}=1}^{L^{\prime}} w_{t}^{\left[l^{\prime}\right]} N\left(x_{t} ; \tilde{\mu}_{t}^{\left[l^{\prime}\right]}, \tilde{P}_{t}^{\left[l^{\prime}\right]}\right)
$$

- Filtering density

$$
p\left(x_{t} \mid y_{1: t} \theta\right) \approx \sum_{l^{\prime \prime}=1}^{L^{\prime \prime}} w_{t}^{\left[l^{\prime \prime}\right]} N\left(x_{t} ; \mu_{t}^{\left[l^{\prime \prime}\right]}, P_{t}^{\left[l^{\prime \prime}\right]}\right)
$$

where $L^{\prime}=L M$ and $L^{\prime \prime}=L M N$.

The mean and covariance matrix of $p\left(x_{t} \mid y_{1: t-1} ; \theta\right)$ and $p\left(x_{t} \mid y_{1: t} ; \theta\right)$ are computed by using the Central Difference Kalman filtering method. The mixing weights are updated by $w_{t}^{\left[l^{\prime}\right]}=\frac{\alpha_{t-1}^{[l]} \beta_{t}^{[m]}}{\sum_{l=1}^{L} \sum_{m=1}^{M} \alpha_{t-1}^{[l]} \beta_{t}^{[m]}}$ and $w_{t}^{\left[l^{\prime \prime \prime}\right]}=\frac{w_{t}^{\left[l^{\prime}\right]} \gamma_{t}^{[n]} S_{t}^{[n]}}{\sum_{l^{\prime}=1}^{L^{\prime}} \sum_{n=1}^{N} w_{t}^{\left[l^{\prime}\right]} \gamma_{t}^{[n]} S_{t}^{[n]}}$ where $\alpha_{t-1}^{[l]}, \beta_{t}^{[m]}$, and $\gamma_{t}^{[n]}$ denote the mixture weights of the filtering density at time $t-1$, the density for exogenous shocks at time $t$, and the density for measurement errors at time $t$, respectively. $S_{t}^{[n]}=p\left(y_{t} \mid x_{t} ; \theta\right)$ evaluated at $x_{t}=\tilde{\mu}_{t}^{\prime \prime}$ for the current observation $y_{t}$.

\footnotetext{
${ }^{2}$ We can apply the GMF to models with non-Gaussian structural shocks and measurement errors by estimating the Gaussian mixture densities of the structural shocks and the measurement errors. The Gaussian mixture densities can be estimated using an Expectation Maximization (EM) algorithm (McLachlan and Peel, 2004.
} 
The conditional marginal likelihood is obtained as follows:

$\hat{p}^{\mathrm{GMF}}\left(y_{t} \mid y_{1: t-1} ; \theta\right)=\int p\left(y_{t} \mid x_{t} ; \theta\right) p\left(x_{t} \mid y_{1: t-1} ; \theta\right) d x_{t}=\sum_{l^{\prime}=1}^{L^{\prime}} \sum_{n=1}^{N} w_{t}^{\left[l^{\prime}\right]} \gamma_{t}^{[n]} N\left(\hat{y}_{t \mid t-1}^{\left[l^{\prime} \times n\right]}, P_{t \mid t-1}^{y,\left[l^{\prime} \times n\right]}\right)$

The GMF performs well without potential degeneracy of mixture components when the mixture components have well-dispersed component means, small component covariance matrices, and balanced component weights (Niemi and West, 2010). To keep these properties while doing the GMF, we apply a splitting procedure on $p_{l^{\prime \prime}}\left(x_{t} \mid y_{1: t} ; \theta\right)$ for each mixture component based on the Binomial Gaussian mixture (Raitoharju et al., 2015). The parameters for the Binomial Gaussian mixture are chosen such that the mean and covariance of $p_{l^{\prime \prime}}\left(x_{t} \mid y_{1: t} ; \theta\right)$ are preserved. Different from the work of Raitoharju et al. (2015) who apply the Unscented Kalman filter, we apply the CDKF to the GMF and implement the splitting procedure to the filtering density. Specifically, for every iteration, when the size of nonlinearity measure of the measurement equation is above a pre-specified threshold $\left(\eta_{\text {limit }}\right)$, we approximate each mixture component of $p_{l^{\prime \prime}}\left(x_{t} \mid y_{1: t} ; \theta\right)$ by a weighted sum of Gaussian distributions of which component means, component covariance matrices, and component weights are selected using the Binomial Gaussian mixture. The resulting density function from the Binomial Gaussian mixture converges to the original Gaussian distribution (Raitoharju et al., 2015). We use a nonlinearity measure proposed by Raitoharju and Ali-Loytty (2012) after a normalization by dividing it by measurement error variance. The measure is based on the comparison between the Extended Kalman filter and the Second Order Gaussian filter:

$$
N L^{l}=\frac{\operatorname{trace}\left(Q^{l} Q^{l}\right)}{h^{4}}
$$


where $l$ indicates the $l$ th measurement equation. $Q^{l}$ is defined as

$$
Q_{[c, d]}^{l}= \begin{cases}g_{l}\left(\chi_{t \mid t-1}^{a}+h \mathrm{~S}_{i, t \mid t-1}^{a}\right)+g_{l}\left(\chi_{t \mid t-1}^{a}-h \mathrm{~S}_{i, t \mid t-1}^{a}\right)-2 g_{l}\left(\chi_{t \mid t-1}^{a}\right) & c=d \\ \frac{1}{2}\left[g_{l}\left(\chi_{t \mid t-1}^{a}+h \mathrm{~S}_{i, t \mid t-1}^{a}+h \mathrm{~S}_{i, t \mid t-1}^{a}\right)+g_{l}\left(\chi_{t \mid t-1}^{a}-h \mathrm{~S}_{i, t \mid t-1}^{a}-h \mathrm{~S}_{i, t \mid t-1}^{a}\right)-\right. & \\ \left.2 g_{l}\left(\chi_{t \mid t-1}^{a}\right)-Q_{[c, c]}^{l}-Q_{[d, d]}^{l}\right] & c \neq d\end{cases}
$$

The above notations come from the definition of the sigma-points discussed in the equation (1.7) for the CDKF. When splitting a mixture component, the total number of components is chosen such that the nonlinearity is reduced to $\eta_{\text {limit }}$. If the total number of mixture components in the splitting procedure exceeds a given threshold $\left(m_{\text {limit }}\right)$, we set the number of components to $m_{\text {limit }}$ and the component covariance matrix is chosen such that the nonlinearity is minimized. The details of the splitting procedure are provided in Appendix A.2. Since the splitting procedure might create a large number of mixture components, we implement a component reduction procedure. When the number of components exceeds a certain criterion $\left(m_{\text {reduce }}\right)$ or the Kullback-Leibler $(\mathrm{KL})$ discrimination measure between two mixture components exceeds a given threshold $\left(B_{\text {limit }}\right),{ }^{3}$ the component pair is merged such that the mean and covariance of the mixture is preserved. We use the KL measure proposed in Runnalls (2007). Since the measurement errors are not correlated with each other, we calculate the nonlinearity measure for each measurement equation and separately process the updating procedure of the CDKF over each measurement equation following Raitoharju et al. (2015).

\footnotetext{
${ }^{3}$ Runnalls (2007) proposes the following KL-based discrimination measure:

$$
\begin{aligned}
B_{i, j} & =\frac{1}{2}\left[\left(w_{i}+w_{j}\right) \log \operatorname{det} P_{i, j}-w_{i} \log \operatorname{det} P_{i}-w_{j} \log \operatorname{det} P_{j}\right] \\
P_{i, j} & =\frac{w_{i} P_{i}+w_{j} P_{j}}{w_{i}+w_{j}}+\left(\mu_{i}-\mu_{j}\right)\left(\mu_{i}-\mu_{j}\right)^{\prime}
\end{aligned}
$$
}

where $\mu_{i}$ is the (predictive or filtered) mean for component $i, w_{i}$ is the weight for mixture component $i$, and $P_{i}$ is the (predictive or filtered) covariance matrix for component $i$. 


\section{Particle Filter}

Although the GMF is very flexible, it cannot accommodate any possible densities in a finite mixture of Gaussian densities. As an alternative method, the particle-based sampling filter can be used to approximate the marginal likelihood for a nonlinear and non-Gaussian state space. It uses a set of weighted particles without any assumption on the distributional form so that it is nonparametric. Although it generates asymptotically exact likelihood by tracking the entire distribution, it can be inefficient when the measurement error in the model is very small or the filtering distribution has heavy tails (possibly because outliers happen in the data) (Amisano and Tristani, 2010). For the comparison between the already proposed Gaussian-based filters and the PF, we use the optimized central difference particle filter suggested by Andreasen (2011). ${ }^{4}$ Since it improves the accuracy and efficiency of the PF even in the existence of sample degeneracy and impoverishment, it is a good comparison target. As usual in the PF, we begin with the following filtering state density:

$$
p\left(x_{0: t+1} \mid y_{1: t+1} ; \theta\right)=p\left(x_{0: t} \mid y_{1: t} ; \theta\right) p\left(x_{t+1} \mid x_{0: t}, y_{1: t+1} ; \theta\right) .
$$

Since we do not know the exact form of the filtering density $p\left(x_{0: t+1} \mid y_{1: t+1} ; \theta\right)$, it is not possible to directly generate samples from $p\left(x_{0: t+1} \mid y_{1: t+1} ; \theta\right)$. The PF solve this problem by using importance sampling based on a proposal distribution $\pi\left(x_{0: t+1} \mid y_{1: t+1}\right)=$ $\pi\left(x_{0: t} \mid y_{1: t}\right) \pi\left(x_{t+1} \mid x_{0: t}, y_{1: t+1}\right)$ so that the performance of the PF inherently depends on the proposal distribution. The support of the proposal density should include that of filtering density. Based on the PF, $p\left(x_{0: t} \mid y_{1: t}\right) \approx \sum_{i=1}^{N} w\left(x_{t}^{[i]}\right) \delta\left(x_{0: t}-x_{0: t}^{[i]}\right)$ where $\delta($.$) is the Dirac delta function and \left\{x_{0: t}^{[i]}\right\}_{i=1}^{N}$ are samples from the proposal distribution $\pi\left(x_{0: t} \mid y_{1: t}\right)$. The optimized central difference particle filter is implemented by the following steps:

\footnotetext{
${ }^{4}$ We greatly thank Martin M. Andreasen for making publicly available his codes for the optimized central difference particle filter.
} 
1. Draw initial particles $x_{0}^{[i]}$ from $p\left(x_{0}\right)$ and set $w\left(x_{0}^{[i]}\right)=1 / N$ for $i=1,2, \ldots, N$.

2. Draw a large number of samples of endogenous state variables $\left\{x_{1, t}^{[i]}\right\}_{i=1}^{N}$ from the following transition distribution,

$$
x_{1, t}^{[i]} \sim p\left(x_{1, t} \mid \hat{x}_{t-1}^{[i]} ; \theta\right) \text { for } i=1,2, \ldots, N \text {. }
$$

3. Draw a large number of samples of exogenous state variables $\left\{x_{2, t}^{[i]}\right\}_{i=1}^{N}$ by using the mean $\hat{x}_{2, t}^{C D K F}$ and covariance matrix $\hat{S}_{x_{2}, t}^{C D K F}$ of updating distribution through the CDKF,

$$
x_{2, t}^{[i]}=\hat{x}_{2, t}^{C D K F}+\gamma_{t} \hat{S}_{x_{2}, t}^{C D K F} \varepsilon_{t}^{[i]} \text { for } i=1,2, \ldots, N
$$

where $\gamma_{t}$ is determined by minimizing the variance of the sampling weights in each $t$. The number of particles $N_{o p t}$ for the optimization is set to 300 . If the effective sample size $\hat{N}_{e f f, t}=\frac{1}{\sum_{i=1}^{N}\left(\tilde{w}\left(\gamma_{t}\right)_{t}^{[i]}\right)^{2}}$ is below 300, we re-optimize using all particles $N$.

4. Evaluate the importance weights,

$$
w\left(x_{t}^{[i]}\right)=w\left(x_{t-1}^{[i]}\right) \frac{p\left(y_{t} \mid x_{t}^{[i]} ; \theta\right) p\left(x_{t}^{[i]} \mid x_{t-1}^{[i]} ; \theta\right)}{N\left(x_{t}^{[i]} \mid \hat{x}_{2, t}^{C D K F}, \gamma_{t}^{2} \hat{S}_{x_{2}, t}^{C D K F} \hat{S}_{x_{2}, t}^{C D K F \prime}\right)} \text { for } i=1,2, \ldots, N
$$

The conditional marginal likelihood is obtained from the following equation:

$$
\hat{p}^{\mathrm{PF}}\left(y_{t} \mid y_{1: t-1} ; \theta\right)=\sum_{i=1}^{N} w\left(x_{t}^{[i]}\right)
$$

5. Resample with replacement from $\left\{x_{0: t}^{[i]}\right\}_{i=1}^{N}$ with probabilities $\tilde{w}_{t}^{[i]}=\frac{w\left(x_{t}^{[i]}\right)}{\sum_{i=1}^{N} w\left(x_{t}^{[i]}\right)}$ and set $w\left(x_{t}^{[i]}\right)=1 / N$ for $i=1,2, \ldots, N$. The resampled particles are denoted by $\left\{\hat{x}_{0: t}^{[i]}\right\}_{i=1}^{N}$. The filtered mean is calculated by $\hat{x}_{t}=1 / N \sum_{i=1}^{N} \hat{x}_{t}^{[i]}$.

A complete description of the optimized PF can be found in Andreasen (2011). 


\subsection{The Neoclassical Growth Model}

\subsubsection{The Model}

The model used in this study is a stochastic neoclassical growth model augmented by choice of leisure. The model is based on Brock and Mirman (1972). The reasons for choosing this model are twofold. First, this model is the building block of the standard dynamic general equilibrium models. We can extend the model by employing real and nominal rigidities. By implementing our estimation procedures in the most representative model, we can test their validity and apply them to more complicated models. Second, the neoclassical growth model is relatively simple to implement an estimation procedure even in the case that the nonlinear approximation solution is applied to the model. Since we are doing multiple estimation for the Monte Carlo evaluation, the speed for the estimation is essential.

Preferences: There are infinitely-lived representative households with preferences over goods and leisure. They maximize the expected sum of discounted utilities,

$$
U=E_{0} \sum_{t=0}^{\infty} \beta^{t}\left\{\log \left(c_{t}\right)+\psi \frac{\left(1-h_{t}\right)^{(1-\sigma)}-1}{1-\sigma}\right\}
$$

where $c_{t}$ is consumption level, $h_{t}$ is labor supply, $\psi$ is a strictly positive preference parameter, and $\sigma$ controls the Frisch labor supply elasticity $(\sigma \gamma)^{-1}$ where $\gamma=\frac{\bar{h}}{1-\bar{h}}$. The preference parameter $\psi$ is pinned down by steady state equations $\left(\psi=\frac{\alpha / \bar{c} \bar{k}^{1-\alpha} \bar{h}^{\alpha-1}}{(1-\bar{h})^{-\sigma}}\right)$. The steady state level of labor supply $\bar{h}$ is set to 0.3 . The utility from the leisure $1-h_{t}$ which is represented by a CRRA function enters separably in the utility function. This preference representation is based on King et al. (1988a,b). They provide a particular form of utility function that allows for balanced growth path. 
Technology: The goods are produced by the following technology,

$$
y_{t}=z_{t} k_{t}^{1-\alpha} h_{t}^{\alpha}
$$

where $\alpha \in(0,1), k_{t}$ represents physical capital, $h_{t}$ is labor, and $z_{t}$ denotes stationary technology shocks. The stationary technology shocks follow $\ln z_{t+1}=\rho_{z} \ln z_{t}+\eta_{z, t+1}$ with $\eta_{z, t+1} \sim i . i . d N\left(0, \sigma_{z}\right)$.

\section{Law of Motion for Capital:}

$$
k_{t+1}=\mu_{t} i_{t}+(1-\delta) k_{t}
$$

where $\mu_{t}$ denotes an investment specific technology shock. It follows $\ln \mu_{t+1}=$ $\rho_{\mu} \ln \mu_{t}+\eta_{\mu, t+1}$ with $\eta_{\mu, t+1} \sim i . i . d N\left(0, \sigma_{\mu}\right)$.

\section{Resource Constraint:}

$$
c_{t}+k_{t+1}=z_{t} k_{t}^{1-\alpha} h_{t}^{\alpha}+(1-\delta) k_{t}
$$

From the above optimization problem, we can get the first-order conditions related to the optimal behavior of $c_{t}, h_{t}, i_{t}$, and $k_{t+1}$. By solving the difference equations, we can get policy functions for $c_{t}, h_{t}$, and $i_{t}$ that depend on capital, technology, and investment-specific technology.

\subsubsection{The Solution Method}

We solve the model using second and third order perturbation methods which use second and third order Taylor series expansions around the nonstochastic steady state. ${ }^{5}$ It is relatively faster than other solution methods such as the value function

\footnotetext{
${ }^{5}$ The higher-order approximations create explosive sample paths because the higher-order terms generate unstable steady states. To deal with this problem, it is recommended to apply pruning
} 
iteration and projection method. Since the estimation procedure requires the solution result over every iteration for the evaluation of the likelihood, we use this method for computational convenience. We apply the codes by Schmitt-Grohé and Uribe (2004) to solve the model up to second-order terms and use the codes in Andreasen (2012) to compute third-order terms.

The second-order approximation can be represented by the following state space system:

$$
\begin{aligned}
& y_{t}=\frac{1}{2} g_{\sigma \sigma}+G_{x}\left(x_{t}\right)+\frac{1}{2} G_{x x}\left(x_{t} \otimes x_{t}\right)+\mathrm{R}_{v}^{1 / 2} v_{t} \\
& x_{t+1}=\frac{1}{2} h_{\sigma \sigma}+H_{x}\left(x_{t}\right)+\frac{1}{2} H_{x x}\left(x_{t} \otimes x_{t}\right)+\sigma \mathrm{R}_{\eta}^{1 / 2} \eta_{t+1}
\end{aligned}
$$

where $\mathrm{R}_{\eta}^{1 / 2}=\left[\begin{array}{cc}0 & 0 \\ \sigma_{z} & 0 \\ 0 & \sigma_{\mu}\end{array}\right], \mathrm{R}_{v}^{1 / 2}=\left[\begin{array}{ccc}\sigma_{m 1} & 0 & 0 \\ 0 & \sigma_{m 2} & 0 \\ 0 & 0 & \sigma_{m 3}\end{array}\right], \eta_{t+1} \sim i . i . d N\left(0, \mathrm{I}_{2}\right)$, and $v_{t} \sim i . i . d$ $N\left(0, \mathrm{I}_{3}\right) . x_{t}$ includes state variables (predetermined variables), $y_{t}$ is a vector of control variables (nonpredetermined variables), and $\eta_{t}$ is a vector of structural shocks for technology and investment-specific technology shocks. Specifically, $x_{t}=\left[k_{t}, z_{t}, \mu_{t}\right]^{\prime}$, $y_{t}=\left[c_{t}, i_{t}, h_{t}\right]^{\prime}$, and $\eta_{t}=\left[\eta_{z, t}, \eta_{\mu, t}\right]^{\prime}$. All constant terms and coefficients, $g_{\sigma \sigma}, G_{x}, G_{x x}$, $h_{\sigma \sigma}, H_{x}$, and $H_{x x}$ that are functions of structural parameters are $n y \times 1, n y \times n x$, $n x \times n x n y, n x \times 1, n x \times n x$, and $n x \times n x^{2}$ matrices, respectively. The scalar $\sigma$ is the perturbation parameter.

We also consider the neoclassical growth model with stochastic volatility. The stochastic volatility is introduced into the technology shock process:

$$
\log z_{t+1}=\rho_{z} \log z_{t}+\sigma_{z, t+1} \eta_{z, t+1},
$$

scheme that omits terms of higher-order effects (Kim et al., 2008). However, the simulated sample paths without pruning are stable and are not much different from those obtained with pruning in our case. Moreover, the estimation results without pruning are consistent with those with pruning so that we will report the results obtained without pruning. The estimation results obtained with pruning are available upon request. 
where $\eta_{z, t+1} \sim$ i.i.dN $\left(0, \sigma_{z}\right)$. The stochastic volatility is expressed as $\mathrm{AR}(1)$ process:

$$
\log \sigma_{z, t+1}=\rho_{\sigma_{z}} \log \sigma_{z, t}+\eta_{\sigma_{z}, t+1}
$$

where $\eta_{\sigma_{z}, t+1} \sim i . i . d N\left(0, \sigma_{\sigma_{z}}\right)$.

The model is solved up to third order as follows:

$$
\begin{aligned}
& y_{t}=\frac{1}{2} g_{\sigma \sigma}+G_{x}\left(x_{t}\right)+\frac{1}{2} G_{x x}\left(x_{t} \otimes x_{t}\right)+\frac{1}{6} G_{x x x}\left(\left(x_{t} \otimes x_{t}\right) \otimes x_{t}\right)+\frac{3}{6} g_{\sigma \sigma x}\left(x_{t}\right) \\
& +\frac{1}{6} g_{\sigma \sigma \sigma}+\mathrm{R}_{v}^{1 / 2} v_{t} \\
& x_{t+1}=\frac{1}{2} h_{\sigma \sigma}+H_{x}\left(x_{t}\right)+\frac{1}{2} H_{x x}\left(x_{t} \otimes x_{t}\right)+\frac{1}{6} H_{x x x}\left(\left(x_{t} \otimes x_{t}\right) \otimes x_{t}\right)+\frac{3}{6} h_{\sigma \sigma x}\left(x_{t}\right) \\
& +\frac{1}{6} h_{\sigma \sigma \sigma}+\mathrm{R}_{\eta}^{1 / 2} \eta_{t+1}
\end{aligned}
$$

where $\mathrm{R}_{\eta}^{1 / 2}=\left[\begin{array}{cccc}0 & 0 & 0 & 0 \\ 0 & \sigma_{\mu} & 0 & 0 \\ 0 & 0 & \sigma_{z} & 0 \\ 0 & 0 & 0 & \sigma_{\sigma_{z}}\end{array}\right], \mathrm{R}_{v}^{1 / 2}=\left[\begin{array}{ccc}\sigma_{m 1} & 0 & 0 \\ 0 & \sigma_{m 2} & 0 \\ 0 & 0 & \sigma_{m 3}\end{array}\right], \eta_{t+1} \sim i . i . d N\left(0, \mathrm{I}_{4}\right)$, and $v_{t} \sim$ i.i.dN $\left(0, \mathrm{I}_{3}\right) . \quad x_{t}=\left[k_{t}, \mu_{t}, \lambda_{t}, \sigma_{z, t}\right]^{\prime}, y_{t}=\left[c_{t}, i_{t}, h_{t}\right]$, and $\eta_{t}=\left[\eta_{\mu, t}, \eta_{z, t}, \eta_{\sigma_{z}, t}\right]$. $\lambda_{t}$ is an auxiliary variable for the specification of stochastic volatility. All constant terms and coefficients, $g_{\sigma \sigma}, G_{x}, G_{x x}, G_{x x x}, g_{\sigma \sigma x}, g_{\sigma \sigma \sigma}, h_{\sigma \sigma}, H_{x}, H_{x x}, H_{x x x}, h_{\sigma \sigma x}$, and $h_{\sigma \sigma \sigma}$ that are functions of structural parameters are $n y \times 1, n y \times n x, n x \times n x n y$, $n x \times n x n x n y, n y \times n x, n y \times 1, n x \times 1, n x \times n x, n x \times n x^{2}, n x \times n x^{3}, n x \times n x$, and $n x \times 1$ matrices, respectively. 


\subsection{Posterior Inference on Parameters via MCMC}

\subsubsection{The Pseudo-Marginal MH for Parameter Estimation}

In this section, we review how to incorporate the marginal likelihood function into the Bayesian estimation algorithm. The main inference step is to construct the posterior density of parameters given the data that is proportional to the marginal likelihood times the prior density. Since the posterior distribution and the marginal likelihood in a nonlinear approximate solution of DSGE model do not have closed form expressions, we use the pseudo-marginal $\mathrm{MH}$ algorithm to draw from the posterior distribution, implying that the pseudo-likelihood within the MH scheme is evaluated by nonlinear filtering methods. The specific algorithm to draw $\theta$ from the posterior $p\left(\theta \mid y_{1: T}\right)$ is as follows:

Let $p\left(\theta \mid y_{1: T}\right)$ be the unnormalized posterior probability density:

$$
p\left(\theta \mid y_{1: T}\right) \propto p\left(y_{1: T} \mid \theta\right) p(\theta)
$$

1. Draw $\theta_{0}$ from an initial distribution $p_{0}(\theta)$, set $S_{0}$ to be the lower triangular Cholesky factor of the initial covariance matrix $\Sigma_{0}$ and then, evaluate the initial pseudo-marginal likelihood $\hat{p}^{k}\left(y_{1: T} \mid \theta_{0}\right)$ for $k \in\{\mathrm{CDKF}, \mathrm{GMF}, \mathrm{PF}\}$.

2. Sample a candidate using $q\left(\theta^{*} \mid \theta_{i-1}\right)$. $\left(\theta^{*}=\theta_{i-1}+S_{i-1} \epsilon_{i}\right.$ where $\epsilon_{i} \sim N(0, I)$ in this study because we use a random-walk-based $\mathrm{MH}$ algorithm for the estimation of DSGE models.)

3. Evaluate the pseudo-marginal likelihood $\hat{p}^{k}\left(y_{1: T} \mid \theta^{*}\right)$.

4. Calculate the acceptance probability,

$$
\hat{\alpha}^{k}\left(\theta^{*} \mid \theta_{i-1}\right)=\min \left\{1, \frac{\hat{p}^{k}\left(y_{1: T} \mid \theta^{*}\right) p\left(\theta^{*}\right) q\left(\theta_{i-1} \mid \theta^{*}\right)}{\hat{p}^{k}\left(y_{1: T} \mid \theta_{i-1}\right) p\left(\theta_{i-1}\right) q\left(\theta^{*} \mid \theta_{i-1}\right)}\right\} .
$$


5. Sample a uniform random variable $\mathrm{u}$ from the uniform distribution $\mathrm{U}(0,1)$

6. If $\mathrm{u} \leq \hat{\alpha}^{k}\left(\theta^{*} \mid \theta_{i-1}\right)$, set $\theta_{i}=\theta^{*}$. Otherwise set $\theta_{i}=\theta_{i-1}$.

7. (Optional) Adjust the covariance matrix of the Gaussian proposal satisfying the following equation,

$$
S_{i} S_{i}^{T}=S_{i-1}\left(\mathrm{I}+i^{-\gamma}\left(\hat{\alpha}^{k}\left(\theta^{*} \mid \theta_{i-1}\right)-\bar{\alpha}_{*}\right) \frac{\epsilon_{i} \epsilon_{i}^{T}}{\left\|\epsilon_{i}\right\|^{2}}\right) S_{i-1}^{\prime},
$$

where $\gamma \in(1 / 2,1]$ (Vihola, 2012)

8. Set $\mathrm{i}=\mathrm{i}+1$ and go to step 2 until the desired number of samples has been generated.

We adaptively adjust the proposal covariance matrix $S_{i}$ to achieve an acceptance rate around $30 \%$.

\subsubsection{Discussion for Convergence of the Algorithm}

For the parameter estimation in a general state space representation, our main interest is to sample parameters from the marginal posterior density $p\left(\theta \mid y_{1: T}\right) \propto p\left(y_{1: T} \mid \theta\right) p(\theta)$ of $p\left(\theta, x_{1: T} \mid y_{1: T}\right)$ using the marginal $\mathrm{MH}$ algorithm. We can impose prior information through the prior density $p(\theta)$. The ideal marginal likelihood $p\left(y_{1: T} \mid \theta\right)$ is obtained from integrating out state variables $x_{1: T}$ :

$$
p\left(y_{1: T} \mid \theta\right)=\prod_{k=1}^{T} p\left(y_{k} \mid y_{1: k-1}, \theta\right)=\prod_{k=1}^{T} \int p\left(y_{k} \mid x_{k}, \theta\right) p\left(x_{k} \mid y_{1: k-1}, \theta\right) d x_{k} .
$$

Based on the above expression, the ideal acceptance ratio can be re-expressed as follows: 


$$
r\left(\theta^{*} \mid \theta\right)=\frac{p\left(y_{1: T} \mid \theta^{*}\right) p\left(\theta^{*}\right) q\left(\theta \mid \theta^{*}\right)}{p\left(y_{1: T} \mid \theta\right) p(\theta) q\left(\theta^{*} \mid \theta\right)}=\frac{\prod_{k=1}^{T} \int p\left(y_{k} \mid x_{k}^{*}, \theta^{*}\right) p\left(x_{k}^{*} \mid y_{1: k-1}, \theta^{*}\right) d x_{k} p\left(\theta^{*}\right) q\left(\theta \mid \theta^{*}\right)}{\prod_{k=1}^{T} \int p\left(y_{k} \mid x_{k}, \theta\right) p\left(x_{k} \mid y_{1: k-1}, \theta\right) d x_{k} p(\theta) q\left(\theta^{*} \mid \theta\right)}
$$

It is well known that, under the regularity conditions with the above ideal acceptance ratio, the realizations of the ideal marginal $\mathrm{MH}$ targeting $p\left(\theta \mid y_{1: T}\right)$ converge to its unique invariant distribution $\pi(A)=\int_{A} p\left(\theta^{*} \mid y_{1: T}\right) v\left(d \theta^{*}\right)$ with Lebesgue measure $v$ and $v$-measureable set $A \in \sigma(X)$ (Tierney, 1994). $\sigma(X)$ is the Borel sigma algebra of sets on $X \subseteq \mathbf{R}^{n}$.

In a linear state space model with Gaussian distribution assumption, we can calculate the exact form of $p\left(y_{k} \mid y_{1: k-1}, \theta\right)$ by using Kalman filter. However, we cannot obtain the analytical form of $p\left(y_{k} \mid y_{1: k-1}, \theta\right)$ in a nonlinear case so that we have to use an approximation method. In this study, we proposed two Gaussian-based filters, the CDKF and the GMF, to evaluate the pseudo-marginal likelihood $\hat{p}\left(y_{k} \mid y_{1: k-1}, \theta\right)$ in the PM-MH algorithm with the following acceptance ratio,

$$
\hat{r}^{k}\left(\theta^{*} \mid \theta\right)=\frac{\hat{p}^{k}\left(y_{1: T} \mid \theta^{*}\right) p\left(\theta^{*}\right) q\left(\theta \mid \theta^{*}\right)}{\hat{p}^{k}\left(y_{1: T} \mid \theta\right) p(\theta) q\left(\theta^{*} \mid \theta\right)} \text { for } k=\mathrm{CDKF}, \mathrm{GMF}
$$

Since the Gaussian-based filter deterministically evaluates the marginal likelihood without noises, minimizing approximation errors of the approximate likelihood is the key for parameter estimation when it is used in the MCMC algorithm. We note that if the acceptance ratio (1.35) converges to the true acceptance ratio (1.34), it guarantees that the PM-MH algorithm allows sampling from the true target density $p\left(\theta \mid y_{1: T}\right)$. This result could be achieved when the pseudo-marginal likelihood $\hat{p}^{k}\left(y_{1: T} \mid \theta^{*}\right)$ weakly converges to the true marginal likelihood $p\left(y_{1: T} \mid \theta^{*}\right)$.

In this section, we will establish that the PM-MH updates with the Gaussianbased filter leave the pseudo-posterior $\hat{p}^{k}\left(\theta \mid y_{1: T}\right)$ invariant for $k \in\{\mathrm{CDKF}, \mathrm{GMF}\}$ and that, under the standard assumptions, this sampler is ergodic. Although we 
focus on the CDKF and the GMF with PM-MH, these arguments can be generally applied to any Gaussian-based filters, including the UKF and the CKF. In addition, we will show that, under the GMF, the acceptance ratio (1.35) converges to (1.34) so that the realizations of the $\mathrm{MH}$ algorithm converges to having the true stationary density $p\left(\theta \mid y_{1: T}\right)$ when the number of Gaussian mixture components of the marginal likelihood goes to infinity. Since the PF within MCMC is widely discussed in Andrieu and Roberts (2009) and Andrieu et al. (2010), we focus on discussing the pseudomarginal MH updates with the Gaussian-based filters. Most of the arguments in this subsection are based on Tierney (1994), Roberts and Smith (1994), Mengersen and Tweedie (1996), Roberts and Tweedie (1996), Jarner and Hansen (2000), Geweke (2005), and Meyn and Tweedie (2012). Since Bayesian approaches to estimating DSGE models mostly rely on the random-walk-based Metropolis algorithm, we focus on the convergence properties of the random-walk-based Metropolis algorithm.

We define the supports of the pseudo-posterior $\hat{p}^{k}\left(\theta \mid y_{1: T}\right)$ and the proposal $q\left(\theta^{*} \mid \theta\right)$ as:

$$
\begin{gathered}
\left.\hat{\Theta}^{k}=\left\{\theta \in X: \hat{p}^{k}\left(\theta \mid y_{1: T}\right)>0\right)\right\}, \\
\hat{\Gamma}^{k}=\left\{\theta^{*} \in X: q\left(\theta^{*} \mid \theta\right)>0, \forall \theta \in \hat{\Theta}^{k}\right\},
\end{gathered}
$$

where $X \subseteq \mathbf{R}^{n}$ and $k \in\{\mathrm{CDKF}, \mathrm{GMF}\}$. The transition density of the PM-MH algorithm with the $k$ th Gaussian-based filter targets $\hat{p}^{k}\left(\theta \mid y_{1: T}\right)$ with a proposal density, $q\left(\theta^{*} \mid \theta\right)$ is defined as follows:

$$
\begin{aligned}
\hat{K}^{k}\left(\theta_{i}=\theta_{i}^{*} \mid \theta_{i-1}\right) & =\hat{\alpha}^{k}\left(\theta_{i}^{*} \mid \theta_{i-1}\right) q\left(\theta_{i}^{*} \mid \theta_{i-1}\right) I_{X_{\theta_{i-1}}}\left(\theta_{i}^{*}\right) \\
& +\delta\left(\theta_{i}^{*}-\theta_{i-1}\right)\left[1-\int_{X_{\theta_{i-1}}} \hat{\alpha}^{k}\left(\theta^{*} \mid \theta_{i-1}\right) q\left(\theta^{*} \mid \theta_{i-1}\right) d v\left(\theta^{*}\right)\right]
\end{aligned}
$$

where $X_{\theta_{i-1}}=\left\{\theta^{*} \in X: \theta^{*} \neq \theta_{i-1}\right\}$ and $\hat{\alpha}^{k}\left(\theta^{*} \mid \theta_{i-1}\right)=\min \left[1, \hat{r}^{k}\left(\theta^{*} \mid \theta_{i-1}\right)\right]$. The first term is the probability of accepting the proposed $\theta_{i}^{*}$ and the second term is the 
probability of rejecting the proposed $\theta_{i}^{*}$. The transition probability measure of the PM-MH is defined as $\hat{\mathbf{P}}^{k}(A \mid \theta)=\int_{A} \hat{K}^{k}\left(\theta^{*} \mid \theta\right) v\left(d \theta^{*}\right)$ with $v$-measurable sets $A \in \sigma(X)$.

We define the pseudo-invariant distribution $\hat{\pi}^{k}(A)$ as

$$
\hat{\pi}^{k}(A)=\int_{A} \hat{p}^{k}\left(\theta^{*} \mid y_{1: t}\right) v\left(d \theta^{*}\right)
$$

and define the distribution of $i$ th iteration of the chain conditional on the initial $\theta_{0}$ as

$$
\hat{\mathbf{P}}^{k, i}\left(A \mid \theta_{0}\right)=\int \hat{\mathbf{P}}^{k, i-1}\left(A \mid \theta^{*}\right) \hat{\mathbf{P}}^{k}\left(d \theta^{*} \mid \theta_{0}\right) .
$$

Assumption 1. Ergodicity and Aperiodicity: The PM-MH sampler of target pseudo-posterior $\hat{p}^{k}\left(\theta \mid y_{1: T}\right)$ and proposal $q\left(\theta^{*} \mid \theta\right)$ is $\hat{\pi}$-irreducible and aperiodic.

Assumption 2. Geometric drift towards a small set C: There exists a drift function $V \geq 1$, finite for at least one $\theta$, and constants $\lambda<1$ and $b<\infty$ such that

$$
\hat{\mathbf{P}}^{k} V(\theta) \leq \lambda V(\theta)+b I_{C}(\theta)
$$

where $\hat{\mathbf{P}}^{k} V(\theta)=\int V\left(\theta^{*}\right) \hat{\mathbf{P}}^{k}\left(d \theta^{*} \mid \theta\right)$ and $I_{C}(\theta)$ is the indicator function of a small set C. A set $C$ is called small if there exist $n>0, \delta>0$, and a probability measure $Q$ such that

$$
\hat{\mathbf{P}}^{k, n}(A \mid \theta) \geq \delta Q(A) \quad \forall \theta \in C, A \in \sigma(X),
$$

The first assumption ensures that the PM-MH algorithm with the Gaussianbased filter has the unique invariant distribution $\hat{\pi}^{k}(A)$ satisfying

$$
\left\|\hat{\mathbf{P}}^{k, i}\left(A \mid \theta_{0}\right)-\hat{\pi}^{k}(A)\right\| \rightarrow 0 \quad \text { for any } \theta_{0} \in \hat{\Theta}^{k} \text { as } i \rightarrow \infty
$$

where $\|\cdot\|$ denotes the total variation distance. The existence of the unique pseudoinvariant distribution and the convergence of the chain are direct consequences of The- 
orem 1 of Tierney (1994). Sufficient conditions for the first assumption (irreducibility and aperiodicity) of the random-walk-based Metropolis Hastings algorithm have previously been studied by Tierney (1994), Roberts and Smith (1994), Mengersen and Tweedie (1996), Roberts and Tweedie (1996), Jarner and Hansen (2000), and Geweke (2005). For instance, if the support of the proposal $q\left(\theta^{*} \mid \theta\right)$ includes the support of the pseudo-posterior $\hat{p}^{k}\left(\theta \mid y_{1: T}\right)\left(\hat{\Theta}^{k} \subseteq \hat{\Gamma}^{k}\right)$, then the PM-MH algorithm is ergodic (see Theorem 4.5.5 of Geweke (2005)). Since the support of the Normal proposal density for the random-walk-based Metropolis is $\mathbf{R}^{n}$, the PM-MH algorithm for the Monte Carlo study in Section 5 satisfies the sufficient condition for convergence.

With these convergence properties, we discuss conditions that lead the PMMH to the true posterior for both the CDKF and the GMF. The following argument discusses conditions where the PM-MH with the CDKF converge to the true posterior distribution.

Remark 1. Although the CDKF does not always approximate the true marginal likelihood, and hence the true posterior distribution, especially in a nonlinear and nonGaussian model, the estimated first and second moments through the CDKF are accurate up to at least the 2nd order and are accurate up to the 3rd order when the state vector is approximately Gaussian (Andreasen, 2013). This implies that if the following two conditions are satisfied: (1) the true marginal likelihood is well approximated by a Gaussian distribution; (2) the higher order terms beyond third order are insignificant, the estimated first moment and second moment of the pseudo-posterior distribution $\hat{p}^{C D K F}\left(\theta \mid y_{1: T}\right)$ calculated by the CDKF with PM-MH could be consistent with the first and second moments of the true posterior distribution $p\left(\theta \mid y_{1: T}\right){ }^{6}$

If the true marginal likelihood is far from Gaussian, the GMF can be used to

${ }^{6}$ The CDKF might not satisfy these sufficient conditions, depending on how nonlinear the model is. According to the Monte Carlo exercises in Section 5, the CDKF seems to satisfy the conditions in the benchmark and nearly linear cases (except for a few cases in the benchmark case), but does not satisfy the conditions when the model is highly nonlinear. 
complement the CDKF. For ease of exposition of the GMF with PM-MH, we make the following assumption.

Assumption 3. The predicted and filtered densities obtained from the Gaussian mixture filter weakly converges to the true predicted and filtered densities as the number of mixture components goes to infinity.

Under Assumption 3, we can derive the following convergence property of the GMF with the PM-MH sampler.

Corollary 1. Under Assumption 1 and Assumption 3, the pseudo-marginal MH with Gaussian mixture filter generates a sequence $\left\{\theta^{(i)}\right\}$ whose marginal distributions $\hat{P}_{i}^{G M F}\left(A \mid \theta_{0}\right)$ satisfy

$$
\left.\| \hat{\mathbf{P}}^{G M F, i}\left(A \mid \theta_{0}\right)-\pi(A)\right) \| \rightarrow 0 \quad \text { for any } \theta_{0} \in \hat{\Theta}^{k} \text { as } J \rightarrow \infty \text { and } i \rightarrow \infty
$$

where $J$ is the total number of mixture components of the marginal likelihood. The statement builds on the fact that if the total number of mixture components of the marginal likelihood goes to infinity, the Gaussian mixture marginal likelihood weakly converges to the true marginal likelihood, and hence the acceptance ratio of the PM-MH algorithm converges to that of the ideal marginal MH algorithm.

Assumption 2 is required for the geometric convergence of the PM-MH algorithm. If the pseudo-marginal MH with Gaussian-based filter is $\hat{\pi}$-irreducible and aperiodic, and Assumption 2 holds, then the chain is geometrically ergodic.

Corollary 2. If the pseudo-marginal MH with Gaussian mixture filter is $\hat{\pi}$-irreducible and aperiodic (Assumption 1), and Assumption 2 holds,

$$
\left.\| \hat{\mathbf{P}}^{G M F, i}\left(A \mid \theta_{0}\right)-\pi(A)\right) \| \leq R V\left(\theta_{0}\right) \rho^{i} \text { for any } \theta_{0} \in \Theta, \text { as } J \rightarrow \infty
$$

where $V(\cdot)$ denotes a drift function, satisfying (1.41), $\rho<1$, and $R<\infty$. J 
is the total number of mixture components of the marginal likelihood. $\Theta$ is the support set of the true posterior $p\left(\theta \mid y_{1: T}\right)$. The statement is a direct consequence of the convergence of the Gaussian mixture approximation. Although Mengersen and Tweedie (1996), Roberts and Tweedie (1996), and Jarner and Hansen (2000) provide sufficient conditions for the geometrically ergodic random-walk-based Metropolis, it is not feasible to verify the conditions for complicated and high-dimensional DSGE models (Roberts and Rosenthal, 1998). ${ }^{7}$ The details of proofs for each argument are given in Appendix A.3.

\subsubsection{Asymptotic Posterior Normality}

In this section, we discuss the asymptotic properties of pseudo-posterior distributions based on the pseudo-marginal likelihood implied by Gaussian-based filters when the full information on the true data generating process (the true likelihood) is possibly misspecified. Theorem 2 of Müller (2013) can be directly applicable in our case. We define the pseudo log-likelihood of $y_{1: T}$ by $\hat{L}_{T}^{k}(\theta)=\sum_{t=1}^{T} \hat{l}_{t}^{k}(\theta)$ where $\hat{l}_{t}^{k}(\theta)=$ $\ln \left(\hat{p}^{k}\left(y_{t} \mid y_{1: t-1} ; \theta\right)\right)$ and $k \in\{\mathrm{CDKF}, \mathrm{GMF}\}$. We define the score as $\hat{s}_{t}^{k}(\theta)=\partial \hat{l}_{t}^{k}(\theta) / \partial \theta$ and the Hessian as $\hat{h}_{t}^{k}(\theta)=\partial \hat{s}_{t}^{k}(\theta) / \partial \theta^{\prime}{ }^{8}$ Let $\left\{y_{t}\right\}$ be a sequence of dependent timeseries observable variables. Under regularity conditions, such as (1) stationary and ergodic $\left\{y_{t}\right\}$ and (2) a martingale difference sequence of the scores $\left\{\hat{s}_{t}^{k}(\theta)\right\}$ with respect to $\left\{y_{t}\right\}$, we can apply a law of large numbers for consistency of pseudo maximum likelihood estimators (PMLE) $\hat{\theta}^{k}$ for the pseudo-true values $\theta_{0}^{k}$ that minimize the Kullback-Leibler distance between the true likelihood and the pseudo likelihood,

\footnotetext{
${ }^{7}$ Mengersen and Tweedie (1996) and Roberts and Tweedie (1996) verify that a random-walkbased Metropolis is geometrically ergodic when the target invariant distribution has exponentially decreasing tails (in one dimension) and behaves sufficiently smoothly in the tails (in higher dimension). Jarner and Hansen (2000) shows more general conditions for geometric ergodicity. Although a random-walk-based $\mathrm{MH}$ sampler might fail to satisfy these conditions, it can be polynomially ergodic of all orders (Fort and Moulines, 2000).

${ }^{8}$ We calculate the scores $\hat{s}_{t}^{k}(\theta)$ and the Hessians $\hat{h}_{t}^{k}(\theta)$ by numerically differentiating the conditional likelihoods $\hat{l}_{t}^{k}(\theta)$
} 
and can apply a central limit theorem for asymptotic normality of $\hat{\theta}^{k}$ (White, 1982; Bollerslev and Wooldridge, 1992; Müller, 2013; Hall and Heyde, 2014). Specifically,

$$
\sqrt{T}\left(\hat{\theta}^{k}-\theta_{0}^{k}\right) \stackrel{\mathrm{d}}{\rightarrow} N\left(0, \Omega_{S}^{k}\left(\theta_{0}^{k}\right)\right)
$$

where $\Omega_{S}^{k}\left(\theta_{0}^{k}\right)=\Omega_{M}^{k}\left(\theta_{0}^{k}\right) V^{k}\left(\theta_{0}^{k}\right) \Omega_{M}^{k}\left(\theta_{0}^{k}\right), \Omega_{M}^{k}\left(\theta_{0}^{k}\right)=-E\left[\frac{\partial^{2} \hat{L}_{T}^{k}(\theta)}{\partial \theta \partial \theta^{\prime}} ; \theta=\theta_{0}^{k}\right]^{-1}$, and $V^{k}\left(\theta_{0}^{k}\right)=$ $E\left[\hat{s}_{t}^{k}\left(\theta_{0}^{k}\right) \hat{s}_{t}^{k^{\prime}}\left(\theta_{0}^{k}\right)\right]$

Given the pseudo log-likelihood $\hat{L}_{T}^{k}(\theta)$ and prior density $p(\theta)$, the pseudo-posterior density $\hat{p}^{k}\left(\theta \mid y_{1: T}\right)$ is defined as

$$
\hat{p}^{k}\left(\theta \mid y_{1: T}\right)=\frac{\exp \left(\hat{L}_{T}^{k}(\theta)\right) p(\theta)}{\int_{\hat{\Theta}^{k}} \exp \left(\hat{L}_{T}^{k}(\theta)\right) p(\theta) d \theta} .
$$

Under regularity assumptions outlined in Müller (2013), we can derive the following Corollary based on Theorem 2 of Müller (2013).

Corollary 3. (a) Given the pseudo-marginal likelihood implied by Gaussian-based filters,

$$
\left\|\hat{\pi}^{k}-N\left(\hat{\theta}^{k}, \Omega_{M}^{k}\left(\theta_{0}^{k}\right) / n\right)\right\| \stackrel{p}{\rightarrow} 0
$$

where $\|\cdot\|$ denotes the total variation distance, $\hat{\pi}^{k}$ is the pseudo-posterior distribution, $\hat{\theta}^{k}$ is equal to the PMLE, and $\Omega_{M}^{k}\left(\theta_{0}^{k}\right)=-E\left[\frac{\partial^{2} \hat{L}_{T}^{k}(\theta)}{\partial \theta \partial \theta^{\prime}} ; \theta=\theta_{0}^{k}\right]^{-1}$.

(b) Given the pseudo-marginal likelihood implied by the Gaussian mixture filter,

$$
\left\|\hat{\pi}^{G M F}-N\left(\theta_{0}, \Omega_{M}^{0}\left(\theta_{0}\right) / n\right)\right\| \stackrel{p}{\rightarrow} 0 \quad \text { as } J \rightarrow \infty
$$

where $J$ is the total number of mixture components of the marginal likelihood, $\hat{\pi}^{G M F}$ is the pseudo-posterior distribution implied by the Gaussian mixture filter, $\theta_{0}$ is equal to the true $M L E$, and $\Omega_{M}^{0}\left(\theta_{0}\right)=\Omega_{S}^{0}\left(\theta_{0}\right)$ under the true marginal likelihood.

The statement implies that the pseudo-posterior implied by the misspecified 
marginal likelihood converges to a Gaussian distribution whose mean and variance equal to the PMLE $\hat{\theta}^{k}$ and $\Omega_{M}^{k}\left(\theta_{0}^{k}\right) / n$, respectively. Under a case of misspecified likelihood, $\Omega_{M}^{k}\left(\theta_{0}^{k}\right) / n$ is not of the sandwich type so that the sandwich covariance matrix correction is required to lower the risk of Bayesian inference. Under (approximately) correct specification, inference based on the (approximately) exact posterior distribution provides asymptotically lower sample Bayes risk. In this case, it can be shown that $\Omega_{M}^{0}\left(\theta_{0}\right)=\Omega_{S}^{0}\left(\theta_{0}\right)$. Since the GMF and the PF inherently have this characteristic, we apply the sandwich covariance matrix correction to the CDKF with PM-MH in the following Monte Carlo study.

\subsection{Monte Carlo Study}

To evaluate the performance of the Gaussian-based filters, we first calculate the root mean squared errors (RMSEs) of the filtered state variables by using 'artificial' data generated from the model and compare the RMSEs of the Gaussian-based filters with those of the PF. Since the PF inherently calculate noisy filtered state variables, we report its RMSEs with a 95\% confidence interval based on 100 samples of filtered state variables. The 'artificial' data are simulated from the solution of the model for given calibrated parameter values. For given true parameter values, we compute the RMSEs as follows:

$$
R M S E_{k}=\sum_{i=1}^{N} \sqrt{\frac{\sum_{t=1}^{T}\left(x_{i, t}-\hat{x}_{i, t \mid t}\right)^{2}}{T}} \text { for } k=C D K F / G M F / P F
$$

where $x_{i, t}$ is the 'realized' value of the $i$ th state variable at time $t$ and $\hat{x}_{i, t \mid t}$ is the $i$ th filtered state variable. Then, we estimate the nonlinear neoclassical growth model with 'artificial' data by using the PM-MH and evaluate its performance based on the 
average relative biases (ARBs) of posterior means as follows:

$$
A R B s_{k}=\sqrt{\frac{1}{N} \sum_{i=1}^{N}\left(\frac{\left(\hat{\theta}_{i}-\theta_{i}^{\text {true }}\right)}{\theta_{i}^{\text {true }}}\right)^{2}} \text { for } k=C D K F / G M F / P F
$$

where $\theta_{i}^{\text {true }}$ is the $i$ th true parameter value and $\hat{\theta}_{i}$ is the $i$ th posterior mean. For the estimation, we impose priors on parameters and draw from their posterior distributions through the PM-MH algorithm implied by each filtering method; two Gaussian-based filters and the PF. Finally, we use the modified harmonic mean estimator for the marginal likelihood implied by each nonlinear approximation filter (Geweke, 1999),

$$
\begin{aligned}
P\left(Y \mid M_{k}\right) & =E\left[\frac{g\left(\theta \mid M_{k}\right)}{p\left(Y \mid \theta, M_{k}\right) p\left(\theta \mid M_{k}\right)} \mid Y\right]^{-1} \text { for } k=C D K F / G M F / P F \\
& =\left[\frac{1}{N} \sum_{i=1}^{N} \frac{g\left(\theta^{i} \mid M_{k}\right)}{p\left(Y \mid \theta^{i}, M_{k}\right) p\left(\theta^{i} \mid M_{k}\right)} \mid Y\right]^{-1},
\end{aligned}
$$

where $g\left(\theta \mid M_{k}\right)$ is a truncated multivariate normal distribution proposed by Geweke (1999b) and has the property that $\int g\left(\theta \mid M_{k}\right) d \theta=1 . \theta^{i}$ is the $i$ th sample drawn from the posterior distribution. We report the marginal likelihoods with three truncation parameters of $0.1,0.5$, and 0.9 .

This exercise answers the following three questions: (1) How accurate is the estimation of the true parameter values and the 'realized' state variables implied by each filter? (2) Are the Gaussian-based filters computationally efficient? (3) How well do the Gaussian-based filters with PM-MH explain data? We address the first question based on the RMSEs and the ARBs. To answer the second question, we calculate the effective computing time by dividing the computing time of MCMC by the multivariate effective sample size $N\left(\frac{\Lambda}{\Sigma}\right)^{1 / p}$ proposed by Vats et al. (2017) where $\Lambda$ is the asymptotic covariance matrix based on independent draws, $\Sigma$ is the asymptotic covariance matrix of correlated samples, $p$ is the number of parameters, 
and $N$ is the number of simulated samples. ${ }^{9}$ The MCMC sampler might have a different effective sample size, depending on which filter we use. For example, 50,000 samples from a Markov chain with a certain filter are worth about as much as 500 independent samples. On the other hand, 50,000 samples from a Markov chain with a different filter are worth about as much as 300 independent samples. Since we are interested in posterior means, the computing time of $\mathrm{MCMC}$ is not enough to evaluate the computational efficiency. Finally, we respond to the third question by using the marginal likelihood.

Since the dynamics of policy variables greatly depend on parameter values, we perform four different exercises with four different sets of parameter values, one with benchmark parameter values following Fernández-Villaverde and Rubio-Ramírez (2005), Fernández-Villaverde (2010), and Justiniano et al. (2010), an extremely nonlinear case with a high Frisch labor supply elasticity and a large standard deviation of the technology shock, and a nearly linear case with a low Frisch labor supply elasticity and a small standard deviation of the technology shock. All models are solved to the second order. Finally, we investigate the benchmark model with stochastic volatility solved up to third order. We summarize the calibrated parameter values in Table 1.2. The coefficients of the second-order approximation for given calibrated parameters are given in Appendix A.4.

\subsubsection{The Priors}

We assume diffuse priors for all 11 parameters in the benchmark case and 12 parameters in the benchmark with stochastic volatility, giving some restrictions on parameter regions. Since we are going to use 100 observations in the exercises, the estimation results might be affected by the prior elicitation. To avoid the artificially estimated

\footnotetext{
${ }^{9}$ The effective sample size is used to assess the convergence of sums of MCMC samples in a heuristic way.
} 
results, we do not incorporate any prior information for the estimation so that we make the estimation results fully come from the information of the likelihood function itself. In this sense, our exercise is closely related to the maximum likelihood estimate. We can achieve this goal by allowing uniform prior distributions.

The persistence of the technology shock and investment-specific technology shock follow a uniform distribution between 0 and 1. The parameter $\sigma$ which controls the Frisch labor supply elasticity follows a uniform distribution between 0 and 100 . The prior for the labor share, $\alpha$, is a uniform between 0 and 1 . The prior on the depreciation rate follows a uniform distribution between 0 and 0.05 . The discount factor, $\beta$, is allowed to follow uniform with the interval of 0.75 and 1 . The standard deviations of both technology and investment-specific technology shock follow a uniform distribution between 0 and 100. We also pick the uniform distribution for the standard deviations of the measurement errors $\left(\sigma_{1 m}, \sigma_{2 m}, \sigma_{3 m}\right)$. The persistence $\rho_{\sigma_{z}}$ and standard deviation $\sigma_{\sigma_{z}}$ in the stochastic volatility process follow uniform over $[0,1]$ and uniform over $[0,100]$, respectively. Table 1.2 summarizes our discussion.

Table 1.2 Calibrated Parameters and Priors

\begin{tabular}{c|c|c|c|c|c}
\hline & Benchmark & $\begin{array}{c}\text { Extremely } \\
\text { Nonlinear }\end{array}$ & $\begin{array}{c}\text { Nearly } \\
\text { Linear }\end{array}$ & $\begin{array}{c}\text { Benchmark } \\
\text { with Vol }\end{array}$ & Prior \\
\hline$\alpha$ & 0.67 & 0.67 & 0.67 & 0.67 & Uniform[0.3, 1] \\
$\beta$ & 0.99 & 0.99 & 0.99 & 0.99 & Uniform[0.75, 1] \\
$\delta$ & 0.025 & 0.025 & 0.025 & 0.025 & Uniform[0, 0.05] \\
$\sigma$ & 2.75 & 0.02 & 50 & 2.75 & Uniform[0, 100] \\
$\rho_{z}$ & 0.95 & 0.95 & 0.95 & 0.95 & Uniform[0, 1] \\
$\rho_{\mu}$ & 0.72 & 0.72 & 0.72 & 0.72 & Uniform[0, 1] \\
$\sigma_{z}$ & 0.007 & 0.035 & 0.007 & - & Uniform[0, 100] \\
$\sigma_{\mu}$ & 0.06 & 0.06 & 0.007 & 0.06 & Uniform[0, 100] \\
$\sigma_{1 m}$ & 0.004 & 0.004 & 0.004 & 0.004 & Uniform[0, 100] \\
$\sigma_{2 m}$ & 0.019 & 0.019 & 0.019 & 0.019 & Uniform[0, 100] \\
$\sigma_{3 m}$ & 0.009 & 0.009 & 0.009 & 0.009 & Uniform[0, 100] \\
$\rho_{\sigma_{z}}$ & - & - & - & 0.76 & Uniform[0, 1] \\
$\sigma_{\sigma_{z}}$ & - & - & - & 0.392 & Uniform[0, 100] \\
\hline
\end{tabular}




\subsubsection{Results with 'Artificial' Data}

\section{Benchmark Case}

We simulate 'artificial' data for consumption, investment, and hours worked from the model and use them for the estimation. As a benchmark case of the model, we calibrate parameter values based on actual data and previous studies to make benchmark experiment as realistic as possible. The average Fed Funds rate (1.4\%) at a quarterly frequency implies that the discount factor $\beta$ is 0.99 . The value of $\sigma$ is chosen to set the Frisch labor supply elasticity to nearly 0.85 obtained by Fernández-Villaverde (2010). We set the labor share of national income $\alpha$ to 0.67 . The depreciation rate $\delta$ is set to 0.025. As described in Fernández-Villaverde and Rubio-Ramírez (2005), $\rho_{z}=0.95$ and $\sigma_{z}=0.007$ match the Solow residual of the US economy. The parameters of the investment-specific technology shock process are $\rho_{\mu}=0.72$ and $\sigma_{\mu}=0.06$, both of

which are based on those obtained from Justiniano et al. (2010). With respect to the standard deviations of measurement errors, we set them equal to 0.004 for consumption, 0.019 for investment, and 0.009 for hours worked. These values are calculated by the half of the standard deviations of hp-filtered actual data.

Using the simulated data for 100 periods, we apply our priors and likelihood evaluation algorithms. As one of the SPKF, we use the CDKF. For the GMF with Binomial Gaussian mixture, we use two Gaussian-mixture components for the initial filtered distribution, set the threshold $\eta_{\text {limit }}$ to 0.25 , and set threshold $B_{\text {limit }}$ to 0.001 . The thresholds, $m_{\text {limit }}$ and $m_{\text {reduce }}$ for splitting and reduction procedures are set to 32 and 8, respectively. For the PF, we use 500 particles. We run the PM-MH algorithm over 55,000 iterations, discard the first 5,000 as burn-in, and finally get 50,000 draws from the posterior distribution. All computations are done in Matlab on Intel(R) Core(TM) i5-2500 CPU 3.3GHz, 8 GB memory.

Table 1.3 shows that the GMF gives marginally more accurate filtered state 
Figure 1.1: Posterior Distribution: Benchmark Case
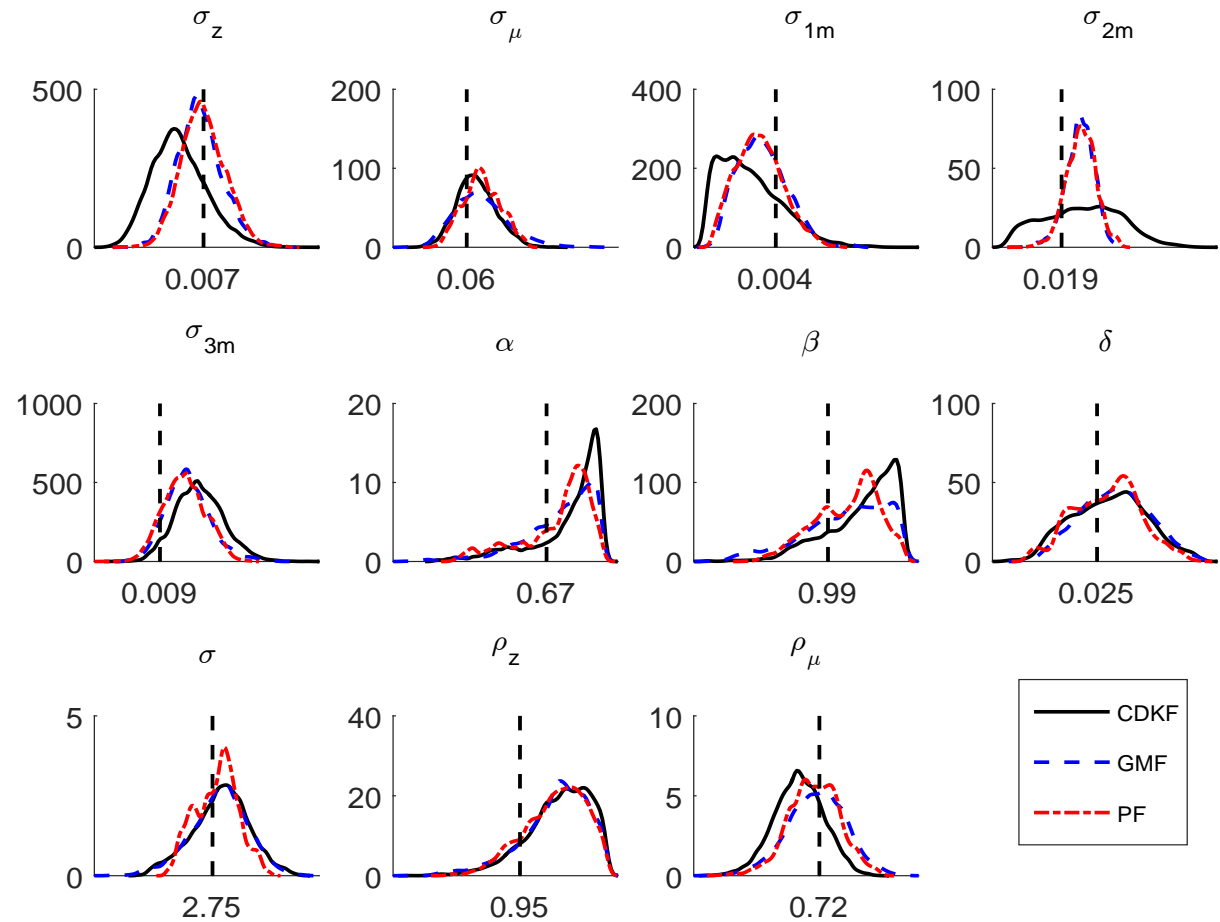

Notes. The vertical dashed line represents true parameter values.

Table 1.3 Posterior Distribution: Benchmark Case

\begin{tabular}{c|ccc|ccc|ccc}
\hline & \multicolumn{4}{|c|}{ CDKF } & \multicolumn{4}{c|}{ GMF } & \multicolumn{4}{c}{ PF } \\
\hline & Mean & S.D. & Prob. Interval & Mean & S.D. & Prob. Interval & Mean & S.D. & Prob. Interval \\
\hline$\alpha$ & 0.706 & 0.011 & $(0.686,0.725)$ & 0.692 & 0.058 & $(0.579,0.755)$ & 0.688 & 0.057 & $(0.563,0.751)$ \\
$\beta$ & 0.994 & 0.001 & $(0.991,0.996)$ & 0.992 & 0.006 & $(0.981,0.999)$ & 0.992 & 0.004 & $(0.985,0.999)$ \\
$\delta$ & 0.028 & 0.001 & $(0.026,0.030)$ & 0.029 & 0.008 & $(0.016,0.042)$ & 0.027 & 0.008 & $(0.015,0.040)$ \\
$\sigma$ & 2.795 & 0.114 & $(2.602,2.987)$ & 2.787 & 0.155 & $(2.515,3.029)$ & 2.773 & 0.114 & $(2.579,2.963)$ \\
$\rho_{z}$ & 0.972 & 0.021 & $(0.937,0.999)$ & 0.97 & 0.02 & $(0.932,0.996)$ & 0.97 & 0.018 & $(0.938,0.995)$ \\
$\rho_{\mu}$ & 0.668 & 0.083 & $(0.529,0.807)$ & 0.713 & 0.075 & $(0.585,0.831)$ & 0.707 & 0.065 & $(0.599,0.814)$ \\
$\sigma_{z}$ & 0.006 & 0.001 & $(0.003,0.008)$ & 0.007 & 0.001 & $(0.005,0.008)$ & 0.007 & 0.001 & $(0.006,0.009)$ \\
$\sigma_{\mu}$ & 0.062 & 0.005 & $(0.053,0.070)$ & 0.063 & 0.006 & $(0.054,0.074)$ & 0.063 & 0.004 & $(0.057,0.071)$ \\
$\sigma_{1 m}$ & 0.003 & 0.002 & $(0.000,0.006)$ & 0.003 & 0.001 & $(0.001,0.006)$ & 0.003 & 0.001 & $(0.001,0.005)$ \\
$\sigma_{2 m}$ & 0.026 & 0.028 & $(0.000,0.072)$ & 0.026 & 0.005 & $(0.017,0.033)$ & 0.026 & 0.005 & $(0.017,0.034)$ \\
$\sigma_{3 m}$ & 0.01 & 0.001 & $(0.009,0.011)$ & 0.01 & 0.001 & $(0.009,0.011)$ & 0.01 & 0.001 & $(0.009,0.011)$ \\
\hline
\end{tabular}

Effective Computing Time

\begin{tabular}{|c|c|c|c|}
\hline & 0.002 & 0.019 & 0.685 \\
\hline \multicolumn{4}{|c|}{ Root Mean Squared Errors (RMSEs) } \\
\hline & 0.0138 & 0.0098 & $(0.0092,0.0123)$ \\
\hline \multicolumn{4}{|c|}{ Average Relative Biases (ARBs) } \\
\hline & 0.3504 & 0.2783 & 0.2961 \\
\hline
\end{tabular}


Table 1.4 Logmarginal Likelihood: Benchmark Case

\begin{tabular}{c|ccc}
\hline $\mathrm{p}$ & $\mathrm{CDKF}$ & $\mathrm{GMF}$ & $\mathrm{PF}$ \\
\hline 0.1 & 527.62 & 572.43 & 572.07 \\
0.5 & 527.79 & 572.96 & 572.39 \\
0.9 & 527.90 & 572.96 & 571.87 \\
\hline
\end{tabular}

variables than the CDKF, and the RMSEs of the GMF is inside the $95 \%$ confidence interval of the RMSEs from the PF. We also note that the RMSEs for the CDKF are marginally larger than the RMSEs confidence interval for the PF.

Figure 1.1 reveals that most of the posterior distributions of parameters have the similar shapes and are centred on the true values, though the CDKF delivers a slightly different position and dispersion, especially for the standard deviation of technology shock, $\sigma_{z}$, the standard deviations of measurement errors, $\sigma_{2 m}$ and $\sigma_{3 m}$, and the persistence of investment-specific technology shock, $\rho_{\mu}$. Since this exercise is using diffuse priors, none of the posterior distributions are driven by the priors. The asymmetry or non-Gaussianity of the pseudo-posterior distributions implied by the CDKF indicates larger asymptotic variances of $\sigma_{2 m}, \sigma_{3 m}$, and $\rho_{\mu}$ than those of the GMF and the PF, requiring large sample size to have a Gaussian form. Table 1.3 conveys similar information: the point estimates are close to the true parameter values regardless of the filters, the true values are inside the $90 \%$ posterior probability intervals in most of the cases, and the asymptotic sandwich variances of $\sigma_{2 m}, \sigma_{3 m}$, and $\rho_{\mu}$ for the CDKF are larger than those obtained by the GMF and the PF. Moreover, the GMF delivers estimates that are better in the sense of being closer to the true values. The ARBs for the GMF are the smallest among the three estimation procedures. The ARBs for the CDKF are marginally smaller than those for the PF.

The performance of the Gaussian-based filters can also be evaluated by their effective computing time. Table 1.3 reports the effective computing time for each filtering method with PM-MH. The CDKF and the GMF are much more efficient 
than the PF to run PM-MH estimation. The value obtained from the CDKF with PM-MH is 0.002, and the GMF with PM-MH takes 0.019. However, the PF with PM-MH takes 0.685 even though we use 500 particles for the estimation. Considering the small differences in the estimated posterior distributions, the Gaussian-based filters with PM-MH are more attractive for the evaluation of likelihood and posterior inference than the PF with PM-MH in this benchmark exercise.

Table 1.4 reports the logmarginal likelihood between the Gaussian-based filters with PM-MH and the PF with PM-MH. We compute the marginal likelihood with Gewekes (1998) harmonic mean proposal. It shows that the logmarginal likelihood of the GMF with PM-MH is higher than other filters. However, the CDKF with PM-MH delivers a lower logmarginal likelihood than those of the GMF with PM-MH and the PF with PM-MH.

\section{Extremely Nonlinear Case}

We implement the same exercise with extreme values of parameters, allowing highly nonlinear dynamics of the model. The second calibration maintains the same parameter values as those in the benchmark case, except that it decreases $\sigma$ to 0.02 and increases $\sigma_{z}$ to 0.035 . The low value of $\sigma$ allows nearly infinite Frisch labor elasticity implying that the model is observationally equivalent to the indivisible labor models of Hansen (1985) and Rogerson (1988). Although it differs from the common estimates which are the neighborhood of unity, it is supported by Sims (2011) and can generate high volatility of hours worked. The high Frisch labor supply elasticity and high standard deviation of technology shock introduce a strong nonlinearity to the economy. Although the extreme values of parameters might not be realistic, this

particular choice of parameter values allows us to check the differences between the Gaussian-based filters with PM-MH and the PF with PM-MH in a highly nonlinear world. 
Table 1.5 shows that the GMF gives marginally more accurate filtered state variables than the CDKF. The RMSEs of the CDKF and the GMF are both inside the $95 \%$ confidence interval of the RMSEs from the PF. Figure 1.2 represents posterior distributions for the extremely nonlinear case. In this extreme case, the GMF with PM-MH and the PF with PM-MH show good performance, but the CDKF with PM-MH generates rather poor performance than the benchmark case. Especially, the CDKF creates upward biases for the labor share $\alpha$, the discount factor $\beta$, and the parameter $\sigma$ that controls the labor supply elasticity. In addition, the posterior distributions implied by the CDKF are more dispersed than those implied by the GMF and PF. The poorly identified parameters in the extremely nonlinear world may cause the biased parameters and large posterior probability intervals (Canova and Sala, 2009).

Table 1.5 delivers the same information as that of Figure 1.2, in terms of posterior means and asymptotic standard deviations. The posterior probability intervals calculated by the CDKF with PM-MH do not capture the true values of $\alpha$ and $\sigma$. Moreover, the pseudo-posterior standard deviations are larger than those of the GMF with PM-MH and the PF with PM-MH. The ARBs in the extremely nonlinear case are larger than those in the benchmark case. The ARBs for the CDKF are nearly twenty times larger than those of the benchmark case. Although the GMF and the PF have rather large values of ARBs, they could be reduced by allowing more mixture components and particles with large samples. Overall, the GMF with PM-MH and the PF with PM-MH are better than the CDKF with PM-MH in the extremely nonlinear case. Among the three estimation procedures, the GMF shows the best performance with the smallest ARBs. The effective computing time of the CDKF with PM-MH is still much smaller than that of the GMF with PM-MH and PF with PM-MH. However, the effective computing time of the GMF is increased dramatically compared to that of benchmark case because the splitting procedure of the GMF 
Figure 1.2: Posterior Distribution: Extremely Nonlinear Case
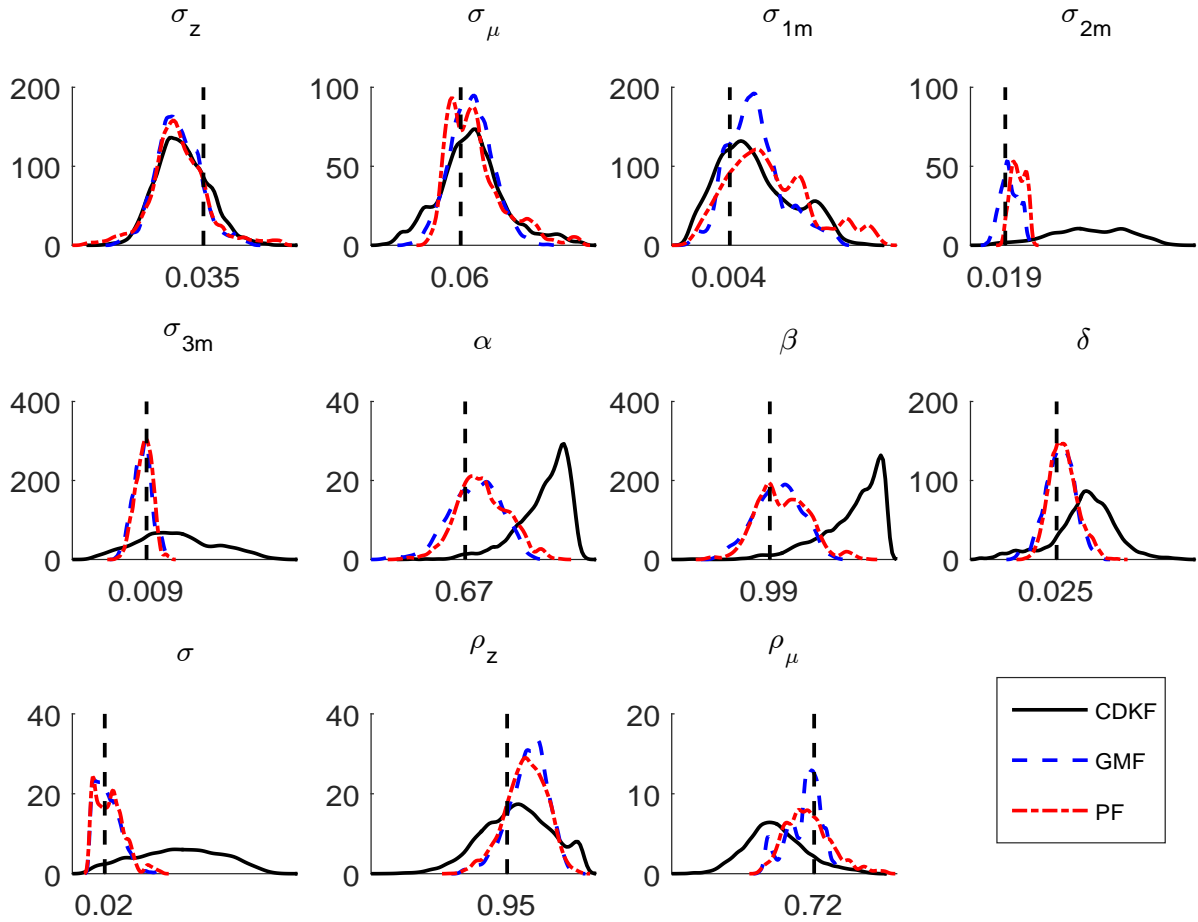

Notes. The vertical dashed line represents true parameter values.

Table 1.5 Posterior Distribution: Extremely Nonlinear Case

\begin{tabular}{c|ccc|ccc|ccc}
\hline & \multicolumn{4}{|c|}{ CDKF } & \multicolumn{4}{c|}{ GMF } & \multicolumn{4}{c}{ PF } \\
\hline & Mean & S.D. & Prob. Interval & Mean & S.D. & Prob. Interval & Mean & S.D. & Prob. Interval \\
\hline$\alpha$ & 0.73 & 0.0329 & $(0.674,0.785)$ & 0.677 & 0.02 & $(0.645,0.708)$ & 0.683 & 0.02 & $(0.653,0.716)$ \\
$\beta$ & 0.997 & 0.0042 & $(0.989,0.999)$ & 0.991 & 0.002 & $(0.987,0.994)$ & 0.991 & 0.002 & $(0.988,0.995)$ \\
$\delta$ & 0.03 & 0.0081 & $(0.016,0.044)$ & 0.026 & 0.003 & $(0.021,0.030)$ & 0.026 & 0.003 & $(0.022,0.031)$ \\
$\sigma$ & 0.119 & 0.0131 & $(0.096,0.141)$ & 0.024 & 0.017 & $(0.002,0.055)$ & 0.027 & 0.019 & $(0.002,0.060)$ \\
$\rho_{z}$ & 0.957 & 0.0244 & $(0.916,0.998)$ & 0.964 & 0.013 & $(0.940,0.983)$ & 0.962 & 0.014 & $(0.938,0.983)$ \\
$\rho_{\mu}$ & 0.634 & 0.147 & $(0.386,0.881)$ & 0.696 & 0.039 & $(0.617,0.749)$ & 0.707 & 0.052 & $(0.631,0.803)$ \\
$\sigma_{z}$ & 0.033 & 0.0027 & $(0.028,0.038)$ & 0.032 & 0.003 & $(0.028,0.036)$ & 0.032 & 0.003 & $(0.027,0.038)$ \\
$\sigma_{\mu}$ & 0.062 & 0.0103 & $(0.044,0.079)$ & 0.062 & 0.004 & $(0.055,0.069)$ & 0.063 & 0.006 & $(0.056,0.074)$ \\
$\sigma_{1 m}$ & 0.006 & 0.0018 & $(0.003,0.009)$ & 0.006 & 0.002 & $(0.003,0.011)$ & 0.007 & 0.004 & $(0.002,0.015)$ \\
$\sigma_{2 m}$ & 0.095 & 0.1779 & $(0.000,0.394)$ & 0.023 & 0.008 & $(0.010,0.036)$ & 0.031 & 0.007 & $(0.020,0.041)$ \\
$\sigma_{3 m}$ & 0.013 & 0.0146 & $(0.000,0.037)$ & 0.008 & 0.001 & $(0.006,0.011)$ & 0.009 & 0.001 & $(0.006,0.011)$ \\
\hline
\end{tabular}

Effective Computing Time

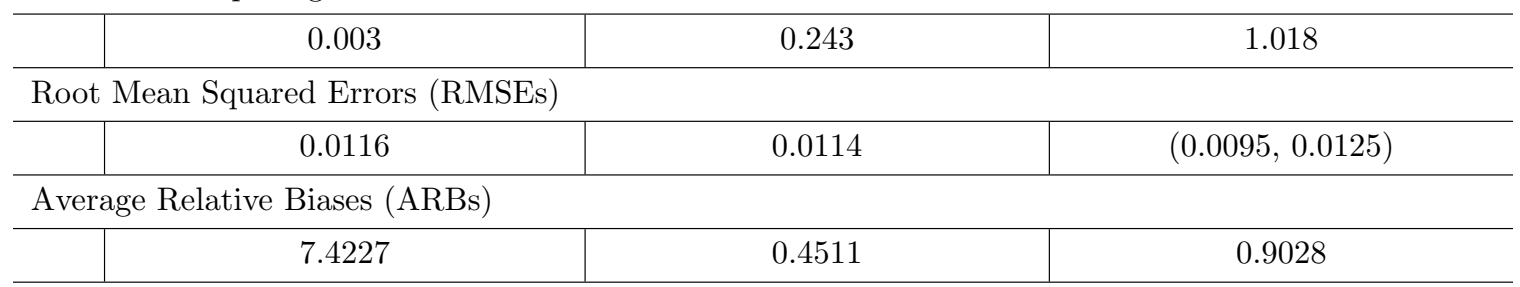


Table 1.6 Logmarginal Likelihood: Extremely Nonlinear Case

\begin{tabular}{c|ccc}
\hline $\mathrm{p}$ & $\mathrm{CDKF}$ & $\mathrm{GMF}$ & $\mathrm{PF}$ \\
\hline 0.1 & 218.02 & 365.35 & 365.92 \\
0.5 & 218.18 & 365.47 & 365.56 \\
0.9 & 218.39 & 365.54 & 365.14 \\
\hline
\end{tabular}

creates a large set of new Gaussian mixture components.

Table 1.6 reports the logmarginal likelihoods for the calibration of the extremely nonlinear case. The CDKF with PM-MH has significantly lower values of the logmarginal likelihood than those from the GMF and the PF. The logmarginal likelihood obtained from the GMF with PM-MH is nearly the same as those from the PF with PM-MH.

\section{Near Linear Case}

As a near-linear case, the calibration maintains the same parameter values as those in the benchmark case, except that it increases $\sigma$ to 50 and decreases $\sigma_{\mu}$ to 0.007 . The Frisch labor elasticity implied by $\sigma$ is 0.047 . The low Frisch labor elasticity and low $\sigma_{\mu}$ introduce a near linearity to the economy by significantly reducing the role of second-order terms in the approximated model solution.

Figure 1.3 and Table 1.7 show that all of the estimation procedures exactly capture the true values of parameters. Since the model is nearly linear, the estimation results might be the same as those from the Kalman filter with PM-MH. Table 1.7 shows that the RMSEs of the filtered state variables from the CDKF are nearly the same as the RMSEs obtained from the GMF, implying that this parameter specification does not allow a significant role in the omitted higher-order terms in the CDKF. The RMSEs of the CDKF and the GMF are both inside the $95 \%$ confidence interval of the RMSEs from the PF. Although the ARBs for the GMF is the smallest among the filtering methods, the ARBs for the CDKF and the PF are not much different 
Figure 1.3: Posterior Distribution: Nearly Linear Case
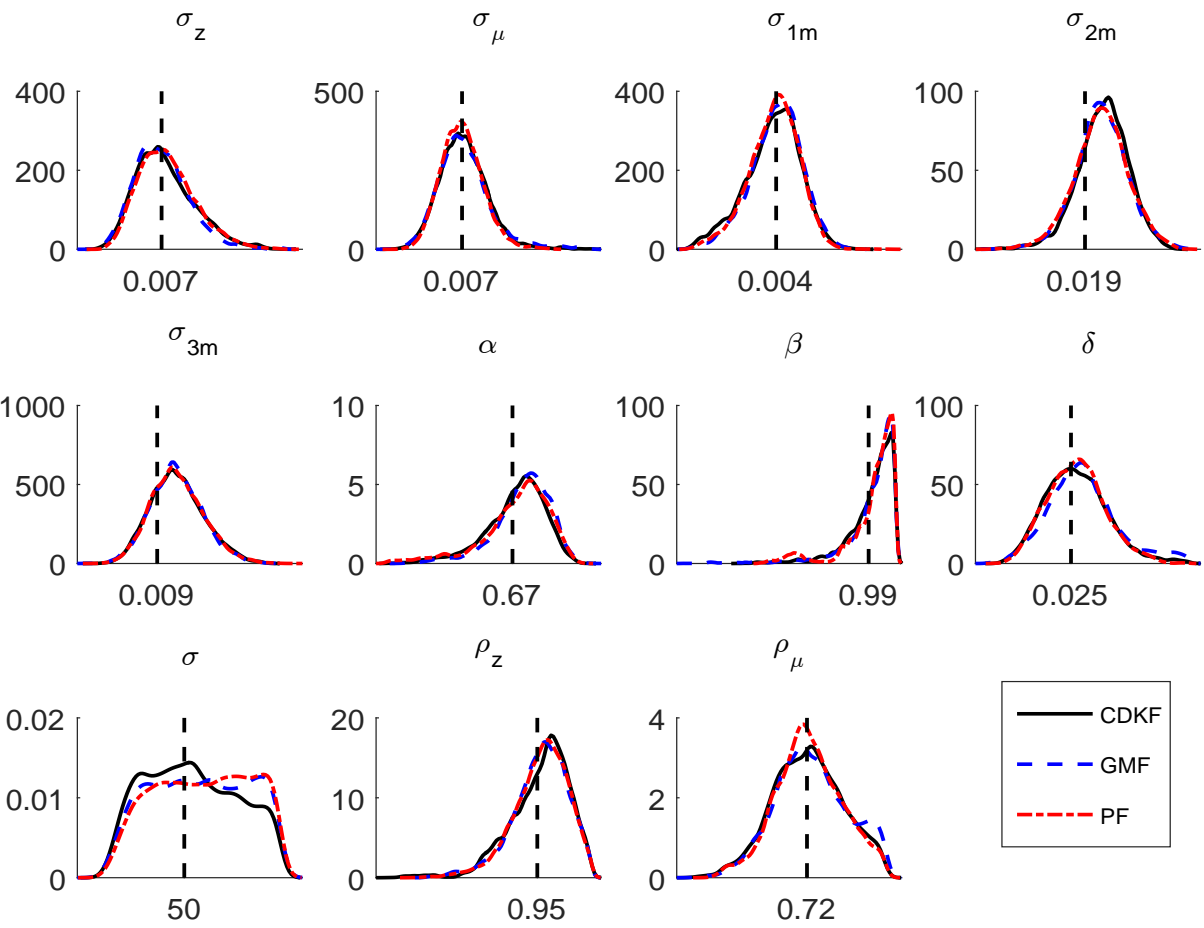

Notes. The vertical dashed line represents true parameter values.

Table 1.7 Posterior Distribution: Nearly Linear Case

\begin{tabular}{c|ccc|ccc|ccc}
\hline & \multicolumn{3}{|c|}{ CDKF } & \multicolumn{3}{c|}{ GMF } & \multicolumn{3}{c}{ PF } \\
\hline & Mean & S.D. & Prob. Interval & Mean & S.D. & Prob. Interval & Mean & S.D. & Prob. Interval \\
\hline$\alpha$ & 0.68 & 0.086 & $(0.535,0.824)$ & 0.701 & 0.081 & $(0.548,0.810)$ & 0.681 & 0.101 & $(0.468,0.808)$ \\
$\beta$ & 0.991 & 0.013 & $(0.969,0.999)$ & 0.991 & 0.01 & $(0.972,0.999)$ & 0.991 & 0.01 & $(0.965,0.999)$ \\
$\delta$ & 0.026 & 0.009 & $(0.011,0.041)$ & 0.028 & 0.007 & $(0.016,0.042)$ & 0.026 & 0.006 & $(0.016,0.037)$ \\
$\sigma$ & 54.12 & 9.944 & $(37.418,70.830)$ & 58.12 & 24.74 & $(19.150,95.911)$ & 59.71 & 24.31 & $(20.653,96.351)$ \\
$\rho_{z}$ & 0.953 & 0.023 & $(0.914,0.992)$ & 0.953 & 0.025 & $(0.906,0.990)$ & 0.953 & 0.025 & $(0.908,0.989)$ \\
$\rho_{\mu}$ & 0.724 & 0.183 & $(0.416,0.999)$ & 0.736 & 0.126 & $(0.533,0.955)$ & 0.727 & 0.114 & $(0.541,0.925)$ \\
$\sigma_{z}$ & 0.007 & 0.002 & $(0.004,0.010)$ & 0.007 & 0.002 & $(0.005,0.010)$ & 0.007 & 0.002 & $(0.005,0.010)$ \\
$\sigma_{\mu}$ & 0.007 & 0.001 & $(0.005,0.009)$ & 0.007 & 0.001 & $(0.005,0.010)$ & 0.007 & 0.001 & $(0.005,0.009)$ \\
$\sigma_{1 m}$ & 0.004 & 0.001 & $(0.002,0.006)$ & 0.004 & 0.001 & $(0.002,0.006)$ & 0.004 & 0.001 & $(0.002,0.006)$ \\
$\sigma_{2 m}$ & 0.022 & 0.004 & $(0.015,0.029)$ & 0.022 & 0.005 & $(0.014,0.029)$ & 0.022 & 0.005 & $(0.014,0.030)$ \\
$\sigma_{3 m}$ & 0.01 & 0.001 & $(0.008,0.012)$ & 0.01 & 0.001 & $(0.008,0.011)$ & 0.01 & 0.001 & $(0.008,0.011)$ \\
\hline
\end{tabular}

Effective Computing Time

\begin{tabular}{c|c|c|c}
\hline & 0.002 & 0.014 & 0.292 \\
\hline \multicolumn{1}{c|}{ Root Mean Squared Errors (RMSEs) } \\
\hline 0.0105 & 0.0105 & $(0.0101,0.0110)$ \\
\hline \multicolumn{2}{l}{ Average Relative Biases (ARBs) } & & \\
\hline & 0.1214 & 0.1186 & 0.1222 \\
\hline
\end{tabular}


Table 1.8 Logmarginal Likelihood: Nearly Linear Case

\begin{tabular}{c|ccc}
\hline $\mathrm{p}$ & $\mathrm{CDKF}$ & $\mathrm{GMF}$ & $\mathrm{PF}$ \\
\hline 0.1 & 809.10 & 809.21 & 808.85 \\
0.5 & 809.18 & 809.32 & 809.04 \\
0.9 & 809.31 & 809.33 & 809.15 \\
\hline
\end{tabular}

from those for the GMF. Most of the parameters have the similar asymptotic standard deviations, except for $\sigma$ possibly due to the non-Gaussianity of its posterior distribution. Moreover, Table 1.8 shows that the logmarginal likelihoods implied by filtering methods are almost the same as each other.

\section{Third-Order Approximation with Stochastic Volatility}

Although the second-order approximation embeds risk factors into the model, it does not allow time-varying risk in the model. To allow for time-varying risk and investigate the dynamic effects of the risk shocks, it is required to consider the third-order approximation. Since the 2008 financial crisis, there have been lots of studies investigating the real effect of the risk shocks, including policy risk shocks, on economic activities (Fernández-Villaverde et al., 2011; Mumtaz and Zanetti, 2013; Born and Pfeifer, 2014a; Fernández-Villaverde et al., 2015; Mumtaz and Theodoridis, 2015; Bonciani and van Roye, 2016; Leduc and Liu, 2016; Basu and Bundick, 2017). However, most of the works rely on the calibration results and a few of them use the simulated method of moments (SMM) to estimate the models. The advantage of the SMM is that it is computationally cheaper than likelihood-based methods. However, it is not always obvious which moments to select to estimate the parameters of the model, and the estimation results are sensitive to the chosen moments (Mumtaz and Zanetti, 2013).

In this section, we estimate the benchmark neoclassical growth model with stochastic volatility using the PM-MH with Gaussian-based filters. We add the 
Figure 1.4: Posterior Distribution: Benchmark with stochastic volatility
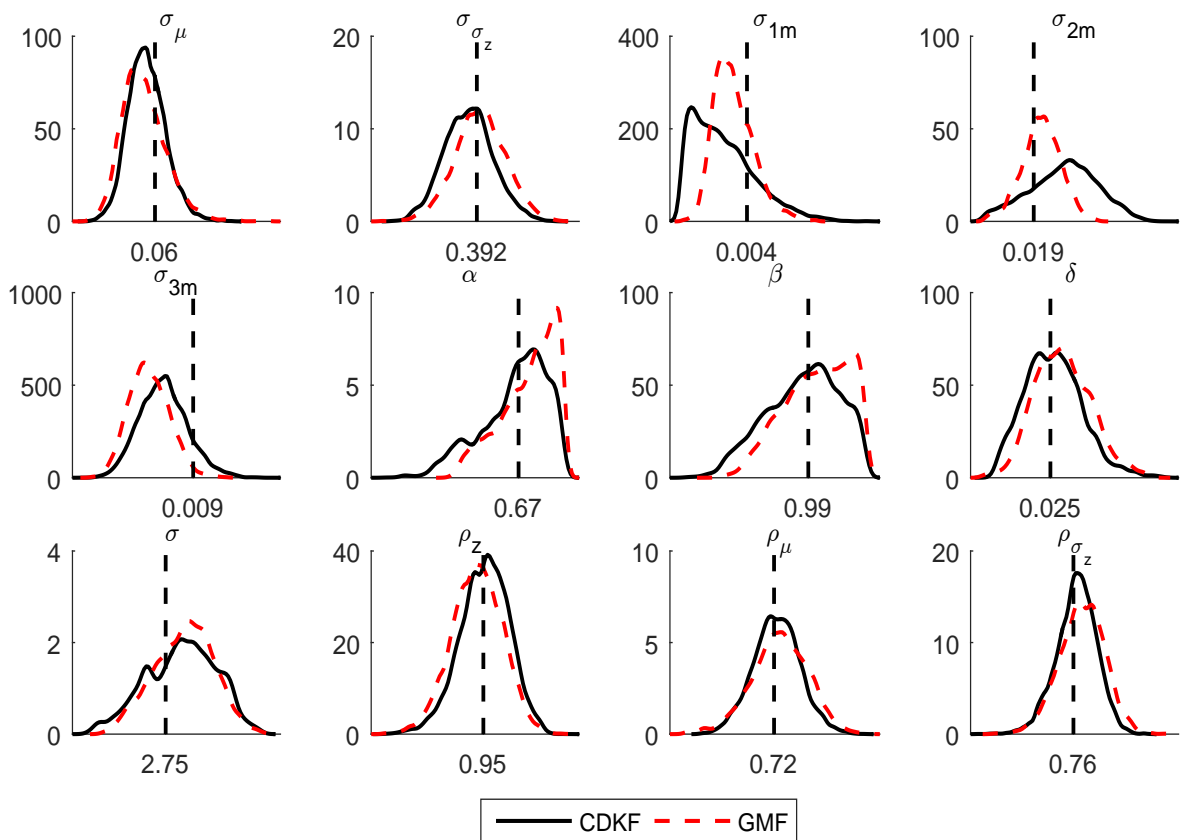

Notes. The vertical dashed line represents true parameter values.

stochastic volatility to the aggregate technology shock process in the benchmark model. Following Andreasen (2013), our specification of stochastic volatility for the technology shock process can be represented as

$$
\begin{gathered}
\log z_{t}=\sigma_{z, t} \log \lambda_{t} \\
\log \lambda_{t+1}=\rho_{z} \frac{\sigma_{z, t}}{\sigma_{z, t+1}} \log \lambda_{t}+\eta_{z, t+1} \\
\log \left(\frac{\sigma_{z, t+1}}{\tilde{\sigma}_{z}}\right)=\rho_{\sigma_{z}} \log \left(\frac{\sigma_{z, t}}{\tilde{\sigma}_{z}}\right)+\eta_{\sigma_{z}, t+1}
\end{gathered}
$$

where $\eta_{z, t+1} \sim i . i . d N(0,1)$ and $\eta_{\sigma_{z}, t+1} \sim i . i . d N\left(0, \sigma_{\sigma_{z}}\right)$.

We use three observable variables $y_{t}=\left[c_{t}, i_{t}, h_{t}\right]^{\prime}$ for the estimation. The approximated solution has four state variables $x_{t}=\left[k_{t}, \mu_{t}, \lambda_{t}, \sigma_{z, t}\right]^{\prime}$. We increase the total number of MCMC iterations to 100,000 to assure convergence to the stationary distribution of this more complicated model and use the last 50,000 draws to compute 
Table 1.9 Posterior Distribution: Benchmark with stochastic volatility

\begin{tabular}{c|ccc|ccc}
\hline & \multicolumn{4}{|c}{ CDKF } & \multicolumn{3}{c}{ GMF } \\
\hline & Mean & S.D. & Prob. Interval & Mean & S.D. & Prob. Interval \\
\hline$\alpha$ & 0.6623 & 0.0167 & $(0.634,0.690)$ & 0.6906 & 0.055 & $(0.581,0.758)$ \\
$\beta$ & 0.989 & 0.0021 & $(0.985,0.993)$ & 0.9915 & 0.0056 & $(0.982,0.999)$ \\
$\delta$ & 0.0256 & 0.0018 & $(0.022,0.029)$ & 0.0274 & 0.0056 & $(0.019,0.037)$ \\
$\sigma$ & 2.8334 & 0.1137 & $(2.642,3.024)$ & 2.8483 & 0.1605 & $(2.565,3.099)$ \\
$\rho_{z}$ & 0.9505 & 0.0131 & $(0.928,0.973)$ & 0.9474 & 0.0106 & $(0.930,0.964)$ \\
$\rho_{\mu}$ & 0.7264 & 0.0531 & $(0.637,0.816)$ & 0.735 & 0.074 & $(0.609,0.852)$ \\
$\rho_{\sigma_{z}}$ & 0.7637 & 0.0222 & $(0.726,0.801)$ & 0.7702 & 0.0274 & $(0.723,0.812)$ \\
$\sigma_{\mu}$ & 0.0581 & 0.0063 & $(0.047,0.069)$ & 0.0575 & 0.0053 & $(0.050,0.067)$ \\
$\sigma_{\sigma_{z}}$ & 0.3813 & 0.0346 & $(0.323,0.439)$ & 0.3965 & 0.0335 & $(0.341,0.452)$ \\
$\sigma_{1 m}$ & 0.0026 & 0.0017 & $(0.000,0.005)$ & 0.0032 & 0.0012 & $(0.002,0.005)$ \\
$\sigma_{2 m}$ & 0.0303 & 0.0226 & $(0.000,0.068)$ & 0.0218 & 0.0073 & $(0.009,0.034)$ \\
$\sigma_{3 m}$ & 0.0081 & 0.0008 & $(0.007,0.009)$ & 0.0076 & 0.0006 & $(0.007,0.009)$ \\
\hline
\end{tabular}

Effective Computing Time

\begin{tabular}{c|c|c}
\hline & 0.003 & 0.178 \\
\hline \multicolumn{2}{c|}{ Root Mean Squared Errors (RMSEs) } \\
\hline \multicolumn{2}{c}{2.0355} & 1.5177 \\
\hline \multicolumn{2}{c}{ Average Relative Biases (ARBs) } \\
\hline & 0.203 & 0.0925 \\
\hline
\end{tabular}

Table 1.10 Logmarginal Likelihood: Benchmark with stochastic volatility

\begin{tabular}{c|cc}
\hline $\mathrm{p}$ & CDKF & GMF \\
\hline 0.1 & 472.20 & 510.31 \\
0.5 & 472.56 & 510.53 \\
0.9 & 472.71 & 509.73 \\
\hline
\end{tabular}

point estimates. We set the threshold $\eta_{\text {limit }}$ to 1 to make the GMF feasible. As we did in the previous exercises, we test the ability of the PM-MH with the Gaussian-based filters based on simulated data with 100 observations. Since the PF with PM-MH requires the severe computational burden, we do not consider exploring the properties of the PF with PM-MH. To simulate the model, we follow the calibration in the benchmark case without stochastic volatility. Additionally, we set the persistence level $\rho_{\sigma_{z}}$ and the standard deviation of structural shock $\sigma_{\sigma_{z}}$ in the volatility process to 0.76 and 0.392 , respectively. These values are based on Leduc and Liu (2016). The mean of volatility $\tilde{\sigma_{z}}$ is set to 0.007 which is a calibrated value of $\sigma_{z}$ in the benchmark case.

Table 1.9 shows that the GMF gives more accurate filtered state variables than 
the CDKF. The RMSEs for the CDKF is 2.035 and the RMSEs for the GMF is 1.517. These are much larger than the values obtained from the benchmark model without stochastic volatility. That implies that the omitted higher-order terms in the CDKF seem to play a significant role in capturing realized true state variables in the benchmark with stochastic volatility. Figure 1.4 reveals that most of the posterior distributions closely capture the true parameter values because the true values are inside the credible sets. The overall implication is that although the nonlinearity implied by the benchmark model with stochastic volatility deteriorates the performance of the CDKF, it does not create a significant effect on capturing true parameters. These results are consistent with the findings based on a New Keynesian model in Andreasen (2013). In addition, the GMF with PM-MH complements the CDKF with PM-MH by mixing Gaussian distributions. The asymptotic sandwich variances for the CDKF are similar to those for the GMF in most of the parameters, except for $\alpha$, $\beta, \delta$, and $\sigma_{2 m}$

However, it is cautious that the good estimation results for $\rho_{\sigma_{z}}$ and $\sigma_{\sigma_{z}}$ might be obtained under a condition where the coefficient of variation (CV) of the volatility process is large enough to avoid a model close to the one of constant volatility (Jacquier et al., 2002). ${ }^{10}$ If the CV is close to 0 , then it is hard to distinguish between a DSGE with stochastic volatility and a DSGE with constant volatility so that the likelihood over $\rho_{\sigma_{z}}$ and $\sigma_{\sigma_{z}}$ could be less informative. ${ }^{11}$ In this exercise, the CV with $\rho_{\sigma_{z}}=0.76$ and $\sigma_{\sigma_{z}}=0.392$ is 0.438 . Since the estimates for $\rho_{\sigma_{z}}$ and $\sigma_{\sigma_{z}}$ are important to investigate the effect of volatility shock on economic activities, we should be careful of the dynamics of stochastic volatility process.

${ }^{10}$ The coefficient of variation is calculated as

$$
C V=\frac{\operatorname{var}(h)}{E[h]^{2}}=\exp \left(\frac{\sigma_{\sigma_{z}}^{2}}{1-\rho_{\sigma_{z}}^{2}}\right)-1
$$

\footnotetext{
${ }^{11}$ When setting $\rho_{\sigma_{z}}=0.9$ and $\sigma_{\sigma_{z}}=0.135$, the $\mathrm{CV}$ is 0.1 . In that case, the estimation result for $\rho_{\sigma_{z}}$ becomes poorer, though the result for $\sigma_{\sigma_{z}}$ is not bad. The estimation results are given in Appendix A.5.
} 
The effective computing time of the CDKF with PM-MH and the GMF with PM-MH is dramatically increased compared to the benchmark case of second-order approximated solution. This is mainly driven by the fact that solving DSGE models up to third order is more computationally expensive than solving the models up to second order. Table 1.10 reports the logmarginal likelihoods for the benchmark model with stochastic volatility. The logmarginal likelihood implied by the CDKF with PM-MH is slightly lower than that obtained by the GMF with PM-MH.

\subsection{Application to 'Real' Data}

In this section, we estimate the parameters of the neoclassical growth model for the U.S. quarterly data from 1991:Q1 to 2015:Q3. This empirical exercise is based on real GDP, real investment, and nonfarm business hours worked. The details of the data are given in Appendix A.6. We detrend the data using an HP filter. This exercise allows us to investigate which filter with PM-MH provides a better explanation of the real data and test whether the CDKF and the GMF are still valid for the real data or only work for the Monte Carlo study. To make this exercise empirically realistic, we set the labor share $\alpha$, the discount factor $\beta$, and the depreciation rate $\delta$ to $0.67,0.99$, and 0.025, respectively, and only consider eight of the model parameters as unknown. To avoid the particle degeneracy problem of the PF, we allow measurement errors to absorb no less than $5 \%$ of the variance of the corresponding observable variables. We obtain 150,000 draws from the posterior distributions, after 50,000 burin-in periods.

Figure 5 shows that all of the posteriors have the similar shapes with small differences. When we look at Table 11, it is more clear that all of the filters with PMMH have the similar posterior means with the similar posterior probability intervals. The estimates of $\sigma$ ranges from 0.17 to 0.19 , implying that the Frisch labor supply elasticity ranges from 12.28 to 13.72 . The estimated $\rho_{z}$ indicates that the aggregate 

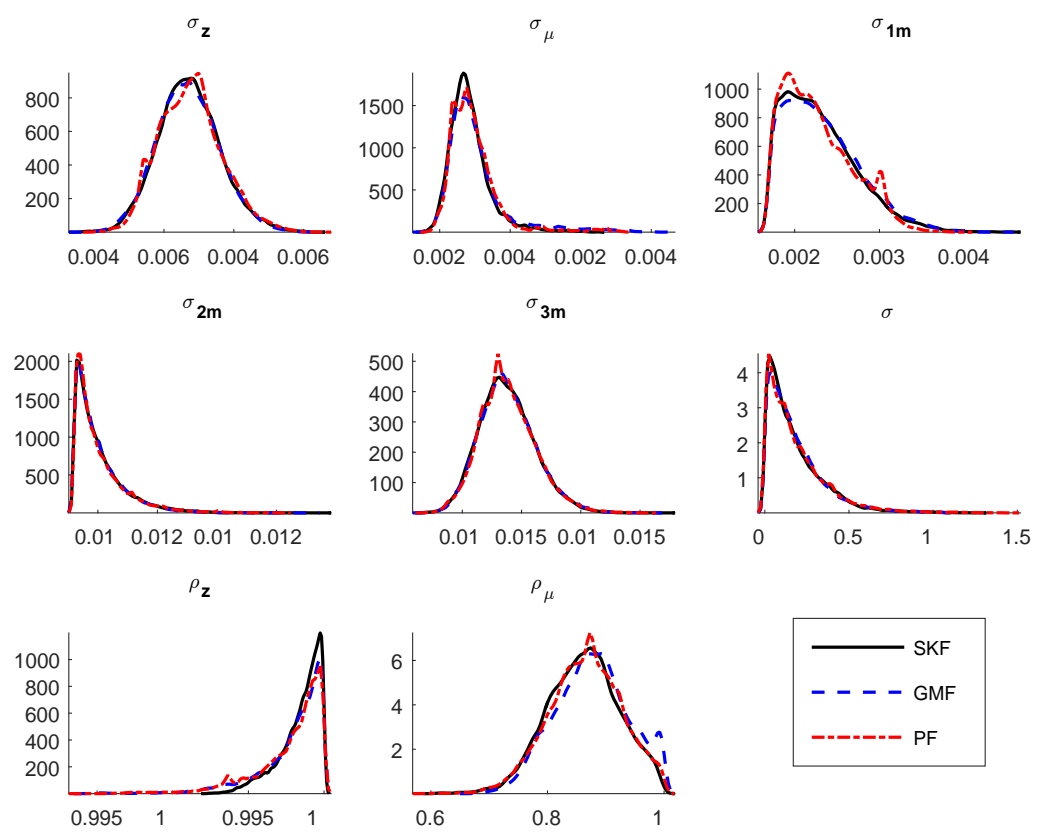

Figure 1.5: Posterior Distribution Based on Real Data

technology shock is highly persistent. However, the investment-specific technology shock is less persistent than the aggregate technology shock. Table 12 reports the logmarginal likelihood among the filtering methods. It shows that although the GMF has slightly larger values of the logmarginal likelihood, these values are not much different from those of the $\mathrm{CDKF}$ and the $\mathrm{PF}$, implying that both the CDKF and the GMF provide a comparable explanation of the real data over the PF. Finally, to assess whether the small differences in point estimates documented in Table 11 are big or small, we calculate standard deviations of simulated data (output, consumption, investment, and hours worked), based on the given estimates for each filter. Table 13 presents the standard deviations of the real data and the simulated data at the mean of the posterior distribution of the parameters. It shows that all of the estimates, obtained from the three filters, tend to generate the similar standard deviations of the simulated data. In addition, these values tend to match the standard deviations of the real data well, except for the standard deviations of hours worked.

Overall, the CDKF and the GMF work well with real data. However, contrary 
Table 1.11 Posterior Distribution Real Data

\begin{tabular}{c|ccc|ccc|ccc}
\hline & \multicolumn{4}{|c|}{ CDKF } & \multicolumn{3}{c|}{ GMF } & \multicolumn{3}{c}{ PF } \\
\hline & Mean & S.D. & Prob. Interval & Mean & S.D. & Prob. Interval & Mean & S.D. & Prob. Interval \\
\hline$\sigma$ & 0.1778 & 0.1674 & $(0.0109,0.4962)$ & 0.1826 & 0.1634 & $(0.0111,0.5121)$ & 0.1863 & 0.1801 & $(0.0124,0.5355)$ \\
$\rho_{z}$ & 0.9994 & 0.0005 & $(0.9982,0.9999)$ & 0.9991 & 0.0009 & $(0.9973,0.9999)$ & 0.9991 & 0.0009 & $(0.9973,0.9999)$ \\
$\rho_{\mu}$ & 0.8617 & 0.0614 & $(0.7602,0.9639)$ & 0.8758 & 0.0626 & $(0.7721,0.9847)$ & 0.8647 & 0.0623 & $(0.7582,0.9686)$ \\
$\sigma_{z}$ & 0.0044 & 0.0004 & $(0.0037,0.0051)$ & 0.0043 & 0.0004 & $(0.0036,0.0051)$ & 0.0044 & 0.0004 & $(0.0037,0.0052)$ \\
$\sigma_{\mu}$ & 0.0014 & 0.0003 & $(0.0011,0.0019)$ & 0.0015 & 0.0004 & $(0.0011,0.0025)$ & 0.0015 & 0.0004 & $(0.0011,0.0020)$ \\
$\sigma_{1 m}$ & 0.0023 & 0.0004 & $(0.0018,0.0031)$ & 0.0023 & 0.0004 & $(0.0018,0.0031)$ & 0.0023 & 0.0004 & $(0.0018,0.0030)$ \\
$\sigma_{2 m}$ & 0.009 & 0.0004 & $(0.0086,0.0099)$ & 0.009 & 0.0004 & $(0.0086,0.0098)$ & 0.009 & 0.0004 & $(0.0086,0.0099)$ \\
$\sigma_{3 m}$ & 0.0115 & 0.0009 & $(0.0101,0.0131)$ & 0.0115 & 0.0009 & $(0.0101,0.0132)$ & 0.0115 & 0.0009 & $(0.0101,0.0132)$ \\
\hline
\end{tabular}

Table 1.12 Logmarginal Likelihood

\begin{tabular}{c|ccc}
\hline $\mathrm{p}$ & $\mathrm{CDKF}$ & $\mathrm{GMF}$ & $\mathrm{PF}$ \\
\hline 0.1 & 925.87 & 926.02 & 925.81 \\
0.5 & 926.18 & 926.22 & 926.06 \\
0.9 & 926.35 & 926.41 & 926.27 \\
\hline
\end{tabular}

Table 1.13 Standard Deviations $\left(\sigma_{x}\right)$ (Real data vs Simulated data)

\begin{tabular}{l|c|c|c|c|c}
\hline \hline & & Output & Consumption & Investment & Hours worked \\
\hline Real data & & 0.867 & 0.686 & 3.088 & 1.382 \\
Simulated data (CDKF) & & 1.168 & 0.412 & 2.089 & 0.400 \\
Simulated data (GMF) & $\sigma_{x}$ & 1.177 & 0.412 & 2.116 & 0.407 \\
Simulated data (PF) & & 1.179 & 0.413 & 2.115 & 0.405 \\
\hline \hline
\end{tabular}

to the Monte Carlo study, we don't know the data generating mechanism in the exercise with the real data. If the data generating mechanism is unknown, then the parametric method potentially can have misspecification issues. However, the GMF is still useful in the sense that the Gaussian mixture approximation can be used to approximate any distributions of structural shocks and measurement errors so that the GMF is generalizable. In addition, the concept of Binomial Gaussian mixture can be applied to set up a proposal density for the particle filter. This would make the particle filter more efficient. In future works, it would be also interesting to apply Bayesian nonparametric methods to estimate the unknown distribution function of structural shocks in a general state-space model, based on Dirichlet processes. All of these strategies, however, are beyond the scope of this paper. 


\subsection{Conclusion}

The recent financial crisis has highlighted the importance of nonlinear features including risks, uncertainty shocks, rare disasters, and structural changes. Following this strand of the literature, this study provides a novel and an alternative approach to posterior inference on parameters in a nonlinear Dynamic Stochastic General Equilibrium (DSGE) model and discusses their convergence to the true invariant density. Especially, we implement two Gaussian-based filters to evaluate the likelihood and construct the posterior distribution in the application of neoclassical growth model: the CDKF and the GMF, and then compare the proposed approaches with the PF with PM-MH regarding estimation accuracy and computational efficiency. Finally, we apply the methods to a medium-class of DSGE model solved to second order with pruning.

The overall implication is that the estimation accuracy of the CDKF with PM$\mathrm{MH}$ and the GMF with PM-MH is comparable to that of the PF with PM-MH in the benchmark and the nearly linear case. These results are based on the RMSEs of filtered state variables and the ARBs of posterior means. Although the CDKF creates biased estimates of several parameters in the extremely nonlinear case, its computational cost is much less than the PF with PM-MH, and the GMF complements the poor performance of the CDKF by mixing Gaussian distributions. Surprisingly, the Gaussian-based filters with PM-MH show reasonably accurate posterior inference on parameters in the benchmark case with stochastic volatility capturing true parameter values in most cases. However, they might fail to identify the true parameter values in the stochastic volatility process when the $\mathrm{CV}$ of the volatility process is close to zero. We also note that all of the Gaussian-based filters in this study are much more efficient than the PF in terms of effective computing time. 


\section{Chapter 2}

\section{House Price Uncertainty, Business Cycles, and Welfare}

\section{$2.1 \quad$ Introduction}

I think that the central issue in the economic situation right now is the housing market. It is the continued uncertainty about house prices and housing activity which is creating financial stress, is affecting consumer wealth and consumer expectations and causing the stress we are seeing in the economy. So my suggestion would be in the near term to focus on issues related to housing.

Ben S. Bernanke, JULY 15, 2008

Since the Great Recession of 2008-2009, which was partially driven by a collapse in the housing market, uncertainty surrounding housing has been an important issue for economists and policymakers. Recent studies have shown that uncertainty itself is a source of economic fluctuations and slows down economic activity. However, house price uncertainty has two different forces in the housing market (see Han, 2010, 2013); hence these forces potentially have asymmetric impacts on economic activity over the business cycle. On the one hand, uncertainty about house prices makes home-owning 
and mortgage debt risky, and hence reduce house prices due to a fall in demand for housing. On the other hand, households faced with house price uncertainty might use an early purchase strategy to hedge against future housing consumption risk. Since a primary residence comprises about two-thirds of the median homeowner's assets, the unexpected change in house price uncertainty could have significant impacts on households' consumption through a wealth effects channel and a collateral constraints channel. In addition, changes in house price uncertainty can either positively or negatively affect the real-options values of residential investment projects, and hence affect firms' decisions on residential investment in a different way.

The relationship between uncertainty and economic activity has been widely documented in a partial equilibrium framework (Leland, 1968; Hartman, 1976; Bernanke, 1983; Abel, 1983; Kimball, 1990; Carroll et al., 2006; Bloom, 2009). Building on this work, recent dynamic stochastic general equilibrium (DSGE) models investigate the effects of uncertainty on business cycle fluctuations in a general equilibrium setting (Fernández-Villaverde et al., 2011; Gómez-González et al., 2013; Born and Pfeifer, 2014a,b; Cesa-Bianchi and Corugedo, 2018; Fernández-Villaverde et al., 2015; Mumtaz and Theodoridis, 2015; Leduc and Liu, 2016; Bonciani and van Roye, 2016; Basu and Bundick, 2017). Most of these studies rely mainly on the markup channel with sticky prices, as emphasized by Basu and Bundick (2017). Based on this key mechanism, Born and Pfeifer (2014a) and Fernández-Villaverde et al. (2015) investigate policy uncertainty impacts on economic activity, Leduc and Liu (2016) propose search frictions in the labor market, and Cesa-Bianchi and Corugedo (2018) and Bonciani and van Roye (2016) introduce financial frictions in the model.

However, there has been relatively little work on the transmission channels and the spillover effects of house price uncertainty in a general equilibrium framework, though there have been extensive studies that explore the risk-return relationship 
in the finance literature using asset pricing models. ${ }^{1}$ Importantly, some of the real estate literature in finance investigate the effects of house price risk on homeowners' decision making in partial equilibrium (Banks et al., 2004; Sinai and Souleles, 2005; Han, 2010, 2013). Moreover, a large body of literature in macroeconomics that investigates the relationship between the housing market and economic activity has focused on analyzing the direct effects of house price shocks on aggregate macroeconomic variables, but the role played by changes in house price uncertainty is new to the literature. This paper attempts to fill this gap by investigating the asymmetric effects of house price uncertainty on economic activity over the business cycle, finding its possible sources, identifying transmission mechanisms, conducting welfare analysis, and finally evaluating optimal policy rules.

We first estimate a house price process in the United States (U.S.) that is characterized by time-varying stochastic volatility in its shocks. This specification allows us to distinguish between house price shocks and house price uncertainty shocks. The estimated volatility that we call house price uncertainty shows time-variation and tends to rise in housing boom and bust periods. Following Fernández-Villaverde et al. (2015), we interpret the unexpected changes in the volatility as unexpected shocks to uncertainty about house prices. Next, we estimate a benchmark vector autoregression of the U.S. economy augmented with the house price uncertainty that we extracted in the first step and compute impulse response functions (IRFs) of several macroeconomic variables to an identified house price uncertainty shock. We allow the benchmark VAR model to be regime-switching because of well-known business cycle asymmetries (Hamilton, 1989; Owyang et al., 2005). From this exercise, we find that the house price uncertainty shock has asymmetric impacts on the U.S. economy in that it leads to a decline in consumption, residential investment, house prices, and

\footnotetext{
${ }^{1}$ Frank Knight (1921) distinguishes between risk and uncertainty. He describes risk as events where the distribution is known over a set of events. On the other hand, he defines uncertainty as conditions where economic agents are unable to predict the likelihood of events. In this study, we will refer to uncertainty as a mixture of risk and uncertainty following Bloom (2014).
} 
mortgage debt, but an increase in rent to price ratio in recessionary regimes. However, the opposite responses occur in expansionary regimes for residential investment, house prices, mortgage debt, and rent to price ratio.

The second step is to investigate uncertainty shocks in a nonlinear New Keynesian DSGE model with a housing sector (Iacoviello and Neri, 2010). The objective is to reproduce the asymmetric dynamic behaviors generated by the house price uncertainty shock in the MS-VAR analysis. This model allows us to add a set of second-moment structural shocks that are interpreted as exogenous uncertainty shocks. We then investigate the propagation mechanism of each uncertainty shock. The model is solved and estimated based on a third order approximation. We first estimate the model without stochastic volatility processes by embedding the Central Difference Kalman filter (CDKF) into the Monte Carlo Markov Chain (MCMC) algorithm (Andreasen, 2013; Binning and Maih, 2015; Noh, 2018). As a second step, we match observations of the U.S. economy in terms of second moments and first- and second-order autocorrelations to estimate parameters of the stochastic volatility processes. Although this two-step procedure does not guarantee efficiency, it allows us not only to use full information maximum likelihood to estimate key structural parameters, but also to capture the role of stochastic volatility processes in explaining fluctuations of the main macroeconomic variables with reasonable computational burden. Consistent with the existing literature, the impulse responses for the given estimated parameters demonstrate that uncertainty shocks dampen economic activity. They reduce consumption, business investment, residential investment, housing prices, household debt, and output, though business investment initially increases after the shocks.

The variance decomposition analysis shows that uncertainty shocks to investmentspecific technology, technology in the nonhousing sector, housing preference, and inflation target play significant roles in explaining the variance in house prices, implying that these shocks could possibly be the main sources of the house price uncertainty 
shocks. The theoretical model illustrates that supply-side uncertainty shocks produce negative impacts on consumption, residential investment, housing prices, and household debt. The model implies that the supply-side uncertainty shocks induce precautionary motives, negative real-options effects on residential investment, and financial and debt risk effects on housing demand. These results are mainly driven by the uncertainty shocks to investment-specific technology, capturing the key empirical findings obtained from the recessionary regimes in the MS-VAR analysis, except that they increase inflation and interest rates. The reason for the increase in inflation is that firms and labor unions optimally choose higher prices and wages due to the upward pricing biases with sticky prices and wages (Fernández-Villaverde et al., 2015). The rise in inflation leads to a rise in interest rates. In contrast, we find that among demand-side uncertainty shocks, the uncertainty shocks to housing preference have positive impacts on residential investment, house prices, and household debt. This resembles the empirical findings when the economy is in expansionary regimes. However, the other demand-side uncertainty shocks, mainly driven by the uncertainty shocks to inflation target, have negative impacts on these variables.

In this study, we explore transmission mechanisms of house price uncertainty by focusing on a financial risk effect, a housing consumption hedging effect, and a debt risk effect. The financial risk and debt risk effects are associated with the fact that when households have a strong desire for nonhousing consumption or when they face high borrowing costs, they tend to have a weak desire for housing and mortgage debt. This result implies that higher uncertainty about housing prices lowers house prices in recessionary regimes. On the contrary, the hedging effect against future housing consumption risk implies that households faced with house price uncertainty are more likely to pay a higher price in expansionary regimes. All of these effects are related to decisions about home owning and hence housing prices. By deriving an analytic relationship between uncertainty and the returns to owning a home under simplifying 
assumptions, we show that model-simulated data based on uncertainty shocks create the financial risk effect, the hedging effect, and the debt risk effect. The sign and magnitude of the relationship depends on the relative forces of these three effects.

We numerically evaluate welfare changes when there is an increase in uncertainty based on the following four different specifications: (1) allowing high uncertainty for all shocks; (2) high uncertainty for supply-side shocks; (3) high uncertainty for demand-side shocks; (4) high uncertainty for each structural shock. The results show that the conditional compensating variations, based on the increases in all uncertainty, are negative. The negative values driven by both lenders and borrowers, implying that they can exploit an uncertain economy by optimally re-allocating resources. Lenders mainly benefit from an increase in demand-side uncertainty, but borrowers benefit from an increase in supply-side uncertainty. Finally, we evaluate the optimal LTV ratio and the optimal interest rate rules over a range of mean uncertainty. The optimal LTV ratio is 0.68 for the given estimated parameters. It responds countercyclically to housing preference uncertainty and monetary policy uncertainty, but it responds procyclically to uncertainty about investment-specific technology. The optimal interest rate rule does not respond to interest-rate inertia, but moderately responds to inflation and weakly responds to GDP growth.

The paper is organized as follows. Section 2 describes transmission mechanisms of uncertainty shocks. Section 3 estimates house price uncertainty, and describes some stylized facts. Section 4 reports the MS-VAR evidence. Section 5 introduces the New Keynesian DSGE model with a housing market, and discusses numerical implementation for the model solution and estimation. Section 6 presents the main results. Section 7 implements a welfare analysis and evaluates the optimal policy rules. Section 8 concludes. 


\subsection{Uncertainty Transmission Mechanism}

In this section, we discuss possible channels through which uncertainty affects economic activity within the housing market. These include real-options channels, channels of a convex marginal revenue product of capital and a convex marginal profit, precautionary savings channels, and housing demand channels via a financial risk effect, a housing consumption hedging effect, and a debt risk effect. The first three channels have been widely discussed in DSGE models (Born and Pfeifer, 2014a; Cesa-Bianchi and Corugedo, 2018; Fernández-Villaverde et al., 2015; Mumtaz and Theodoridis, 2015; Leduc and Liu, 2016; Bonciani and van Roye, 2016; Basu and Bundick, 2017). However, the financial risk effect, the hedging effect, and the debt risk effect, all of which are driven by house price uncertainty, have not been emphasized in DSGE models. Although all of these channels are important in a partial equilibrium sense, their effects on aggregate economic activity have not yet been studied in a general equilibrium framework.

First, the real-options channels are associated with the right to undertake a new investment project which is irreversible or partially irreversible when economic agents are faced with uncertainty about future cash flows, interest rates, and the price of capital (Bernanke, 1983). For example, the opportunity to invest in capital in the future at a lower price (or the right to sell capital in the future at a higher price) is a real call option (or a real put option). Depending on the real-options value of the investment project, uncertainty might have a negative effect or a positive effect on the investment decision. If the real call option to undertake investment in the future is more valuable than the real put option, then uncertainty has a negative effect on investment. However, partially irreversible investment could lead uncertainty to have a positive effect on the put option value and hence make more investment desirable. Since this study has an interest in investigating the effect of house price uncertainty, its direct effect is mainly associated with residential investment, not 
with business investment. In our model, which will be discussed in the later section, for the given predetermined capital, the real-options value might be associated with expected returns on capital in the housing sector, adjustment costs on capital and capacity utilization, and stochastic shocks.

Second, the convex marginal revenue product of capital, regarding output prices and total factor productivity (TFP), induces a positive relationship between uncertainty and investment (Oi, 1961; Hartman, 1972; Abel, 1983). This is known as the Oi-Hartman-Abel effect. However, this channel holds when firms freely expand to exploit good outcomes and contract to avoid bad outcomes without adjustment costs. In our model, the capacity utilization potentially allows flexibility to adjust capital inputs. On the contrary, the convex marginal profit with sticky prices generates a negative relationship between uncertainty and investment as an increase in uncertainty leads firms to optimally choose higher prices and hence increases markups over marginal costs (Born and Pfeifer, 2014a). This leads to a fall in output, and hence a fall in investment.

Third, the precautionary savings channels suggest that when economic agents are faced with higher uncertainty about future income, they consume less, but increase savings and labor supply to hedge against future negative shocks (Carroll et al., 2006). In a closed economy, the increase in savings directly induces an increase in investment. The recent work by Basu and Bundick (2017) explains the macroeconomic effect of uncertainty shocks based on the economic agents' precautionary behaviors in a general equilibrium model. They describe that uncertainty shocks in a standard neoclassical growth model cannot produce comovement of aggregate macroeconomic variables, such as output, investment, hours worked, and consumption. However, the shocks can generate comovement in a New Keynesian model through markup channels characterized by sticky prices and wages based on Rotemberg-type nominal rigidities. Similarly, Mumtaz and Theodoridis (2015) show that uncertainty shocks with Calvo- 
type nominal rigidities increase price and wage dispersion, which creates less efficient aggregate production, requiring more labor input. This increases real wages, marginal costs, and inflation. In our paper, uncertainty shocks could be amplified through the markup of final goods over wholesale goods and the markup between the wage cost for wholesale firms and households' wage. ${ }^{2}$

Finally, an increase in house price uncertainty affects housing demand through three channels: the financial risk effect, the housing consumption hedging effect, and the debt risk effect. The financial risk effect is associated with the investment decision on financial assets and future asset returns. When investors in financial markets are faced with uncertainty about asset prices, they reduce their demand for financial assets as the financial risk of future asset returns increases. Since it is not easy to diversify house price uncertainty, the negative effect of house price uncertainty on housing demand tends to be particularly large (Caplin et al., 1997). Different from the financial risk effect, the hedging effect is associated with future housing consumption. In this case, housing is considered as a necessary consumption good, not a financial asset (Henderson et al., 1983; Brueckner, 1997). This describes that high house price uncertainty leads households to own houses to hedge against future housing consumption risk. (Berkovec and Fullerton, 1992; Ortalo-Magné and Rady, 2002). Thus, both channels potentially have contrasting effects on housing demand, and hence housing prices. In addition, uncertainty about house prices might lead mortgage debt to be risky. Since mortgage borrowing opportunities rely on the fluctuations in the price of housing collateral, house price uncertainty leads the economy to become exposed to high uncertainty about borrowing opportunities and borrowing costs. Risk-averse households reduce their demand for housing consumption and hence mortgage debt to avoid this risk exposure. Because U.S. mortgage debt constitutes roughly one-third of all private debts and two-thirds of consumer debts, the debt risk effect could play

\footnotetext{
${ }^{2}$ In this study, the price stickiness occurs at the retail level following Bernanke et al. (1999)
} 
an important role in households' economic activity.

The changes in housing prices (directly or indirectly) driven by all of the channels mentioned above, affects the borrowing opportunities and thus the level of consumption through wealth effects and collateral constraints (Kiyotaki et al., 1997; Iacoviello and Neri, 2010; Liu et al., 2013).

\subsection{Evidence of Time-Varying House Price Uncer- tainty}

In this section, we estimate the time-varying uncertainty of the U.S. housing price in a model where economic agents form rational expectations of future housing prices using currently available information. We define house price uncertainty by the standard deviation of the unpredictable component of house price that is commonly considered as a measure of risk in the financial literature. Based on this measure of house price uncertainty, we investigate the evidence of its time-variation and a relationship between house price uncertainty and real economic activity.

\subsubsection{House Price Uncertainty}

The challenge in this empirical analysis is to measure house price uncertainty. First, we can directly use economic agents' expectations about future house prices obtained from surveys. Although the survey from the University of Michigan Survey of Consumers reports the expected changes in future home values with variances, the data span is not long enough for the analysis. As alternatives, it can be measured by either the cross-sectional variability or the time-series volatility of actual housing price data.

Micro-based financial studies for the housing market have proxied house price uncertainty in various ways. For example, uncertainty about housing prices is proxied by location indicators reflecting that housing prices in high-priced cities tend to have 
high standard deviations (Ioannides and Rosenthal, 1994; Green and Vandell, 1999). Others use variability in past house price history (Sinai and Souleles, 2005), or fiveyear conditional variance predicted by a GARCH model (Han, 2010). However, the location proxies and the variability approach based on past house price history are vulnerable to control market conditions and capture time-varying characteristics of house price uncertainty. Considering that economic agents make decisions on housing demand and supply based on the current and expected future economic conditions, a measure of house price uncertainty should vary over time. In addition, GARCH models do not distinguish between innovations to the level of house price and to its volatility. Since house price uncertainty might be driven mainly by exogenous shocks, this study considers estimating a stochastic volatility model that allows us to gauge the independent effect of shocks to house price uncertainty on economic activity.

To create the measure of house price uncertainty, this study assumes that economic agents make forecasts about future housing prices based on past housing prices, aggregate economic conditions, and policy information. Changes in the forecast error variance will then proxy for uncertainty. We use the one period lagged Shiller real house price as past house price history. The aggregate economic conditions include the real income and unemployment rates. Finally, the 3-month treasury bill rate is assumed to be policy information. The real income is the real compensation per hour in the nonfarm business sector from FRED. The unemployment rate is the civilian unemployment rate from FRED. The 3-month treasury bill is from FRED. We use quarterly-based data that covers the period from 1975Q2 to 2015Q4. The Shiller house price is deflated by CPI from FRED.

Specifically, we define the detrended log of Shiller real house price at time t, $h p_{t}$, as a function of the lagged detrended log of Shiller real house price, $h p_{t-1}$, lagged 3 -month treasury bill rate, $t b_{t-1}$, lagged detrended log of real income, $i n c_{t-1}$, lagged unemployment rate, uemp $p_{t-1}$, and unpredicted shock, $\varepsilon_{h p, t}$. The volatility, $\sigma_{h p, t}$, is 
modeled as a function of one-period lagged volatility, $\sigma_{h p, t-1}$ and an exogenous shock $u_{\sigma, t}$. We detrend the log of real house price and real income using the HodrickPrescott (HP) filter. ${ }^{3}$ Based on the above specification, we estimate the following law of motion for the housing price and stochastic volatility process:

$$
\begin{aligned}
\mathrm{hp}_{t}-\overline{h_{p}} & =\rho_{h p}\left(\mathrm{hp}_{t-1}-\overline{h p}\right)+\phi_{r} t b_{t-1}+\phi_{i n c}\left(i n c_{t-1}-i \bar{n} c\right) \\
& +\phi_{u}\left(u e m p_{t-1}-u e \bar{m} p\right)+\exp \left(\sigma_{h p, t}\right) \varepsilon_{h p, t}, \varepsilon_{h p, t} \sim N(0,1)
\end{aligned}
$$

where $\overline{h p}$ is the mean detrended log of real house price, $i \bar{n} c$ is the mean detrended log of real income, ue $\bar{m} p$ is the mean unemployment rate, $\rho_{h p}$ is the persistence of the $\log$ of real house price, and $\sigma_{h p, t}$ is the log of the standard deviation of an innovation to the real house price. $\sigma_{h p, t}$ follows an $\mathrm{AR}(1)$ process:

$$
\sigma_{h p, t}=\left(1-\rho_{\sigma}\right) \sigma_{h p}+\rho_{\sigma} \sigma_{h p, t-1}+\left(1-\rho_{\sigma}^{2}\right)^{1 / 2} \sigma_{u} u_{\sigma, t}, u_{\sigma, t} \sim N(0,1)
$$

where $\rho_{\sigma}$ is the persistence of the $\log$ of the standard deviation of an innovation to the real house price, $\sigma_{h p}$ determines the $\log$ of the average standard deviation of the house price shock, and $\sigma_{u}$ is the standard deviation of the house price uncertainty shock. Note that, in each period, the house price shocks, captured by $\varepsilon_{h p, t}$, cause housing prices to deviate from expectations conditional on past house price history, aggregate economic conditions, and the policy condition. Although these sudden shocks could be driven by either the demand or the supply side, we cannot identify each side of the shocks from the above specification. The shock $u_{\sigma, t}$ that affects the house price volatility are considered to be the house price uncertainty shock.

\footnotetext{
${ }^{3}$ We tried other detrending methods, including the linear detrending and the first log difference. However, the main results do not change.
} 
Table 2.1 Prior and Posterior Distribution

\begin{tabular}{c|cc|cc}
\hline & \multicolumn{2}{|c|}{ posterior } & \multicolumn{2}{c}{ prior } \\
\hline & median & $90 \%$ credible set & distribution & hyper \\
\hline$\rho_{h p}$ & 0.966 & $(0.935,0.989)$ & Beta & $(0.8,0.1)$ \\
$\phi_{r}$ & -0.222 & $(-0.319,-0.119)$ & Normal & $\left(\hat{\phi}_{r}^{O L S}, 0.1\right)$ \\
$\phi_{i n c}$ & 0.076 & $(0.014,0.141)$ & Normal & $\left(\hat{\phi}_{i n c}^{O L S}, 0.1\right)$ \\
$\phi_{u}$ & -0.008 & $(-0.057,0.038)$ & Normal & $\left(\hat{\phi}_{u}^{O L S}, 0.1\right)$ \\
$\rho_{\sigma}$ & 0.948 & $(0.888,0.983)$ & Beta & $(0.8,0.1)$ \\
$\sigma_{h p}$ & -0.449 & $(-0.876,-0.004)$ & Uniform & $(-10,10)$ \\
$\sigma_{u}$ & 0.190 & $(0.124,0.281)$ & Gamma & $(1.0,1.0)$ \\
\hline
\end{tabular}

\subsubsection{Estimation}

We estimate the parameters and stochastic volatility of equations (2.1) and (2.2) using particle MCMC (Fernández-Villaverde and Rubio-Ramírez, 2005; Andrieu et al., 2010). Since the model with the mean equation (2.1) and the stochastic volatility process (2.2) is not a linear state-space, we cannot obtain a closed form of the marginal likelihood. In such a case, we need to use an approximation method to evaluate the likelihood. The beauty of the particle filter with MCMC is that the difficult problem of sampling from $p\left(\theta, \sigma_{h p, 1: T} \mid h p_{1: T}\right)$ can be reduced to sampling from $p\left(\theta \mid h p_{1: T}\right)$ where $\theta$ is a set of parameters including coefficients and standard deviations of our mean equation and stochastic volatility process. By evaluating the approximately exact marginal likelihood $\hat{p}\left(h p_{1: T} \mid \theta\right)$ through the sequential Monte Carlo method and embedding them into the MCMC algorithm, we can sample $\theta$ and $\sigma_{h p, 1: T}$ from $p\left(\theta, \sigma_{h p, 1: T} \mid h p_{1: T}\right)$. Under the random walk Metropolis-Hastings algorithm, we accept drawn samples of $\theta^{*}$ and $\sigma_{h p, 1: T}^{*}$ with probability

$$
\min \left[1, \frac{\hat{p}\left(h p_{1: T} \mid \theta^{*}\right) p\left(\theta^{*}\right)}{\hat{p}\left(h p_{1: T} \mid \theta\right) p(\theta)}\right] \text {, where } \theta \text { is from previous samples. }
$$

Table 2.1 reports prior distributions for the parameters of the house price process with posterior medians. For the persistence parameters, $\rho_{h p}$ and $\rho_{\sigma}$, we impose a 
Beta prior with the mean of 0.8 and standard deviation of 0.1 . The shape of this prior restricts the parameter values to lie between 0 and 1 . For the log of the mean standard deviation of the house price shock, $\sigma_{h p}$, we impose a Uniform prior ranging from -10 to 10. For each coefficient of control variables, we employ a Normal prior. The mean of the prior for each coefficient is set to the OLS estimate of equation (2.1) without stochastic volatility, and the standard deviation is set to 0.1. For the standard deviation of the house price uncertainty shock, $\sigma_{u}$, we use a Gamma prior with the mean of 1.0 and standard deviation of 1.0 to ensure that this parameter is positive.

The posterior medians are obtained by using the random walk Metropolis-Hastings sampling algorithm. After the initial 10,000 burn-in draws, we use 50,000 draws to evaluate the posterior distribution of the parameters and uncertainty measures. The scaling matrix of the proposal density is adaptively adjusted following Vihola (2012) to achieve the appropriate acceptance rate around 0.4.

Table 2.1 shows that the parameter estimates for the persistence, $\rho_{h p}$, of the detrended $\log$ of real house price and the persistence, $\rho_{\sigma}$, of the volatility are 0.966 and 0.948 . They are highly persistent. The coefficients for other control variables are consistent with economic reasoning. For example, the parameter, $\phi_{r}$ is negative, suggesting that house price is negatively correlated with the monetary policy rate $\left(\phi_{r}<0\right)$. In addition, consistent with what one might expect, the house price is positively correlated with the detrended $\log$ of income $\left(\phi_{i n c}>0\right)$, but negatively correlated with the unemployment rate $\left(\phi_{u}<0\right)$. Finally, the average standard deviation of the house price shock is 0.638 percentage point $(\exp (-0.449))$ and the standard deviation of the house price uncertainty shock is 0.190 . A positive onestandard deviation shock $u_{\sigma, t}$ increases the standard deviation of the house price shock to 0.877 percentage points $\left(\exp \left(-0.449+\left(1-0.948^{2}\right)^{(1 / 2)}\right)\right)$. 


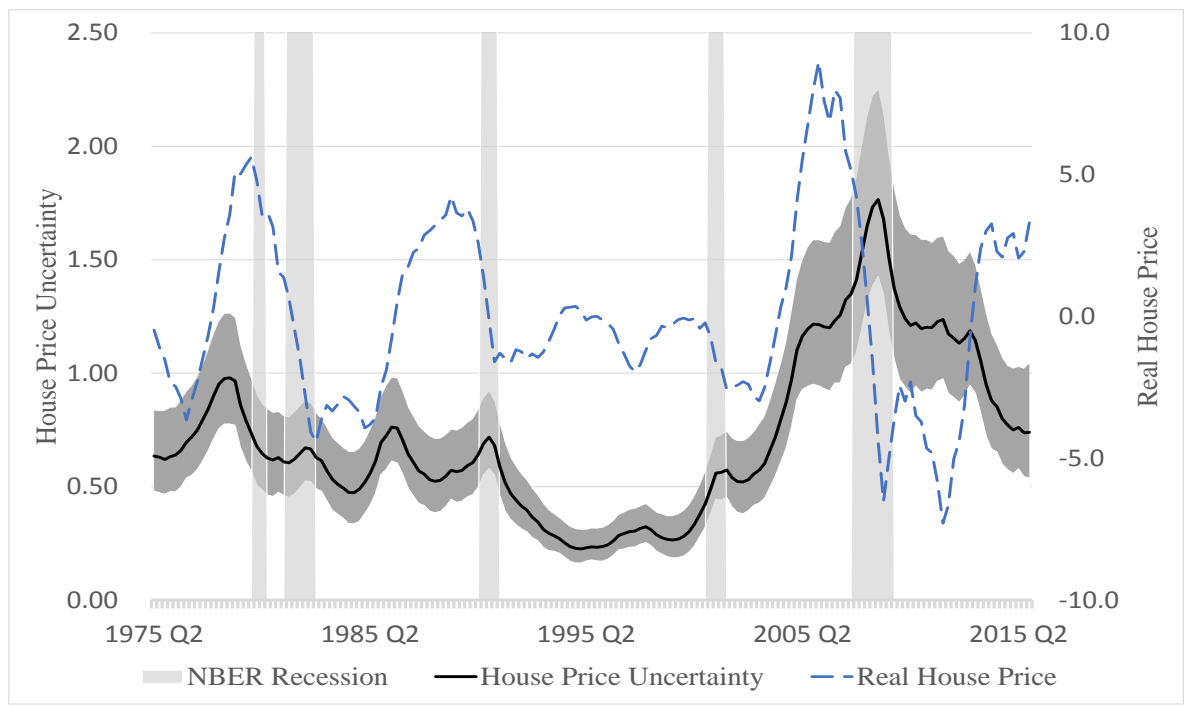

Notes: A blue dashed line is the HP-filtered real house price.

Figure 2.1: House Price Uncertainty

\subsubsection{Basic Empirical Regularities}

In this section, we investigate basic empirical regularities by linking house price uncertainty to business cycles and consumers' expectations about the future housing market. We take advantage of the housing price process and the stochastic volatility process, specified by equations (2.1) and (2.2), and apply the particle MCMC to draw the unobservable house price volatility conditional on our whole sample. We define the median smoothed volatility conditional on the posterior medians of the sampled parameters as house price uncertainty. We calculate the cross-correlation between house price uncertainty and economic activity and the three-year rolling correlation between house price uncertainty and consumers' opinions about the future housing market dynamics.

Figure 2.1 displays the posterior median and the $90 \%$ posterior probability intervals of the smoothed house price volatility, $\exp \left(\sigma_{h p}\right)$, over the sample. We also plot the percentage deviation of real house price from the trend and shade NBER recessionary periods. This shows time-varying evidence of house price uncertainty. The evolution of house price uncertainty corresponds to boom and bust cycles of house 
prices. It allows us to build an analytic narrative of house price uncertainty.

Figure 2.1 shows that house price uncertainty significantly rises in 1976-1978 and reaches a peak at nearly 0.9 percentage point in 1978. Case (1994) points out that there was a house price boom in California in the late 1970s; the growth rate of housing prices outpaced income growth. However, real house prices reach a low point in the early 1980s with an increase in house price uncertainty. The housing boom also occurred in California, Northeast, Hawaii, Seattle, and Washington, D.C. in the late 1980s, creating a rise in house price uncertainty. However, real house prices reach a trough in the early 1990s, creating slightly high uncertainty as shown in Figure 2.1. Figure 2.1 also shows that house price uncertainty has increased recently, reflecting the recent boom and bust cycles of the housing market. In 2001, real house prices appreciate much faster than before. The percentage deviation of real house price from the trend reaches nearly double-digit levels in 2005. In addition, the 2008 financial crisis caused by the unprecedented collapse in the housing market has created the highest uncertainty in housing prices over the sample periods. Consistent with other macroeconomic indicators of uncertainty, such as the volatility of stock markets, bond markets, exchange rates, and GDP growth, the volatility of housing prices tends to rise in recessions.

To find some stylized relationship between house price uncertainty and economic activities, we investigate the cross-correlations between house price uncertainty, obtained from the previous econometric model, and aggregate macroeconomic variables. We also calculate the cross-correlations between house price uncertainty and housing market-related variables, including housing prices and mortgage debt. We take the first log-difference of the aggregate macroeconomic and housing market-related variables. Figure 2.2 shows that house price uncertainty anticipates lower levels of consumption, residential investment, and output. The negative correlations for residential investment and output appear with similar magnitudes at each lag, but the 


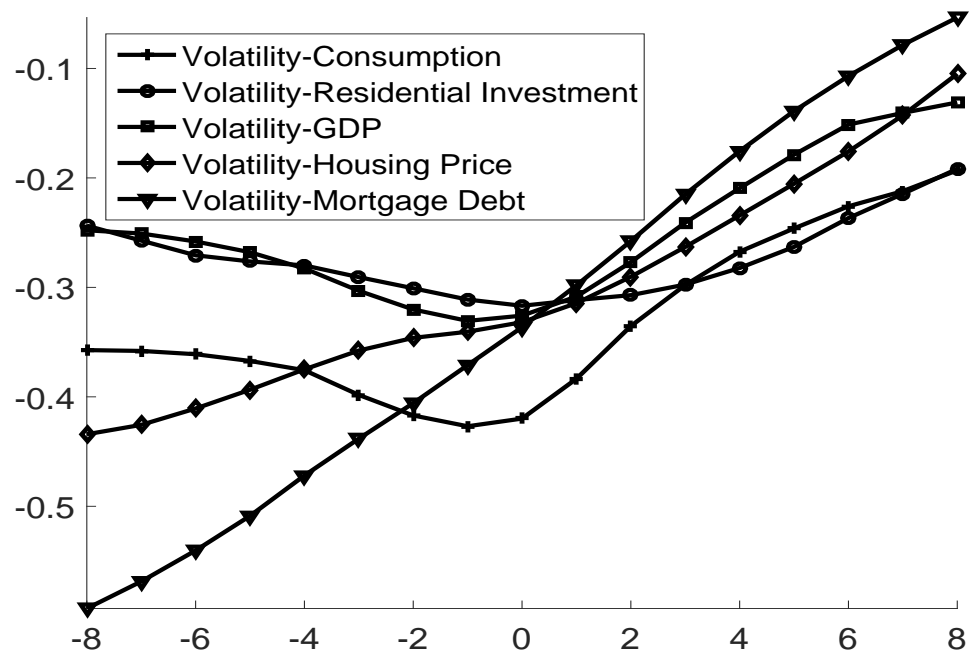

Figure 2.2: Cross-Correlation

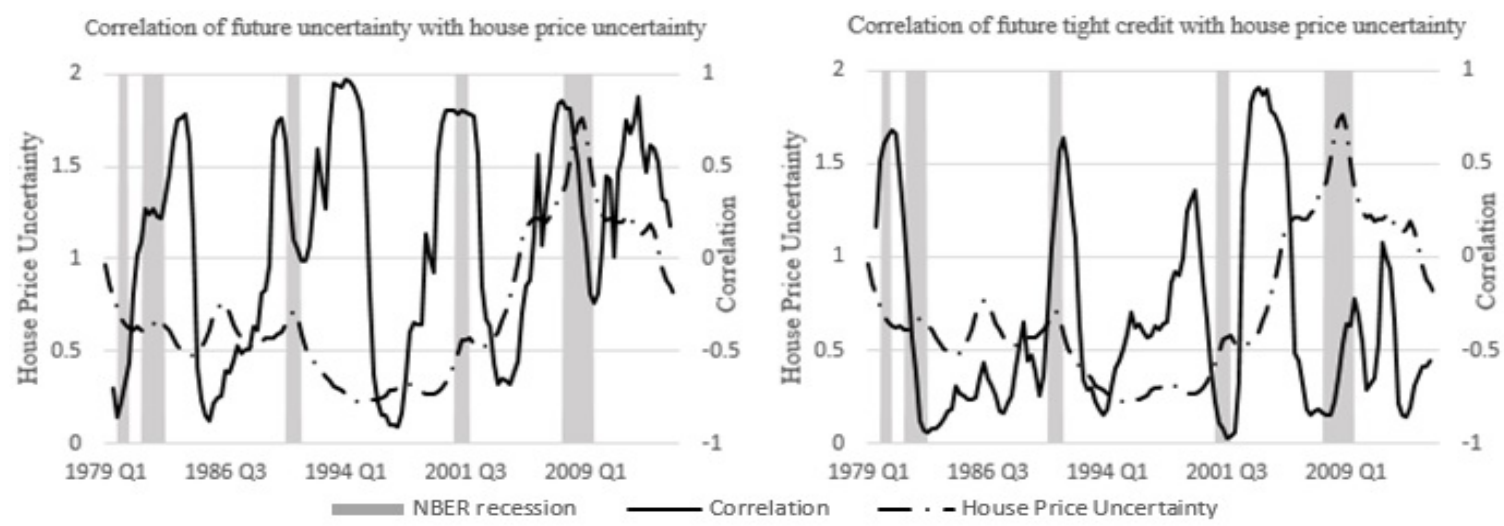

Figure 2.3: Three-year rolling correlation

correlation for consumption is stronger than that of residential investment and output. Figure 2.2 also shows that house price uncertainty leads housing prices and mortgage debt. The contemporaneous correlations between the aggregate macroeconomic variables and house price uncertainty range from around -0.3 to -0.45 . For the case of housing prices and mortgage debt, the contemporaneous correlation is around 0.3. To summarize, house price uncertainty is countercyclical and tends to lead the economic activities.

We also investigate how house price uncertainty is related to housing market dynamics using survey data from the University of Michigan Survey of Consumers. 
This survey reports the consumers' opinion as to whether it is a good time or a bad time to buy a house and the reasons for the opinions. Based on the fraction of respondents citing (1) Bad Time to Buy a House due to Uncertain future and (2) Good Time to Buy a House due to Rising Interest Rates as the reasons for the opinions about good or bad buying conditions, we construct two variables: (1) future uncertainty and (2) future tight credit. The first variable could be associated with the financial risk and debt risk effect when consumers are faced with high house price uncertainty. On the other hand, the second variable could be associated with the housing consumption hedging effect when they are faced with high future housing consumption risk.

To analyze the relationship between house price uncertainty and consumer expectations about the future housing market, we calculate three-year moving average correlations between house price uncertainty and the two variables. Panel A of Figure 2.3 shows that house price uncertainty is positively correlated with future uncertainty in the early 1980s, early 1990s, early 2000s, and late 2000s, all of which are in recessionary periods, implying that a consumers' negative opinion due to future uncertainty about the future housing market could be related to the financial risk and debt risk effect that house price uncertainty had over these periods. On the other hand, Panel B of Figure 2.3 shows that house price uncertainty is positively correlated with future tight credit in the phase of housing booms such as those in the late 1970s and mid 2000s. This implies that a consumers' positive opinion due to the future tight credit might be affected by the housing consumption hedging effect that house price uncertainty had over housing boom periods. 


\subsection{Empirical VAR Analysis}

\subsubsection{Benchmark MS-VAR Model}

To investigate macroeconomic consequences of house price uncertainty shocks empirically, this section considers a quarterly two-lags MS-VAR model with time-varying coefficients and heteroskedastic errors. ${ }^{4}$ This allows us to characterize macroeconomic fluctuations when our economy has structural changes, and to investigate asymmetric effects of house price uncertainty shocks. The model is represented as follows:

$$
\begin{gathered}
Y_{t}=c_{s_{t}}+\sum_{i=1}^{2} \phi_{s_{t}, i} Y_{t-i}+\Sigma_{s_{t}}^{1 / 2} \varepsilon_{t}, \quad \varepsilon_{t} \sim N(0,1), \\
P=\left[\begin{array}{ll}
p_{11} & p_{12} \\
p_{21} & p_{22}
\end{array}\right],
\end{gathered}
$$

where $S_{t}$ indexes regimes, $P$ denotes a transition probability matrix with components $p_{i j}=p\left(s_{t}=j \mid s_{t-1}=i\right) . \quad \Sigma_{s_{t}}=A_{s_{t}}^{-1} A_{s_{t}}^{-1^{\prime}}$ where $A_{s_{t}}$ denotes the contemporaneous relationship between reduced-form shocks, $c_{s_{t}}$ is a constant term, and $Y_{t}$ is a vector of endogenous variables. This specification allows us to not only investigate the regimedependent effects of house price uncertainty shocks on economic activities but also improves the fit of the model. The Bayesian Deviance information criterion (DIC) of a non-regime-dependent VAR model is -2395.8 while the DIC with two regimes is -2603.1, implying that the MS-VAR model is preferred over the constant VAR model. Note that the DIC proposed in Spiegelhalter et al. (2002) is a generalization of the Akaike information criterion in the sense that it consists of the goodness of fit and

\footnotetext{
${ }^{4}$ We choose a lag length following Towbin and Weber (2015). The main results are robust regardless of the lag length. Increasing the number of lags from two to four does not change our main results. Although a large number of regressors allows us to use more information, estimates could have large variances.
} 
the complexity of the model. ${ }^{5}$ The number of regimes is restricted to 2-state Markov chain. Although the model with three regimes is slightly preferred to the model with two regimes in terms of the DIC, we can use a large enough number of samples at each regime with two regimes and show clear asymmetric implications of house price uncertainty shocks. ${ }^{6}$

To estimate the model, we use quarterly data from 1975:1 to 2015:4 of nine endogenous variables in the following order: filtered real housing price volatility, real personal consumption expenditure, real private residential investment, consumer price index (CPI) inflation, real Shiller house price index, 3-month treasury bill rate, mortgage rate, the size of mortgage debt, and rent to price ratio. Since the smoothed house price volatility has information over all the sample periods so that it might be more likely to be endogenously determined, we use the filtered volatility in this exercise (Fernández-Villaverde et al., 2015). We estimate the model using a MCMC algorithm and compute credible sets from the posterior. The detailed procedure is described in Appendix B.2. House price uncertainty shocks are identified by standard Cholesky decomposition. The ordering is based on the assumption that housing price uncertainty is exogenous, implying that it reacts to changes in the other variables only with a quarter lag. The main results are robust regardless of the ordering of house price uncertainty shocks. We also find no evidence that any of endogenous variables Granger-cause the filtered house price uncertainty.

The consumption and residential investment are obtained from the Bureau of Economic Analysis (BEA). The Shiller's housing price index, CPI, Treasury bill rate, and mortgage debt are from FRED. We use the nominal contract rate on the purchases of existing single-family homes from the Federal Housing Financing Agency (FHFA)

\footnotetext{
${ }^{5}$ The DIC is calculated as $-2 \bar{D}-2 p_{D}$ where the goodness of fit of the model $\bar{D}$ is the expected $\log$ of likelihood $E\left[\ln L\left(\theta_{i}\right)\right]$ and the complexity of the model $p_{D}$ is the expected log of likelihood $E\left[\ln L\left(\theta_{i}\right)\right]$ minus the $\log$ of likelihood evaluated at the posterior mean $\ln L\left(E\left[\theta_{i}\right]\right) . \quad E\left[\ln L\left(\theta_{i}\right)\right]$ is approximated as $\frac{1}{N} \sum_{i}\left(\ln L\left(\theta_{i}\right)\right)$, and $\ln L\left(E\left[\theta_{i}\right]\right)$ is approximated as $\ln L\left(\frac{1}{N} \sum_{i} \theta_{i}\right)$.

${ }^{6}$ The DIC with two regimes is -2603.1 , while the DIC with three regimes is -2768.6. However, the asymmetric effects of house price uncertainty shocks are robust regardless of the number of regimes.
} 


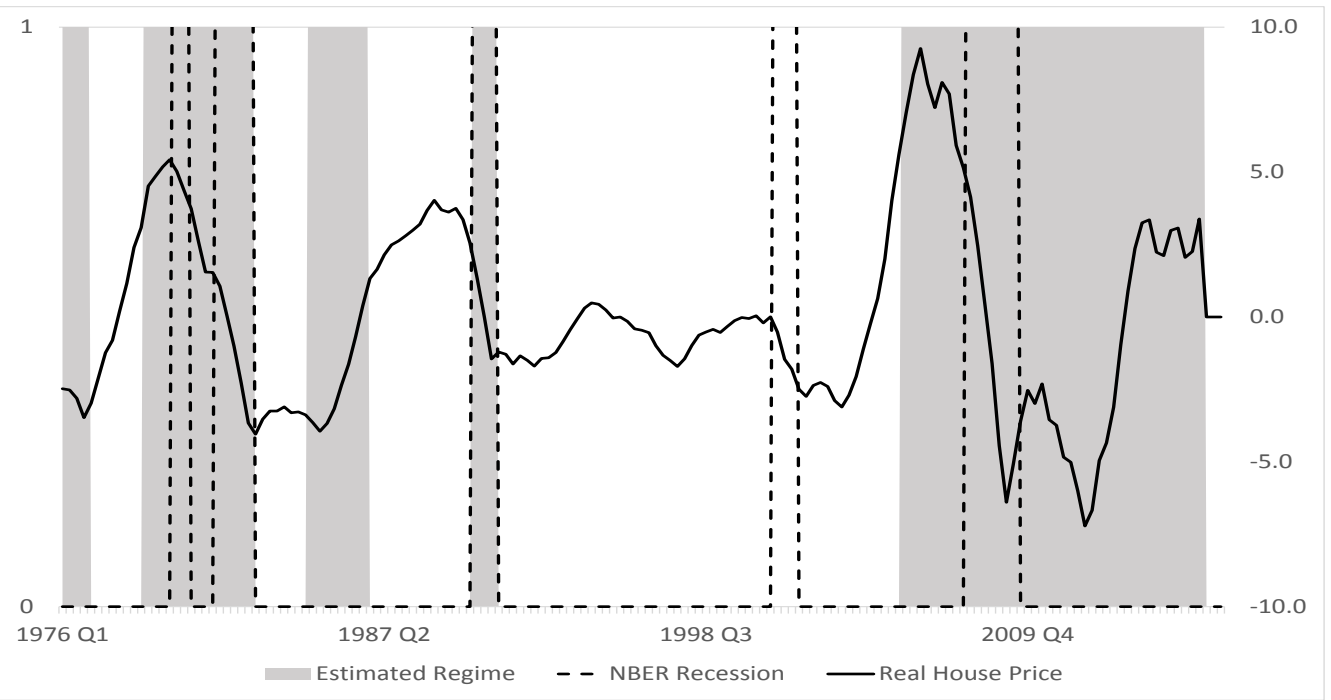

Notes: A black solid line is the HP-filtered real house price.

Figure 2.4: Regime Indicator

as the mortgage rate. The rent to house price ratio is computed as the ratio between the housing CPI component (BLS) and the nominal Shiller house price index. We take the log transformation for all variables, except for CPI inflation, Treasury bill rate, and mortgage rate, and linearly detrend them.

\subsubsection{House Price Uncertainty Shocks and Economic Activ- ity}

Figure 2.4 shows that the regime- 2 obtained by the MS-VAR model is associated with the NBER recessionary periods. This implies that the regime-1 is likely to correspond to the stable and expansionary periods. Figure 2.5 and 2.6 plot the median of impulse responses in each regime to a two-standard-deviation house price uncertainty shock with a $68 \%$ confidence interval. Figure 2.6 shows that the house price uncertainty shock has negative effects on macroeconomic aggregates in regime 2 . It initially reduces consumption by around $0.2 \%$ via a precautionary savings motive. Residential investment decreases by nearly $1.5 \%$. This can be explained by real-options channels, implying that the house price uncertainty shock has a negative effect on the real- 
options value of a new residential investment project. Both of the responses are quite significant at the medium run with their peak responses occurring after about 10 quarters. The peak decline in residential investment is around five times as large as the peak decline in consumption. Inflation falls initially following the shock, as documented in other studies, though it is not statistically significant. The monetary policy rate reacts to the downturn in economic activities and inflation by lowering rates. However, mortgage rates rise, increasing the mortgage spread.

The shock in regime 2 generates positive co-movement between aggregate macroeconomic variables and housing market-related variables. Real house prices and mortgage debt decrease by $1.0 \%$ and $3.0 \%$ at the peak, respectively. These results imply that the financial and debt risk effect would be stronger than the hedging effect so that the demand for housing falls, requiring a lower house price. Since mortgage debt is contemporaneously related to housing prices via collateral constraints, the fall in house prices, driven by the financial and debt risk effect, leads to a fall in mortgage debt with a high persistence level. Finally, rent-to-price ratio increases and reverts back to the original level. This suggests that the shock could lead to a drop in renting houses to a smaller extent than the magnitude of the drop in demand for housing.

However, in regime 1, which contains the housing boom periods, house prices, residential investment, and mortgage debt increase in response to the house price uncertainty shock. These results are the notable differences between the regime-1 and the regime-2. Households in the expansionary regime are more likely to have a strong desire for more housing consumption to hedge against future housing consumption risks. A drop in rent to price ratio reflects these facts. The increase in house prices leads to a rise in mortgage debt. The magnitude of the decline in consumption is slightly larger in regime 2 (in Figure 2.6) than that in regime 1 (in Figure 2.6). This possibly reflects the fact that the recent financial crisis, with massive uncertainty about future economic conditions, is contained in this regime so that the precau- 

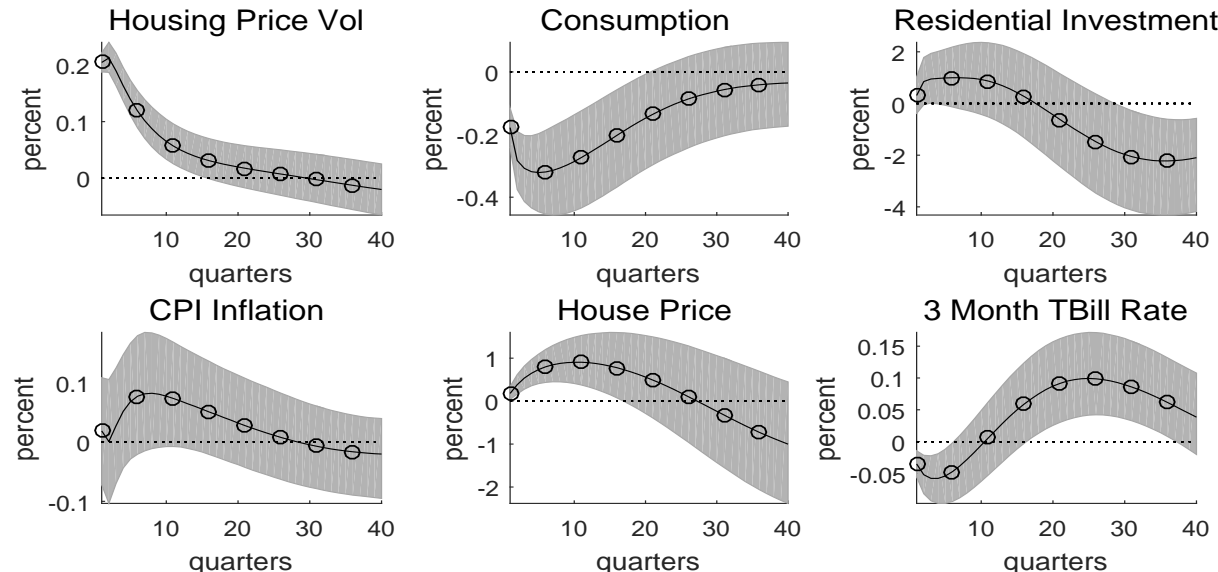

House Price
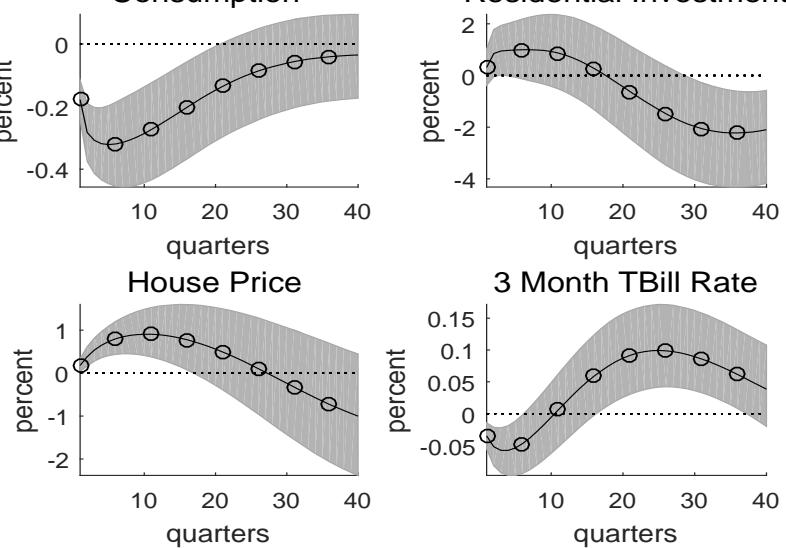

3 Month TBill Rate
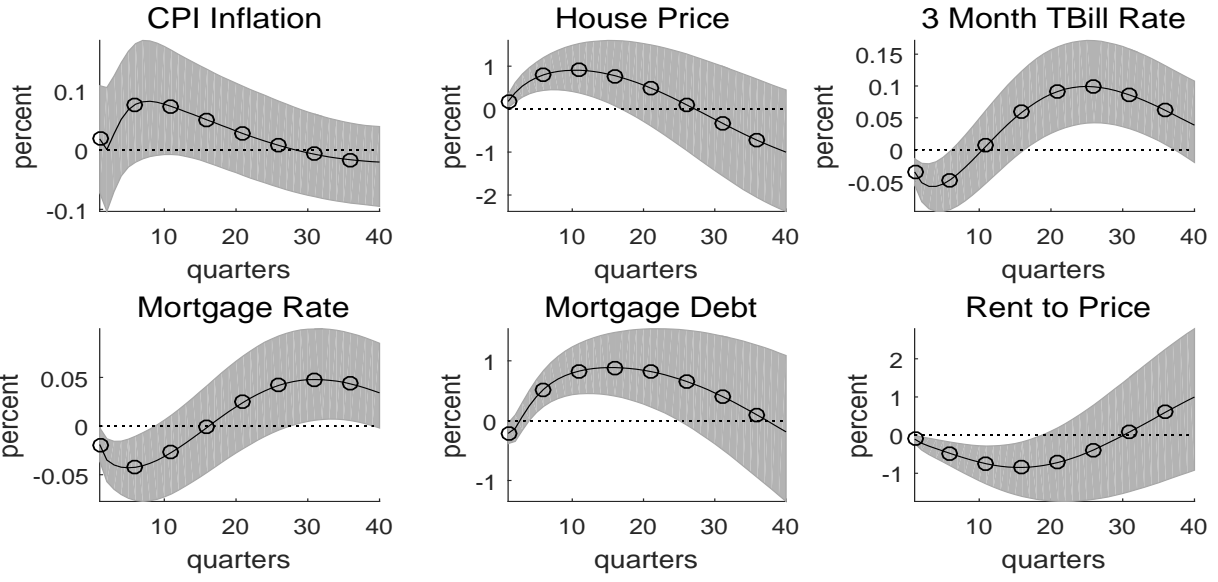

Figure 2.5: Responses to House Price Uncertainty Shock in Regime1 (Expansionary)
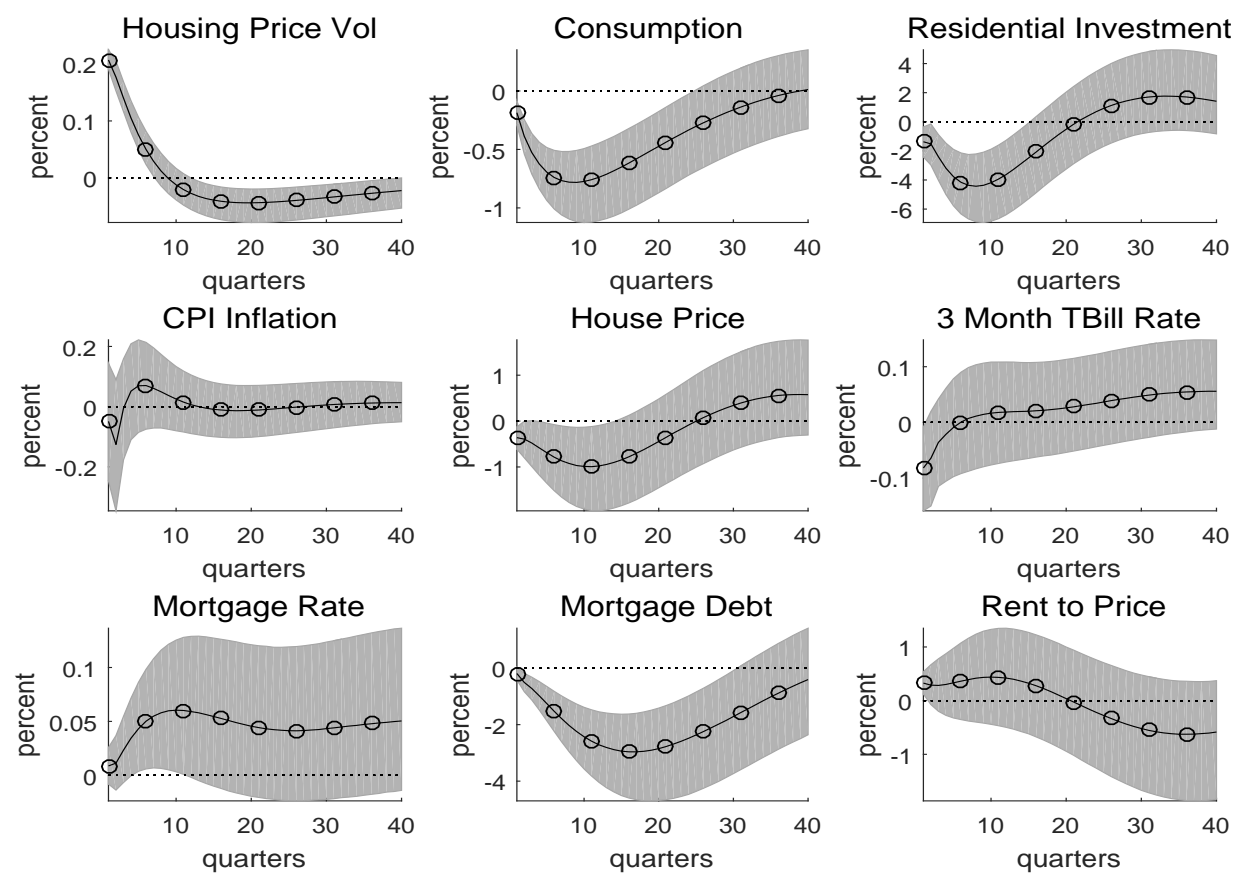

Figure 2.6: Responses to House Price Uncertainty Shock in Regime2 (Recessionary) 
Table 2.2 Forecast Error Variance Decomposition

\begin{tabular}{|c|c|c|c|c|c|c|c|c|}
\hline \multicolumn{9}{|c|}{ Regime 1} \\
\hline Horizon & Consumption & $\begin{array}{l}\text { Residential } \\
\text { Investment }\end{array}$ & CPI & $\begin{array}{l}\text { Housing } \\
\text { Price }\end{array}$ & $\begin{array}{l}3 \text { Month } \\
\text { TBill Rate }\end{array}$ & $\begin{array}{c}\text { Mortgage } \\
\text { Rate }\end{array}$ & $\begin{array}{c}\text { Mortgage } \\
\text { Debt }\end{array}$ & Rent/Price \\
\hline $1 \mathrm{Q}$ & 8.62 & 0.61 & 0.53 & 1.96 & 3.42 & 1.75 & 1.78 & 1.39 \\
\hline $10 \mathrm{Q}$ & 27.16 & 4.18 & 4.46 & 14.60 & 6.12 & 8.60 & 12.77 & 9.44 \\
\hline $20 \mathrm{Q}$ & 28.98 & 5.88 & 6.95 & 10.85 & 11.48 & 9.98 & 18.64 & 8.89 \\
\hline $30 \mathrm{Q}$ & 28.06 & 9.73 & 8.35 & 8.83 & 18.32 & 14.71 & 13.72 & 6.76 \\
\hline $40 \mathrm{Q}$ & 26.72 & 13.99 & 9.08 & 9.92 & 19.24 & 16.85 & 10.81 & 7.64 \\
\hline \multicolumn{9}{|c|}{ Regime 2} \\
\hline Horizon & Consumption & $\begin{array}{l}\text { Residential } \\
\text { Investment }\end{array}$ & CPI & $\begin{array}{l}\text { Housing } \\
\text { Price }\end{array}$ & $\begin{array}{l}3 \text { Month } \\
\text { TBill Rate }\end{array}$ & $\begin{array}{c}\text { Mortgage } \\
\text { Rate }\end{array}$ & $\begin{array}{c}\text { Mortgage } \\
\text { Debt }\end{array}$ & Rent/Price \\
\hline $1 \mathrm{Q}$ & 4.28 & 2.28 & 0.61 & 3.04 & 1.58 & 0.99 & 1.17 & 3.30 \\
\hline $10 \mathrm{Q}$ & 31.37 & 14.34 & 3.78 & 5.69 & 3.54 & 4.86 & 19.28 & 2.91 \\
\hline $20 \mathrm{Q}$ & 33.76 & 14.89 & 4.89 & 8.53 & 5.18 & 6.63 & 25.43 & 5.02 \\
\hline $30 \mathrm{Q}$ & 31.86 & 15.07 & 5.65 & 10.03 & 6.95 & 8.45 & 25.38 & 7.11 \\
\hline $40 \mathrm{Q}$ & 30.32 & 15.75 & 6.19 & 11.43 & 8.69 & 10.59 & 24.29 & 8.65 \\
\hline
\end{tabular}

tionary motive in regime 2 is much more severe than that in regime 1. Moreover, the decline in house prices could lead the response of aggregate consumption to be amplified by the wealth effects and collateral constraints channel.

Table 2.2 presents the fraction of forecast error variance explained by the house price uncertainty shock for the endogenous variables at each regime. The initial contribution of the uncertainty shock is small, with the maximum share being $8.62 \%$ and $4.28 \%$ for consumption and the minimum share being $0.53 \%$ and $0.61 \%$ for CPI inflation in regime 1 and regime 2, respectively. However, as the forecast horizon increases, the house price uncertainty shock tends to account for a larger fraction of macroeconomic variables. The shock in regime 2 is more likely to explain fluctuations of consumption, residential investment, housing price, and mortgage debt at longer horizons than the shock in regime 1 , though the shock in regime 1 accounts for a larger fraction of housing price at shorter horizons than the shock in regime 2 . This result implies that the house price uncertainty shock plays a more significant role in explaining economic fluctuations in recessionary periods than in stable and expansionary periods at longer horizons. At a 40 quarter horizon, the shock explains $27 \%$ (30\%) of consumption variation, $14 \%$ (16\%) of residential investment variation, 
$10 \%(11 \%)$ of housing price variation, and $11 \%$ (24\%) of mortgage debt variation in regime 1 (in regime 2). The significant rise in the contribution to consumption and mortgage debt at longer horizons might be driven by the wealth effects and collateral constraints channel.

\subsection{Theoretical DSGE Model with a Housing Mar- ket}

The MS-VAR model considered in Section 4 serves as a useful benchmark model by empirically capturing the asymmetric effects of house price uncertainty shocks on macroeconomic aggregates. However, it does not guarantee the one-to-one mapping with the structural DSGE model due to nonlinear terms in the third-order approximation with stochastic volatility. ${ }^{7}$ The MS-VAR model can be thought of as a reducedform version of the Markov-switching DSGE model. In this study, however, we focus on linking potential sources of regime-dependent house price uncertainty shocks to two different types of structural volatility shocks (supply-side volatility shocks and demand-side volatility shocks) in the non-regime-dependent DSGE model. Since the house price uncertainty shocks are associated with second-moment shocks in the DSGE model, we assume that the house price uncertainty shocks are the combination of structural volatility shocks in the DSGE model, and their relationship is likely to change over time, depending on the phase of business cycle. Although the recursive identification scheme allows us to identify the house price uncertainty shocks, it is hard to identify each structural volatility shock that is a possible source of the house price uncertainty shocks. In this section, following the spirit of sign restrictions as in Mumtaz and Theodoridis (2015), we find major sources of the house price uncertainty shocks based on model-implied impulse responses to structural volatility shocks.

\footnotetext{
${ }^{7}$ To take volatility shocks into account in the DSGE model, it is required to approximate the optimized behaviors up to third-order (Fernández-Villaverde et al., 2011, 2015).
} 
This section describes a DSGE model for theoretical analysis. The model is based on Iacoviello and Neri (2010), but it allows for a rental housing market based on MoraSanguinetti and Rubio (2014); Gazzani (2016); Sun and Tsang (2017) and multiple stochastic volatility shocks. Incorporating a rental housing market into the model leads us to better understand a transmission mechanism of the housing consumption hedging effect driven by house price uncertainty. The stochastic volatility process to each shock in the model plays a significant role in analyzing the heterogeneous effects of uncertainty shocks on the housing market and finding major sources of the house price uncertainty shocks.

\subsubsection{Model}

In this economy, there are two types of households: lenders (patient households) and borrowers (impatient households). They mainly differ in terms of the discount factor. Both of them consume nonhousing consumption goods and housing services and supply labors. Lenders rent capital to firms, lend to firms and borrowers, and rent out a part of the housing stock to borrowers. They benefit from a flow of housing services only by owning the housing stock. On the other hand, borrowers benefit from consuming housing services by owning the housing stock and renting the housing stock from lenders. They can borrow against the value of the housing stock from lenders.

A representative household of lenders maximizes the discounted sum of expected utility:

$$
E_{0} \sum_{t=0}^{\infty}\left(\beta^{L}\right)^{t} z_{t}\left\{\Gamma_{c}^{L} \ln \left(c_{t}^{L}-\varepsilon^{L} c_{t-1}^{L}\right)+j_{t} \ln h_{t-1}^{L}-\frac{\tau_{t}}{1+\eta^{L}}\left(\left(n_{c, t}^{L}\right)^{1+\xi^{L}}+\left(n_{h, t}^{L}\right)^{1+\xi^{L}}\right)^{\frac{1+\eta^{L}}{1+\xi^{L}}}\right\}
$$

where $c_{t}^{L}$ is nonhousing consumption, $h_{t}^{L}$ is the housing stock, and $n_{c, t}^{L}$ and $n_{h, t}^{L}$ are hours worked in the consumption and housing sector. We assume that the housing stock purchased at the end of $t-1$ produces housing services at the beginning of $t$. 
$z_{t}$ and $\tau_{t}$ are shocks to inter-temporal preferences and to labor supply, respectively, and $j_{t}$ captures shocks to housing preference. $\varepsilon^{L}$ measures habits in consumption, the parameter $\eta^{L}>0$ is the inverse of the Frisch labor supply elasticity, $\xi^{L}$ measures imperfect substitutability between work hours in the two sectors, and the scaling factor $\Gamma_{c}^{L}$ ensures a simple form of marginal utility of consumption in the steady state $\left(1 / c^{L}\right)$. All the shocks in the utility function follow $\mathrm{AR}(1)$ processes with normally distributed shocks and heteroskedastic standard deviations $\sigma_{z, t}, \sigma_{j, t}$, and $\sigma_{\tau, t}$. Lenders make optimal decisions based on the following budget constraint:

$$
\begin{aligned}
& c_{t}^{L}+\frac{k_{c, t}}{A_{k, t}}+k_{h, t}+k_{b, t}+q_{t}\left[h_{t}^{L}-\left(1-\delta_{h}\right) h_{t-1}^{L}+h_{t}^{r}-\left(1-\delta_{h}\right) h_{t-1}^{r}\right]+p_{l, t} l_{t}-b_{t}^{L} \\
& =\frac{w_{c, t}^{L} n_{c, t}^{L}}{X_{w c, t}^{L}}+\frac{w_{h, t}^{L} n_{h, t}^{L}}{X_{w h, t}^{L}}+\left(R_{c, t} z_{c, t}+\frac{1-\delta_{k c}}{A_{k, t}}\right) k_{c, t-1}+\left(R_{h, t} z_{h, t}+1-\delta_{k h}\right) k_{h, t-1} \\
& +p_{b, t} k_{b, t}-\frac{R_{t-1} b_{t-1}^{L}}{\pi_{t}}+\left(p_{l, t}+R_{l, t}\right) l_{t-1}+q_{t}^{r} h_{t-1}^{r}+D i v_{t}^{L}-\phi_{t}-\frac{a\left(z_{c, t}\right) k_{c, t-1}}{A_{k, t}}-a\left(z_{h, t}\right),
\end{aligned}
$$

where $k_{c, t}$ and $k_{h, t}$ are capital in the consumption and housing sector with real rental rates $R_{c, t}$ and $R_{h, t}$, respectively. The term $A_{k, t}$ are investment-specific technology shocks. $k_{b, t}$ are intermediate inputs priced at $p_{b, t}, h_{t}^{r}$ is the housing stock for rent, $q_{t}$ is the price of housing in real terms, $q_{t}^{r}$ captures real rental rates for one unit of housing, $l_{t}$ is land priced at $p_{l, t}$ with real rental rates of $R_{l, t}, b_{t}^{L}$ is the amount of lending with a riskless nominal return $R_{t}, w_{c, t}^{L}$ and $w_{h, t}^{L}$ are real wages paid in the two sectors of production, $X_{w c, t}^{L}$ and $X_{w h, t}^{L}$ are the markups which denote wages paid by the wholesale firms over wages paid to the households, $z_{c, t}$ and $z_{h, t}$ are capital utilization in the consumption and housing sector, and $\pi_{t}$ is gross inflation $\left(=\frac{P_{t}}{P_{t-1}}\right)$. $\delta_{h}$ is the depreciation rate of house, $\delta_{k c}$ and $\delta_{k h}$ are the depreciation rates of capital in the consumption and housing sector. Div $v_{t}^{L}$ are dividends paid from final good retailers 
and from labor unions. ${ }^{8}$ It implies that uncertainty shocks could be amplified through the markup of final goods over wholesale goods and the markups between the wage costs for the wholesale firms and households' wages. $\phi_{t}$ is capital adjustment costs and $a\left(z_{c, t}\right)$ and $a\left(z_{h, t}\right)$ are adjustment costs on capacity utilization (see the details of $\phi_{t}$ and $a(\cdot)$ in Appendix B.4).

The optimal conditions with respect to $h_{t}^{L}$ and $h_{t}^{r}$ are as follows:

$$
\begin{gathered}
u_{c^{L}, t}^{L} q_{t}=\beta^{L} E_{t}\left[\frac{j_{t+1} z_{t+1}}{h_{t}^{L}}\right]+\beta^{L} E_{t}\left[u_{c^{L}, t+1}^{L} q_{t+1}(1-\delta)\right], \\
u_{c^{L}, t}^{L} q_{t}=\beta^{L} E_{t}\left[u_{c^{L}, t+1}^{L} q_{t+1}^{r}\right]+\beta^{L} E_{t}\left[u_{c^{L}, t+1}^{L} q_{t+1}(1-\delta)\right] .
\end{gathered}
$$

By combining (2.7) and (2.8), we obtain

$$
E_{t}\left[u_{c^{L}, t+1}^{L} q_{t+1}^{r}\right]=\beta^{L} E_{t}\left[\frac{j_{t+1} z_{t+1}}{h_{t}^{L}}\right]
$$

If the equation (2.9) holds, then households are indifferent between consuming housing services and renting a part of the housing stock to borrowers. By iterating (2.7) and (2.8), we also obtain

$$
q_{t}=E_{t}\left[\sum_{i=1}^{\infty}\left(\beta^{L}\right)^{i} q_{t+i}^{r}\right]=E_{t}\left[\sum_{i=1}^{\infty}\left(\beta^{L}\right)^{i} \frac{j_{t+i}}{h_{t}^{L}}\right]
$$

The equation (2.10) implies that real house prices are the infinite sum of discounted future real rental rates (or housing preference).

In a similar way, borrowers maximize the following discounted sum of expected

${ }^{8}$ Div $_{t}^{L}$ are given as follows:

$$
D i v_{t}^{L}=\left(1-\frac{1}{X_{t}}\right) Y_{t}+\left(1-\frac{1}{X_{w c, t}^{L}}\right) w_{c, t}^{L} n_{c, t}^{L}+\left(1-\frac{1}{X_{w h, t}^{L}}\right) w_{h, t}^{L} n_{h, t}^{L}
$$

where $X_{t}$ denotes the markup of final goods over wholesale goods. $X_{w c, t}^{L}$ and $X_{w h, t}^{L}$ are the markups between the wage costs for the wholesale firm and households' wages. 
utility:

$$
E_{0} \sum_{t=0}^{\infty}\left(\beta^{B}\right)^{t} z_{t}\left\{\Gamma_{c}^{B} \ln \left(c_{t}^{B}-\varepsilon^{B} c_{t-1}^{B}\right)+j_{t} \ln \hat{h}_{t-1}^{B}-\frac{\tau_{t}}{1+\eta^{B}}\left(\left(n_{c, t}^{B}\right)^{1+\xi^{B}}+\left(n_{h, t}^{B}\right)^{1+\xi^{B}}\right)^{\frac{1+\eta^{B}}{1+\xi^{B}}}\right\}
$$

where housing services $\hat{h}_{t}^{B}$ come from the housing stock $h_{t}^{B}$ borrowers own and the housing stock $h_{t}^{r}$ they rent from lenders. They are aggregated through the following CES function:

$$
\hat{h}_{t}^{B}=\left[\kappa\left(h_{t}^{B}\right)^{\xi_{h}-1}+(1-\kappa)\left(h_{t}^{r}\right)^{\xi_{h}-1}\right]^{\frac{1}{\xi_{h}-1}} .
$$

Borrowers maximize their utility subject to the following budget constraint:

$$
c_{t}^{B}+q_{t}\left[h_{t}^{B}-\left(1-\delta_{h}\right) h_{t-1}^{B}\right]+q_{t}^{r} h_{t-1}^{r}-b_{t}^{B}=\frac{w_{c, t}^{B} n_{c, t}^{B}}{X_{w c, t}^{B}}+\frac{w_{h, t}^{B} n_{h, t}^{B}}{X_{w h, t}^{B}}-\frac{R_{t-1} b_{t-1}^{B}}{\pi_{t}}+D i v_{t}^{B},
$$

where $D i v_{t}^{B}$ are dividends from labor unions. Borrowers are allowed to borrow up to the expected present value of their houses times the loan-to-value (LTV) ratio

$$
b_{t}^{B} \leq m E_{t}\left[\frac{q_{t+1} \pi_{t+1} h_{t}^{B}}{R_{t}}\right]
$$

where $m$ is the LTV ratio.

To better understand the mechanism of the financial risk effect, the hedging effect, and the debt risk effect of house price uncertainty, we describe the optimal decisions on the housing stock for owning and renting. We will describe the details of the mechanism in subsection 2.6.4.

$$
\begin{aligned}
u_{c^{B}, t}^{B} q_{t}^{r}= & \beta^{B} E_{t}\left[\frac{j_{t+1} z_{t+1}}{h_{t}^{B}}\left[\kappa\left(h_{t}^{B}\right)^{\xi_{h}-1}+(1-\kappa)\left(h_{t}^{r}\right)^{\xi_{h}-1}\right]^{\frac{2-\xi_{h}}{\xi_{h}-1}}(1-\kappa)\left(h_{t}^{r}\right)^{\xi_{h}-2}\right], \\
u_{c^{B}, t}^{B} q_{t} & =\beta^{B} E_{t}\left[\frac{j_{t+1} z_{t+1}}{h_{t}^{B}}\left[\kappa\left(h_{t}^{B}\right)^{\xi_{h}-1}+(1-\kappa)\left(h_{t}^{r}\right)^{\xi_{h}-1}\right]^{\frac{2-\xi_{h}}{\xi_{h}-1}} \kappa\left(h_{t}^{B}\right)^{\xi_{h}-2}\right] \\
& +\beta^{B} E_{t}\left[u_{c^{B}, t+1}^{B} q_{t+1}(1-\delta)\right]+E_{t}\left[\frac{\lambda_{t} m q_{t+1} \pi_{t+1}}{R_{t}}\right] .
\end{aligned}
$$


Wholesale firms that produce wholesale goods $(Y)$ and new houses $(I H)$ solve the following profit maximization problem:

$$
\max \frac{Y_{t}}{X_{t}}+q_{t} I H_{t}-\left(\sum_{i=c, h} w_{i, t}^{L} n_{i, t}^{L}+\sum_{i=c, h} w_{i, t}^{B} n_{i, t}^{B}+\sum_{i=c, h} R_{i, t} z_{i, t} k_{i, t-1}+R_{l, t} l_{t-1}+p_{b, t} k_{b, t}\right),
$$

where $X_{t}$ is the markup between final goods and wholesale goods $\left(\frac{P_{t}}{P_{t}^{W}}\right)$. Both the consumption and housing sector face Cobb-Douglas production functions. The nonhousing consumption sector uses capital $\left(k_{c}\right)$ and labor supplied by lenders $\left(n_{c}^{L}\right)$ and borrowers $\left(n_{c}^{B}\right)$ to produce wholesale goods. The housing sector uses capital $\left(k_{h}\right)$, land $(l)$, intermediate inputs $\left(k_{b}\right)$ and labor $\left(n_{h}^{L}\right.$ and $\left.n_{h}^{B}\right)$ as inputs of production.

$$
\begin{gathered}
Y_{t}=\left(A_{c, t}\left(\left(n_{c, t}^{L}\right)^{\alpha}\left(n_{c, t}^{B}\right)^{1-\alpha}\right)\right)^{1-\mu_{c}}\left(z_{c, t} k_{c, t-1}\right)^{\mu_{c}}, \\
I H_{t}=\left(A_{h, t}\left(\left(n_{h, t}^{L}\right)^{\alpha}\left(n_{h, t}^{B}\right)^{1-\alpha}\right)\right)^{1-\mu_{h}-\mu_{b}-\mu_{l}}\left(z_{h, t} k_{h, t-1}\right)^{\mu_{c}} k_{b, t}^{\mu_{b}} l_{t-1}^{\mu_{l}},
\end{gathered}
$$

where $A_{c, t}$ and $A_{h, t}$ are productivity in the consumption and housing sector. The parameter $\mu_{c}$ is the share of capital in the production function for the nonhousing sector, the parameters, $\mu_{h}, \mu_{b}, \mu_{l}$ are, respectively, the shares of capital, intermediate inputs, and land in the production function for the housing sector, $\alpha$ measures the labor income share of lenders and $1-\alpha$ of borrowers.

A continuum of retailers of mass 1 , indexed by $z$, purchase wholesale goods $Y_{t}$ and differentiate them by adding a unique feature to the product. They sell the differentiated product $Y_{t}(z)$ at the price $P_{t}(z)$. Monopolistic competition occurs at the retail level. Prices are adjusted with probability $1-\theta_{\pi}$ every period based on a Calvo scheme, while a fraction $\theta_{\pi}$ indexes prices to lagged inflation at $\iota_{\pi}$. Retailers take this constraint into account when maximizing the following expected profits with 
respect to $P_{t}(z)$

$$
\sum_{k=0}^{\infty}\left(\beta^{L} \theta_{\pi}\right)^{k} E_{t}\left\{\frac{\lambda_{t+k}^{L}}{\lambda_{t}^{L}}\left\{\frac{\pi_{t-1, t+k-1}^{\iota_{\pi}} P_{t}(z)}{P_{t+k}} Y_{t+k}(z)-\frac{1}{X_{t+k}} Y_{t+k}(z)\right\}\right\}
$$

where $\lambda_{t}^{L}$ is the marginal utility of consumption for lenders and $Y_{t+k}(z)$ is an individual demand $\left(=\left(\frac{\pi_{t+k-1}^{\iota \pi} P_{t}(z)}{P_{t+k}}\right)^{-\varepsilon_{p}} Y_{t+k}\right)$ that the retailers face. ${ }^{9}$ Under the assumption that the retailers face the same optimization problem, all firms choose the following price level $P_{t}^{*}$.

$$
\begin{aligned}
P_{t}^{*} & =\frac{\varepsilon_{p}}{\varepsilon_{p}-1} \frac{X_{1 t}}{X_{2 t}}, \\
X_{1 t} & =\lambda_{t}^{L} \frac{1}{X_{t}} P_{t}^{\varepsilon_{p}} Y_{t}+\beta \theta \pi_{t}^{-\iota_{\pi} \varepsilon_{p}} E_{t} X_{1 t+1}, \\
X_{2 t} & =\lambda_{t}^{L} P_{t}^{\varepsilon_{p}-1} Y_{t}+\beta \theta \pi_{t}^{\iota_{\pi}\left(1-\varepsilon_{p}\right)} E_{t} X_{2 t+1} .
\end{aligned}
$$

To express the optimal pricing conditions in terms of inflation rates, we divide $P_{t}^{*}$ by $P_{t-1}$.

$$
\begin{aligned}
\pi_{t}^{*} & =\frac{P_{t}^{*}}{P_{t-1}}=\frac{\varepsilon_{p}}{\varepsilon_{p}-1} \pi_{t} \frac{x_{1 t}}{x_{2 t}}, \\
x_{1 t} & =\lambda_{t}^{L} \frac{1}{X_{t}} Y_{t}+\beta \theta \pi_{t}^{-\iota_{\pi} \varepsilon_{p}} E_{t} \pi_{t+1}^{\varepsilon_{p}} x_{1 t+1}, \\
x_{2 t} & =\lambda_{t}^{L} Y_{t}+\beta \theta \pi_{t}^{\iota_{\pi}\left(1-\varepsilon_{p}\right)} E_{t} \pi_{t+1}^{\varepsilon_{p}-1} x_{2 t+1} .
\end{aligned}
$$

As a fraction $\theta_{\pi}$ of prices is unchanged, the gross inflation of the aggregate price is

${ }^{9}$ The demand function is derived from the assumption that the differentiated goods are aggregated by the CES function:

$$
Y_{t}=\left(\int_{0}^{1} Y_{t}(z)^{\left(\varepsilon_{p}-1\right) / \varepsilon_{p}} d z\right)^{\varepsilon_{p} /\left(\varepsilon_{p}-1\right)} .
$$

The aggregate price index is given by

$$
P_{t}=\left(\int_{0}^{1} P_{t}(z)^{\left(1-\varepsilon_{p}\right)} d z\right)^{1 /\left(1-\varepsilon_{p}\right)} .
$$


given by

$$
\pi_{t}^{1-\varepsilon_{p}}=\left(1-\theta_{\pi}\right) \pi_{t}^{*\left(1-\varepsilon_{p}\right)}+\theta_{\pi} \pi_{t-1}^{\iota_{\pi}\left(1-\varepsilon_{p}\right)} .
$$

In a similar way, wages are set in a monopolistic way based on a Calvo scheme with a given probability $1-\theta_{w, i}$ every period. Households supply labor to the labor unions who differentiate labor services. The unions sell the differentiated labor $N_{i, t}^{j}(z)$ to wholesale labor packers at $w_{i, t}^{j}(z)$. The packers aggregate labor services based on the CES aggregates and provide labor services to wholesale firms.

$$
\sum_{k=0}^{\infty}\left(\beta^{j} \theta_{w, i}\right)^{k} E_{t}\left\{\frac{\lambda_{t+k}^{j}}{\lambda_{t}^{j}}\left\{\pi_{t-1, t+k-1}^{\iota_{w, i}} \pi_{t, t+k}^{-1} w_{i, t}^{j}(z) N_{i, t+k}^{j}(z)-\frac{w_{t+k}}{X_{i, t+k}^{j}} N_{i, t+k}^{j}(z)\right\}\right\},
$$

where $N_{i, t+k}^{j}(z)$ is the demand function $\left(=\left(\frac{\pi_{t-1, t+k-1}^{\iota_{w}, i} \pi_{t, t+k}^{-1} w_{i, t}^{j}(z)}{w_{i, t+k}^{j}}\right)^{-\varepsilon_{i, w}^{j}} n_{i, t+k}^{j}\right)$ that the labor union faces, $X_{i, t+k}^{j}$ is the markup, and $\lambda_{t}^{j}$ is the marginal utility of consumption. The sticky wage leads to the following optimal real wage:

$$
\begin{aligned}
& w_{i, t}^{* j}=\frac{\varepsilon_{i, w}^{j}}{\varepsilon_{i, w}^{j}-1} \frac{f_{i, 1 t}^{j}}{f_{i, 2 t}^{j}}, \\
& f_{i, 1 t}^{j}=\lambda_{t}^{j} \frac{\left(w_{i, t}^{j}\right)^{1+\varepsilon_{i, w}^{j}}}{X_{i, t}^{j}} n_{i, t}^{j}+\beta^{j} \theta_{w, i} \pi_{t}^{-\iota_{w, i} \varepsilon_{i, w}^{j}} E_{t} \pi_{t+1}^{\varepsilon_{i, w}^{j}} f_{i, 1 t+1}^{j}, \\
& f_{i, 2 t}^{j}=\lambda_{t}^{j}\left(w_{i, t}^{j}\right)^{\varepsilon_{i, w}^{j}} n_{i, t}^{j}+\beta^{j} \theta_{w, i} \pi_{t}^{\iota_{w, i}\left(1-\varepsilon_{i, w}^{j}\right)} E_{t} \pi_{t+1}^{\varepsilon_{i, w}^{j}-1} f_{i, 2 t+1}^{j} .
\end{aligned}
$$

where $i=$ consumption sector (C) or housing sector (H) and $j=$ Borrowers (B) or lenders $(\mathrm{L})$. As a fraction $\theta_{w, i}$ of wages is unchanged, the aggregate real wage is given by

$$
\left(w_{i, t}^{j}\right)^{1-\varepsilon_{i, w}}=\left(1-\theta_{w, i}\right)\left(w_{i, t}^{* j}\right)^{\left(1-\varepsilon_{i, w}^{j}\right)}+\theta_{w, i} \pi_{t-1}^{\iota_{w, i}\left(1-\varepsilon_{i, w}^{j}\right)} \pi_{t}^{\varepsilon_{i, w}^{j}-1}\left(w_{i, t-1}^{j}\right)^{\left(1-\varepsilon_{i, w}^{j}\right)} .
$$


Monetary authority follows a Taylor-type rule:

$$
R_{t}=R_{t-1}^{r_{R}} \pi_{t}^{\left(1-r_{R}\right) r_{\pi}}\left(\frac{G D P_{t}}{G D P_{t-1}}\right)^{\left(1-r_{R}\right) r_{Y}} \overline{r r}^{1-r_{R}} \frac{\sigma_{R, t} u_{R, t}}{A_{s, t}} \quad \text { where } u_{R, t} \sim N(0,1)
$$

where $\overline{r r}$ is the steady state real interest-rate, $r_{R}, r_{\pi}, r_{Y}$ are the responses of the interest rate to changes in the lagged interest rate, inflation and GDP growth, respectively, the inflation target shock $\left(A_{s, t}\right)$ follows $\mathrm{AR}(1)$ process with stochastic volatility $\sigma_{s, t}$, and the shock to the monetary policy $u_{R, t}$ is assumed to have time-varying volatility $\sigma_{R, t}$.

In the equilibrium,

$$
\begin{gathered}
C_{t}+I K_{c, t} / A_{k, t}+I K_{h, t}+k_{b, t}=Y_{t}-\phi_{t}, \\
H_{t}-\left(1-\delta_{h}\right) H_{t-1}=I H_{t}, \\
b_{t}^{L}+b_{t}^{B}=0, \\
l_{t}=1,
\end{gathered}
$$

where

$$
\begin{gathered}
I K_{c, t}=k_{c, t}-\left(1-\delta_{k c}\right) k_{c, t-1}, \\
I K_{h, t}=\frac{k_{h, t}-\left(1-\delta_{k h}\right) k_{h, t-1}}{A_{k, t}} .
\end{gathered}
$$

Finally, productivity in the consumption $\left(A_{c, t}\right)$, investment $\left(A_{k, t}\right)$, and housing sector $\left(A_{h, t}\right)$ follows:

$$
\ln \left(A_{i, t}\right)=\rho_{A, i} \ln \left(A_{i, t-1}\right)+\exp \left(\sigma_{i, t}\right) u_{i, t} \quad \text { where } u_{i, t} \sim N(0,1)
$$

where $i=\{c, k, h\}$ and $u_{i, t}$ is the innovation. All of the stochastic volatility processes 
Table 2.3 Calibrated Parameters

\begin{tabular}{c|l|c}
\hline \multicolumn{2}{l|}{ parameter } & value \\
\hline$\beta$ & discount factor, lenders & 0.9925 \\
$\beta$ & discount factor, borrowers & 0.97 \\
$j$ & weight on housing & 0.12 \\
$\mu_{c}$ & capital share in the goods production function & 0.35 \\
$\mu_{h}$ & capital share in the housing production function & 0.1 \\
$\mu_{l}$ & land share & 0.1 \\
$\mu_{b}$ & intermediate goods share & 0.1 \\
$\delta_{h}$ & depreciation rates for housing & 0.01 \\
$\delta_{k} c$ & depreciation rates for capital in the consumption & 0.025 \\
$\delta_{k} h$ & depreciation rates for capital in the housing & 0.03 \\
$\varepsilon_{p}, \varepsilon_{c, w}^{L}, \varepsilon_{h, w}^{L}, \varepsilon_{c, w}^{B}, \varepsilon_{h, w}^{B}$ & steady-state gross price and wage markups & 6 \\
$m$ & loan to value ratio (LTV) & 0.85 \\
$\rho_{s}$ & correlation of the inflation objective shock & 0.975 \\
$\rho_{s}$ & correlation of the inflation objective uncertainty shock & 0.956 \\
$\rho_{s}$ & std. inflation objective uncertainty shock & 0.258 \\
$\kappa$ & preference for owning home & 0.6 \\
\hline
\end{tabular}

follow:

$$
\sigma_{j, t}=\left(1-\rho_{\sigma_{j}}\right) \sigma_{j}+\rho_{\sigma_{j}} \sigma_{j, t-1}+\eta_{j} v_{j, t} \quad \text { where } v_{j, t} \sim N(0,1),
$$

where $j \in\{c, h, j, k, \tau, z, s, R\}, \sigma_{j, t}$ is the $j$-th stochastic volatility, $\sigma_{j}$ is the unconditional mean of $\sigma_{j, t}, \rho_{\sigma_{j}}$ is the level of persistence, and $\eta_{j}$ is the standard deviation of the volatility shock $v_{j, t}$. All of the equilibrium conditions are given in Appendix B.4.

\subsubsection{Solution and Calibration}

We solve the model based on a third-order approximation that allows time-varying volatility to be an independent component of the decision rules. This approximation is crucial when we investigate the direct effects of uncertainty shocks on economic activity. However, higher-order approximations that consist of polynomials of state variables have multiple steady states and can yield unbounded solutions. As explained by Kim et al. (2008), simulated sample paths of the approximated policy function could explode when the accumulation of higher-order effects is significantly large, generating unstable steady states. To solve this problem, they suggest applying a pruning procedure to a second-order approximation that eliminates terms of higherorder effects than the approximation order. Andreasen et al. (2017) extend this logic to any order and provide closed-form expressions of first and second moments 
and IRFs. Although Den Haan and De Wind (2012) question the suitability of this approach, it has been widely accepted as the efficient and reliable way to get the solution of higher-order approximated DSGE model.

The model solved using third-order approximation with pruning is given by the following state space representation:

$$
\begin{aligned}
& \mathbf{Y}_{\mathbf{t}}=\mathbf{G}\left(\mathbf{X}_{\mathbf{t}}, \sigma\right)+\boldsymbol{\epsilon}_{\boldsymbol{t}}, \\
& \mathbf{X}_{\mathbf{t + 1}}=\mathbf{H}\left(\mathbf{X}_{\mathbf{t}}, \sigma\right)+\sigma \boldsymbol{\eta}_{\boldsymbol{t}+\mathbf{1}},
\end{aligned}
$$

where $\mathbf{X}_{\mathbf{t}}$ denotes a set of state variables that contain pre-determined endogenous and exogenous variables, $\mathbf{Y}_{\mathbf{t}}$ is a set of observable variables, $\boldsymbol{\epsilon}_{\boldsymbol{t}+\mathbf{1}} \sim$ i.i.dN $\left(0, \mathbf{R}_{\epsilon}\right)$, and $\boldsymbol{\eta}_{\boldsymbol{t + 1}} \sim$ i.i.dN $\left(0, \mathbf{R}_{\eta}\right)$. The detailed expression is given in Appendix B.3.

We calibrate some parameters based on Iacoviello and Neri (2010). Table 2.3

describes our calibration. The calibrated parameters $\varepsilon_{p}, \varepsilon_{c, w}^{L}, \varepsilon_{h, w}^{L}, \varepsilon_{c, w}^{B}$, and $\varepsilon_{h, w}^{B}$ in the CES aggregator allow price and wage markups to be 1.2 in the steady state. Additionally, we calibrate $\kappa$. The parameter $\kappa$ is set to match the U.S. homeownership rate of households whose income is below the median as in Gazzani (2016). For ease of implementation, we solve the model using Dynare (Adjemian et al., 2011).

\subsubsection{Estimation}

We use a two-step procedure to estimate the structural parameters which are not calibrated. First, we use the random-walk Metropolis-Hastings algorithm to estimate the model without stochastic volatility process. Since the approximately exact likelihood evaluation methods such as the particle filter and the Gaussian mixture filter are infeasible for a practical purpose due to a huge dimension of state variables, we use the CDKF for the evaluation of the likelihood within the MCMC algorithm. Although the CDKF is based on Gaussian-based approximation, it is computationally 
efficient and reasonably accurate (Andreasen, 2013; Noh, 2018). Most of the priors are based on Iacoviello and Neri (2010). The difference is that for Calvo parameters, we use estimation results obtained from Sun and Tsang (2017). We also allow for a normal distribution for parameters that determine the elasticity of substitution between home-owning and renting $\left(\xi_{h}\right)$. The prior mean for that parameter is set to 1.5 with a loose standard deviation. Since it is hard to identify the correlation of the inflation objective uncertainty shock $\rho_{\sigma_{s}}$ and the standard deviation of the inflation objective uncertainty shock $\sigma_{\sigma_{s}}$, we estimate the exogenous volatility process of the inflation target shock, separately. The first step is to extract the structural inflation target shocks from a VAR model, based on the identification strategy proposed in Mumtaz and Theodoridis (2017). The basic idea of the identification scheme is to extract the shocks that make the largest contribution of the forecast error variance of a measure of long-horizon inflation expectations. As a measure of long-horizon inflation expectations, we use long-horizon personal consumption expenditure inflation expectations from 1977Q1 to 2008Q4. This measure is used in the Federal Reserve board model ${ }^{10}$. Using a series of the identified inflation target shocks, we estimate the volatility process of the inflation target shock. The parameters $\rho_{\sigma_{s}}$ and $\sigma_{\sigma_{s}}$ are to 0.956 and 0.258 , respectively.

In the second step, we use Simulated Method of Moments (SMMs) to estimate the parameters in the stochastic volatility processes. The reason for applying this twostep procedure is two-fold. First, the solution with pruning involves a large number of state variables so that the CDKF is burdensome when estimating the model with stochastic volatility processes. Secondly, by focusing on the parameters in volatility process to match the actual data moments, we can gauge the impacts of volatility shocks on economic activity. The model is estimated using 8 observable variables: real consumption, real residential investment, real business investment, real Shiller

\footnotetext{
${ }^{10}$ We obtain PTR from FRB/US model webpage.
} 
house price index, nominal interest rates, inflation, wage inflation in the consumption sector, and wage inflation in the housing sector. The data span from 1977Q1 to 2008Q4. The details of the data are given in Appendix B.6. Here we briefly describe the estimation procedure.

First Step: We employ the CDKF for the likelihood evaluation within MCMC algorithm. Since the CDKF based on Stirling's interpolation approximates the mean and covariance matrix of state variables using deterministically chosen sigma-points, we call it Sigma-Point Kalman filter. As described in NøRgaard et al. (2000), the CDKF approximates the unknown prediction and filtering density of unobserved state variables using a Gaussian distribution. It implies that it only requires estimating first two moments of the state variables. The estimated first and second moments by the CDKF accurately capture the true moments up to the 3rd order when the state vector is approximately Gaussian. For a non-Gaussian state vector, approximations are accurate up to 2nd order (Andreasen, 2013).

The filter for the equations (2.36) starts by combining the state vector and the exogenous disturbance into a single vector $\chi_{t}^{a}$ with dimension $L$ :

$$
\chi_{t}^{a}=\left[\begin{array}{c}
\mathrm{X}_{\mathrm{t}} \\
\eta_{t+1}
\end{array}\right]
$$

where $\chi_{t \mid t}^{a}=\mathrm{E}\left[\chi_{t}^{a} \mid \mathrm{I}_{t}\right], \chi_{t \mid t-1}^{a}=\mathrm{E}\left[\chi_{t}^{a} \mid \mathrm{I}_{t-1}\right], \boldsymbol{P}_{t \mid t}^{a}=\operatorname{Var}\left[\chi_{t}^{a} \mid \mathrm{I}_{t}\right]$, and $\boldsymbol{P}_{t \mid t-1}^{a}=\operatorname{Var}\left[\chi_{t}^{a} \mid \mathrm{I}_{t-1}\right]$. The next step is to specify $2 L+1$ sigma-points in a way that they capture the full mean and covariance of the augmented state. The $i$ th sigma-points $\chi_{i, t}^{a}$ consists of the $\chi_{i, t}^{x}$ rows which contain state variables and the $\chi_{i, t}^{\eta}$ rows which contain exogenous disturbances. We define sigma-points for filtering steps and prediction steps as 
follows:

$$
\begin{array}{ll}
\text { For prediction: } & \hat{\chi}_{0, t}^{a}=\chi_{t \mid t}^{a} \\
& \hat{\chi}_{i, t}^{a+}=\chi_{t \mid t}^{a}+h \mathrm{~S}_{i, t \mid t}^{a} \text { for } i=1 \ldots L \\
& \hat{\chi}_{i, t}^{a-}=\chi_{t \mid t}^{a}-h \mathrm{~S}_{i-L, t \mid t}^{a} \text { for } i=L+1 \ldots 2 L,
\end{array}
$$

For filtering: $\quad \bar{\chi}_{0, t}^{a}=\chi_{t \mid t-1}^{a}$

$$
\begin{aligned}
& \bar{\chi}_{i, t}^{a+}=\chi_{t \mid t-1}^{a}+h \mathrm{~S}_{i, t \mid t-1}^{a} \text { for } i=1 \ldots L \\
& \bar{\chi}_{i, t}^{a-}=\chi_{t \mid t-1}^{a}-h \mathrm{~S}_{i-L, t \mid t-1}^{a} \text { for } i=L+1 \ldots 2 L,
\end{aligned}
$$

where the scale parameter $h=\sqrt{3}$ for a Gaussian distribution, $S_{i, t \mid t}^{a}$ and $S_{i, t \mid t-1}^{a}$ are the $i$ th column of the upper triangular Cholesky factorization of $\boldsymbol{P}_{t \mid t}^{a}$ and $\boldsymbol{P}_{t \mid t-1}^{a}$ respectively. These sigma-points are propagated through the equations (2.36) for the evaluation of the first and second moments over the prediction and filtering step. We approximately evaluate the likelihood values while implementing the CDKF and embed it into the MCMC algorithm (see the details in Andreasen, 2013; Noh, 2018). We run the random walk Metropolis-Hastings algorithm 200,000 and use 50,000 to evaluate the posterior modes. Prior to estimation, we linearly detrend consumption, residential investment, business investment, and house prices to eliminate low-frequency components of these variables.

Second Step: In order to ensure that the structural model displays features of the actual data, we select the parameters of the stochastic volatility processes (investment technology, labor supply, technology in nonhousing sector, technology in housing sector, housing preference, intertemporal preference, and monetary policy) to match the second moments and the first- and second-order auto-correlations of real consumption, real residential investment, real business investment, real housing price, inflation, nominal 3-month Treasury Bill, and wage inflation in consumption and housing sector via the SMMs exercise. We detrend consumption, residential 
investment, business investment, and house prices using the HP-filter. To compute the moments generated by the model, we first simulate 2,640 samples, starting from the stochastic steady state. We discard the first 2,000 periods as a burn-in and use the last 640 periods to compute the moments.

Formally, we set the following objective function,

$$
\hat{\theta}=\underset{\theta \in \Theta}{\operatorname{argmin}}\left[\frac{1}{T} \Sigma_{t=1}^{T} m\left(x_{t}\right)-\frac{1}{\tau T} \Sigma_{t=1}^{\tau T} m\left(x_{t}(\theta)\right)\right]^{\prime} \mathbf{W}\left[\frac{1}{T} \Sigma_{t=1}^{T} m\left(x_{t}\right)-\frac{1}{\tau T} \Sigma_{t=1}^{\tau T} m\left(x_{t}(\theta)\right)\right],
$$

where $\frac{1}{T} \Sigma_{t=1}^{T} m\left(x_{t}\right)$ is a vector of target standard deviations and first- and secondorder autocorrelations computed from the actual data, $\frac{1}{\tau T} \Sigma_{t=1}^{\tau T} m\left(x_{t}(\theta)\right)$ is a vector of moments obtained from the simulated data, $\theta$ is a vector of parameters in the stochastic volatility processes, and $\mathbf{W}$ is the inverse of the long-run variance of the moments that leads to the smallest asymptotic variance (Ruge-Murcia, 2012). $\tau$ is set to 5 , which is reasonable in terms of statistical and computational efficiency based on the Monte Carlo study in Ruge-Murcia (2012). As suggested in Born and Pfeifer (2014a), we impose informative priors on the parameters of the volatility processes of the consumption technology shock, the investment-specific technology shock, and the monetary policy shock to place parameters in more plausible regions of the parameter space. The target persistence levels of $\rho_{\sigma_{\mu}}, \rho_{\sigma_{k}}$, and $\rho_{\sigma_{R}}$ are $0.760,0.842$, and 0.921 with weight 2000 , respectively. The target standard deviations of $\sigma_{\sigma_{\mu}}, \sigma_{\sigma_{k}}$, and $\sigma_{\sigma_{R}}$ are $0.392,0.304$, and 0.363 with weight 2000 , respectively. The parameter values for $\rho_{\sigma_{\mu}}$ and $\sigma_{\sigma_{\mu}}$ are obtained from Leduc and Liu (2016). The other parameter values $\left(\rho_{\sigma_{k}}, \rho_{\sigma_{R}}, \sigma_{\sigma_{k}}\right.$, and $\left.\sigma_{\sigma_{R}}\right)$ are obtained from Born and Pfeifer (2014a). 
Table 2.4 Prior and Posterior Distribution

\begin{tabular}{|c|c|c|c|}
\hline & & Prior & Posterior \\
\hline \multicolumn{2}{|c|}{ Parameter } & Dist.(mean,std) & Mode(5\%,95\%) \\
\hline$\varepsilon^{L}$ & habit in consumption, lenders & $\operatorname{Beta}(0.5,0.075)$ & $0.3943(0.3331,0.6095)$ \\
\hline$\varepsilon^{B}$ & habit in consumption, borrowers & $\operatorname{Beta}(0.5,0.075)$ & $0.3626(0.2956,0.5586)$ \\
\hline$\eta^{L}$ & labor supply elasticity, lenders & $\operatorname{Gamma}(0.5,0.1)$ & $0.51(0.3015,0.6704)$ \\
\hline$\eta^{B}$ & labor supply elasticity, borrowers & $\operatorname{Gamma}(0.5,0.1)$ & $0.4611(0.3113,0.6743)$ \\
\hline$\xi^{L}$ & labor mobility, lenders & Normal $(1,0.1)$ & $0.8102(0.727,1.1115)$ \\
\hline$\xi^{B}$ & labor mobility, borrowers & $\operatorname{Normal}(1,0.1)$ & $1.0845(0.7725,1.1795)$ \\
\hline$\phi_{k, c}$ & investment adj. cost, consumption & $\operatorname{Gamma}(10,2.5)$ & $15.0139(12.6379,19.7434)$ \\
\hline$\phi_{k, h}$ & investment adj. cost, housing & $\operatorname{Gamma}(10,2.5)$ & $11.9831(7.8272,18.3782)$ \\
\hline$\alpha$ & labor share in production & $\operatorname{Beta}(0.65,0.05)$ & $0.6697(0.5735,0.7505)$ \\
\hline$r_{R}$ & inertia Taylor rule & $\operatorname{Beta}(0.75,0.1)$ & $0.3687(0.2622,0.4439)$ \\
\hline$r_{\pi}$ & inflation resp. Taylor rule & $\operatorname{Normal}(1.5,0.1)$ & $1.5225(1.4106,1.737)$ \\
\hline$r_{Y}$ & output response Taylor rule & $\operatorname{Normal}(0,0.1)$ & $0.3775(0.2792,0.488)$ \\
\hline$\theta_{\pi}$ & Calvo parameters, prices & $\operatorname{Beta}(0.78,0.01)$ & $0.789(0.7625,0.8002)$ \\
\hline$\iota_{\pi}$ & price indexation & $\operatorname{Beta}(0.5,0.2)$ & $0.3063(0.2272,0.9294)$ \\
\hline$\theta_{w, c}$ & Calvo parameters, wages in consumption & $\operatorname{Beta}(0.85,0.01)$ & $0.8632(0.8295,0.8654)$ \\
\hline$\iota_{w, c}$ & wage indexation in consumption & $\operatorname{Beta}(0.5,0.2)$ & $0.9182(0.6803,0.9784)$ \\
\hline$\theta_{w, h}$ & Calvo parameters, wages in housing & $\operatorname{Beta}(0.95,0.01)$ & $0.9671(0.9579,0.9763)$ \\
\hline$\iota_{w, h}$ & wage indexation in housing & $\operatorname{Beta}(0.5,0.2)$ & $0.8688(0.6858,0.957)$ \\
\hline$\zeta$ & utilization parameter & $\operatorname{Beta}(0.5,0.2)$ & $0.9307(0.8573,0.9877)$ \\
\hline$\xi_{h}$ & elasticity of sub. between owning home and rent & $\operatorname{Normal}(1.5,0.5)$ & $1.1797(1.0135,1.7305)$ \\
\hline$\rho_{A C}$ & AR(1) consumption tech. shock & $\operatorname{Beta}(0.8,0.1)$ & $0.9753(0.94,0.9908)$ \\
\hline$\rho_{A H}$ & $\mathrm{AR}(1)$ housing tech. shock & $\operatorname{Beta}(0.8,0.1)$ & $0.9861(0.9645,0.996)$ \\
\hline$\rho_{A K}$ & AR(1) investment tech. shock & $\operatorname{Beta}(0.8,0.1)$ & $0.9238(0.8947,0.9519)$ \\
\hline$\rho_{j}$ & AR(1) housing preference. shock & $\operatorname{Beta}(0.8,0.1)$ & $0.9657(0.9477,0.981)$ \\
\hline$\rho_{z}$ & AR(1) intertemporal shock & $\operatorname{Beta}(0.8,0.1)$ & $0.7906(0.5705,0.9247)$ \\
\hline$\rho_{\tau}$ & AR(1) labor supply shock & $\operatorname{Beta}(0.8,0.1)$ & $0.9803(0.9526,0.9933)$ \\
\hline$\sigma_{A C}$ & std. consumption tech. shock & I. $\mathrm{G}(0.001,0.01)$ & $0.0114(0.0094,0.0146)$ \\
\hline$\sigma_{A H}$ & std. housing tech. shock & I.G(0.001,0.01) & $0.013(0.011,0.0144)$ \\
\hline$\sigma_{A K}$ & std. investment tech. shock & I.G(0.001,0.01) & $0.0096(0.0077,0.0124)$ \\
\hline$\sigma_{j}$ & std. housing preference. shock & I.G(0.001,0.01) & $0.0491(0.0346,0.0669)$ \\
\hline$\sigma_{R}$ & std. interest rate shock & I. $\mathrm{G}(0.001,0.01)$ & $0.0028(0.0023,0.0034)$ \\
\hline$\sigma_{z}$ & std. intertemporal shock & I.G(0.001,0.01) & $0.0115(0.0088,0.0209)$ \\
\hline$\sigma_{\tau}$ & std. labor supply shock & I.G(0.001,0.01) & $0.028(0.0209,0.0332)$ \\
\hline$\sigma_{s}$ & std. inflation target shock & I.G(0.001,0.01) & $0.0005(0.0003,0.0008)$ \\
\hline$\sigma_{m, c}$ & std. measurement err, consumption & I.G(0.001,0.01) & $0.0004(0.0003,0.0026)$ \\
\hline$\sigma_{m, p}$ & std. measurement err, inflation & I.G(0.001,0.01) & $0.0026(0.0023,0.0034)$ \\
\hline$\sigma_{m, w h}$ & std. measurement err, wage in housing & I.G(0.001,0.01) & $0.0054(0.0049,0.0065)$ \\
\hline
\end{tabular}




\subsection{Estimation Results}

\subsubsection{Parameter Estimates}

The posterior modes and $90 \%$ credible sets are reported in Table 2.4 with the prior distributions. The estimated labor income share of borrowers is 0.33 in the thirdorder approximation which is larger than the value implied by the linearized model in Iacoviello and Neri (2010). The estimated degree of habit formation in consumption is marginally larger for lenders than the degree for borrowers $\left(\varepsilon^{L}=0.40\right.$ and $\left.\varepsilon^{B}=0.36\right)$. Although lenders can smooth consumption through saving (capital adjustment and lending to firms and borrowers), they have to take the risks of saving into account and might have large capital adjustment costs. These facts imply that lenders are required to have a large habit persistence to smooth consumption. The parameters $\left(\xi^{L}\right.$ and $\xi^{B}$ ) for labor mobility are 0.81 and 1.08 , respectively, implying imperfect substitutes between the consumption-good market and the housing market. The inverse of the Frisch labor supply elasticity is $0.51\left(\eta^{L}\right)$ and $0.46\left(\eta^{B}\right)$ for lenders and borrowers, respectively. The estimated nominal stickiness for prices and wages is in line with Sun and Tsang (2017), except that price and wage indexation coefficients $\left(\iota_{\pi}, \iota_{w, c}\right.$, $\left.\iota_{w, h}\right)$ are different from their estimates. $\theta_{\pi}=0.79$ implies that retailers reoptimize prices every five quarters. We also find that stickiness in the housing sector $\left(\theta_{w, h}=\right.$ $0.97)$ is higher than in the consumption sector $\left(\theta_{w, c}=0.86\right)$. All shock processes are persistent, ranging from 0.80 to 0.99 .

Table 2.5 summarizes the estimated parameter values for the stochastic volatility processes. A degree of persistence of the volatility processes ranges from 0.57 to 0.88 with monetary policy and intertemporal preference having 0.88 and 0.57 , respectively. The standard deviations of volatility shocks to technology in the nonhousing sector, housing preference, investment-specific technology, and monetary policy are relatively larger than the other shocks. The standard deviation of the volatility shock 
Table 2.5 Estimated Parameter Values

\begin{tabular}{l|l|c}
\hline Parameters & Descriptions & Value \\
\hline$\rho_{\sigma_{c}}$ & Persistence: technology in nonhousing sector & 0.706 \\
$\rho_{\sigma_{h}}$ & Persistence: technology in housing sector & 0.865 \\
$\rho_{\sigma_{j}}$ & Persistence: housing preference shock & 0.776 \\
$\rho_{\sigma_{k}}$ & Persistence: investment-specific technology & 0.804 \\
$\rho_{\sigma_{\tau}}$ & Persistence: labor supply shock & 0.764 \\
$\rho_{\sigma_{z}}$ & Persistence: intertemporal preference shock & 0.578 \\
$\rho_{\sigma_{R}}$ & Persistence: monetary policy shock & 0.883 \\
$\sigma_{\sigma_{c}}$ & STD: technology in nonhousing sector & 0.278 \\
$\sigma_{\sigma_{h}}$ & STD: technology in housing sector & 0.112 \\
$\sigma_{\sigma_{j}}$ & STD: housing preference shock & 0.258 \\
$\sigma_{\sigma_{k}}$ & STD: investment-specific technology & 0.273 \\
$\sigma_{\sigma_{\tau}}$ & STD: labor supply shock & 0.001 \\
$\sigma_{\sigma_{z}}$ & STD: intertemporal preference shock & 0.078 \\
$\sigma_{\sigma_{R}}$ & STD: monetary policy shock & 0.333 \\
\hline
\end{tabular}

Table 2.6 Second Moments in the Model and Data

\begin{tabular}{l|cc|cc}
\hline \multirow{2}{*}{ Variables } & \multicolumn{2}{|c|}{ Model } & \multicolumn{2}{c}{ Data } \\
\cline { 2 - 5 } & STD & AR(1) & STD & AR $(1)$ \\
\hline Consumption & 0.020 & 0.878 & 0.012 & 0.901 \\
Business Investment & 0.053 & 0.797 & 0.043 & 0.933 \\
Residential Investment & 0.097 & 0.752 & 0.101 & 0.947 \\
Inflation & 0.008 & 0.955 & 0.006 & 0.832 \\
Monetary Policy Rate & 0.009 & 0.906 & 0.008 & 0.921 \\
Housing Price & 0.024 & 0.771 & 0.039 & 0.985 \\
Wage Inflation in Cosumption Sector & 0.007 & 0.914 & 0.005 & 0.824 \\
Wage Inflation in Housing Sector & 0.008 & 0.957 & 0.007 & 0.469 \\
\hline
\end{tabular}


for monetary policy shows the largest value. A one-standard-deviation volatility shock increases the standard deviation of technology in the nonhousing and housing sector, housing preference, investment-specific technology, labor supply, intertemporal preference, and monetary policy by $32.0 \%, 11.9 \%, 29.2 \%, 31.4 \%, 0.1 \%, 8.1 \%$, and $39.5 \%$, respectively. These values are calculated by $\left(\exp \left(\eta_{j}\right)-1\right) \times 100$. The small standard deviation of the labor supply volatility shock implies that the labor supply shock process is close to the one of constant volatility. The moments presented in Table 2.6 show that the DSGE model does a good job of matching the standard deviations. In particular, the model approximately captures the volatilities of residential investment, inflation, monetary policy rate, and wage inflation in the consumption and housing sector. However, it does a relatively poor job of reproducing fluctuations in business investment and housing prices and of matching the auto-correlations.

\subsubsection{Impulse Response Analysis}

For the impulse response analysis, we calculate stochastic steady states and generate IRFs around the stochastic steady states of model variables to a joint uncertainty shock following Born and Pfeifer (2014a). We define an uncertainty shock as a twostandard deviation increase in the shock's volatility while keeping level shocks constant. Figure 2.7 shows the impulse responses to two-standard deviation shocks for all volatility processes in the model. To test the role of the third order approximation, we plot the responses based on both the second order approximation and the third order approximation. This shows that uncertainty shocks play no significant role in the second order solution, but the impacts are significantly amplified through third order terms of the solution.

Consistent with the existing literature, uncertainty shocks tend to dampen economic activity. Consumption and business investment fall, reaching a trough of 0.05\% at around the 5-quarter horizon. Housing prices follow a similar pattern, falling by 
Figure 2.7: Responses to a Joint Uncertainty Shock
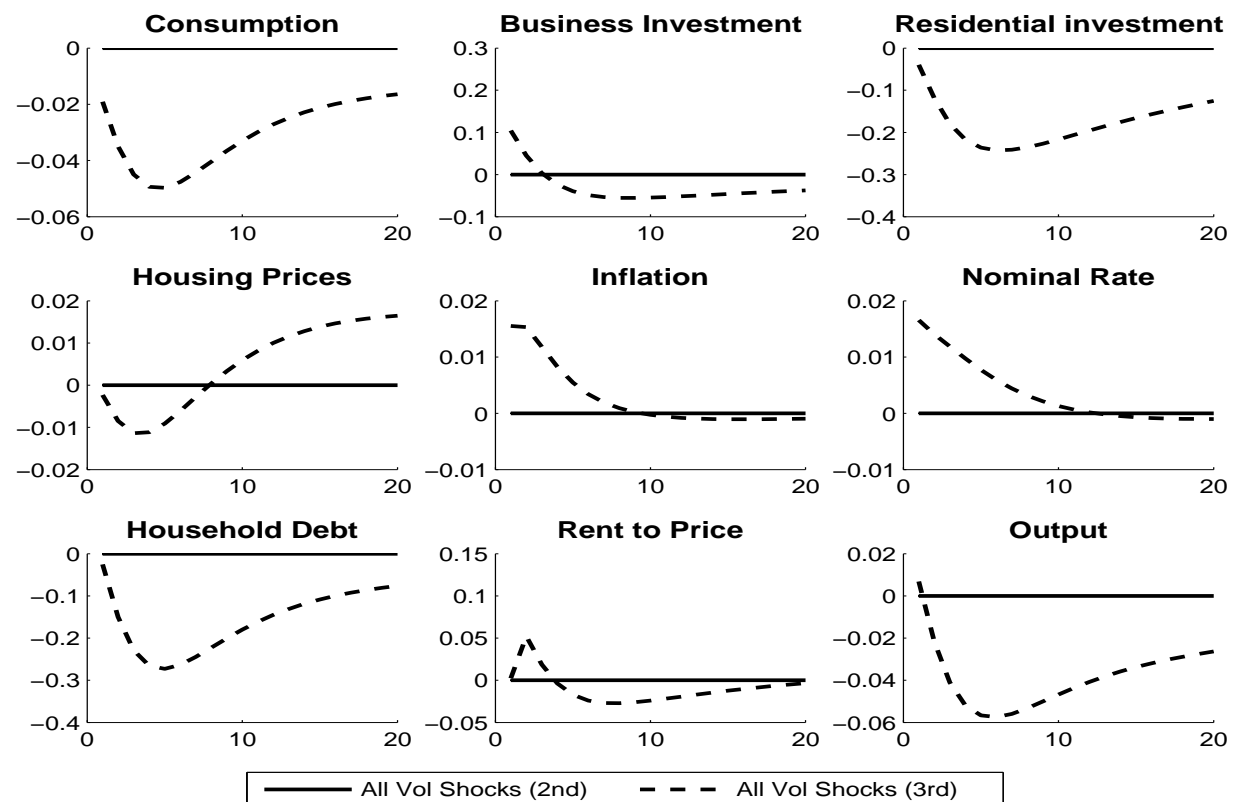

about $0.01 \%$. There is a sharp fall in residential investment in response to the shock, reaching a trough of $0.25 \%$ at around the 5-quarter horizon. A joint uncertainty shock has countervailing initial impacts on business investment and residential investment. The shock initially increases the option value of business investment but reduces the option value of residential investment. The increase in exogenous uncertainty shocks leads households to reduce consumption due to precautionary motives and to decrease the demand for housing due to the financial risk and debt risk effect. Although the model tends to reproduce most of the average impulse responses obtained from the constant VAR model (see Appendix B.8), it mainly fails to capture the empirical responses of inflation and the nominal interest rate. ${ }^{11}$ Under a sticky price and wage, an

\footnotetext{
${ }^{11}$ The house price uncertainty shocks in the constant VAR model initially reduces consumption and residential investment by $0.2 \%$ and $0.9 \%$, respectively. Both of the responses are quite persistent, with their peak responses occurring after about 20 quarters. The peak decline in residential investment is around four times as large as the peak decline in consumption. Inflation falls, though it is not statistically significant. The monetary policy rate drops and remains below the trend for about three years. The decrease in the monetary policy rate leads to a fall in mortgage rates but to a smaller extent, reducing the mortgage spread. Real house prices and mortgage debt decrease by $1.0 \%$ and $2.5 \%$ at the peak, respectively.
} 
upward pricing bias channel leads firms and labor unions to optimally choose higher prices and higher wages, respectively (Fernández-Villaverde et al., 2015). This channel induces an increase in inflation. In addition, the Taylor rule in the model fails to replicate the VAR results. The model-implied reaction of the monetary authority does not offset the negative effects of the uncertainty shocks because of the rise in inflation. A decrease in output can be explained by a rise in markups between wholesale good prices and final good prices $\left(\frac{P_{t}}{P_{t}^{W}}\right)$ with the presence of nominal rigidities. ${ }^{12}$

\subsubsection{Source of House Price Uncertainty}

\section{Variance Decomposition}

In this section, we measure the contribution of each of the eight volatility shocks in our model to fluctuations of the main aggregate variables. In particular, we focus on house price fluctuations. By measuring the contribution of each uncertainty shock to house price fluctuations, we can identify a potential major source of house price uncertainty shocks. Since it is hard to correctly divide total variance among the eight shocks as implemented in the linearized model because of the second and third order terms in the third-order approximation, we calculate the standard deviations of the main aggregate variables generated from the following specifications.

We study five macro-aggregates: consumption $(C)$, business investment $(I K)$, residential investment $(I H)$, housing prices $(q)$, and rent to house price ratio $\left(q^{r} / q\right)$. Using these, we explore four scenarios, turning on: (1) eight uncertainty shocks; (2) supply-side uncertainty shocks; (3) demand-side uncertainty shocks; and (4) an individual uncertainty shock.

Table 2.7 shows that a simultaneous one-standard deviation shock for all sources

\footnotetext{
${ }^{12}$ The upward pricing channels with sticky price and wage tend to initially increase $P_{t}$. In addition, a fall in wages driven by the precautionary labor supply tends to reduce $P_{t}^{W}$ so that markups are increased in the long run.
} 
Table 2.7 Variance Decomposition

\begin{tabular}{l|l|c|c|c|c|c}
\hline & Uncertainty shocks & $C$ & $I K$ & $I H$ & $q$ & $q^{r} / q$ \\
\hline \multirow{5}{*}{ Supply } & Investment tech. & 0.0261 & 0.1762 & 0.1065 & 0.0356 & 0.0226 \\
& Tech. in housing & 0.0004 & 0.0008 & 0.0350 & 0.0068 & 0.0047 \\
& Tech. in nonhousing & 0.0103 & 0.0208 & 0.0658 & 0.0093 & 0.0077 \\
& Labor supply & 0.0001 & 0.0001 & 0.0002 & 0.0000 & 0.0000 \\
\cline { 2 - 7 } & Supply-side uncertainty shocks & 0.0279 & 0.1768 & 0.1292 & 0.0373 & 0.0245 \\
\hline \multirow{5}{*}{ Demand } & Housing pref. & 0.0144 & 0.0094 & 0.1797 & 0.0540 & 0.0556 \\
& Intertemporal pref. & 0.0008 & 0.0014 & 0.0018 & 0.0006 & 0.0005 \\
& Inflation target & 0.0709 & 0.1805 & 0.2044 & 0.0264 & 0.0407 \\
& Monetary policy & 0.0056 & 0.0122 & 0.0256 & 0.0043 & 0.0071 \\
\cline { 2 - 7 } & Demand-side uncertainty shocks & 0.0713 & 0.1800 & 0.2704 & 0.0605 & 0.0690 \\
\hline & All uncertainty shocks & 0.0774 & 0.2538 & 0.3028 & 0.0717 & 0.0737 \\
\hline
\end{tabular}

of uncertainty has significant impacts on business investment and residential investment and has relatively small impacts on consumption, housing prices, and the rent to price ratio. The impacts on both business investment and residential investment are more driven by the demand-side uncertainty shocks than the supply-side uncertainty shocks. Especially, the inflation target uncertainty shocks have the largest impacts on business and residential investment. Also, house price fluctuations are more likely to be explained by the demand-side uncertainty shocks than the supply-side uncertainty shocks. Among all the uncertainty shocks, uncertainty shocks to investment-specific technology, housing preference, and inflation target are the key drivers of the variance in housing prices. Uncertainty shocks to both the technology in the housing sector and the monetary policy play a moderate role in explaining the variance in housing prices.

\section{Supply-side Uncertainty Shocks}

We first examine how economic agents respond to supply-side uncertainty shocks. Figure 2.8 shows impulse responses to (1) uncertainty shocks to investment-specific technology (black solid line), (2) uncertainty shocks to investment-specific technology and technology in the housing sector (black dashed line), (3) uncertainty shocks to investment-specific technology and technology in the nonhousing and housing sector 
(black dashed-dotted line), and (4) uncertainty shocks to investment-specific technology, technology in the nonhousing and housing sector, and labor supply (black dotted line).

The supply-side uncertainty shocks have negative impacts on consumption, residential investment, housing prices, and household debt with the investment-specific uncertainty shock generating the largest impacts. By precautionary motives, consumption initially falls below its steady state and then reverts to the steady state. Residential investment is also affected by the supply-side uncertainty shocks. It initially declines by $0.15 \%$ because of a drop in demand for housing and hence a decrease in the real-options value of investing in new housing construction. The decrease in demand for housing might be induced by the financial risk and debt risk effect, which implies that households who face greater house price uncertainty require a lower housing price. This leads house prices to initially fall below the steady state by $0.04 \%$, and the rent to price ratio to rise by $0.04 \%$. Although the uncertainty shocks to technology in the housing sector increase house prices, its impact is not significant. The level of household debt is diminished through the channel of collateral constraint. Most of these results are closely linked to the key features of the empirical impulse responses in recessionary regimes obtained from the MS-VAR model.

However, the supply-side uncertainty shocks initially increase business investment by $0.2 \%$, which is mostly driven by the investment-specific uncertainty shock. This supports the idea that the investment-specific uncertainty shock increases the real-options value of a new business investment project. Although the uncertainty shocks to technology in the nonhousing sector and labor supply reduce business investment, the magnitude is much smaller than those generated by the investment-specific uncertainty shock. The investment-specific uncertainty leads output to rise after the shock. This implies that the positive real-options effect on business investment dominates the negative effects of markup channels. The supply-side uncertainty shocks 
increase inflation because the upward pricing channels lead to a higher price and a higher wage. The nominal interest rate is elevated by the rise in inflation and output.

\section{Demand-side Uncertainty Shocks}

In this section, we examine how economic agents respond to demand-side uncertainty shocks. Figure 2.9 shows impulse responses to (1) housing preference uncertainty shocks (black solid line), (2) housing preference and intertemporal preference uncertainty shocks (black dashed line), (3) housing preference, intertemporal preference, and inflation target uncertainty shocks (black dashed-dotted line), and (4) housing preference, intertemporal preference, inflation target, and monetary policy uncertainty shocks (black dotted line). The impulse responses are mainly driven by both the housing preference uncertainty shocks and the inflation target uncertainty shocks. They have countervailing impacts on residential investment, housing prices, household debt, and rent to price ratio. Interestingly, the responses of these variables to the house price uncertainty shocks, when compared with the responses to the supplyside uncertainty shocks, are the opposite. However, the inflation target uncertainty shocks generate similar responses to those obtained from the supply-side uncertainty shocks.

The housing preference uncertainty shocks lead households to demand more housing consumption through a hedging effect. When households face higher house price uncertainty, induced by the housing preference uncertainty shocks, the costs for future housing consumption increase. Households can hedge against future housing consumption risk by holding onto their current house, implying that they will pay higher housing prices. The rise in housing prices induced by the housing preference uncertainty shocks leads households to borrow more debt and consume more, due to the collateral constraint and wealth effect. Since housing prices increase larger than rental rates, the rent-to-price ratio falls below its steady state. The collateral con- 

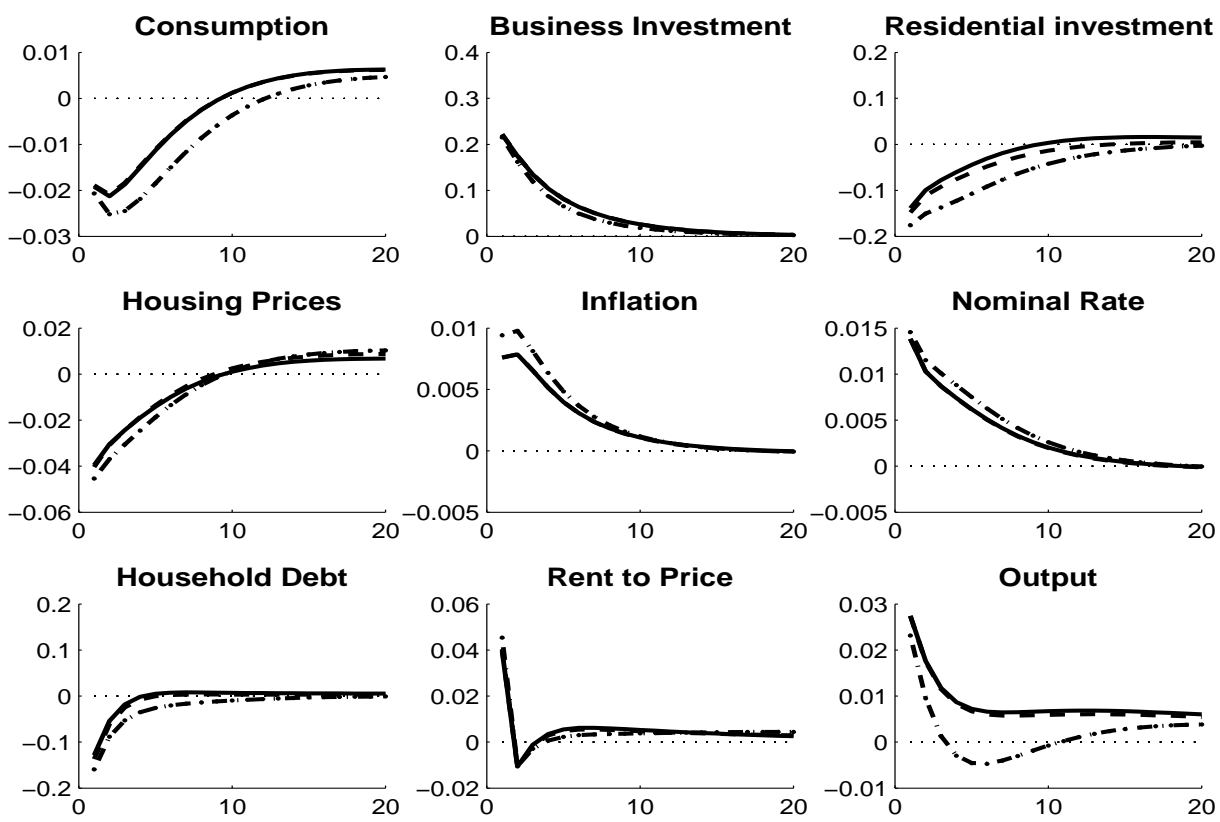

_ Investment tech. - - - +Tech. in housing : - - '+Tech. in nonhousing • + + Labor supply

Figure 2.8: Responses to Supply-side Uncertainty Shock
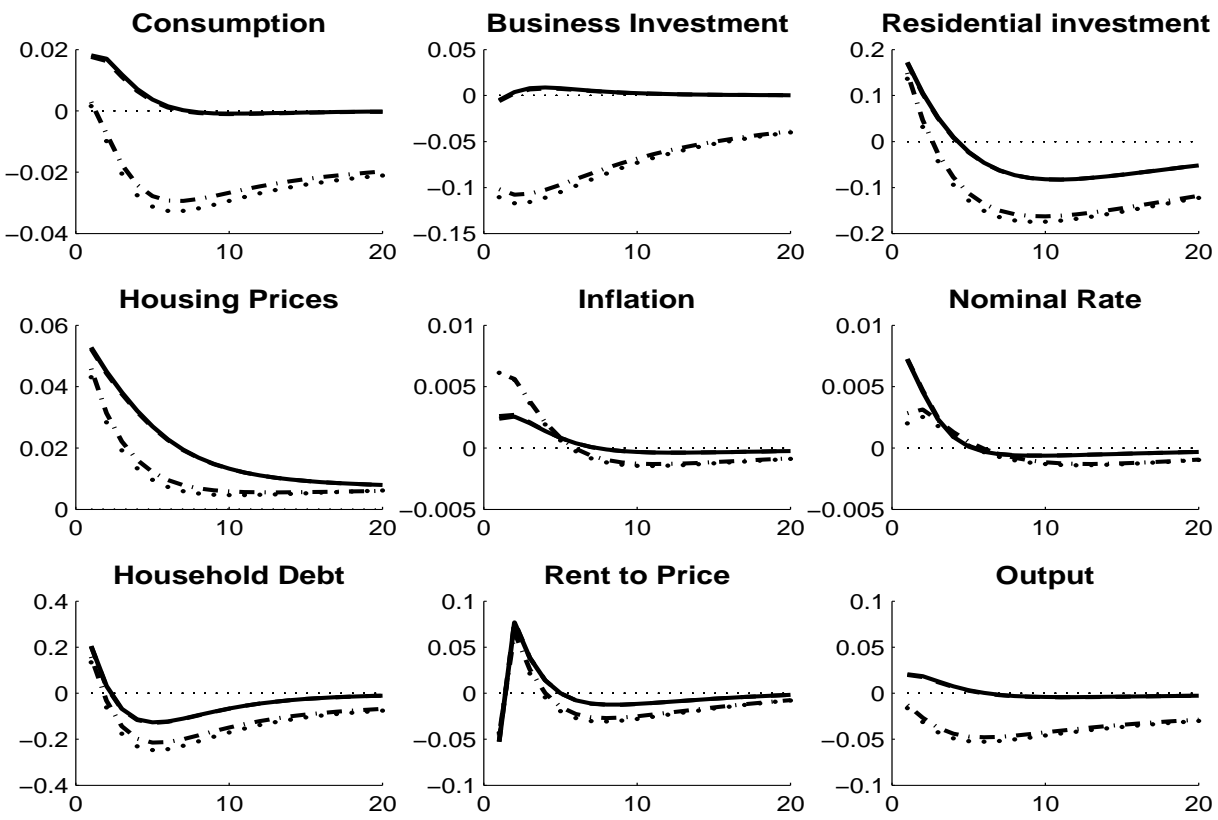

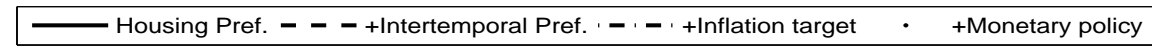

Figure 2.9: Responses to Demand-side Uncertainty Shock 
straint channels and the wealth effect channels tend to dominate the households' precautionary behavior so that they initially increase consumption, though it marginally responds to the house price uncertainty shocks and reverts back to the steady state in the long-run. Inflation and the nominal interest rate increase due to the upward pricing bias channels with sticky prices and wages. As in the case of the supply-side uncertainty shocks, the housing preference uncertainty shocks initially increase output, but for different reasons. The initial rise in output to the housing preference uncertainty shocks is driven by the initial jumps in consumption and residential investment. This implies that they initially dominate the negative effects of markup channels.

Contrary to the housing preference uncertainty shocks, shocks to inflation target uncertainty and monetary policy uncertainty have negative impacts on economic activities. The shocks reduce consumption, business investment, residential investment, housing prices, household debt, and output. Especially, the shocks to inflation target uncertainty show significant impacts on these variables. The results obtained from the housing preference uncertainty shocks are closely related to the VAR impulse responses in expansionary regimes, but the shocks to inflation target uncertainty reproduce the VAR impulse responses in recessionary regimes. In housing boom periods, when there was prevalent housing preference uncertainty, the hedging effect might increase housing prices. However, in recessionary periods, high inflation target uncertainty and monetary policy uncertainty might decrease housing prices.

\subsubsection{Analytical Discussions}

\section{Housing Demand Channels}

Why do house prices increase or decrease after uncertainty shocks? Housing demand is impacted by financial risk, housing consumption hedging, and debt risk effects, all 
driven by uncertainty shocks. These effects have countervailing effects on households' demand for housing. In this section, we analyze the Euler equation to understand how the supply-side uncertainty shocks and the demand-side uncertainty shocks affect housing demand differently through a channel of the financial risk effect, the hedging effect, and the debt risk effect. Since borrowers' equilibrium conditions are more general, in the sense that they face borrowing constraints, we focus on the Euler equations for borrowers and also discuss lenders' behavior. The equilibrium conditions, (2.15) and (2.16), for borrowers can be simplified as:

$$
1=E_{t}\left[\Lambda_{t+1} R_{t+1}\right]
$$

where $R_{t+1}=\frac{(1-\delta) q_{t+1}+\frac{\kappa}{1-\kappa}\left(\frac{h_{t}^{B}}{h_{t}^{r}}\right)^{\xi_{h}-2} q_{t+1}^{r}+m q_{t+1} D_{t+1}}{q_{t}}, \Lambda_{t+1}=\beta^{B} \frac{u_{c}^{B}, t+1}{u_{c}^{B}, t}$ indicates the present value of marginal utility of future consumption, $D_{t+1}=\frac{\pi_{t+1} u_{b, t}^{B}}{R_{t} \beta^{B} u_{c^{B}, t+1}^{B}}$ is the present value of borrowing costs relative to the present value of future consumption, $q_{t+1}$ are housing prices, and $q_{t+1}^{r}$ represent rental rates of housing.

The structural uncertainty shocks propagate through this equilibrium condition, implying that they could be the possible sources of uncertainty surrounding housing price, housing rental rate, inflation rate, and the intertemporal marginal rate of substitution. These shocks create the relationship between returns to owning housing stock and uncertainty. Since the model is too complicated to provide insight about how the financial risk effect, the hedging effect, and the debt risk effect work, we make assumptions about the stochastic process and the distribution of the intertemporal marginal rate of substitution, the growth rate of house price, growth rate of rent, and the relative value of debt over the value of consumption based on Han (2013).

For convenience, the intertemporal marginal rate of substitution is assumed to follow

$$
\Lambda_{t+1}=\exp \left(-r_{t}^{f}-\frac{1}{2} \sigma_{\Lambda, t}^{2}+\epsilon_{\Lambda, t+1}\right)
$$


where $\epsilon_{\Lambda, t+1} \mid I_{t} \sim N\left(0, \sigma_{\Lambda, t}^{2}\right)$. The growth rate of housing prices $\left(x_{t+1}^{q}=\ln \frac{q_{t+1}}{q_{t}}\right)$, the growth rate of rent $\left(x_{t+1}^{q^{r}}=\ln \frac{q_{t+1}^{r}}{q_{t}^{r}}\right)$, and the relative value of debt over the value of consumption $\left(x_{t+1}^{D}=\ln D_{t+1}\right)$ are assumed to follow the $\operatorname{AR}(1)$ process.

$$
x_{t+1}^{j}=\alpha_{0}^{j}+\alpha_{1}^{j} x_{t}^{j}+\epsilon_{t+1}^{j},
$$

where $\epsilon_{t+1}^{j} \mid I_{t} \sim N\left(0, \sigma_{j, t}^{2}\right)$ and $j$ indexes housing price $(q)$, rent $\left(q^{r}\right)$, and debt $(D)$. Under these assumptions, we can derive the analytic form of the uncertainty-return relationship for borrowers. The details of the derivation is given in Appendix B.7.

$$
\frac{\partial E_{t}^{B}\left(r_{t+1}\right)}{\partial \operatorname{Var}_{t}^{B}\left(r_{t+1}\right)}=-\frac{\frac{1}{2} B+\rho_{q \Lambda}^{B}+D \rho_{q q^{r}}^{B}+F \rho_{q D}^{B}}{B+2 D \rho_{q q^{r}}^{B}+2 F \rho_{q D}^{B}}
$$

where $r_{t+1}=\ln R_{t+1}, B=\left((1-\delta)+m\left(\frac{\beta^{L}}{\beta^{B}}-1\right)\right), D=\frac{\kappa}{1-\kappa}\left(\frac{h^{B}}{h^{r}}\right)^{\xi_{h}-2}\left(\frac{q^{r}}{q}\right), F=$ $m\left(\frac{\beta^{L}}{\beta^{B}}-1\right), \rho_{q \Lambda}^{B}$ is the correlation between the growth rate of housing prices and the intertemporal marginal rate of substitution, $\rho_{q q^{r}}^{B}$ is the correlation between the growth rate of housing prices and the growth rate of the rental rate, and $\rho_{q D}^{B}$ is the correlation between the growth rate of housing prices and the log of debt over consumption value. Equation (2.42) shows the relationship between the expected return to owning housing stock and the associated uncertainty. The relationship is determined by $\rho_{q \Lambda}^{B}, \rho_{q q^{r}}^{B}$, and $\rho_{q D}^{B}$, implying that house price uncertainty might affect house prices to the extent that it affects nonhousing consumption, housing consumption, and debt level.

In the financial asset pricing literature, the following assumptions are generally accepted: (1) the growth rate of housing prices is negatively correlated with the intertemporal marginal rate of substitution $\left(\rho_{q \Lambda}^{B}<0\right)$, but $(2)$ it is positively correlated with the growth rate of the rental rate $\left(\rho_{q q^{r}}^{B}>0\right)$. With a negative $\rho_{q \Lambda}^{B}$, the growth rate of housing prices falls when the future nonhousing consumption is more valuable. This implies that households are reluctant to buy a house as a financial asset when 
they want more nonhousing consumption. In this case, faced with greater house price uncertainty and, hence, higher uncertainty about future nonhousing consumption, households require more compensation to induce themselves to buy a house. This leads to a decrease in house prices. This mechanism, based on a negative $\rho_{q \Lambda}^{B}$, describes the financial risk effect, which is common to all financial assets. On the other hand, a positive $\rho_{q q^{r}}^{B}$ implies that a housing price increases when households face a high rental rate which can be thought of as future costs for housing. In this case, households want to buy a house to hedge against uncertain future housing costs, so house price uncertainty increases house prices. This mechanism, with a positive $\rho_{q q^{r}}^{B}$, is associated with the hedging effect. The size of the hedging effect depends on the portion of homeowners relative to the portion of renters. Although negative values of $\rho_{q \Lambda}^{B}$ and positive values of $\rho_{q q^{r}}^{B}$ are generally supported by the empirical evidence (Davidoff, 2006 and Sinai and Souleles, 2008), it is not clear that individual uncertainty shock induces similar implications. In addition, we assume $\rho_{q D}^{B}<0$. The negative value of $\rho_{q D}^{B}$ is associated with the debt risk effect, implying that households reduce their demand for housing to reduce their exposure to risk in debt.

In a similar way, the uncertainty-return relationship for lenders is expressed as

$$
\frac{\partial E_{t}^{L}\left(r_{t+1}\right)}{\partial \operatorname{Var}_{t}^{L}\left(r_{t+1}\right)}=-\frac{\frac{1}{2} B+\rho_{q \Lambda}^{L}+C \rho_{q q^{r}}^{L}}{B+2 C \rho_{q q^{r}}^{L}}
$$

where $r_{t+1}=\ln \left(\frac{(1-\delta) q_{t+1}+q_{t+1}^{r}}{q_{t}}\right), B=(1-\delta) \bar{r}$ and $C=\frac{k}{1-k}\left(\frac{h_{t}^{L}}{h_{t}^{r}}\right)^{\xi-2} q \Lambda$. This relationship is derived by the lenders' equilibrium conditions (2.7) and (2.8).

We now investigate the above-described housing demand channels through which house price uncertainty affects house prices. To measure the magnitude and sign of the financial risk effect $\left(\rho_{q \Lambda}\right)$, the hedging effect $\left(\rho_{q q^{r}}\right)$, and the debt risk effect $\left(\rho_{q D}\right)$ for borrowers and lenders, we simulate $q_{t}, q_{t}^{r}, \Lambda_{t}$, and $D_{t}$ and estimate the following 
equations for borrowers

$$
x_{t}^{q}=\beta_{0}^{B}+\beta_{q \Lambda}^{B} \Lambda_{B, t}+\beta_{q q^{r}}^{B} x_{t}^{q^{r}}+\beta_{q D}^{B} x_{B, t}^{D}+v_{B, t}
$$

and for lenders

$$
x_{t}^{q}=\beta_{0}^{L}+\beta_{q \Lambda}^{L} \Lambda_{L, t}+\beta_{q q^{r}}^{L} x_{t}^{q^{r}}+v_{L, t},
$$

where the coefficients $\beta_{q \Lambda}^{i}, \beta_{q q^{r}}^{i}, \beta_{q D}^{i}$ correspond to the financial risk effect $\rho_{q \Lambda}^{i}$, the hedging effect $\rho_{q q^{r}}^{i}$, and the debt risk effect $\rho_{q D}^{i}$, respectively.

These equations can be interpreted as a reduced-form way of capturing the financial risk effect, the hedging effect, and the debt risk effect. Specifications of this kind are similar to those in standard financial asset pricing models that are used to investigate risk-return relationships. For example, the estimated $\beta_{q \Lambda}^{i}$ is often defined as market risks. Empirically, market risk, $\beta_{q \Lambda}^{i}$, is simply the regression coefficient of the asset return on the marginal rate of substitution, which is a function of nonhousing consumption growth. An important advantage of our exercises, based on the simulated data, relative to the actual data-based regressions is that the structural model-based regressions can avoid problems associated with poor quality consumption data. Financial economists often model consumption growth in terms of a set of market factors, such as the return on a broad-based stock portfolio. In this study, we use the simulated marginal rate of substitution between future and current nonhousing consumption instead of using a proxy variable.

We simulate the variables with four possible combinations for borrowers and lenders: (1) the benchmark case with eight uncertainty shocks; (2) the supply-side uncertainty shocks; (3) the demand-side uncertainty shocks; and (4) the individual uncertainty shock. Using 300 simulated data, we estimate the coefficients 500 times and obtain the average coefficients. Table 2.8 shows that most of the cases satisfy the 
Table 2.8 House price uncertainty and Returns to housing demand

\begin{tabular}{|c|c|c|c|c|}
\hline \multicolumn{5}{|c|}{ Borrowers } \\
\hline & Uncertainty shocks & $\beta_{q \Lambda}^{B}$ & $\beta_{q q^{r}}^{B}$ & $\beta_{q D}^{B}$ \\
\hline \multirow{5}{*}{ Supply } & Investment tech. & 0.003 & 0.971 & -0.009 \\
\hline & Tech. in housing & 0.255 & 0.221 & -0.002 \\
\hline & Tech. in nonhousing & -1.076 & 0.922 & -0.052 \\
\hline & Labor supply & 1.100 & 2.199 & 0.033 \\
\hline & Supply-side uncertainty shocks & -0.029 & 0.950 & -0.009 \\
\hline \multirow{5}{*}{ Demand } & Housing pref. & 0.633 & 1.342 & -0.001 \\
\hline & Intertemporal pref. & -0.554 & -0.292 & -0.002 \\
\hline & Inflation target & 0.658 & 2.125 & 0.010 \\
\hline & Monetary policy & 0.017 & 0.399 & 0.0000 \\
\hline & Demand-side uncertainty shocks & 0.091 & 0.561 & -0.002 \\
\hline & All uncertainty shocks & 0.310 & 0.908 & -0.001 \\
\hline \multicolumn{5}{|c|}{ Lenders } \\
\hline & Uncertainty shocks & $\beta_{q \Lambda}^{L}$ & $\beta_{q q^{r}}^{L}$ & \\
\hline \multirow{5}{*}{ Supply } & Investment tech. & -0.890 & 0.070 & \\
\hline & Tech. in housing & -0.312 & 1.075 & \\
\hline & Tech. in nonhousing & 0.347 & 1.022 & \\
\hline & Labor supply & -0.400 & 0.639 & \\
\hline & Supply-side uncertainty shocks & -0.761 & 0.195 & \\
\hline \multirow{5}{*}{ Demand } & Housing pref. & -3.875 & 0.579 & \\
\hline & Intertemporal pref. & -0.812 & 0.126 & \\
\hline & Inflation target & -0.802 & 0.129 & \\
\hline & Monetary policy & -0.099 & 0.375 & \\
\hline & Demand-side uncertainty shocks & -0.579 & 0.411 & \\
\hline & All uncertainty shocks & -0.544 & 0.408 & \\
\hline
\end{tabular}

financial risk effect $\left(\rho_{q \Lambda}^{B}<0\right)$, the hedging effect $\left(\rho_{q q^{r}}^{B}>0\right)$, and the debt risk effect $\left(\rho_{q D}^{B}<0\right)$, except that the uncertainty shocks seem not to generate the financial risk effects for borrowers. ${ }^{13}$ For lenders, the supply-side uncertainty shocks have a larger effect on the financial risk effect than the demand-side uncertainty shocks, but the demand-side uncertainty shocks have a stronger effect on the hedging effect. Specifically, lenders give more weight to uncertainty about investment-specific technology for the financial risk effect and to housing preference uncertainty for the hedging effect. Although borrowers give a significant weight to uncertainty about technology in the nonhousing sector among supply-side uncertainty for the financial risk effect, this effect is diluted by the other supply-side uncertainty shocks so that it plays a small role in explaining the financial risk effect for borrowers. However, uncertainty about housing preference among demand-side uncertainty still plays a significant role in explaining the hedging effect for borrowers. On average, the supply-side uncertainty

\footnotetext{
${ }^{13}$ As a robustness exercise, we experimented with several specifications that included interest rates and wage growth as controls. We found that the results were robust to this exercise.
} 
Table 2.9 Real-options channels

\begin{tabular}{|c|c|c|c|}
\hline \multicolumn{4}{|c|}{ Real Option Channels } \\
\hline & Uncertainty shocks & $\beta_{R_{c} \Lambda}^{L}$ & $\beta_{R_{h} \Lambda}^{L}$ \\
\hline \multirow[t]{2}{*}{ Supply } & $\begin{array}{l}\text { Investment tech. } \\
\text { Tech. in housing } \\
\text { Tech. in nonhousing } \\
\text { Labor supply }\end{array}$ & $\begin{array}{r}0.956 \\
-4.947 \\
-0.750 \\
-0.617\end{array}$ & $\begin{array}{r}-2.391 \\
0.524 \\
-7.783 \\
-8.046\end{array}$ \\
\hline & Supply-side uncertainty shocks & 0.937 & -2.428 \\
\hline \multirow[t]{2}{*}{ Demand } & $\begin{array}{l}\text { Housing pref. } \\
\text { Intertemporal pref. } \\
\text { Inflation target } \\
\text { Monetary policy }\end{array}$ & $\begin{array}{l}-1.292 \\
-0.796 \\
-9.294 \\
-0.898\end{array}$ & $\begin{array}{l}-1.564 \\
-1.245 \\
-6.612 \\
-2.899\end{array}$ \\
\hline & Demand-side uncertainty shocks & -5.974 & -4.472 \\
\hline & All uncertainty shocks & 0.433 & -2.651 \\
\hline
\end{tabular}

shocks have a larger effect on the debt risk effect than the demand-side uncertainty shocks. Most of the supply-side uncertainty shocks, including investment-specific technology uncertainty and uncertainty about technology in the housing and nonhousing sector have debt risk effects.

\section{Real-options channels}

In this section, we make an additional investigation about the relationship between uncertainty and returns to (business or residential) investment. For simplicity, we close adjustment costs for capital and capacity utilization. Based on lenders' optimal decision making on capital in the nonhousing and housing sector, we can derive the following relationship:

$$
\frac{\partial E_{t}^{L}\left(r_{i, t+1}\right)}{\partial \operatorname{Var}_{t}^{L}\left(r_{i, t+1}\right)}=-\frac{\frac{1}{2} R_{i}+\rho_{R_{i} \Lambda}^{L}}{R_{i}} \quad \text { for } i=c, h
$$

where $r_{i, t+1}=\ln \left(R_{i, t+1} z_{i, t+1}+1-\delta_{k}\right)$ for $i=$ nonhousing sector $(c)$, housing sector $(h)$. This implies that investment decisions are determined by a correlation, $\rho_{R_{i} \Lambda}^{L}$, between gross returns to investment $i$ and the intertemporal marginal rate of substitution describing real option channels. If this correlation is positive, it has a positive real option effect on investment. On the other hand, if it is negative, it has a negative real 
option effect on investment. Based on the same procedure for the housing demand channels, we estimate the following equation,

$$
x_{L, t}^{R_{i}}=\beta^{L}+\beta_{R_{i} \Lambda}^{L} \Lambda_{L, t}+v_{L, t} \quad \text { for } i=c, h,
$$

where $x_{L, t}^{R_{i}}=\ln R_{i, t}$, the coefficient $\beta_{R_{c} \Lambda}^{L}$ corresponds to a real option effect on busi-

ness investment $\left(\rho_{R_{c} \Lambda}^{L}\right)$, and the coefficient $\beta_{R_{h} \Lambda}^{L}$ corresponds to a real option effect on residential investment $\left(\rho_{R_{h} \Lambda}^{L}\right)$. As Table 2.9 shows, a joint uncertainty shock has a positive real option effect on business investment. This result is mainly driven by supply-side uncertainty shocks, especially investment-specific technology uncertainty shocks. However, a joint uncertainty shock has a negative real option effect on residential investment. This result is mainly driven by demand-side uncertainty shocks, especially inflation target uncertainty shocks.

\subsubsection{Additional Analysis}

\section{State-level Evidence for financial risk and hedging effect}

To investigate the housing demand channels through which house price uncertainty affects the demand for housing, we mainly focused on investigating the role of the financial risk effect, the hedging effect, and the debt risk effect using simulated data from an estimated DSGE model. This exercise motivates additional empirical analysis based on the U.S. state-level panel data. As emphasized by Del Negro and Otrok (2007), we take into account the possible heterogeneity of house prices across states. We use quarterly data from 2001Q2 to 2017Q2 on house price index, rent price index, and consumption for the 50 U.S states and the District of Columbia. We construct the growth rate of state-level house prices and rent prices by using metro-level house price indices and rent price indices from Zillow. We use residential electricity usage 
to proxy for nondurable consumption, which is suitable for asset pricing implications as suggested by Da and Yun (2010). Residential electricity usage is from the U.S. Energy Information Administration's Electric Power Monthly publication. Since we do not have enough state-level data for debt, we focus on testing the equation (2.45). For the empirical analysis using panel data, we set up the following equation:

$$
x_{i, t}^{q}=\alpha_{i}+\gamma_{t}+\beta_{q \Lambda}^{i} \Lambda_{i, t}+\beta_{q q^{r}}^{L} x_{i, t}^{q^{r}}+v_{i, t},
$$

where $x_{i, t}^{q}$ is the growth rate of state-level house prices, $\Lambda_{i, t}$ is the growth rate of

state-level consumption, $x_{i, t}^{q^{r}}$ is the growth rate of state-level rent prices, and $\alpha_{i}$ and $\gamma_{t}$ represent state and year fixed effects. Table B.1 in Appendix B.8. shows that over all the sample periods, the financial risk effect and the hedging effect hold. Interestingly, before the 2008 financial crisis, the hedging effect, $\beta_{q q^{r}}$, was more severe than the financial risk effect, $\beta_{q \Lambda}$.

\section{The role of price and wage stickiness}

There have been several prominent studies emphasizing the markup channels based on nominal rigidities (Fernández-Villaverde et al., 2011; Gómez-González et al., 2013; Born and Pfeifer, 2014a,b; Cesa-Bianchi and Corugedo, 2018; Fernández-Villaverde et al., 2015; Mumtaz and Theodoridis, 2015; Leduc and Liu, 2016; Bonciani and van Roye, 2016; Basu and Bundick, 2017). Contractionary effects of uncertainty on economic activity are amplified through an increase in markups when the nominal rigidities exist. The major reasons for the increase in markups can be explained by an aggregate demand channel and an upward pricing bias channel (Fernández-Villaverde et al., 2015). The aggregate demand channel implies that the nominal rigidities hinder fully accommodating the lower demand. The upward pricing bias says that firms faced with higher uncertainty optimally choose a high price when nominal rigidities 
are strong. These channels increase markups, and hence decrease output. Figures B.3 and B.4 illustrate the role of nominal rigidities for the supply-side uncertainty shocks and the demand-side uncertainty shocks, respectively. The red-circled line plots the IRFs when we allow flexible prices. The blue-circled line plots the IRFs when we reduce wage stickiness. As the figure shows, wage stickiness is crucial for the responses of residential investment to both supply-side uncertainty shocks and demand-side uncertainty shocks, though price stickiness plays no significant role in amplifying uncertainty shocks. This result is in line with the findings in Iacoviello and Neri (2010). They document that sticky wages are necessary for the fluctuations of residential investment, making them sensitive to the changes in demand conditions. In addition, the figures show that under flexible wages, the response of output is driven by business investment rather than residential investment.

\section{The role of collateral constraints}

As illustrated in Iacoviello and Neri (2010), collateral effects are the key feature that generates a significant response of consumption following a change in housing prices. Since households are limited to borrowing money equal to the value of housing collateral, the change in house prices not only affect the household's balance sheet but also affect borrowing capacity and consumption level. Figures B.5 and B.6 show impulse responses to the supply-side uncertainty shocks and the demand-side uncertainty shocks, respectively, in the presence of strong collateral constraint channels. Both figures show that the effects of uncertainty shocks on consumption are slightly amplified. This finding is in line with several papers that show a positive effect of housing prices on consumption (Case et al., 2005; Campbell and Cocco, 2007; Iacoviello and Neri, 2010). 


\section{The role of perfect factor mobility}

In this section, we examine how uncertainty shocks affect the economy as we relax the assumption of imperfect labor factor mobility, and, instead, allow factors of production to move freely across sectors. In our model, higher uncertainty has a differential impact on sectoral price markups because of asymmetric price rigidity between a consumption sector and a housing sector. Higher uncertainty increases the price markups on the consumption sector with sticky prices through the precautionary labor supply effect, causing consumption-sector wages to fall. However, firms in the housing sector maintain their markups by flexibly adjusting prices when higher uncertainty induces the precautionary labor supply effect and, hence, a drop in marginal costs. Thus, an increase in uncertainty might lead to an increase in real housing prices in the twosector model, with the combination of flexible housing prices and sticky prices in the consumption sector (Katayama and Kim, 2016). However, if factors can flow freely across sectors, nominal wages will equalize across sectors and the response of real housing prices is less amplified. Figure B.8 shows that the responses of residential investment and housing prices to demand-side uncertainty shocks are less pronounced in the economy with perfect labor factor mobility between a consumption sector and a housing sector.

\subsection{Welfare Analysis and Optimal Policy}

\subsubsection{Uncertainty and Welfare}

In this section, we analyze welfare implications for uncertainty. We calculate compensating variations based on two different values of the means of the stochastic volatility processes in the model. The compensating variations are the changes in welfare in terms of the consumption equivalent measure that is the constant fraction 
of consumption that a household should give away in order to avoid the losses of high uncertainty. We calculate the welfare obtained from the estimated uncertainty, $\sigma_{i}$, (benchmark uncertainty) and compare it with the value calculated based on higher uncertainty, $2 \sigma_{i}$, that is two times larger than the estimated mean, where $i$ indexes different shocks. Since the model considered in this paper has a rich stochastic structure, including second moment shocks, we can investigate the role of various sources of house price uncertainty shocks in welfare evaluation. In this study, we focus on the effects of structural uncertainty shocks on welfare.

We calculate welfare conditional on the non-stochastic steady state. Since we are interested in the transitional effects of uncertainty changes, conditional welfare is preferred (Lester et al., 2014). The aggregate welfare function is a weighted sum of lenders' welfare and borrowers' welfare.

$$
V_{t}^{A}=\left(1-\beta^{L}\right) V_{t}^{L}+\left(1-\beta^{B}\right) V_{t}^{B}
$$

where $V_{t}^{L}$ denotes lenders' welfare and $V_{t}^{L}$ denotes borrowers' welfare. At the optimum,

$$
V_{t}^{i}=U\left(c_{t}^{i}, c_{t-1}^{i}, h_{t-1}^{i}, n_{c t}^{i}, n_{h t}^{i}\right)+\beta^{i} E_{t} V_{t+1}^{i} \text { for } i=L, B
$$

The compensating variations are defined as follows:

$$
\begin{aligned}
& C^{L}=\exp \left[\left(1-\beta^{L}\right)\left(V^{L *}-V_{h}^{L}\right)\right], \\
& C^{B}=\exp \left[\left(1-\beta^{B}\right)\left(V^{B *}-V_{h}^{B}\right)\right], \\
& C^{A}=\exp \left[\left(V^{A *}-V_{h}^{A}\right)\right],
\end{aligned}
$$

where $V^{i *}$ is the $i$ th welfare evaluated at the benchmark case, and $V_{h}^{i}$ is the $i$ th welfare evaluated at the high uncertainty case. We consider four different specifications and numerically evaluate the conditional compensating variations: (1) allowing high uncertainty for all shocks; (2) high uncertainty for supply-side shocks; (3) high 
Table 2.10 Compensating Variations: Baseline Model

\begin{tabular}{l|c|c|c}
\hline & \multicolumn{3}{|c}{ Compensating Variations } \\
\cline { 2 - 4 } & Social & Lenders & Borrowers \\
\hline All uncertainty: $\boldsymbol{\sigma}_{\boldsymbol{i}} \uparrow \mathbf{2} \boldsymbol{\sigma}_{\boldsymbol{i}}$ for all $\boldsymbol{i} \in\{\boldsymbol{c}, \boldsymbol{h}, \boldsymbol{j}, \boldsymbol{k}, \boldsymbol{\tau}, \boldsymbol{z}, \boldsymbol{s}, \boldsymbol{R}\}$ & -2.350 & -1.700 & -0.670 \\
\hline Supply-side uncertainty: $\boldsymbol{\sigma}_{\boldsymbol{i}} \uparrow \mathbf{2} \boldsymbol{\sigma}_{\boldsymbol{i}}$ for all $\boldsymbol{i} \in\{\boldsymbol{c}, \boldsymbol{h}, \boldsymbol{k}, \boldsymbol{\tau}\}$ & -1.100 & -0.150 & -0.950 \\
\hline Investment-specific tech. uncertainty: $\sigma_{i} \uparrow 2 \sigma_{i}$ for all $i \in\{k\}$ & -1.180 & -0.058 & -1.130 \\
Tech. uncertainty in housing sector: $\sigma_{i} \uparrow 2 \sigma_{i}$ for all $i \in\{h\}$ & -0.002 & -0.009 & 0.007 \\
Tech. uncertainty in nonhousing sector: $\sigma_{i} \uparrow 2 \sigma_{i}$ for all $i \in\{c\}$ & 0.087 & 0.005 & 0.082 \\
Labor supply uncertainty: $\sigma_{i} \uparrow 2 \sigma_{i}$ for all $i \in\{\tau\}$ & -0.002 & -0.091 & 0.089 \\
\hline Demand-side uncertainty: $\boldsymbol{\sigma}_{\boldsymbol{i}} \uparrow \mathbf{2} \boldsymbol{\sigma}_{\boldsymbol{i}}$ for all $\boldsymbol{i} \in\{\boldsymbol{j}, \boldsymbol{z}, \boldsymbol{s , \boldsymbol { R } \}}$ & -1.260 & -1.550 & 0.290 \\
\hline Housing preference uncertainty: $\sigma_{i} \uparrow 2 \sigma_{i}$ for all $i \in\{j\}$ & -1.860 & -1.530 & -0.330 \\
Intertemporal preference uncertainty: $\sigma_{i} \uparrow 2 \sigma_{i}$ for all $i \in\{z\}$ & 0.140 & -0.0002 & 0.140 \\
Inflation target uncertainty: $\sigma_{i} \uparrow 2 \sigma_{i}$ for all $i \in\{s\}$ & 0.410 & -0.029 & 0.440 \\
Monetary policy uncertainty: $\sigma_{i} \uparrow 2 \sigma_{i}$ for all $i \in\{R\}$ & 0.053 & 0.013 & 0.040 \\
\hline
\end{tabular}

uncertainty for demand-side shocks; (4) high uncertainty for an individual shock.

Table 2.10 shows the conditional compensating variations based on the above specifications. A joint increase in all uncertainty leads to a negative conditional compensating variation in terms of the aggregate social welfare. The negative value is driven by both lenders and borrowers, implying that they prefer higher uncertainty. Lenders benefit from an increase in both supply-side and demand-side uncertainty, but borrowers benefit from an increase in supply-side uncertainty. Although uncertainty is welfare-reducing for risk-averse households, the benefit of higher uncertainty might compensate for unstable streams of consumption, housing consumption, and leisure. The intuitive explanation for this is that the economy can achieve higher welfare by optimally allocating inputs to the "good times" and the "bad times" when there is an increase in uncertainty. For example, firms can invest more to exploit an uncertain economy, and an increase in housing prices, due to households' hedging demand for housing, extends households' borrowing capacity.

In addition, demand-side uncertainty has countervailing effects on lenders' welfare and borrowers' welfare. A rise in demand-side uncertainty increases lenders' welfare but reduces borrowers' welfare. This is mainly driven by housing preference uncertainty and inflation target uncertainty, respectively. Interestingly, an increase in uncertainty about housing preference leads to an increase in welfare for both lenders and borrowers. This implies that a rise in demand for housing and residential in- 
vestment driven by housing preference uncertainty is beneficial for both lenders and borrowers. However, an increase in uncertainty about inflation target and monetary policy is welfare-reducing. Contrary to demand-side uncertainty, a rise in supply-side uncertainty increases both lenders' welfare and borrowers' welfare. This is mainly driven by labor supply uncertainty and uncertainty about investment-specific technology, respectively. Although uncertainty about investment-specific technology reduces housing demand and residential investment, it increases business investment due to the positive real option effect, and hence increases output. A welfare gain driven by the increased business investment and output dominates a welfare loss of risk-averse lenders and borrowers.

\subsubsection{Uncertainty and Optimal Policy}

In this section, we calculate welfare over a range of LTV ratios and mean uncertainty, and find the optimal LTV ratio. We consider housing preference uncertainty, monetary policy uncertainty, and uncertainty about investment-specific technology. The optimal LTV ratio that would maximize welfare is determined by two countervailing forces: on the one hand, a (1) Low LTV ratio causes borrowers to be more credit constrained, so lenders will own most of the housing stock; on the other hand, a (2) High LTV ratio causes indebted households to face a more severe deleveraging process in response to negative shocks. Figure 2.10 shows that, for given estimated parameters, the optimal LTV ratio is 0.68. However, as the measure of average uncertainty about housing preference and monetary policy increases, the optimal LTV ratios decrease. Although aggregate welfare increases as uncertainty about housing preference increases, due to hedging demands for housing, the lower LTV ratios are required because indebted households might go through a deeper deleveraging process in response to negative shocks when housing preference uncertainty is high. On the contrary, the optimal LTV ratio increases as uncertainty about investment-specific 


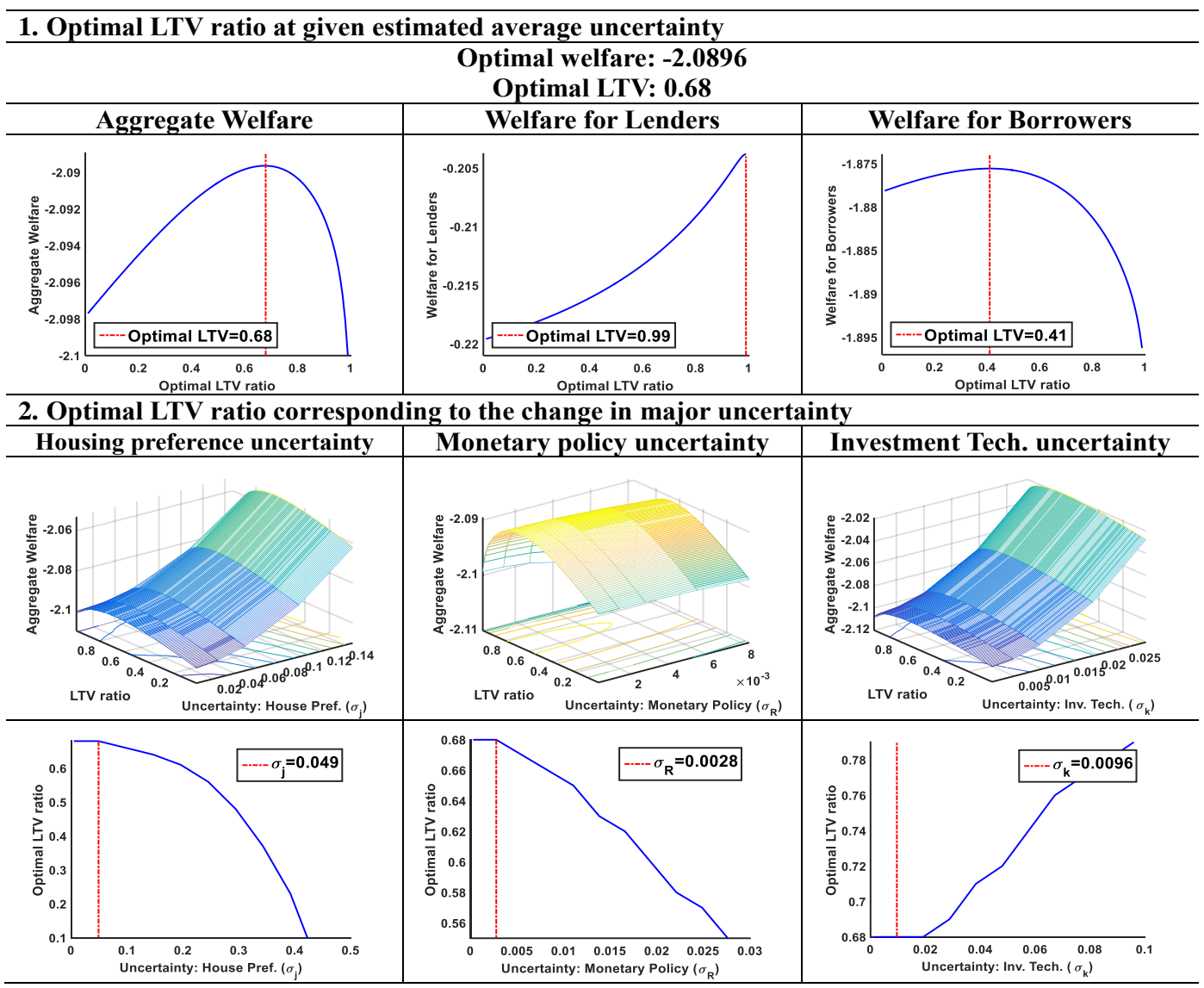

Figure 2.10: Welfare, LTV ratio, and Uncertainty

technology increases due to positive real option effects on business investment.

We also assess the optimal interest rate rules that maximize social welfare depending on mean uncertainty. We find the optimal rules over the parameters ranges $[0,0.8]$ for $r_{R},[1.1,3]$ for $r_{\pi}$, and $[0,3]$ for $r_{Y}$. Table 2.11 shows that the optimal Taylor-type rule does not require interest-rate inertia and features a moderate response to inflation and a weak response to GDP growth. The optimal responses to inflation, interest rate, and GDP growth do not vary as joint uncertainty about all shocks increases. However, the optimal responses to inflation and GDP growth increase as housing preference uncertainty and monetary policy uncertainty increase. In addition, the optimal responses to inflation and GDP growth decrease as uncertainty about investment-specific technology increases. 
Table 2.11 Welfare, Interest Rate Rule, and Uncertainty

\begin{tabular}{l|c|c|c|c}
\hline & Social Welfare & $r_{\pi}$ & $r_{R}$ & $r_{Y}$ \\
\hline All uncertainty: $0.5 \sigma_{i}$ for all $i \in\{c, h, j, k, \tau, z, s, R\}$ & -2.0984 & 2.2 & 0 & 0.2 \\
All uncertainty: $0.7 \sigma_{i}$ for all $i \in\{c, h, j, k, \tau, z, s, R\}$ & -2.0958 & 2.2 & 0 & 0.2 \\
All uncertainty: $\sigma_{i}$ for all $i \in\{c, h, j, k, \tau, z, s, R\}$ & -2.0904 & 2.2 & 0 & 0.2 \\
All uncertainty: $2 \sigma_{i}$ for all $i \in\{c, h, j, k, \tau, z, s, R\}$ & -2.0592 & 2.2 & 0 & 0.2 \\
\hline Housing preference uncertainty: $0.5 \sigma_{i}$ for all $i \in\{j\}$ & -2.0939 & 2 & 0 & 0.1 \\
Housing preference uncertainty: $0.7 \sigma_{i}$ for all $i \in\{j\}$ & -2.0928 & 2.1 & 0 & 0.2 \\
Housing preference uncertainty: $\sigma_{i}$ for all $i \in\{j\}$ & -2.0904 & 2.2 & 0 & 0.2 \\
Housing preference uncertainty: $2 \sigma_{i}$ for all $i \in\{j\}$ & -2.0764 & 3 & 0 & 0.8 \\
\hline Monetary policy uncertainty: $0.5 \sigma_{i}$ for all $i \in\{R\}$ & -2.0904 & 2.2 & 0 & 0.2 \\
Monetary policy uncertainty: $0.7 \sigma_{i}$ for all $i \in\{R\}$ & -2.0904 & 2.2 & 0 & 0.2 \\
Monetary policy uncertainty: $\sigma_{i}$ for all $i \in\{R\}$ & -2.0904 & 2.2 & 0 & 0.2 \\
Monetary policy uncertainty: $2 \sigma_{i}$ for all $i \in\{R\}$ & -2.0913 & 2.5 & 0 & 0.3 \\
\hline Investment-specific tech. uncertainty: $0.5 \sigma_{i}$ for all $i \in\{k\}$ & -2.097 & 3 & 0 & 0.6 \\
Investment-specific tech. uncertainty: $0.7 \sigma_{i}$ for all $i \in\{k\}$ & -2.0949 & 2.9 & 0 & 0.5 \\
Investment-specific tech. uncertainty: $\sigma_{i}$ for all $i \in\{k\}$ & -2.0904 & 2.2 & 0 & 0.2 \\
Investment-specific tech. uncertainty: $2 \sigma_{i}$ for all $i \in\{k\}$ & -2.0635 & 1.8 & 0 & 0 \\
\hline
\end{tabular}

\subsection{Conclusion}

Since the 2008 financial crisis, there has been a surge of uncertainty surrounding the economy. Macroeconomists have been interested in the relationship between uncertainty and economic activity. However, there has been little examination of the effects of house price uncertainty on economic activity, despite considerable house price uncertainty over the past decade along with huge changes in housing prices and dramatic economic fluctuations. This paper investigates the asymmetric effects of U.S. house price uncertainty shocks on economic activity using a reduced-form MSVAR model. The impulse responses to the house price uncertainty shocks in our VAR analysis show that residential investment, housing prices, and mortgage debt decrease by $4 \%, 1 \%$, and $3 \%$, respectively, at the peak in recessionary periods. However, they increase by $1 \%, 0.95 \%$, and $0.85 \%$ at the peak in expansionary periods.

We then use a theoretical DSGE model with a housing sector to find major structural uncertainty shocks that reproduce empirical impulse responses. In particular, we consider an increase in a joint supply-side uncertainty shock and a joint demand-side uncertainty shock. We find that the supply-side uncertainty shocks produce negative impacts on consumption, residential investment, housing prices, and household debt. 
This implies that the supply-side uncertainty shocks induce precautionary motives, a negative real-options effect on residential investment, and a financial risk and debt risk effect on housing demand. These results are mainly driven by the uncertainty shocks to investment-specific technology and replicate the empirical findings in recessionary regimes obtained from the VAR analysis. In contrast, we find that among the demand-side uncertainty shocks, the housing preference uncertainty shocks have positive impacts on consumption, residential investment, housing prices, and household debt that resemble the empirical findings when the economy is in expansionary regimes, but the inflation target uncertainty shocks have the significant opposite impacts on these variables, reproducing the empirical impulse responses in recessionary regimes.

Overall, uncertainty about investment-specific technology and technology in the nonhousing sector, housing preference uncertainty, and inflation target uncertainty play a significant role in explaining fluctuations of housing prices, implying that the shocks to these uncertainty could be the main sources of house price uncertainty shocks. In addition, we investigate the mechanism of how house price uncertainty shocks affect households' demand for housing and housing prices by closely looking at the financial risk effect, the housing consumption hedging effect, and the debt risk effect. Finally, we numerically evaluate welfare changes when there is an increase in uncertainty in the model and find the optimal policy rules over a range of mean uncertainty. The results show that the conditional compensating variations, when there is an increase in all uncertainty, are negative in terms of the aggregate social welfare. This implies that households prefer higher uncertainty. For the given estimated parameters, the optimal LTV ratio is 0.68 . The optimal interest rate rule does not respond to interest-rate inertia, but moderately responds to inflation and weakly responds to GDP growth. 


\section{Chapter 3}

\section{Real Business Cycles in Emerging Countries: The Role of Nonlinearity}

\subsection{Introduction}

Emerging economies have some stylized features of business cycles: (1) they have highly volatile consumption and output, (2) consumption expenditure is more volatile than output, and (3) trade balance is strongly countercyclical. One way to analyze these issues is to apply the class of structural models referred to as dynamic stochastic general equilibrium (DSGE) models. There have been studies on explaining the stylized facts of business cycles in emerging countries based on the DSGE models in different ways (Aguiar and Gopinath, 2007; Garcia-Cicco et al., 2010; Boz et al., 2011; Alvarez-Parra et al., 2013; Chang and Fernández, 2013; Miyamoto and Nguyen, 2017).

Aguiar and Gopinath (2007) argue that business cycles in emerging economies can be well described by shocks to trend productivity using a frictionless real business cycle model. It reflects frequent regime switches in economic policies and market 
failures in emerging economies. On the same line, Boz et al. (2011) impose imperfect information on the model. In contrast, Garcia-Cicco et al. (2010) emphasize financial frictions to capture the business cycle characteristics in emerging economies. In addition, Chang and Fernández (2013) combine shocks to trend productivity with interest rate shocks and financial frictions exemplified by Uribe and Yue (2006). AlvarezParra et al. (2013) divide aggregate consumption expenditure into consumption in nondurable goods and consumption in durable goods. Miyamoto and Nguyen (2017) allow financial frictions and a common shock in the model and estimate the model using the panel data set of 17 countries. However, all of these studies are based on the linearized economy that does not take into account nonlinear factors (e.g., risk factors). In this study, we investigate the role of nonlinearity in explaining the business cycles in emerging countries and find the main structural shocks, focusing on Argentine data. Although there is literature on emphasizing the role of nonlinear terms, there have been little interests in the role of nonlinear factors in explaining business cycles in emerging economies. This study is to fill this gap.

In this study, we use a real business cycle DSGE model for a small open economy. The model is characterized by shocks to trend productivity and financial frictions following Garcia-Cicco et al. (2010). We employ a second-order approximation of the model to take into account risk. As shown in Fernández-Villaverde et al. (2011), shocks to the volatility of real interest rate have a significant impact on the fluctuations of consumption, implying that risk matters for economic fluctuations. Allowing higher order terms will enable the model to match empirical data better. We estimate the nonlinear solution using the GMF with MCMC proposed in Noh (2018). Noh (2018) shows that the GMF with MCMC generates more accurate estimates than the Central Difference Kalman filter and the particle filter, and it is more efficient in terms of computing time than the particle MCMC. We calculate the measure of nonlinearity and identify when the nonlinearity matters. We evaluate the performance 
of the quadratic RBC model by investigating the ability to reproduce the stylized facts. Finally, we find the main structural shocks that explain Argentine economic fluctuations.

Our main findings are the following: (1) the quadratic model with financial frictions does a good job at identifying the parameters of the nonstationary productivity shock process. (2) the data favor the quadratic benchmark RBC and financial-friction models over the linear models. (3) the quadratic model with financial frictions does a better job at capturing serial correlations of the observed data than the linear model with financial frictions. (4) contrary to the linear model with financial frictions, a nonstationary productivity shock in the quadratic model plays an important role in explaining Argentine economic fluctuations.

The remainder of the paper is organized as follows: The first section presents the benchmark RBC model and the augmented RBC model with financial frictions. The second section describes how we estimate the models solved up to second order. The third section discusses estimation results and investigates the role of nonlinearity by comparing the results obtained from the quadratic solution with those from the linearized model. We use estimated parameters, business cycle statistics, variance decomposition, and impulse response analysis for the comparison.

\subsection{Models}

\subsubsection{Benchmark Model}

In this study, we follow the structure of the model proposed by Garcia-Cicco et al. (2010) but estimate the model solved up to second order to investigate the role of nonlinearity in explaining macroeconomic fluctuations in emerging countries. This model is based on the standard small open economy RBC model of Mendoza (1991). 
It has been studied by Schmitt-Grohé and Uribe (2003), Aguiar and Gopinath (2007), Garcia-Cicco et al. (2010) and Chang and Fernández (2013). These studies augmented the standard model of Mendoza (1991) with permanent productivity shocks and foreign interest rate shocks to capture macroeconomic fluctuations in emerging countries. In this model, final-good producers face the following Cobb-Douglas production function with transitory and permanent productivity shocks:

$$
Y_{t}=a_{t} K_{t}^{\alpha}\left(G_{t} h_{t}\right)^{1-\alpha}
$$

where $Y_{t}$ denotes output, $K_{t}$ denotes capital, and $h_{t}$ denotes hours worked. $a_{t}$ and $G_{t}$ are stationary productivity shocks and nonstationary productivity shocks, respectively. The capital letters contain a trend in equilibrium whereas the lower case letters do not have a trend in equilibrium.

The stationary productivity shock $a_{t}$ is assumed to follow a first-order autoregressive process:

$$
\log a_{t+1}=\rho_{a} \log a_{t}+\varepsilon_{t+1}^{a}
$$

where $\varepsilon_{t+1}^{a} \sim \operatorname{iidN}\left(0, \sigma_{a}^{2}\right)$.

The nonstationary productivity shock $G_{t}$ allows for labor-augmenting productivity growth in the model. It is assumed to follow a stochastic trend with the gross growth rate of $g_{t}$. More specifically, let $g_{t}$ be the gross growth rate of $G_{t}$ :

$$
G_{t}=g_{t} G_{t-1}
$$

The natural logarithm of $g_{t}$ follows a first-order autoregressive process:

$$
\log \left(g_{t+1} / \mu_{g}\right)=\rho_{g} \log \left(g_{t} / \mu_{g}\right)+\varepsilon_{t+1}^{g},
$$

where $\varepsilon_{t+1}^{g} \sim \operatorname{iidN}\left(0, \sigma_{g}^{2}\right)$ and $\mu_{g}$ is the deterministic gross growth rate of labor pro- 
ductivity growth. Equation (3.3) implies that the level of productivity follows a unit root process:

$$
\log G_{t+1}=\log G_{t}+\log g_{t+1}
$$

thus, shocks to the growth rate in nonstationary productivity might have a permanent effect on the level of productivity. For example, when the growth rate of the nonstationary component of productivity is highly persistent, a positive shock $\varepsilon_{t+1}^{g}>0$ not only makes the growth rate of labor productivity to be increased through Equation (3.4) but also leads to permanent productivity improvement through Equation (3.5). The permanent increase in productivity can result in permanent income. By the permanent income hypothesis, the shock might have permanent impacts on future consumption so that economic agents smooth consumption by borrowing against future income. In such a case, the shock creates more volatile consumption than output and leads to countercyclical current account (Garcia-Cicco et al., 2010).

In the model, there is an infinitely-lived representative household with the following preference over consumption goods and hours worked. They maximize the expected sum of discounted utilities,

$$
E_{0} \sum_{t=0}^{\infty} \beta^{t} \frac{\left(C_{t}-\theta \omega^{-1} G_{t-1} h_{t}^{\omega}\right)^{1-\gamma}-1}{1-\gamma}
$$

where $0<\beta<1$ denotes the discount factor, $\gamma$ determines the curvature of the utility function, $\omega$ is related to the labor-supply elasticity, $\theta$ determines how much households allocate their time to market work in the steady state, $C_{t}$ is consumption and $E_{0}$ is the expectation operator. The representative household faces the following budget constraint:

$$
\frac{D_{t+1}}{1+r_{t}}=D_{t}-Y_{t}+C_{t}+I_{t}+\frac{\phi}{2}\left(\frac{K_{t+1}}{K_{t}}-\mu_{g}\right)^{2} K_{t}
$$


where $D_{t+1}$ denotes the size of debt in period $t, r_{t}$ denotes the domestic interest rate on bonds held between periods $t$ and $t+1$, and $I_{t}$ denotes gross investment. The last term allows for quadratic capital adjustment costs, and the parameter $\phi$ denotes a degree of capital adjustment costs. The capital stock evolves according to

$$
K_{t+1}=(1-\delta) K_{t}+I_{t}
$$

where $\delta \in[0,1)$ denotes the depreciation rate of capital.

The domestic interest rate is determined by the world interest rate and a debtelastic country's risk premium. The country-specific interest rate premium is an increasing function of detrended aggregate debt. The domestic interest rate is defined as follows:

$$
r_{t}=r^{*}+\psi\left(\exp \left(\frac{\tilde{D}_{t+1} / G_{t}}{\bar{y}}-\bar{d}\right)-1\right)
$$

where $r^{*}$ denotes the world interest rate, $\tilde{D}_{t+1}$ denotes the country's aggregate debt $\left(\tilde{D}_{t+1}=D_{t+1}\right.$ in equilibrium). The domestic interest rate defined above that is the sum of world interest rate and a country's risk premium has been widely utilized in recent studies on RBC models in emerging countries. A debt-elastic interest rate premium ensures that the deterministic steady state is independent of initial conditions as demonstrated by Schmitt-Grohé and Uribe (2003).

Given the processes $\left\{a_{t}\right\},\left\{G_{t}\right\}$ and $\left\{r_{t}\right\}$ the representative household maximizes the utility function (3.6) subject to Equations (3.1), (3.7), (3.8) and the initial conditions $K_{0}$ and $D_{1}$. The household is also subject to a no-Ponzi game constraint of the form

$$
\lim \frac{D_{t+j}}{\prod_{s=0}^{j}\left(1+r_{s}\right)} .
$$

To obtain a stationary competitive equilibrium, we detrend the variables by dividing the level of productivity in the previous period, following Garcia-Cicco et al. 
(2010).

$$
y_{t}=\frac{Y_{t}}{G_{t-1}}, c_{t}=\frac{C_{t}}{G_{t-1}}, i_{t}=\frac{I_{t}}{G_{t-1}}, k_{t}=\frac{K_{t}}{G_{t-1}}, d_{t}=\frac{D_{t}}{G_{t-1}} .
$$

\subsubsection{Financial Frictions}

Based on Uribe and Yue (2006) and Garcia-Cicco et al. (2010), we augment the benchmark RBC model with financial frictions, a country premium shock, a preference shock, and a domestic spending shock. In this model, a representative household maximizes

$$
E_{0} \sum_{t=0}^{\infty} v_{t} \beta^{t} \frac{\left(C_{t}-\omega^{-1} G_{t-1} h_{t}^{\omega}\right)^{1-\gamma}-1}{1-\gamma},
$$

subject to the resource constraint

$$
\frac{D_{t+1}}{1+r_{t}}=D_{t}-W_{t} h_{t}-u_{t} K_{t}+C_{t}+S_{t}+I_{t}+\frac{\phi}{2}\left(\frac{K_{t+1}}{K_{t}}-\mu_{g}\right)^{2} K_{t},
$$

where $W_{t}$ denotes the wage rate, $u_{t}$ denotes the rental rate of capital. $v_{t}$ and $S_{t}$ represent a preference shock and a domestic spending shock, respectively. Firm's objective is to maximize the following profit function:

$$
E_{0} \sum_{t=0}^{\infty} \beta^{t} \frac{\lambda_{t}}{\lambda_{0}}\left[a_{t} K_{t}^{\alpha}\left(G_{t} h_{t}\right)^{1-\alpha}-u_{t} K_{t}-W_{t} h_{t}\left(1+\frac{\eta r_{t}}{1+r_{t}}\right)\right],
$$

where the firm hold non-interest-bearing assets to finance a fraction of the wage bill each period $\left(\kappa_{t} \geq \eta W_{t} h_{t}\right.$ where $\kappa_{t}$ denotes the amount of working capital (see the details in Uribe and Yue (2006))). The assumed working capital incurs the financial cost for the unit labor by $\eta r_{t} /\left(1+r_{t}\right)$ which is a increasing function of $r_{t}$. Following Uribe and Yue (2006), we assume that the initial firm's net liabilities are set to $0 . \lambda_{t}$ denotes the stochastic discount factor (household's marginal utility of wealth). 
We assume that both shocks follow stationary AR(1) process:

$$
\begin{aligned}
& \log v_{t+1}=\rho_{v} \log v_{t}+\varepsilon_{t+1}^{v}, \\
& \log \left(s_{t+1} / \mu_{s}\right)=\rho_{s} \log \left(s_{t} / \mu_{s}\right)+\varepsilon_{t+1}^{s},
\end{aligned}
$$

where $s_{t}=S_{t} / G_{t-1}, \varepsilon_{t+1}^{v} \sim \operatorname{iidN}\left(0, \sigma_{v}^{2}\right)$, and $\varepsilon_{t+1}^{s} \sim \operatorname{iidN}\left(0, \sigma_{s}^{2}\right)$.

The country premium is also augmented with country spread shocks:

$$
r_{t}=r^{*}+\psi\left(\exp \left(\frac{\tilde{D}_{t+1} / G_{t}}{\bar{y}}-\bar{d}\right)-1\right)+\exp \left(\eta_{t}-1\right)-1
$$

where $\eta_{t}$ denotes an exogenous stochastic country premium shock that follows a stationary $\mathrm{AR}(1)$ process:

$$
\log \eta_{t+1}=\rho_{\eta} \log \eta_{t}+\varepsilon_{t+1}^{\eta}
$$

where $\varepsilon_{t+1}^{\eta} \sim \operatorname{iidN}\left(0, \sigma_{\eta}^{2}\right)$.

\subsection{Empirical methodology}

We solve the model up to second order with pruning and calibrate some of the parameters following Garcia-Cicco et al. (2010). Table 3.1 describes our calibration. The model solution is given by the following state-space representation:

$$
\begin{aligned}
& y_{t}=\mathbf{g}\left(x_{t}, \sigma\right)+\epsilon_{t} \\
& x_{t+1}=\mathbf{h}\left(x_{t}, \sigma\right)+\sigma \eta_{t+1}
\end{aligned}
$$

where $x_{t}$ denotes a set of state variables that contains pre-determined endogenous and exogenous variables, $y_{t}$ is a set of observable variables, $\epsilon_{t+1} \sim i . i . d N\left(0, \mathbf{R}_{\epsilon}\right)$, and $\eta_{t+1} \sim$ i.i.dN $\left(0, \mathbf{R}_{\eta}\right)$. We estimate the model using 4 observable variables: output growth, consumption growth, investment growth, and the trade balance-to-output 
Table 3.1 Calibration

\begin{tabular}{c|l|c}
\hline Parameter & Description & Value \\
\hline$\gamma$ & Intertemporal elasticity of substitution & 2 \\
$\omega$ & Labor supply elsasticity & 1.6 \\
$\alpha$ & Capital share of income & 0.32 \\
$\theta$ & Labor parameter & 2.24 \\
$\psi$ & Debt-elastic interest rate parameter & 0.001 \\
$\delta$ & Depreciation rate & 0.1255 \\
$\bar{d}$ & Debt to GDP ratio & 0.037 \\
$r^{*}$ & World interest rate & 0.1 \\
$\mu_{g}$ & Growth rate of nonstationary productivity shock & 1.0107 \\
$\mu_{s}$ & Government spending & $0.1 \bar{y}$ \\
\hline
\end{tabular}

ratio. We use Argentine yearly data of Garcia-Cicco et al. (2010) to ensure that our estimation results can be directly compared with their findings. The dataset spans from 1900 to 2005. We allow for measurement errors in all observable variables whose standard deviations are set to be $25 \%$ of the standard deviations of the observables.

We use the random-Walk Metropolis-Hastings (RWMH) algorithm to estimate the model. Our main interest is to sample parameters from the marginal posterior density $p\left(\theta \mid y_{1: T}\right) \propto p\left(y_{1: T} \mid \theta\right) p(\theta)$ of $p\left(\theta, x_{1: T} \mid y_{1: T}\right)$ using the marginal Metropolis Hastings $(\mathrm{MH})$ algorithm. We can impose prior information through the prior density $p(\theta)$. The ideal marginal likelihood $p\left(y_{1: T} \mid \theta\right)$ is obtained from integrating out state variables $x_{1: T}$.

$$
p\left(y_{1: T} \mid \theta\right)=\prod_{k=1}^{T} p\left(y_{k} \mid y_{1: k-1}, \theta\right)=\prod_{k=1}^{T} \int p\left(y_{k} \mid x_{k}, \theta\right) p\left(x_{k} \mid y_{1: k-1}, \theta\right) d x_{k}
$$

In a linear state-space model with Gaussian distribution assumption, we can calculate the exact form of $p\left(y_{k} \mid y_{1: k-1}, \theta\right)$ by using the Kalman filter.

However, we cannot obtain the analytical form of $p\left(y_{k} \mid y_{1: k-1}, \theta\right)$ in a nonlinear case so that we have to use an approximation method. Among several nonlinear filtering methods, we use the Gaussian mixture filter (GMF) for the evaluation of the 
likelihood $\hat{p}^{G M F}\left(y_{1: T} \mid \theta^{*}\right)$ in the RWMH algorithm with the following acceptance ratio

$$
\hat{r}^{G M F}\left(\theta^{*} \mid \theta\right)=\frac{\hat{p}^{G M F}\left(y_{1: T} \mid \theta^{*}\right) p\left(\theta^{*}\right) q\left(\theta \mid \theta^{*}\right)}{\hat{p}^{G M F}\left(y_{1: T} \mid \theta\right) p(\theta) q\left(\theta^{*} \mid \theta\right)}
$$

Although the GMF is based on Gaussian-based approximation, it is not only computationally efficient but also reasonably accurate (Noh, 2018). The GMF employed in this study is adaptively refined by splitting a mixture component into new mixture components based on Binomial Gaussian mixture. As shown in Noh (2018), the realizations of the PM-MH algorithm converge to the true and stationary density when the likelihood constructed by infinite Gaussian mixtures weakly converges to the true likelihood. This strategy is based on the fact that any continuous density can be obtained by infinitely mixing Gaussian densities in the sense of $L^{1}$ norm with the covariance matrix of every mixture component approaching zero (Alspach and Sorenson, 1971, 1972; Lo, 1972). This fact is based on the Wiener's theorem on approximation (Achieser, 2013).

$$
\int\left|p(x)-\sum_{l=1}^{L} w_{l} N\left(\mu_{l}, \Sigma_{l}\right)\right| d x \rightarrow 0 \quad \text { as } L \rightarrow \infty \text { and } \Sigma_{l} \rightarrow 0
$$

We generate 500,000 draws from the posterior distribution and use 400,000 draws to calculate the point estimates.

\subsection{Results}

This section presents the results from the Bayesian estimation of the standard small open economy RBC model (benchmark RBC model) and the augmented RBC model with financial frictions, using Argentine quarterly data on the growth rates of GDP, private consumption, investment, and trade balance to GDP ratio. 
Table 3.2 Benchmark RBC model

\begin{tabular}{|c|c|c|c|c|c|c|c|c|}
\hline & \multicolumn{8}{|c|}{ Benchmark RBC model } \\
\hline & \multicolumn{2}{|c|}{ Prior Dist. } & \multicolumn{3}{|c|}{ Linear } & \multicolumn{3}{|c|}{ Second-order } \\
\hline & Min & Max & Median & $5 \%$ & $95 \%$ & Median & $5 \%$ & $95 \%$ \\
\hline$\sigma_{g}$ & 0 & 0.2 & 0.039 & 0.027 & 0.049 & 0.039 & 0.031 & 0.046 \\
\hline$\rho_{g}$ & -0.99 & 0.99 & 0.692 & 0.578 & 0.818 & 0.517 & 0.404 & 0.620 \\
\hline$\sigma_{a}$ & 0 & 0.2 & 0.023 & 0.018 & 0.028 & 0.018 & 0.015 & 0.023 \\
\hline$\rho_{a}$ & -0.99 & 0.99 & 0.647 & 0.458 & 0.820 & 0.429 & 0.270 & 0.688 \\
\hline$\phi$ & 0 & 8 & 2.171 & 1.560 & 2.998 & 1.329 & 0.913 & 1.902 \\
\hline \multicolumn{3}{|c|}{ Marginal likelihood } & \multicolumn{3}{|c|}{529.737} & \multicolumn{3}{|c|}{537.1417} \\
\hline
\end{tabular}

\subsubsection{Benchmark RBC model results}

Table 3.2 presents summary statistics on prior and posterior distributions of parameters. Although we use flat priors for all parameters, data seem to be fairly informative about the parameters associated with stochastic shock processes. As pointed out in Garcia-Cicco et al. (2010), since the log-likelihood is relatively flat with respect to the mean productivity growth parameter, $\mu_{g}$, we do not estimate it but calibrate it at 1.005, the value used in Garcia-Cicco et al. (2010). Posterior medians of the persistence parameters of nonstationary productivity shocks and stationary productivity shocks $\left(\rho_{g}\right.$ and $\left.\rho_{a}\right)$ are estimated at 0.69 and 0.65 in a linear model, respectively. However, in a second-order approximation, the persistence levels are lower than those in the linear model. The standard deviations of the shock processes, $\sigma_{g}$ and $\sigma_{a}$, are computed fairly precisely as indicated by the $90 \%$ highest probability density (HPD) intervals. Their posterior medians are 0.04 and 0.023 percent, respectively, in the linear model. The second-order approximation generates a smaller $\sigma_{a}$ than that of the linear model. Also, nonstationary productivity shocks are more volatile than stationary productivity shocks for both linear and quadratic models. The capital adjustment costs parameter $\phi$ in the quadratic model has a posterior median of 1.33 which is smaller than the median value in the linear model. Finally, the marginal likelihood obtained from the quadratic model is larger than that from the linear model, implying that the quadratic model is superior to the linear model. 
Table 3.3 Financial frictions model

\begin{tabular}{c|cc|ccc|ccc}
\hline & \multicolumn{7}{|c|}{ Financial-frictions model } \\
\cline { 2 - 10 } & Prior & Dist. & \multicolumn{3}{|c}{ Linear } & \multicolumn{3}{c}{ Second-order } \\
\hline Parameter & Min & Max & Median & $5 \%$ & $95 \%$ & Median & $5 \%$ & $95 \%$ \\
\hline$\sigma_{g}$ & 0 & 0.2 & 0.009 & 0.001 & 0.034 & 0.031 & 0.009 & 0.040 \\
$\rho_{g}$ & -0.99 & 0.99 & 0.536 & -0.300 & 0.823 & 0.473 & 0.254 & 0.699 \\
$\sigma_{a}$ & 0 & 0.2 & 0.025 & 0.015 & 0.030 & 0.005 & 0.001 & 0.022 \\
$\rho_{a}$ & -0.99 & 0.99 & 0.779 & 0.582 & 0.890 & 0.648 & -0.157 & 0.958 \\
$\sigma_{\nu}$ & 0 & 0.2 & 0.341 & 0.229 & 0.636 & 0.603 & 0.392 & 0.905 \\
$\rho_{\nu}$ & -0.99 & 0.99 & 0.781 & 0.571 & 0.931 & 0.946 & 0.939 & 0.959 \\
$\sigma_{s}$ & 0 & 0.2 & 0.029 & 0.003 & 0.090 & 0.058 & 0.008 & 0.079 \\
$\rho_{s}$ & -0.99 & 0.99 & 0.427 & -0.483 & 0.939 & 0.467 & -0.311 & 0.947 \\
$\sigma_{\mu}$ & 0 & 0.2 & 0.101 & 0.053 & 0.172 & 0.038 & 0.024 & 0.061 \\
$\rho_{\mu}$ & -0.99 & 0.99 & 0.917 & 0.814 & 0.979 & 0.805 & 0.650 & 0.889 \\
$\phi$ & 0 & 8 & 5.358 & 3.306 & 7.559 & 3.992 & 2.403 & 5.768 \\
$\psi$ & 0 & 10 & 1.262 & 0.485 & 2.694 & 0.624 & 0.291 & 1.613 \\
$\eta$ & 0 & 5 & 0.568 & 0.274 & 1.128 & 1.460 & 0.702 & 2.596 \\
\hline Marginal likelihood & \multicolumn{3}{|c|}{563.026} & & \multicolumn{3}{|c}{569.747} & \\
\hline
\end{tabular}

\subsubsection{Financial friction $\mathrm{RBC}$ model results}

In this section, we estimate the augmented RBC model with financial frictions. Specifically we estimate 12 structural parameters, $\rho_{a}, \sigma_{a}, \rho_{g}, \sigma_{a}, \rho_{\nu}, \sigma_{\nu}, \rho_{\eta}, \sigma_{\eta}, \rho_{s}, \sigma_{s}, \phi$, $\psi$ and calibrate the rest. We use the same calibration values as in the benchmark RBC model and use flat priors for all parameters. Table 3.3 presents results from the Bayesian estimation of the augmented model with financial frictions. The first thing to notice is that the posterior distributions of the parameters $\sigma_{g}$ and $\rho_{g}$ of the nonstationary productivity shock process in the linear model are more dispersed than those in the quadratic model. Resorting to higher order approximation induces sensibly more curvature in the likelihood function, and hence increases identifiability of the parameters. The posterior median estimates of the persistence of the stationary productivity shock processes in the linear and quadratic models are 0.78 and 0.65 , respectively, which are significantly larger than the estimated values in the benchmark RBC models. The posterior medians of the persistence of nonstationary productivity shocks are estimated at 0.68 and 0.48 in the linear and quadratic models, respectively. These results indicate that temporary shocks are more persistent 
than permanent shocks in both the linear and quadratic models. The volatilities of temporary and permanent productivity shocks are 0.025 and 0.01 , respectively in the linear model. However, the volatilities of temporary and permanent productivity shocks are 0.005 and 0.031 , respectively in the quadratic model. Although the augmented financial frictions model produces a reduced importance of the nonstationary productivity shock in the linear model, the median of $\sigma_{g}$ in the quadratic model with financial frictions takes the value 0.031 , which is around three times larger than the corresponding point estimate in the linear model, generating the similar implications with Aguiar and Gopinath (2007).

Preference shocks and country spread shocks are relatively persistent with differing degrees of volatilities between the linear and quadratic models. The quadratic model shows more volatile preference shocks than those in the linear model, but the country spread shocks in the quadratic model are less volatile than those in the linear model. The posterior median of the capital adjustment cost parameter, $\phi$, is 5.36 in the linear model which is smaller than the estimate (around 3,99) in the quadratic model. Interestingly, the parameter on country risk premium, $\psi$, has a posterior median of 1.26 in the linear model, but $\psi$ in the quadratic model is 0.62 , implying that the country premium is less sensitive to the level of external debt in the quadratic model than the linear model.

\subsubsection{Performance comparison: predictive marginal likeli- hood}

Conclusions on the superiority between the linear and quadratic versions of the benchmark RBC model and the RBC model with financial frictions imply that the quadratic model prevails marginally (Table 3.2 and 3.3 show that the quadratic model has larger marginal likelihoods than the linear model). In order to have further elements for the comparison between the linear and the quadratic representations, we also compute 

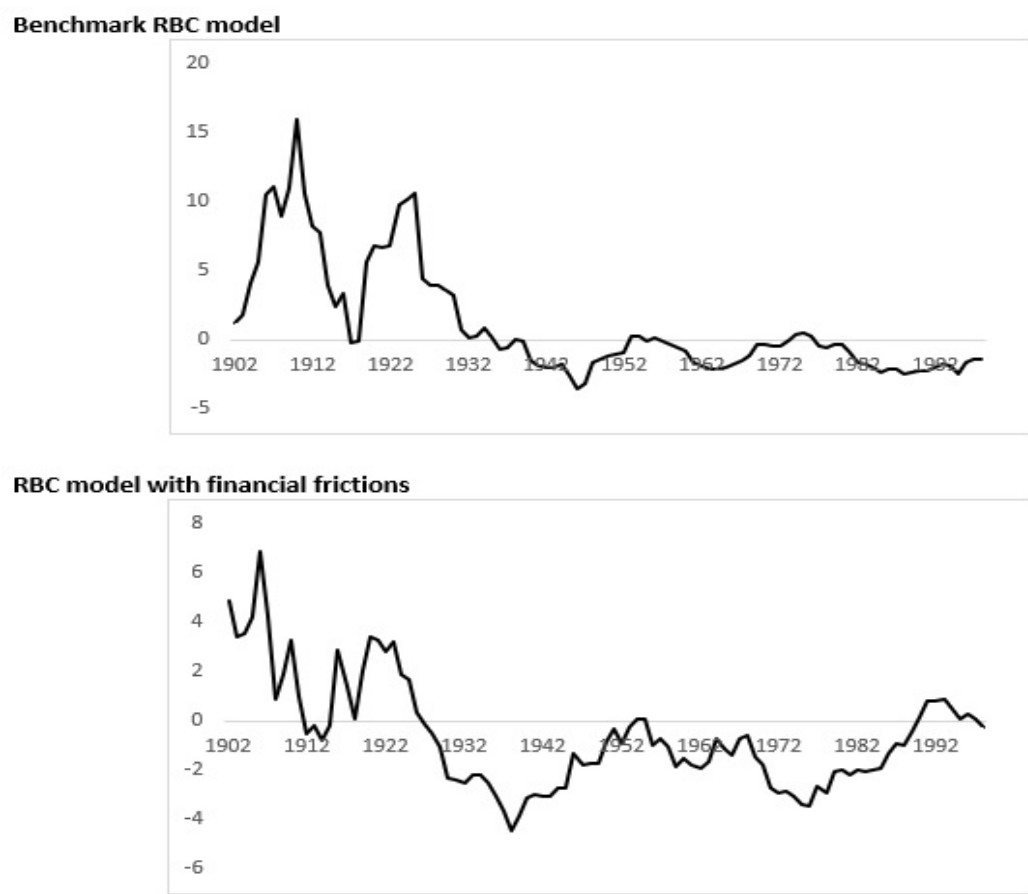

Figure 3.1: Predictive likelihoods

h-step-ahead conditional predictive densities, following Warne et al. (2017):

$$
p\left(y_{t+1: t+h} \mid y_{1: t}, m\right)=\int_{\Theta} p\left(y_{t+1: t+h} \mid y_{1: t}, \theta, m\right) p\left(\theta \mid y_{1: t}, m\right) d \theta
$$

where $m \in\{$ linear,quadratic $\}$. We calculate 8-step-ahead conditional predictive densities in this exercise for both the benchmark RBC model and the financial friction model and plot the following conditional predictive distribution ratio between the linear and quadratic models:

$$
\ln \frac{p\left(y_{t+1: t+8} \mid y_{1: t}, m=\text { quadratic }\right)}{p\left(y_{t+1: t+8} \mid y_{1: t}, m=\text { linear }\right)} \quad \text { for } \quad t=1,2, \ldots, T-8
$$

From this comparison, we see that the conditional predictive distribution ratio is favorable to the quadratic specification over the initial periods from 1902 to late 1920s. However, after 1930, the conditional predictive distribution ratio becomes marginally favorable to the linear specification. Table 3.4 shows that economic fluc- 
Table 3.4 Subperiod standard deviations

\begin{tabular}{c|c|c|c|c}
\hline & $\begin{array}{c}\text { Output } \\
\text { Growth }\end{array}$ & $\begin{array}{c}\text { Conusmption } \\
\text { Growth }\end{array}$ & $\begin{array}{c}\text { Investment } \\
\text { Growth }\end{array}$ & $\begin{array}{c}\text { Trade Balance } \\
\text { to Output ratio }\end{array}$ \\
\hline $1901-1920$ & 6.988 & 8.265 & 27.251 & 5.899 \\
$1921-1940$ & 4.366 & 9.171 & 20.726 & 7.453 \\
$1941-1960$ & 4.957 & 7.691 & 21.402 & 3.690 \\
$1961-1980$ & 3.866 & 5.734 & 11.165 & 2.840 \\
$1981-2005$ & 6.218 & 7.344 & 19.666 & 2.820 \\
\hline
\end{tabular}

tuations are more severe over the periods from 1901 to 1940 than the periods after 1940. This suggests that non-linearities are important in case of very large and persistent shocks, but tend to be less relevant during periods of moderate fluctuations. On the basis of these results, we conclude that both the quadratic versions of the benchmark RBC and financial-friction models are superior to the linear models over the years when economic variables are more distant from their steady-state values, implying that the quadratic model is not always superior to the linear specification. Although the quadratic model tends to reduce parameter identification issues, especially for the parameters of nonstationary productivity shocks, it might fail to predict actual data when economic fluctuations are moderate due to misspecification of the model. Although estimating a non-linear model amounts to extracting more testable implications from the theory, hence achieving more efficient, or less biased, parameter estimates when the model is approximately correct, it might be misspecified for the explanation of the actual data. If the nonlinear model is only a rough approximation of reality, its non-linear implications are likely to make it more at odds with the data.

\subsubsection{Role of nonlinearity}

\section{Measure of nonlinearity}

We use a nonlinearity measure proposed by Raitoharju and Ali-Loytty (2012) after normalization by dividing it by measurement error variance. The measure is based on 


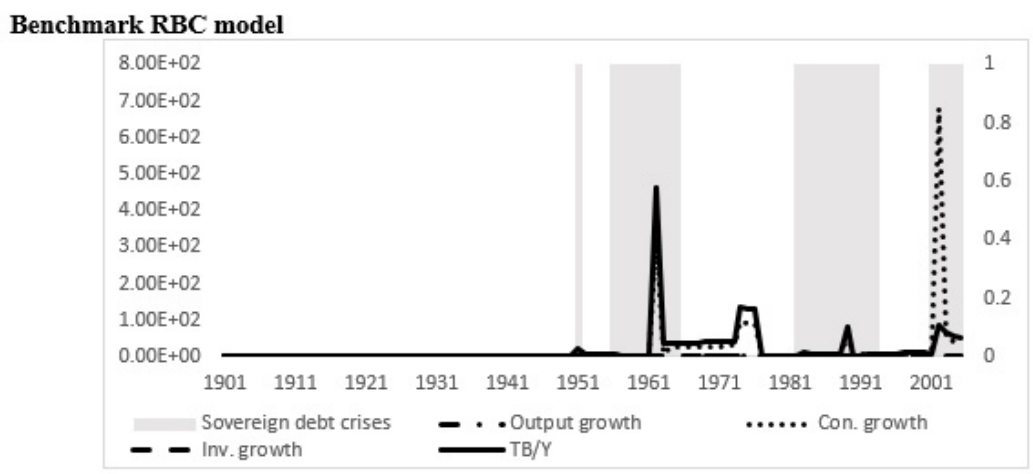

RBC model with financial frictions

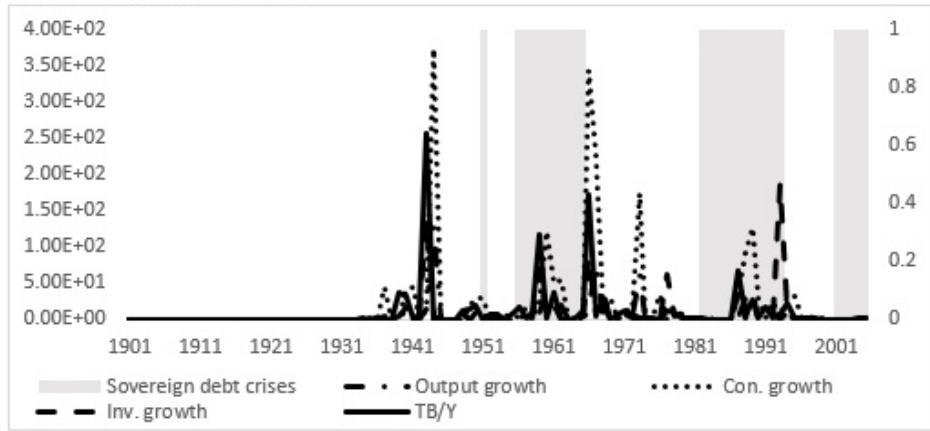

Notes. The shaded area denotes sovereign debt crises obtained from the webpage by Reinhart and Rogoff.

Figure 3.2: Measure of nonlinearity

the comparison between the Extended Kalman filter and the Second Order Gaussian filter:

$$
N L^{l}=\frac{\operatorname{trace}\left(Q^{l} Q^{l}\right)}{h^{4}}
$$

where $l$ indicates the $l$ th measurement equation. $Q^{l}$ is defined as

$$
Q_{[c, d]}^{l}= \begin{cases}g_{l}\left(\chi_{t \mid t-1}^{a}+h \mathrm{~S}_{i, t \mid t-1}^{a}\right)+g_{l}\left(\chi_{t \mid t-1}^{a}-h \mathrm{~S}_{i, t \mid t-1}^{a}\right)-2 g_{l}\left(\chi_{t \mid t-1}^{a}\right) & c=d \\ \frac{1}{2}\left[g_{l}\left(\chi_{t \mid t-1}^{a}+h \mathrm{~S}_{i, t \mid t-1}^{a}+h \mathrm{~S}_{i, t \mid t-1}^{a}\right)+g_{l}\left(\chi_{t \mid t-1}^{a}-h \mathrm{~S}_{i, t \mid t-1}^{a}-h \mathrm{~S}_{i, t \mid t-1}^{a}\right)-\right. & c \neq d \\ \left.2 g_{l}\left(\chi_{t \mid t-1}^{a}\right)-Q_{[c, c]}^{l}-Q_{[d, d]}^{l}\right] & c \neq d\end{cases}
$$

Figure 3.2 shows that the nonlinearity measure tends to increase over sovereign debt crises, implying that second moments matter for risk-averse economic agents' decision making over these periods. Especially, consumption growth and the trade balance to output ratio show more severe nonlinearity than output growth and investment 
Table 3.5 Statistics

\begin{tabular}{|c|c|c|c|c|c|}
\hline & Statistics & $\begin{array}{l}\text { Output } \\
\text { Growth }\end{array}$ & $\begin{array}{c}\text { Consumption } \\
\text { Growth }\end{array}$ & $\begin{array}{c}\text { Investment } \\
\text { Growth }\end{array}$ & $\begin{array}{l}\text { Trade Balance } \\
\text { to Output ratio }\end{array}$ \\
\hline \multicolumn{6}{|c|}{ Standard deviation } \\
\hline \multirow{2}{*}{ Linear } & RBC model & 6.728 & 6.901 & 13.920 & 38.469 \\
\hline & Financial-frictions model & 5.389 & 8.217 & 16.859 & 4.312 \\
\hline \multirow{3}{*}{ Nonlinear } & RBC model & 5.996 & 5.950 & 13.093 & 31.991 \\
\hline & Financial-frictions model & 5.056 & 6.881 & 13.118 & 2.946 \\
\hline & Data & 5.388 & 8.223 & 16.782 & 4.302 \\
\hline \multicolumn{6}{|c|}{ Correlation with output growth } \\
\hline \multirow{2}{*}{ Linear } & RBC model & & 0.933 & 0.808 & -0.086 \\
\hline & Financial-frictions model & & 0.793 & 0.621 & -0.253 \\
\hline \multirow{3}{*}{ Nonlinear } & RBC model & & 0.937 & 0.827 & -0.055 \\
\hline & Financial-frictions model & & 0.857 & 0.677 & -0.199 \\
\hline & Data & & 0.737 & 0.663 & -0.049 \\
\hline \multicolumn{6}{|c|}{ Correlation with $\mathrm{TB} / \mathrm{Y}$} \\
\hline \multirow{2}{*}{ Linear } & RBC model & & -0.091 & -0.088 & \\
\hline & Financial-frictions model & & -0.454 & -0.315 & \\
\hline \multirow{3}{*}{ Nonlinear } & RBC model & & -0.063 & -0.056 & \\
\hline & Financial-frictions model & & -0.324 & -0.126 & \\
\hline & Data & & -0.274 & -0.208 & \\
\hline \multicolumn{6}{|c|}{ Serial correlation } \\
\hline \multirow{2}{*}{ Linear } & RBC model & 0.165 & 0.081 & -0.021 & 0.996 \\
\hline & Financial-frictions model & -0.011 & -0.125 & -0.128 & 0.465 \\
\hline \multirow{3}{*}{ Nonlinear } & RBC model & -0.024 & 0.000 & -0.100 & 0.996 \\
\hline & Financial-frictions model & 0.075 & -0.029 & -0.048 & 0.652 \\
\hline & Data & 0.098 & -0.012 & 0.313 & 0.577 \\
\hline
\end{tabular}

growth. In the following section, we investigate how the nonlinearity generates different implications between the linear and quadratic models.

\section{Basic statistics}

We compare selected key moments implied by the model with their empirical counterparts. Table 3.5 presents the second moments of the observed data and the RBC and financial-friction models (solved up to first- and second-order). We compute standard deviations, first-order autocorrelations and contemporaneous correlations implied by the RBC and financial-friction models and compare them to the observed moments. The RBC model fails to match the observed characteristics in three dimensions. First, output growth and the trade balance to output ratio are more volatile in the RBC model for both the linear and quadratic approximations than the observed data. The standard deviations of the trade balance to output ratio in the models are $38.47 \%$ and $31.99 \%$ in the linear and quadratic models, respectively, whereas the 
standard deviation of the observed data is $4.3 \%$. Second, serial correlations of output growth, consumption growth, and investment growth are different from their empirical counterparts, and trade balance to output has a much higher first autocorrelations estimated at 0.99 for both linear and quadratic models than its observed counterpart 0.58. Third, the RBC model is not successful in reproducing the relatively strong counter-cyclicality of the trade balance to output ratio in U.S. data. The model is relatively successful in mimicking the contemporaneous correlations of output growth with consumption and investment growth. However, the correlation of the trade balance to output ratio with output growth (0.049 in the data vs. 0.086 in the linear $\mathrm{RBC}$ model) and growth rates of aggregate demand components are overestimated by the linear RBC model. However, the quadratic RBC model tends to show the reasonable correlation of the trade balance to output ratio with output growth.

Table 3.5 also shows standard deviations, serial correlations, and contemporaneous correlations of variables implied by the financial frictions model. Similar to the RBC model, the model with financial frictions predicts more volatile consumption growth than output growth in line with the observed data. As in the benchmark RBC model, investment growth is predicted to be less volatile in the financial-friction model (16.78\% in the linear model and $13.22 \%$ in the quadratic model) than the observed data (20.34\%). However, financial-friction model does remarkably well, compared to the RBC model, in predicting the standard deviation of the trade balance to output ratio $(4.30 \%$ in the linear and $3.01 \%$ in the quadratic model compared to $5.14 \%$ in the data). Also in line with the data, the first-order serial correlations of the growth rates of output, consumption, and investment are predicted to be small. In stark contrast to the RBC model, the model with financial frictions perfectly matches the first-order autocorrelation of the trade balance to output ratio. The quadratic model correctly predicts the positive auto-correlation of output growth and the negative auto-correlation of consumption growth. However, the correlation between output 
Table 3.6 Variance Decomposition

\begin{tabular}{l|cccc}
\hline \hline \multicolumn{1}{c|}{ Linear } & $\begin{array}{c}\text { Output } \\
\text { Growth }\end{array}$ & $\begin{array}{c}\text { Conusmption } \\
\text { Growth }\end{array}$ & $\begin{array}{c}\text { Investment } \\
\text { Growth }\end{array}$ & $\begin{array}{c}\text { Trade Balance } \\
\text { to Output ratio }\end{array}$ \\
\hline \hline Nonstationary tech. & $0.02(7.74)$ & $0.02(3.49)$ & $0.03(1.19)$ & $0.0002(0.1)$ \\
Stationary tech. & $0.21(70.1)$ & $0.2(29.89)$ & $0.31(10.96)$ & $0.0013(0.71)$ \\
Preference & $0.02(5.52)$ & $0.17(25.9)$ & $0.85(30.3)$ & $0.01(6.42)$ \\
Country premium & $0.04(14.75)$ & $0.26(39.43)$ & $1.59(56.89)$ & $0.17(92.46)$ \\
Domestic spending & $0.0001(0.02)$ & $0.0007(0.11)$ & $0.004(0.14)$ & $0.0002(0.11)$ \\
\hline All shocks & 0.29 & 0.67 & 2.79 & 0.18 \\
\hline \hline & Output & Conusmption & Investment & Trade Balance \\
\multicolumn{1}{c|}{ Nonlinear } & Growth & Growth & Growth & to Output ratio \\
\hline \hline Nonstationary tech. & $0.13(51.17)$ & $0.13(27.75)$ & $0.23(13.08)$ & $0.0013(1.35)$ \\
Stationary tech. & $0.03(10.99)$ & $0.02(4.48)$ & $0.03(2.01)$ & $0.0009(0.89)$ \\
Preference & $0.04(15.29)$ & $0.14(29.73)$ & $0.74(42.89)$ & $0.02(28.23)$ \\
Country premium & $0.06(21.43)$ & $0.18(36.82)$ & $0.64(37.19)$ & $0.04(49.5)$ \\
Domestic spending & $0.0004(0.08)$ & $0.0019(0.35)$ & $0.01(0.43)$ & $0.0005(0.51)$ \\
\hline All shocks & 0.26 & 0.47 & 1.72 & 0.09 \\
\hline \hline
\end{tabular}

Notes: We calculate the standard deviations (A) of simulated data by each shock. We also calculate the standard deviations (B) of simulated data obtained from turning on all the shocks.

The value in parenthesis is $\mathrm{A} / \mathrm{B}$.

growth and investment growth is underestimated (0.62 and 0.61 in the linear and quadratic models vs. 0.66 in the data). Interestingly, the quadratic model with financial frictions is successful in reproducing a countercyclical trade balance to output ratio. Finally, the correlations of the trade balance to output with consumption growth and investment growth have the correct signs. However, they are significantly larger in the linear model than the data, but they are similar to the data in the quadratic model.

\section{Variance decomposition}

In order to examine the relative role of structural shocks in explaining the variations in observed variables, we compute variance decompositions using the financial-friction model. Results are presented in Table 3.6 for both the linear and quadratic models. For the linear case, stationary productivity shocks explain most of the variations in output growth $(70 \%)$ whereas nonstationary productivity shocks explain about 7.7\%. Approximately $30 \%$ of the variance of consumption growth is explained by stationary productivity shocks, $3.5 \%$ by nonstationary productivity shocks, $26 \%$ by 
preference shocks and $39 \%$ by country premium shocks. Nonstationary productivity shocks have a relatively small share in variations of investment growth (1.2\%) and trade balance to output ratio (about $0.1 \%$ ). Instead, approximately $56.9 \%$ of the variations in investment growth is explained by the variations in country premium shocks. Country premium shocks have the largest share in the variance of tradebalance to output ratio with about $92.5 \%$ followed by preference shocks $(6.4 \%)$ and stationary productivity shocks $(0.71 \%)$.

Overall results similarly apply to the quadratic model with financial frictions. However, nonstationary productivity shocks have a larger role in explaining the fluctuations of output growth, consumption growth, and investment growth than stationary productivity shocks. In addition, preference shocks have a larger role in explaining the fluctuations of investment growth than country premium shocks. These results are surprising because we would expect temporary productivity shocks to have the largest share for output growth and consumption growth. For example, Garcia-Cicco et al. (2010) report that $84 \%$ of the variance of output growth and $51.3 \%$ of the variance of consumption growth are explained by transitory productivity shocks whereas the contributions of permanent productivity shocks to output and consumption growth are small. In addition, they report that the fluctuations of investment growth are mainly explained by country premium shocks. However, country premium shocks still matter for the fluctuations of investment growth and trade balance to output ratio in the quadratic model.

\section{Impulse responses in a benchmark RBC model}

Productivity shocks (one-standard-deviation shocks): Figure 3.3 and Figure 3.4 show impulse responses to stationary and nonstationary productivity shocks, obtained from the linear and quadratic benchmark RBC models. Figure 3.3 shows that a transitory productivity shock tends to produce a positive relationship between out- 


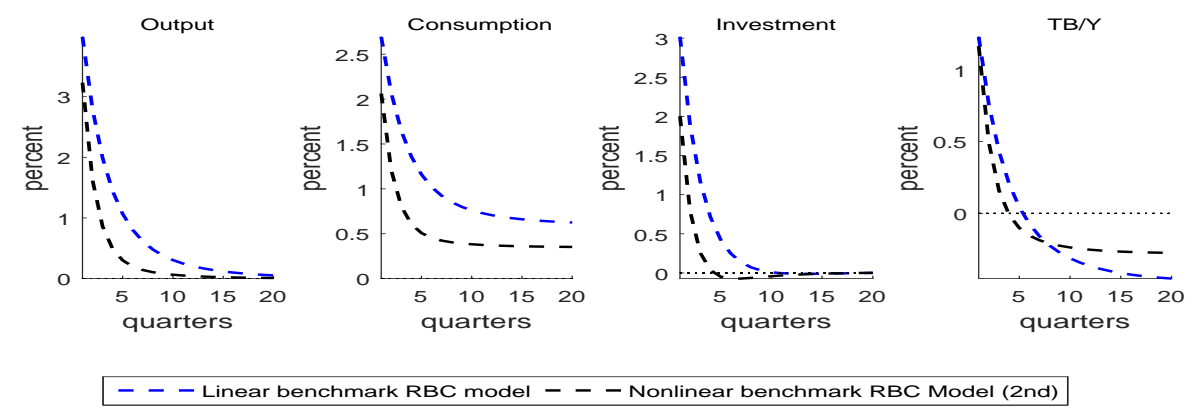

Figure 3.3: Impulse responses to temporary prod. shocks
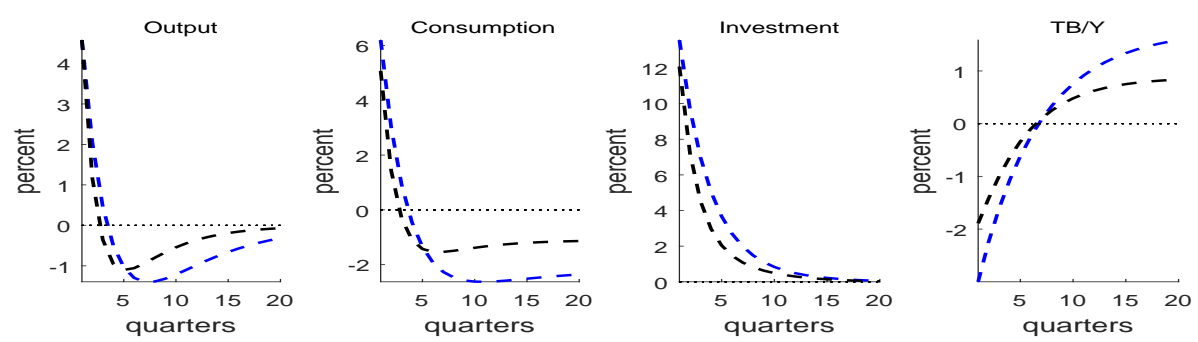

- - - Linear benchmark RBC model - - - Nonlinear benchmark RBC Model (2nd)

Figure 3.4: Impulse responses to permanent prod. shocks

put and trade balance to output ratio for both the linear and quadratic benchmark RBC models. However, Figure 3.4 shows that the response of the trade balance to output ratio to a nonstationary productivity shock is markedly different. Following a one-standard-deviation permanent productivity shock, the trade balance to output ratio decreases by $3.0 \%$ and $2.0 \%$ in the linear and quadratic benchmark RBC models, respectively. This difference in the response of the trade balance to output ratio arises from the different response of consumption. As a nonstationary productivity shock implies a permanent increase in income, consumption will respond more to such shocks due to the permanent income hypothesis. On the other hand, a transitory productivity shock induces saving in anticipation of lower income in the future as productivity mean-reverts. This leads to a less consumption response than output. 

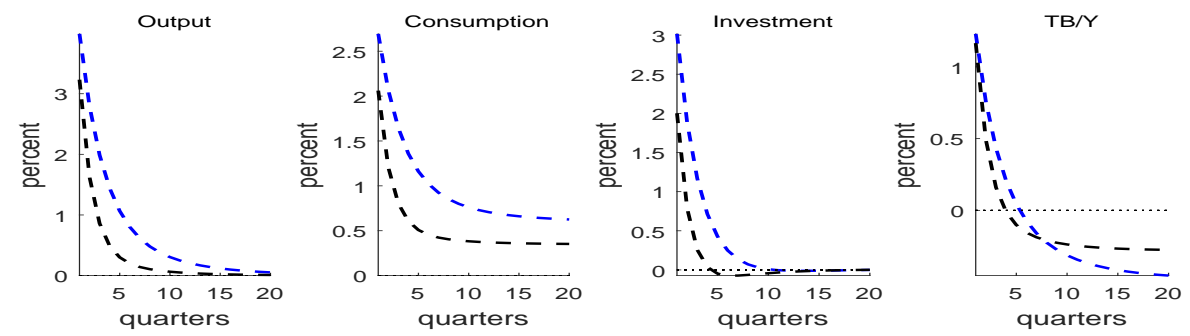

- - - Linear benchmark RBC model - - - Nonlinear benchmark RBC Model (2nd)

Figure 3.5: Impulse responses to temporary prod. shocks
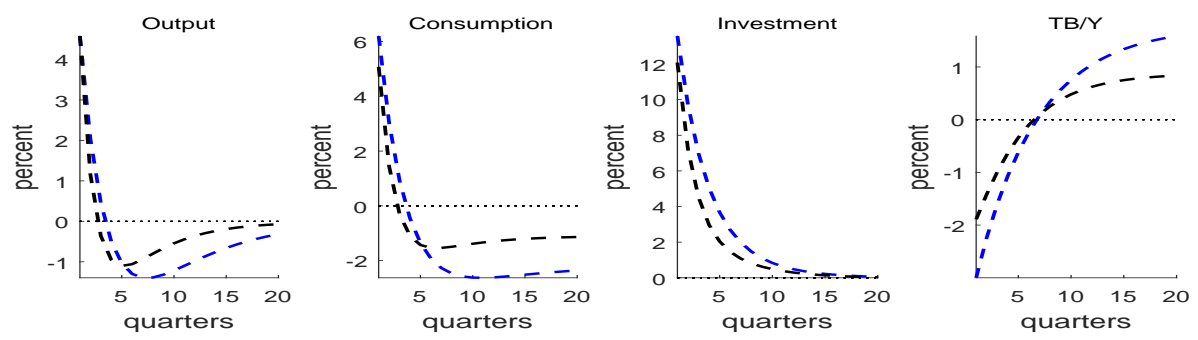

- - - Linear benchmark RBC model - - - Nonlinear benchmark RBC Model (2nd)

Figure 3.6: Impulse responses to permanent prod. shocks

\section{Impulse responses in a $\mathrm{RBC}$ model with financial frictions}

Productivity shocks (one-standard-deviation shocks): Figure 3.5 and Figure 3.6 show impulse responses to stationary and nonstationary productivity shocks, obtained from the linear and quadratic RBC models with financial frictions. A stationary shock in the augmented RBC model with financial frictions leads to a steeper consumption response which allows consumption to be more volatile than the benchmark RBC model. Also, it leads investment to respond more strongly. These results in a sharp fall in the trade balance to output ratio below the trend. Contrary to the linear model with financial frictions, the quadratic model with financial frictions shows that nonstationary productivity shocks have larger impacts on output, consumption, and investment than stationary productivity shocks. In addition, nonstationary productivity shocks in the quadratic model with financial frictions generates more persistent impact on output, consumption, and investment, reflecting increased 

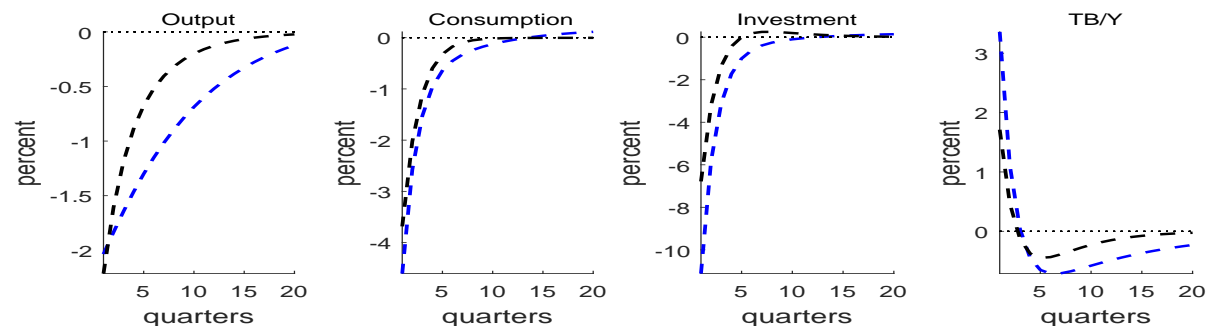

- - - Linear financial friction model - - - Nonlinear financial friction Model (2nd)

Figure 3.7: Country premium shocks
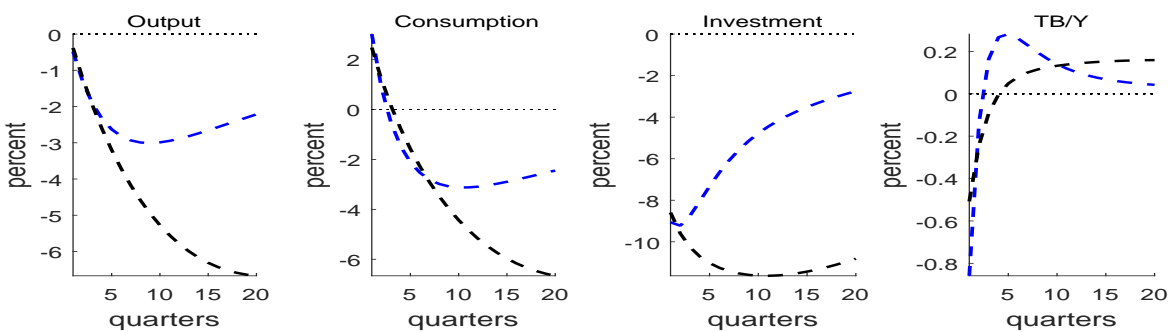

- - - Linear financial friction model - - - Nonlinear financial friction Model (2nd)

Figure 3.8: Preference shocks
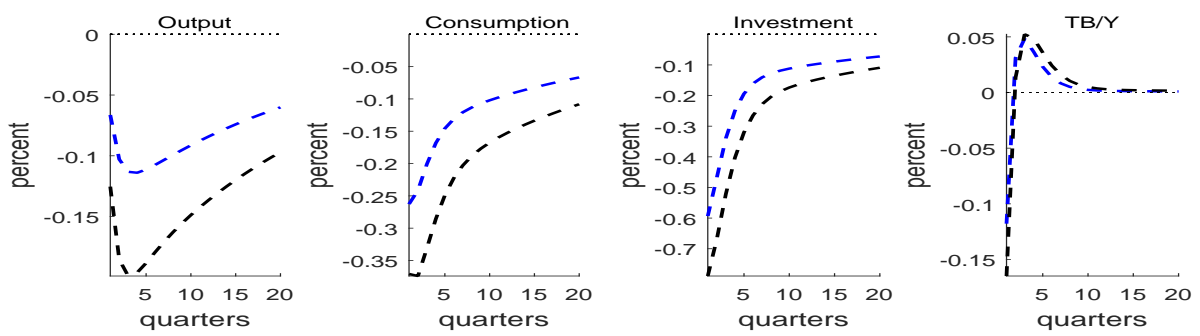

- - - Linear financial friction model --- Nonlinear financial friction Model (2nd)

Figure 3.9: Spending shocks

uncertainty about future permanent income. The persistent falls in consumption and investment in the quadratic model with financial frictions reflect a household's precautionary savings motive and a negative real-options effect on firm's investment decision.

Country premium and other shocks (one-standard-deviation shocks): Figure 3.7, Figure 3.8, and Figure 3.9 show impulse responses to country premium, spending, and preference shocks, respectively. A one-standard-deviation shock in the 
country premium raises the interest rate at which the country borrows from world financial markets. Elevated interest rates curb consumption and investment more strongly than output, thereby inducing the trade balance to become positive. Investment and the trade balance to output ratio are to a large extent driven by innovations in the country interest rate. Figure 3.8 shows that a preference shock increases consumption, and hence reduces the trade balance to output ratio. The decrease in the trade balance to output ratio cause the external debt to increase, leading to an increase in the interest rate. This further reduces consumption and investment. In addition, private consumption is affected in equal parts by a domestic preference shock and a stationary productivity shock in the linear model, but it is explained in equal parts by a domestic preference shock and a nonstationary productivity shock in the quadratic model. Finally, domestic spending shocks are estimated to have a negligible role in explaining business cycles in the linear and quadratic models. The domestic spending shock that can be interpreted as a government purchase shock tends to crowd out consumption and investment. The shock reduces the trade balance to output ratio, and hence increases the level of the external debt. The financial frictions lead to an increase in the interest rate, further reducing consumption and investment.

\subsection{Conclusion}

We have presented the results of an empirical analysis of the non-linear features of standard RBC models for the small open economy. With several advantages posed by the quadratic approximation of the original nonlinear model, a few main results emerge which we could not obtain from the linearized model. First, the quadratic RBC model with financial frictions does a good job at identifying the parameters of the nonstationary productivity shock process. Second, the observed data favor 
the quadratic benchmark RBC and financial-friction models over the linear models. Third, the quadratic RBC model with financial frictions does a better job at capturing serial correlations of the observed data than the linear model with financial frictions. Fourth, contrary to the linear model with financial frictions, a nonstationary productivity shock in the quadratic model plays an important role in explaining Argentine economic fluctuations. However, both the linear and quadratic RBC models do a poor job at explaining business cycles in Argentina. The models do not capture the volatility of the observed trade balance to output ratio. 


\section{Appendix A}

\section{Appendix to Chapter 1}

\section{A.1 Central Difference Kalman filter}

We define the following matrices which make it possible to match the first-order terms,

$$
\begin{gathered}
S_{x x, t \mid t-1}^{(1)}=\left\{\left(h_{l}\left(\hat{\chi}_{i, t-1}^{x+}, \hat{\chi}_{0, t}^{\eta} ; \theta\right)-h_{l}\left(\hat{\chi}_{i, t-1}^{x-}, \hat{\chi}_{0, t}^{\eta} ; \theta\right)\right) / 2 h\right\}, \\
S_{x \eta, t \mid t-1}^{(1)}=\left\{\left(h_{l}\left(\hat{\chi}_{0, t-1}^{x}, \hat{\chi}_{i, t}^{\eta+} ; \theta\right)-h_{l}\left(\hat{\chi}_{0, t-1}^{x}, \hat{\chi}_{i, t}^{\eta-} ; \theta\right)\right) / 2 h\right\}, \\
S_{y x, t \mid t-1}^{(1)}=\left\{\left(\left(g_{l}\left(\bar{\chi}_{i, t}^{x+} ; \theta\right)-\left(g_{l}\left(\bar{\chi}_{i, t}^{x-} ; \theta\right)\right) / 2 h\right\},\right.\right.
\end{gathered}
$$

and the second-order terms in a Taylor series expansion of our nonlinear state space equations (1.2) and (1.3) (Andreasen, 2013),

$$
S_{x x, t \mid t-1}^{(2)}=\left\{\frac{\sqrt{h^{2}-1}}{2 h^{2}}\left(h_{l}\left(\hat{\chi}_{i, t-1}^{x+}, \hat{\chi}_{0, t}^{\eta} ; \theta\right)+h_{l}\left(\hat{\chi}_{i, t-1}^{x-}, \hat{\chi}_{0, t}^{\eta} ; \theta\right)-2 h_{l}\left(\hat{\chi}_{0, t-1}^{x}, \hat{\chi}_{0, t}^{\eta} ; \theta\right)\right)\right\}
$$




$$
\begin{gathered}
S_{x \eta, t \mid t-1}^{(2)}=\left\{\frac{\sqrt{h^{2}-1}}{2 h^{2}}\left(h_{l}\left(\hat{\chi}_{0, t-1}^{x}, \hat{\chi}_{i, t}^{\eta+} ; \theta\right)+h_{l}\left(\hat{\chi}_{0, t-1}^{x}, \hat{\chi}_{i, t}^{\eta-} ; \theta\right)-2 h_{l}\left(\hat{\chi}_{0, t-1}^{x}, \hat{\chi}_{0, t}^{\eta} ; \theta\right)\right)\right\}, \\
S_{y x, t \mid t-1}^{(2)}=\left\{\frac{\sqrt{h^{2}-1}}{2 h^{2}}\left(g_{l}\left(\bar{\chi}_{i, t}^{x+} ; \theta\right)+g_{l}\left(\bar{\chi}_{i, t}^{x-} ; \theta\right)-2 g_{l}\left(\bar{\chi}_{0, t}^{x} ; \theta\right)\right)\right\},
\end{gathered}
$$

where $g_{l}(\cdot)$ and $h_{l}(\cdot)$ are $l$ th equation of $g(\cdot)$ and $h(\cdot)$, respectively. The sigma-points are used to evaluate the mean and covariance matrix of prediction density:

$$
\begin{gathered}
\hat{x}_{t \mid t-1}=\frac{h^{2}-n_{x}-n_{\eta}}{h^{2}} h\left(\hat{\chi}_{0, t-1}^{x}, \hat{\chi}_{0, t}^{\eta} ; \theta\right)+\frac{1}{2 h^{2}} \sum_{i=1}^{n_{x}} h\left(\hat{\chi}_{i, t-1}^{x+}, \hat{\chi}_{0, t}^{\eta} ; \theta\right)+h\left(\hat{\chi}_{i, t-1}^{x-}, \hat{\chi}_{0, t}^{\eta} ; \theta\right) \\
+\frac{1}{2 h^{2}} \sum_{i=1}^{n_{\eta}} h\left(\hat{\chi}_{0, t-1}^{x}, \hat{\chi}_{i, t}^{\eta+} ; \theta\right)+h\left(\hat{\chi}_{0, t-1}^{x}, \hat{\chi}_{i, t}^{\eta-} ; \theta\right) \\
S_{t \mid t-1}^{x}=\Phi\left(\left[S_{x x, t \mid t-1}^{(1)} \quad S_{x \eta, t \mid t-1}^{(1)} S_{x x, t \mid t-1}^{(2)} \quad S_{x \eta, t \mid t-1}^{(2)}\right]\right) \\
\hat{y}_{t \mid t-1}=\frac{h^{2}-n_{x}}{h^{2}} g\left(\bar{\chi}_{0, t}^{x}\right)+\frac{1}{2 h^{2}} \sum_{i=1}^{n_{x}}\left(g\left(\bar{\chi}_{i, t}^{x+} ; \theta\right)+g\left(\bar{\chi}_{i, t}^{x-} ; \theta\right)\right)
\end{gathered}
$$

The above prediction step gives the Gaussian-based prediction density $p\left(x_{t} \mid y_{1: t-1} ; \theta\right) \approx$ $N\left(\hat{x}_{t \mid t-1}, P_{t \mid t-1}^{x}\right)$ where $P_{t \mid t-1}^{x}=S_{t \mid t-1}^{x} S_{t \mid t-1}^{x}{ }^{\prime}$. The predictions are then updated using the standard Kalman filter updating rule:

$$
\begin{aligned}
& S_{t \mid t-1}^{y}=\Phi\left(\left[S_{y x, t \mid t-1}^{(1)} \quad S_{v} \quad S_{y x, t \mid t-1}^{(2)}\right]\right), \\
& K_{t}=S_{t \mid t-1}^{x} S_{y x, t \mid t-1}^{(1)^{\prime}}\left[S_{t \mid t-1}^{y} S_{t \mid t-1}^{y}\right]^{-1}, \\
& \hat{x}_{t \mid t}=\hat{x}_{t \mid t-1}+K_{t}\left(y_{t}-\hat{y}_{t \mid t-1}\right), \\
& S_{t \mid t}^{x}=\Phi\left(\left[S_{t \mid t-1}^{x}-K_{t} S_{y x, t \mid t-1}^{(1)} \quad K_{t} S_{v} \quad K_{t} S_{y x, t \mid t-1}^{(2)}\right]\right),
\end{aligned}
$$

where $S_{v}$ is the upper triangular Cholesky factorization of $\mathrm{R}_{v}$. The updating step gives $p\left(x_{t} \mid y_{1: t} ; \theta\right) \approx N\left(\hat{x}_{t \mid t}, P_{t \mid t}^{x}\right)$ where $P_{t \mid t}^{x}=S_{t \mid t}^{x} S_{t \mid t}^{x}$. Finally we can get the following 
conditional marginal likelihood,

$$
\hat{p}^{\mathrm{CDKF}}\left(y_{t} \mid y_{1: t-1} ; \theta\right)=N\left(\hat{y}_{t \mid t-1}, P_{t \mid t-1}^{y}\right),
$$

where $P_{t \mid t-1}^{y}=S_{t \mid t-1}^{y} S_{t \mid t-1}^{y}{ }^{\prime}$. 


\section{A.2 Binomial Gaussian mixture}

Following Raitoharju et al. (2015), the Binomial Gaussian mixture splits a normal distributed mixture component of the prediction and filtering density into smaller ones using weights and transformed locations from the binomial distribution. The probability density function of the standardized binomial distribution with the smallest error is expressed using Dirac delta notation as

$$
P_{B, m}(x)=\sum_{k=1}^{m}\left(\begin{array}{c}
m-1 \\
k-1
\end{array}\right)\left(\frac{1}{2}\right)^{m-1} \delta\left(x-\frac{2 k-m-1}{\sqrt{m-1}}\right),
$$

where $m$ denotes the number of the composite components. According to Berry-Essen theorem, the $P_{B, m}$ converges to the standard normal distribution as $m$ increases. Based on this well-known property, the Binomial Gaussian mixture is constructed using a mixture of standard normal distributions of which component means, covariance matrices, and weights are chosen based on a scaled binomial distribution. A proper affine transformation allows the mixture product to preserve the mean and covariance of the original Gaussian distribution.

A Binomial Gaussian mixture with $m_{\text {total }}=\prod_{i=1}^{n} m_{i}$ components has the following representation

$$
p_{\text {BinoGM }}(x)=\sum_{l=l}^{m_{\text {total }}} w_{l} p_{N}\left(x \mid \mu_{l}, P\right)
$$

where

$$
\begin{array}{r}
w_{l}=\prod_{i=1}^{n}\left(\begin{array}{c}
m_{i}-1 \\
C_{l, i}-1
\end{array}\right)\left(\frac{1}{2}\right)^{m_{i}-1}, \\
\mu_{l}=T\left[\begin{array}{c}
\sigma_{1} \frac{2 C_{l, 1}-m_{1}-1}{\sqrt{m_{1}-1}} \\
\sigma_{2} \frac{2 C_{l, 2}-m_{2}-1}{\sqrt{m_{2}-1}} \\
\vdots \\
\sigma_{n} \frac{2 C_{l, n}-m_{n}-1}{\sqrt{m_{n}-1}}
\end{array}\right]+\mu,
\end{array}
$$




$$
P=T T^{\prime}
$$

$m_{i}$ is the number of components in $i$ th binomial distribution, $\sigma_{i}$ is the variance in $i$ th binomial distribution, $T$ is the operator for the affine transformation, and $C$ is the Cartesian product

$$
C=\left\{1, \ldots, m_{1}\right\} \times\left\{1, \ldots, m_{2}\right\} \times \cdots \times\left\{1, \ldots, m_{n}\right\}
$$

Notation $C_{l, i}$ is the $i$ th component of the $l$ th combination. If $m_{k}=1$, the term $\frac{2 C_{l, k}-m_{k}-1}{\sqrt{m_{k}-1}}$ is set to 0 .

Raitoharju et al. (2015) use the notation to define a random variable $\boldsymbol{x}_{\boldsymbol{B i n o G M}}$ distributed according to the above Binomial Gaussian mixture.

$$
\boldsymbol{x}_{\text {BinoGM }} \sim \operatorname{BinoGM}\left(\mu, T, \Sigma, m_{1}, \ldots, m_{n}\right) \text { where } \Sigma=\operatorname{diag}\left(\sigma_{1}^{2}, \ldots, \sigma_{n}^{2}\right) .
$$

They show that

$$
\begin{gathered}
E\left(\boldsymbol{x}_{\text {BinoGM }}\right)=\mu, \\
\operatorname{cov}\left(\boldsymbol{x}_{\text {BinoGM }}\right)=P_{0}=T(\Sigma+I) T^{\prime}, \\
\lim _{m_{1}, \ldots, m_{n} \rightarrow \infty} \operatorname{Bino} G M\left(\mu, T, \Sigma, m_{1}, \ldots, m_{n}\right)=N\left(\mu, T(\Sigma+I) T^{\prime}\right) .
\end{gathered}
$$

The equation (A.21) implies that the Binomial Gaussian mixture converges to the original Gaussian distribution in the limit. In splitting a mixture component, the parameters are chosen such that the mean and covariance are preserved. The mean is preserved by choosing $\mu$ in (A.18) to be the mean of the mixture component. If the component covariance matrix is $P_{0}$ and it is split into smaller components that have covariance $P$, matrices $T$ and $\Sigma$ have to be chosen so that

$$
T T^{\prime}=P
$$




$$
T \Sigma T^{\prime}+P=P_{0}
$$

The parameters for the Binomial Gaussian mixture are chosen such that it is a good approximation of the original mixture component, the nonlinearity is below a predetermined threshold $\eta_{\text {limit }}$, two equally weighted components that are next to each other produce a unimodal pdf, and the number of components is minimized (see Appendix in Raitoharju et al., 2015). Under these conditions, the matrix $T$ is chosen as

$$
T=L\left(P_{0}\right) V \operatorname{diag}\left[\frac{1}{\sqrt{\sigma_{1}^{2}+1}}, \frac{1}{\sqrt{\sigma_{2}^{2}+1}}, \ldots, \frac{1}{\sqrt{\sigma_{n}^{2}+1}}\right],
$$

where $L\left(P_{0}\right)$ is the square root matrix of $P_{0}, \sigma_{i}^{2}=m_{i}-1, \frac{\lambda_{i}^{2}}{m_{i}^{2}}=\frac{\lambda_{j}^{2}}{m_{j}^{2}}$ with the conditions that $m_{i} \geq 1, \frac{1}{R} \sum_{i=1}^{n} \frac{\lambda_{i}^{2}}{m_{i}^{2}}$ is not larger than a predetermined nonlinearity threshold $\eta_{\text {limit }}$, and $\lambda_{i}$ is the $i$ th eigenvalue in the eigendecomposition

$$
V \Lambda V^{\prime}=\frac{Q^{l}}{h^{2}}
$$

where $l$ indicates the $l$ th measurement equation, $h$ is a scaling parameter that determines the spread of the sigma-points around predictive or filtered mean, and $Q^{l}$ is defined as follows:

$Q_{[c, d]}^{l}= \begin{cases}g_{l}\left(\chi_{t \mid t-1}^{a}+h \mathrm{~S}_{i, t \mid t-1}^{a}\right)+g_{l}\left(\chi_{t \mid t-1}^{a}-h \mathrm{~S}_{i, t \mid t-1}^{a}\right)-2 g_{l}\left(\chi_{t \mid t-1}^{a}\right) & c=d \\ \frac{1}{2}\left[g_{l}\left(\chi_{t \mid t-1}^{a}+h \mathrm{~S}_{i, t \mid t-1}^{a}+h \mathrm{~S}_{i, t \mid t-1}^{a}\right)+g_{l}\left(\chi_{t \mid t-1}^{a}-h \mathrm{~S}_{i, t \mid t-1}^{a}-h \mathrm{~S}_{i, t \mid t-1}^{a}\right)-\right. & \\ \left.2 g_{l}\left(\chi_{t \mid t-1}^{a}\right)-Q_{[c, c]}^{l}-Q_{[d, d]}^{l}\right] & c \neq d,\end{cases}$ where the function $g$ describes the measurement equation. All of the notations come from the definition of the sigma-points discussed in the equation (1.7) for the CDKF. 


\section{A.3 Proof}

Proof of Corollary 1 We show that the acceptance rate of the pseudo-marginal MH algorithm converges to that of the ideal marginal $\mathrm{MH}$ algorithm:

$$
\hat{r}^{\mathrm{GMF}}\left(\theta^{*} \mid \theta\right)=\frac{\hat{p}^{\mathrm{GMF}}\left(y_{1: T} \mid \theta^{*}\right) p\left(\theta^{*}\right) q\left(\theta \mid \theta^{*}\right)}{\hat{p}^{\mathrm{GMF}}\left(y_{1: T} \mid \theta\right) p(\theta) q\left(\theta^{*} \mid \theta\right)} \underset{J \rightarrow \infty}{\longrightarrow} r\left(\theta^{*} \mid \theta\right)=\frac{p\left(y_{1: T} \mid \theta^{*}\right) p\left(\theta^{*}\right) q\left(\theta \mid \theta^{*}\right)}{p\left(y_{1: T} \mid \theta\right) p(\theta) q\left(\theta^{*} \mid \theta\right)},
$$

where $J$ is the total number of Gaussian mixture components of the marginal likelihood. It is a direct result of Theorem 12 of Ali-Löytty (2008) which is about the convergence of the GMF. Under the assumption that the Gaussian mixture prediction and filtered density weakly converge to the true densities, the marginal likelihood implied by the GMF weakly converges to the true marginal likelihood as the number of mixture component $J$ goes to infinity:

$$
\begin{aligned}
\hat{p}^{\mathrm{GMF}}\left(y_{t} \mid y_{1: t-1} ; \theta\right) & =\sum_{l^{\prime}=1}^{L^{\prime}} \sum_{n=1}^{N} w_{t}^{\left[l^{\prime}\right]} \gamma_{t}^{[n]} N\left(\hat{y}_{t \mid t-1}^{\left[l^{\prime} \times n\right]}, P_{t \mid t-1}^{y,\left[l^{\prime} \times n\right]}\right)=\sum_{i=1}^{J} l_{t}^{[i]} N\left(\hat{y}_{t \mid t-1}^{[i]}, P_{t \mid t-1}^{y,[i]}\right) \\
& \underset{J \rightarrow \infty}{\longrightarrow} p\left(y_{t} \mid y_{1: t-1} ; \theta\right),
\end{aligned}
$$

where $J=L^{\prime} N$ is the total number of Gaussian mixture components of the marginal

likelihood and $l_{t}^{[i]}=w_{t}^{\left[l^{\prime}\right]} \gamma_{t}^{[n]}$. As a result, the realizations of GMF with PM-MH converge to the true and stationary density.

Proof of Corollary 2 Assumption 2 ensures that the drift function $V$ satisfies

$$
\left.\| \hat{\mathbf{P}}^{G M F, i}\left(A \mid \theta_{0}\right)-\pi(A)\right) \| \leq R V\left(\theta_{0}\right) \rho^{i} \quad \text { for any } \theta_{0} \in \Theta, \text { as } J \rightarrow \infty
$$

where $V(\cdot)$ denotes a drift function, satisfying (1.41), $\rho<1$, and $R<\infty$. $J$ is the total number of mixture components of the marginal likelihood and $\Theta$ is the support set of the true posterior $p\left(\theta \mid y_{1: T}\right)$. The proof is the direct consequences of the Gaus- 
sian mixture approximation in Corollary 1 and Theorem 3.1 in Jarner and Hansen (2000).

Proof of Corollary 3 Under regularity conditions of Theorem 2 in Müller (2013), the asymptotic posterior normality ( $a$ ) based on the Gaussian-based filters corresponds to Theorem 2 in Müller (2013). The argument (b) is based on Corollary 1 describing that the realizations of GMF with PM-MH converge to the true and stationary density when the likelihood constructed by infinite Gaussian mixtures weakly converges to the true likelihood. 


\section{A.4 Second-order Approximation for Calibrated Parameters}

\section{Benchmark Case:}

$$
\begin{aligned}
& G_{x}=\left[\begin{array}{ccc}
0.55989 & 0.37427 & -0.8481 \\
-0.8026 & 4.08271 & 4.17808 \\
-0.1348 & 0.367 & 0.4974
\end{array}\right] \quad H_{x}=\left[\begin{array}{ccc}
0.95494 & 0.10207 & 0.12945 \\
8.99 E-17 & 0.95 & 0 \\
2.87 E-17 & 1.25 E-17 & 0.72
\end{array}\right] \\
& G_{x x}=\left[\begin{array}{ccccccccc}
0.02532 & -0.0501 & -0.0361 & -0.0501 & 0.06407 & 0.05009 & -0.0361 & 0.05009 & 0.04847 \\
-0.8661 & 2.26471 & 2.91292 & 2.26471 & -5.808 & -7.6907 & 2.91292 & -7.6907 & -10.328 \\
-0.0258 & 0.05932 & 0.06176 & 0.05932 & -0.119 & -0.1398 & 0.06176 & -0.1398 & -0.1781
\end{array}\right] \\
& H_{x x}=\left[\begin{array}{ccccccccc}
0.01795 & -0.0331 & -0.0409 & -0.0331 & 0.05795 & 0.06538 & -0.0409 & 0.06538 & 0.06859 \\
0 & 0 & 0 & 0 & 0 & 0 & 0 & 0 & 0 \\
0 & 0 & 0 & 0 & 0 & 0 & 0 & 0 & 0
\end{array}\right] \\
& g_{\sigma \sigma}=\left[\begin{array}{c}
0.00209 \\
-0.0103 \\
-0.0012
\end{array}\right] \quad h_{\sigma \sigma}=\left[\begin{array}{c}
-0.0003 \\
0 \\
0
\end{array}\right]
\end{aligned}
$$

Extremely Nonlinear Case:

$$
\begin{aligned}
& G_{x}=\left[\begin{array}{ccc}
0.49684 & 0.50058 & -0.757 \\
-1.6118 & 6.81266 & 8.81103 \\
-0.4907 & 1.46888 & 2.22657
\end{array}\right] \quad H_{x}=\left[\begin{array}{ccc}
0.93471 & 0.17032 & 0.24528 \\
-1.03 E-17 & 0.95 & -2.22 E-16 \\
-3.73 E-18 & 9.42 E-18 & 0.72
\end{array}\right] \\
& G_{x x}=\left[\begin{array}{ccccccccc}
0.03112 & -0.0737 & -0.0712 & -0.0737 & 0.12288 & 0.1261 & -0.0712 & 0.1261 & 0.15003 \\
-2.078 & 5.99445 & 8.61418 & 5.99445 & -16.805 & -24.773 & 8.61418 & -24.773 & -37.073 \\
-0.0968 & 0.23281 & 0.23351 & 0.23281 & -0.409 & -0.443 & 0.23351 & -0.443 & -0.5506
\end{array}\right] \\
& H_{x x}=\left[\begin{array}{ccccccccc}
0.03119 & -0.067 & -0.0969 & -0.067 & 0.14553 & 0.19527 & -0.0969 & 0.19527 & 0.24629 \\
0 & 0 & 0 & 0 & 0 & 0 & 0 & 0 & 0 \\
0 & 0 & 0 & 0 & 0 & 0 & 0 & 0 & 0
\end{array}\right] \\
& g_{\sigma \sigma}=\left[\begin{array}{c}
0.00312 \\
-0.0363 \\
-0.0092
\end{array}\right] \quad h_{\sigma \sigma}=\left[\begin{array}{c}
-0.0009 \\
0 \\
0
\end{array}\right]
\end{aligned}
$$




\section{Nearly Linear Case:}

$$
\begin{aligned}
& G_{x}=\left[\begin{array}{ccc}
0.58795 & 0.32678 & -0.8758 \\
-0.5386 & 3.26686 & 2.94909 \\
-0.0102 & 0.02658 & 0.03458
\end{array}\right] \quad H_{x}=\left[\begin{array}{ccc}
0.96154 & 0.08167 & 0.09873 \\
3.06 E-17 & 0.95 & 3.33 E-16 \\
9.29 E-18 & 4.74 E-18 & 0.72
\end{array}\right] \\
& G_{x x}=\left[\begin{array}{ccccccccc}
0.02472 & -0.0452 & -0.0291 & -0.0452 & 0.0553 & 0.0392 & -0.0291 & 0.0392 & 0.03611 \\
-0.5688 & 1.41942 & 1.74664 & 1.41942 & -3.4939 & -4.4351 & 1.74664 & -4.4351 & -5.7198 \\
-0.0011 & 0.00198 & 0.00141 & 0.00198 & -0.0027 & -0.0022 & 0.00141 & -0.0022 & -0.0023
\end{array}\right] \\
& H_{x x}=\left[\begin{array}{ccccccccc}
0.01463 & -0.0258 & -0.0304 & -0.0258 & 0.04272 & 0.04635 & -0.0304 & 0.04635 & 0.04707 \\
0 & 0 & 0 & 0 & 0 & 0 & 0 & 0 & 0 \\
0 & 0 & 0 & 0 & 0 & 0 & 0 & 0 & 0
\end{array}\right] \\
& g_{\sigma \sigma}=\left[\begin{array}{c}
1.98 E-05 \\
-6.67 E-05 \\
-7.82 E-07
\end{array}\right] \quad h_{\sigma \sigma}=\left[\begin{array}{c}
-1.67 E-06 \\
0 \\
0
\end{array}\right]
\end{aligned}
$$




\section{A.5 Benchmark with stochastic volatility}

Figure A.1: Posterior Distribution with $C A=0.1$
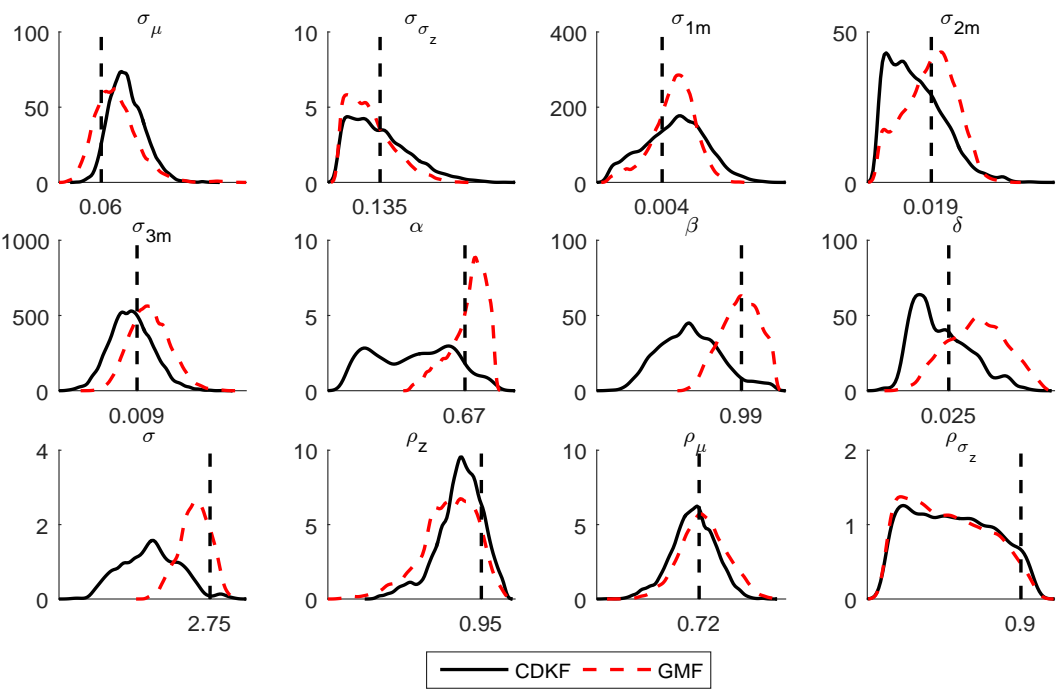

Notes. The vertical dashed line represents true parameter values.

Table A.1 Posterior Distribution with $C A=0.1$

\begin{tabular}{c|ccc|ccc}
\hline & \multicolumn{4}{|c}{ CDKF } & \multicolumn{3}{c}{ GMF } \\
\hline & Mean & S.D. & Prob. Interval & Mean & S.D. & Prob. Interval \\
\hline$\alpha$ & 0.5101 & 0.459 & $(0.000,0.999)$ & 0.6818 & 0.0568 & $(0.566,0.753)$ \\
$\beta$ & 0.9744 & 0.0838 & $(0.833,0.999)$ & 0.9896 & 0.0058 & $(0.979,0.999)$ \\
$\delta$ & 0.023 & 0.0496 & $(0.000,0.106)$ & 0.0324 & 0.0078 & $(0.019,0.045)$ \\
$\sigma$ & 2.1824 & 1.4584 & $(0.000,4.632)$ & 2.6039 & 0.1472 & $(2.341,2.830)$ \\
$\rho_{z}$ & 0.9046 & 0.0274 & $(0.858,0.950)$ & 0.8814 & 0.0588 & $(0.770,0.963)$ \\
$\rho_{\mu}$ & 0.7017 & 0.2641 & $(0.258,0.999)$ & 0.7342 & 0.0724 & $(0.616,0.857)$ \\
$\rho_{\sigma_{z}}$ & 0.4429 & 0.045 & $(0.367,0.518)$ & 0.4135 & 0.2643 & $(0.038,0.866)$ \\
$\sigma_{\mu}$ & 0.0683 & 0.0178 & $(0.038,0.098)$ & 0.0649 & 0.007 & $(0.055,0.077)$ \\
$\sigma_{\sigma_{z}}$ & 0.1348 & 0.0701 & $(0.017,0.252)$ & 0.1018 & 0.0746 & $(0.008,0.247)$ \\
$\sigma_{1 m}$ & 0.0049 & 0.0032 & $(0.000,0.010)$ & 0.0049 & 0.0016 & $(0.002,0.007)$ \\
$\sigma_{2 m}$ & 0.0137 & 0.0139 & $(0.000,0.037)$ & 0.0186 & 0.009 & $(0.003,0.033)$ \\
$\sigma_{3 m}$ & 0.0088 & 0.0003 & $(0.008,0.009)$ & 0.0095 & 0.0007 & $(0.008,0.011)$ \\
\hline
\end{tabular}

Effective Computing Time

\begin{tabular}{c|c|c}
\hline & 0.005 & 0.072 \\
\hline \multicolumn{2}{l}{ Root Mean Squared Errors (RMSEs) } \\
\hline & 1.5102 & 1.1534 \\
\hline \multicolumn{2}{c}{ Average Relative Biases (ARBs) } \\
\hline & 0.208 & 0.2054 \\
\hline
\end{tabular}




\section{A.6 Data Sources over 1991Q1-2015Q3}

1. Real Gross Domestic Product, BEA, NIPA table 1.1.6, line 1

2. Gross Domestic Product, BEA NIPA table 1.1.5, line 1

3. Personal Consumption Expenditure on Nondurable Goods, BEA, NIPA table 1.1 .5$, line 5

4. Personal Consumption Expenditure on Services, BEA NIPA table 1.1.5, line 6

5. Gross Private Domestic Investment, Fixed Investment, BEA NIPA table 1.1.5, line 8

6. GDP Deflator $=$ Gross Domestic Product/ Real Gross Domestic Product $=\# 2 / \# 1$

7. Real Consumption $=($ Personal Consumption Expenditure on Nondurable Goods + Services $) /$ GDP Deflator $=(\# 3+\# 4) / \# 6$

8. Real Investment $=($ Total private fixed investment + consumption expenditures on durable goods)/GDP Deflator $=\# 5 / \# 6$

9. Total hours is measured as total hours in the non-farm business sector, available from the BLS 


\section{Appendix B}

\section{Appendix to Chapter 2}

\section{B.1 Particle MCMC}

Let $p\left(\theta \mid y_{1: T}\right)$ be posterior probability density:

$$
p\left(\theta \mid y_{1: T}\right) \propto p\left(y_{1: T} \mid \theta\right) p(\theta)
$$

1. Draw $\theta_{0}$ from an initial distribution $p_{0}(\theta)$, set $S_{0}$ to be the lower triangular Cholesky factor of the initial covariance matrix $\Sigma_{0}$ and then, evaluate the initial approximate marginal likelihood $\hat{p}\left(y_{1: T} \mid \theta_{0}\right)$.

2. Sample a candidate using $q\left(\theta^{*} \mid \theta_{i-1}\right)\left(\theta^{*}=\theta_{i-1}+S_{i-1} \epsilon_{i}\right.$, where $\epsilon_{i} \sim N(0, I)$ in this study)

3. Evaluate the approximate marginal likelihood $\hat{p}\left(y_{1: T} \mid \theta^{*}\right)$ using the standard bootstrap particle filter. The bootstrap particle filter is implemented by the following steps:

(a) Draw initial particles $x_{0}^{[i]}$ from $p\left(x_{0}\right)$ and set $w\left(x_{0}^{[i]}\right)=1 / N$ for $i=1,2, \ldots, N$ 
(b) Draw a large number of samples of state variables $\left\{x_{t}^{[i]}\right\}_{i=1}^{N}$ from the following transition distribution,

$$
x_{t}^{[i]} \sim p\left(x_{t} \mid \hat{x}_{t-1}^{[i]} ; \theta\right) \text { for } i=1,2, \ldots, N
$$

(c) Evaluate the normalized importance weights

$$
w\left(x_{t}^{[i]}\right)=w\left(x_{t-1}^{[i]}\right) p\left(y_{t} \mid x_{t}^{[i]} ; \theta\right) \text { for } i=1,2, \ldots, N
$$

The conditional marginal likelihood is obtained from the following equation:

$$
\hat{p}^{\mathrm{PF}}\left(y_{t} \mid y_{1: t-1} ; \theta\right)=\sum_{i=1}^{N} w\left(x_{t}^{[i]}\right)
$$

(d) Resample with replacement from $\left\{x_{t}^{[i]}\right\}_{i=1}^{N}$ with probabilities $\tilde{w}_{t}^{[i]}=\frac{w\left(x_{t}^{[i]}\right)}{\sum_{i=1}^{N} w\left(x_{t}^{[i]}\right)}$ and set $w\left(x_{t}^{[i]}\right)=1 / N$ for $i=1,2, \ldots, N$. The resampled state variables are denoted by $\left\{\hat{x}_{t}^{[i]}\right\}_{i=1}^{N}$

A complete description can be found in Arulampalam, Maskell, Gordon, and Clapp (2002).

4. Calculate the acceptance probability,

$$
\hat{\alpha}\left(\theta^{*} \mid \theta_{i-1}\right)=\min \left\{1, \frac{\hat{p}\left(y_{1: T} \mid \theta^{*}\right) p\left(\theta^{*}\right) q\left(\theta_{i-1} \mid \theta^{*}\right)}{\hat{p}\left(y_{1: T} \mid \theta_{i-1}\right) p\left(\theta_{i-1}\right) q\left(\theta^{*} \mid \theta_{i-1}\right)}\right\}
$$

5. Sample a uniform random variable $u$ from the uniform distribution $\mathrm{U}(0,1)$

6. If $\mathbf{u} \leq \hat{\alpha}\left(\theta^{*} \mid \theta_{i-1}\right)$, set $\theta_{i}=\theta^{*}$. Otherwise set $\theta_{i}=\theta_{i-1}$.

7. (Optional) Adjust the covariance matrix of the Gaussian proposal satisfying the 
following equation,

$$
S_{i} S_{i}^{T}=S_{i-1}\left(\mathrm{I}+i^{-\gamma}\left(\hat{\alpha}^{k}\left(\theta^{*} \mid \theta_{i-1}\right)-\overline{\alpha_{*}}\right) \frac{\epsilon_{i} \epsilon_{i}^{T}}{\left\|\epsilon_{i}\right\|^{2}}\right) S_{i-1}^{T}
$$

where $\gamma \in(1 / 2,1]$ (Vihola, 2012)

8. Set $\mathrm{i}=\mathrm{i}+1$ and go to step 2

We adaptively adjust the proposal covariance matrix $S_{i}$ to achieve an acceptance rate around $30 \%$. 


\section{B.2 Markov-switching VAR}

We consider the following VAR model:

$$
\begin{gathered}
Y_{t}=c_{s_{t}}+\sum_{i=1}^{p} \phi_{s_{t} i} Y_{t-i}+\Sigma_{s_{t}}^{1 / 2} \varepsilon_{t}, \quad \varepsilon_{t} \sim N(0,1) . \\
P=\left[\begin{array}{ll}
p_{11} & p_{12} \\
p_{21} & p_{22}
\end{array}\right]
\end{gathered}
$$

where $s_{t}$ indexes regimes that follow 2-state Markov chain, $P$ denotes a transition probability matrix with components $p_{i j}=p\left(s_{t}=j \mid s_{t-1}=i\right)$. For the estimation, we use the following Gibbs sampler procedure with 50,000 iterations.

1. Sampling $S_{1: T}$ : Given previously sampled parameters, we draw the unobserved state variables $S_{1: T}$ from $f\left(S_{1: T} \mid Y_{1: T}\right)$ using a forward filtering and backward sampling procedure as described in Kim and Nelson (1999, Chapter 9). The forward filtering is based on Hamilton filter (1989).

2. Sampling $\Phi_{s}=\left[\phi_{s_{t} 1}, \ldots, \phi_{s_{t} p}, c_{s_{t}}\right]^{\prime}$ : We collect the VAR coefficients for regime $s$ into the matrix $B_{s}$, the LHS of equation (B.7) for regime $s$ into the matrix $Y_{s}$, and the RHS (lags and the intercept terms) of equation (B.7) into the matrix $X_{s}$. The equation (B.7) can be re-written as a system of multivariate regressions:

$$
Y_{s}=X_{s} \Phi_{s}+U_{s}
$$

We draw $\Phi_{s}$ from a Normal distribution (see equation (7) in Bańbura et al. (2010)). We impose prior beliefs on the autoregressive coefficients, the covari- 
ance matrix, and the intercept by adding the following dummy observations:

$Y_{d}=\left(\begin{array}{c}\operatorname{diag}\left(\delta_{1} \sigma_{1}, \ldots, \delta_{N} \sigma_{N}\right) / \lambda \\ 0_{N(p-1) \times N} \\ \operatorname{diag}\left(\sigma_{1}, \ldots, \sigma_{N}\right) \\ 0_{1 \times N}\end{array}\right) \quad X_{d}=\left(\begin{array}{cc}J_{p} \otimes \operatorname{diag}\left(\sigma_{1}, \ldots, \sigma_{N}\right) / \lambda & 0_{N P \times 1} \\ 0_{N \times N p} & 0_{N \times 1} \\ 0_{1 \times N p} & \varepsilon\end{array}\right)$

where $J_{p}=\operatorname{diag}(1,2, \ldots, p)$. We set the scale parameters $\sigma_{i}^{2}$ equal to the variance of a residual from a univariate autoregressive model of order $p$ for the variable $y_{i t}$. In this study, we set $\lambda$ and $\varepsilon$ to 0.1 and 0.0001 , respectively. We impose additional priors that constrain the sum of coefficients to improve forecasting performance. To achieve this goal, we add the following dummy observations:

$Y_{d}=\operatorname{diag}\left(\delta_{1} \mu_{1}, \ldots, \delta_{N} \mu_{N}\right) / \tau \quad X_{d}=\left(\left(1_{1 \times p}\right) \otimes \operatorname{diag}\left(\delta_{1} \mu_{1}, \ldots, \delta_{N} \mu_{N}\right) / \tau \quad 0_{N \times 1}\right)$

where the parameter $\mu_{i}$ captures the average level of variable $y_{i t}$ and $\tau=10 \lambda$.

3. Sampling $\Sigma_{s_{t}}$ : Given $c_{s_{t}}$ and $\phi_{s_{t} i}$, we can compute the residuals of the MS-VAR at each point in time and group all the residuals that pertain to a particular regime. Finally, we draw $\Sigma_{s_{t}}$ from an inverse Wishart distribution (see equation (7) in Bańbura et al. (2010)).

4. Sampling $P$ : Given $S_{1: T}$, the transition probabilities have a Dirichlet distribution as a conditional posterior. The posterior distribution is:

$$
p_{i j}=D\left(u_{i j}+v_{i j}\right)
$$

where $v_{i j}$ denotes the number of times regime $i$ is followed by regime $j . u_{i j}$ is the hyperparameters for the priors. $u_{11}$ is set to achieve $97 \%$ probability of 
staying in the regime 1 , but $u_{22}$ is set to achieve $75 \%$ probability of staying in the regime 2 . 


\section{B.3 Third-order Approximation}

The model solved using third-order approximation with pruning is given by the following state space representation

$$
\begin{aligned}
& \mathbf{Y}_{\mathbf{t}}=\mathbf{G}\left(\mathbf{X}_{\mathbf{t}}, \sigma\right)+\boldsymbol{\epsilon}_{\boldsymbol{t}} \\
& \mathbf{X}_{\mathbf{t}+\mathbf{1}}=\mathbf{H}\left(\mathbf{X}_{\mathbf{t}}, \sigma\right)+\sigma \boldsymbol{\eta}_{\boldsymbol{t}}
\end{aligned}
$$

where $\mathbf{X}_{\mathbf{t}}$ denotes a set of state variables that contain pre-determined endogenous and exogenous variables, $\mathbf{Y}_{\mathbf{t}}$ is a set of observable variables, $\boldsymbol{\epsilon}_{\boldsymbol{t}+\mathbf{1}} \sim \operatorname{i.i.dN}\left(0, \mathbf{R}_{\epsilon}\right)$, and $\boldsymbol{\eta}_{\boldsymbol{t}+\mathbf{1}} \sim$ i.i.dN $\left(0, \mathbf{R}_{\eta}\right)$.

These are re-expressed in a more specific way:

$$
\begin{aligned}
& \mathbf{X}_{\mathbf{t}+\mathbf{1}}=\left[\begin{array}{c}
\hat{\mathbf{x}}_{t+1}^{f} \\
\hat{\mathbf{x}}_{t+1}^{s} \\
\hat{\mathbf{x}}_{t+1}^{t h}
\end{array}\right] {\left[\begin{array}{c}
\mathbf{h}_{\mathbf{x}} \hat{\mathbf{x}}_{t}^{f}+\sigma \boldsymbol{\eta} \boldsymbol{\epsilon}_{t+1} \\
\mathbf{h}_{\mathbf{x}} \hat{\mathbf{x}}_{t}^{t h}+\mathbf{H}_{\mathbf{x x}}\left(\hat{\mathbf{x}}_{t}^{f} \otimes \hat{\mathbf{x}}_{t}^{s}\right)+\mathbf{H}_{\mathbf{x x x}}\left(\hat{\mathbf{x}}_{t}^{f} \otimes \hat{\mathbf{x}}_{t}^{f} \otimes \hat{\mathbf{x}}_{t}^{f}\right)+\frac{3}{6} \mathbf{h}_{\sigma \sigma \mathbf{x}} \sigma^{2} \hat{\mathbf{x}}_{t}^{f}+\frac{1}{6} \mathbf{h}_{\sigma \sigma \sigma} \sigma^{3}
\end{array}\right] } \\
& \mathbf{h}_{\mathbf{x}} \hat{\mathbf{x}}_{t}^{s}+\frac{1}{2} \mathbf{H}_{\mathbf{x x}}\left(\hat{\mathbf{x}}_{t}^{f} \otimes \hat{\mathbf{x}}_{t}^{f}\right)+\frac{1}{2} \mathbf{h}_{\sigma \sigma} \sigma^{2} \\
& \mathbf{Y}_{\mathbf{t}=} \mathbf{g}_{\mathbf{x}}\left(\hat{\mathbf{x}}_{t}^{f}+\hat{\mathbf{x}}_{t}^{s}+\hat{\mathbf{x}}_{t}^{t h}\right)+\frac{1}{2} \mathbf{G}_{\mathbf{x x}}\left(\left(\hat{\mathbf{x}}_{t}^{f} \otimes \hat{\mathbf{x}}_{t}^{f}\right)+2\left(\hat{\mathbf{x}}_{t}^{f} \otimes \hat{\mathbf{x}}_{t}^{s}\right)\right) \\
&+\frac{1}{6} \mathbf{G}_{\mathbf{x x x}}\left(\hat{\mathbf{x}}_{t}^{f} \otimes \hat{\mathbf{x}}_{t}^{f} \otimes \hat{\mathbf{x}}_{t}^{f}\right)+\frac{1}{2} \mathbf{g}_{\sigma \sigma} \sigma^{2}+\frac{3}{6} \mathbf{g}_{\sigma \sigma \mathbf{x}} \sigma^{2} \hat{\mathbf{x}}_{t}^{f}+\frac{1}{6} \mathbf{g}_{\sigma \sigma \sigma} \sigma^{3}
\end{aligned}
$$

where we eliminate the terms of higher-order effects than the third-order by using a pruning method (see, in particular Kim et al. (2008) and Andreasen, FernandezVillaverde, and Rubio-Ramirez (2017)). 


\section{B.4 Equilibrium Conditions}

\section{Budget constraint for lenders}

$$
\begin{aligned}
& c_{t}^{L}+\frac{k_{c, t}}{A_{k, t}}+k_{h, t}+k_{b, t}+q_{t}\left[h_{t}^{L}-\left(1-\delta_{h}\right) h_{t-1}^{L}+h_{t}^{r}-\left(1-\delta_{h}\right) h_{t-1}^{r}\right]+p_{l, t} l_{t}-b_{t}^{L} \\
& =\frac{w_{c, t}^{L} n_{c, t}^{L}}{X_{w c, t}^{L}}+\frac{w_{h, t}^{L} n_{h, t}^{L}}{X_{w h, t}^{L}}+\left(R_{c, t} z_{c, t}+\frac{1-\delta_{k c}}{A_{k, t}}\right) k_{c, t-1}+\left(R_{h, t} z_{h, t}+1-\delta_{k h}\right) k_{h, t-1} \\
& +p_{b, t} k_{b, t}-\frac{R_{t-1} b_{t-1}^{L}}{\pi_{t}}+\left(p_{l, t}+R_{l, t}\right) l_{t-1}+q_{t}^{r} h_{t-1}^{r}+D i v_{t}^{L}-\phi_{t}-\frac{a\left(z_{c, t}\right) k_{c, t-1}}{A_{k, t}}-a\left(z_{h, t}\right)
\end{aligned}
$$

where

$$
\begin{aligned}
& \operatorname{Div}_{t}^{L}=\left(1-\frac{1}{X_{t}}\right) Y_{t}+\left(1-\frac{1}{X_{w c, t}^{L}}\right) w_{c, t}^{L} n_{c, t}^{L}+\left(1-\frac{1}{X_{w h, t}^{L}}\right) w_{h, t}^{L} n_{h, t}^{L} \\
& \phi_{t}=\frac{\phi_{k c}}{2}\left(\frac{k_{c, t}}{k_{c, t-1}}-1\right)^{2} k_{c, t-1}+\frac{\phi_{k h}}{2}\left(\frac{k_{h, t}}{k_{h, t-1}}-1\right)^{2} k_{h, t-1} \\
& a\left(z_{c, t}\right)=R_{c}\left(\frac{1}{2} \bar{w} z_{c, t}^{2}+(1-\bar{w}) z_{c, t}+\left(\frac{\bar{w}}{2}-1\right)\right) \\
& a\left(z_{h, t}\right)=R_{h}\left(\frac{1}{2} \bar{w} z_{h, t}^{2}+(1-\bar{w}) z_{h, t}+\left(\frac{\bar{w}}{2}-1\right)\right)
\end{aligned}
$$

\section{Optimal conditions for lenders}

$$
\begin{gathered}
u_{c^{L}, t}^{L} q_{t}=\beta^{L} E_{t}\left[\frac{j_{t+1} z_{t+1}}{h_{t}^{L}}\right]+\beta^{L} E_{t}\left[u_{c^{L}, t+1}^{L} q_{t+1}(1-\delta)\right] \\
u_{c^{L}, t}^{L} q_{t}=\beta^{L} E_{t}\left[u_{c^{L}, t+1}^{L} q_{t+1}^{r}\right]+\beta^{L} E_{t}\left[u_{c^{L}, t+1}^{L} q_{t+1}(1-\delta)\right] \\
u_{c^{L}, t}^{L}=\beta^{L} E_{t}\left(\frac{u_{c^{L}, t+1}^{L} R_{t}}{\pi_{t+1}}\right) \\
u_{c^{L}, t}^{L}\left(\frac{1}{A_{k, t}}+\frac{\partial \phi_{c, t}}{\partial k_{c, t}}\right)=\beta^{L} E_{t} u_{c^{L}, t+1}^{L}\left(R_{c, t+1} z_{c, t+1}-\frac{a\left(z_{c, t+1}\right)+1-\delta_{k c}}{A_{k, t+1}}-\frac{\partial \phi_{c, t+1}}{\partial k_{c, t}}\right)
\end{gathered}
$$




$$
\begin{gathered}
u_{c^{L}, t}^{L}\left(1+\frac{\partial \phi_{h, t}}{\partial k_{h, t}}\right)=\beta^{L} E_{t} u_{c^{L}, t+1}^{L}\left(R_{h, t+1} z_{h, t+1}-a\left(z_{h, t+1}\right)+1-\delta_{k h}-\frac{\partial \phi_{h, t+1}}{\partial k_{h, t}}\right) \\
u_{c^{L}, t}^{L} w_{c, t}^{L}=u_{n c^{L}, t}^{L} X_{w c, t}^{L} \\
u_{c^{L}, t}^{L} w_{h, t}^{L}=u_{n h^{L}, t}^{L} X_{w h, t}^{L} \\
u_{c^{L}, t}^{L}\left(p_{b, t}-1\right)=0 \\
R_{c, t} A_{k, t}=a^{\prime}\left(z_{c, t}\right) \\
R_{h, t}=a^{\prime}\left(z_{h, t}\right) \\
u_{c^{L}, t}^{L} p_{l, t}=\beta^{L} E_{t} u_{c^{L}, t+1}^{L}\left(p_{l, t+1}+R_{l, t+1}\right)
\end{gathered}
$$

\section{Budget and borrowing constraint for borrowers}

$$
\begin{gathered}
c_{t}^{B}+q_{t}\left[h_{t}^{B}-\left(1-\delta_{h}\right) h_{t-1}^{B}\right]+q_{t}^{r} h_{t-1}^{r}-b_{t}^{B}=\frac{w_{c, t}^{B} n_{c, t}^{B}}{X_{w c, t}^{B}}+\frac{w_{h, t}^{B} n_{h, t}^{B}}{X_{w h, t}^{B}}-\frac{R_{t-1} b_{t-1}^{B}}{\pi_{t}}+D i v_{t}^{B} \\
b_{t}^{B}=m E_{t} \frac{q_{t+1} \pi_{t+1} h_{t}^{B}}{R_{t}}
\end{gathered}
$$

\section{Optimal conditions for borrowers}

$$
\begin{gathered}
u_{c^{B}, t}^{B} q_{t}^{r}=\beta^{B} E_{t}\left[\frac{j_{t+1} z_{t+1}}{h_{t}^{B}}\left[\kappa\left(h_{t}^{B}\right)^{\xi_{h}-1}+(1-\kappa)\left(h_{t}^{r}\right)^{\xi_{h}-1}\right]^{\frac{2-\xi_{h}}{\xi_{h}-1}}(1-\kappa)\left(h_{t}^{r}\right)^{\xi_{h}-2}\right] \\
u_{c^{B}, t}^{B} q_{t}=\beta^{B} E_{t}\left[\frac{j_{t+1} z_{t+1}}{h_{t}^{B}}\left[\kappa\left(h_{t}^{B}\right)^{\xi_{h}-1}+(1-\kappa)\left(h_{t}^{r}\right)^{\xi_{h}-1}\right]^{\frac{2-\xi_{h}}{\xi_{h}-1}} \kappa\left(h_{t}^{B}\right)^{\xi_{h}-2}\right] \\
+\beta^{B} E_{t}\left[u_{c^{B}, t+1}^{B} q_{t+1}(1-\delta)\right]+E_{t}\left[\frac{\lambda_{t} m q_{t+1} \pi_{t+1}}{R_{t}}\right] \\
u_{c^{B}, t}^{B}=\beta^{B} E_{t}\left(u_{c^{B}, t+1}^{B} \frac{R_{t}}{\pi_{t+1}}\right)+\lambda_{t} \\
u_{c^{B}, t}^{B} w_{c, t}^{B}=u_{n c^{B}, t}^{B} X_{w c, t}^{B}
\end{gathered}
$$




$$
\begin{gathered}
u_{c^{B}, t}^{B} w_{h, t}^{B}=u_{n h^{B}, t}^{B} X_{w h, t}^{B} \\
D i v_{t}^{B}=\left(1-\frac{1}{X_{w c, t}^{B}}\right) w_{c, t}^{B} n_{c, t}^{B}+\left(1-\frac{1}{X_{w h, t}^{B}}\right) w_{h, t}^{B} n_{h, t}^{B}
\end{gathered}
$$

\section{Intermediate goods firms}

$$
\begin{gathered}
Y_{t}=\left(A_{c, t}\left(\left(n_{c, t}^{L}\right)^{\alpha}\left(n_{c, t}^{B}\right)^{1-\alpha}\right)\right)^{1-\mu_{c}}\left(z_{c, t} k_{c, t-1}\right)^{\mu_{c}} \\
I H_{t}=\left(A_{h, t}\left(\left(n_{h, t}^{L}\right)^{\alpha}\left(n_{h, t}^{B}\right)^{1-\alpha}\right)\right)^{1-\mu_{h}-\mu_{l}-\mu_{b}}\left(z_{h, t} k_{h, t-1}\right)^{\mu_{h}} k_{b, t}^{\mu_{b}} l_{t-1}^{\mu_{l}} \\
\left(1-\mu_{c}\right) \alpha Y_{t}=X_{t} w_{c, t}^{L} n_{c, t}^{L} \\
\left(1-\mu_{c}\right)(1-\alpha) Y_{t}=X_{t} w_{c, t}^{B} n_{c, t}^{B} \\
\left(1-\mu_{h}-\mu_{b}-\mu_{l}\right) \alpha q_{t} I H_{t}=w_{h, t}^{L} n_{h, t}^{L} \\
\left(1-\mu_{h}-\mu_{b}-\mu_{l}\right)(1-\alpha) q_{t} I H_{t}=w_{h, t}^{B} n_{h, t}^{B} \\
\mu_{c} Y_{t}=X_{t} R_{c, t} z_{c, t} k_{c, t-1} \\
\mu_{h} q_{t} I H_{t}=R_{h, t} z_{h, t} k_{h, t-1} \\
\mu_{l} q_{t} I H_{t}=R_{l, t} l_{t-1} \\
\mu_{b} q_{t} I H_{t}=p_{b, t} k_{b, t}
\end{gathered}
$$

\section{Price Stickiness}

$$
\begin{aligned}
& \pi_{t}^{*}=\frac{P_{t}^{*}}{P_{t-1}}=\frac{\varepsilon_{p}}{\varepsilon_{p}-1} \pi_{t} \frac{x_{1 t}}{x_{2 t}} \\
& x_{1 t}=\lambda_{t} \frac{1}{X_{t}} Y_{t}+\beta^{L} \theta \pi_{t}^{-\iota_{\pi} \varepsilon_{p}} E_{t} \pi_{t+1}^{\varepsilon_{p}} x_{1 t+1} \\
& x_{2 t}=\lambda_{t} Y_{t}+\beta^{L} \theta \pi_{t}^{\iota_{\pi}\left(1-\varepsilon_{p}\right)} E_{t} \pi_{t+1}^{\varepsilon_{p}-1} x_{2 t+1} \\
& \pi_{t}^{1-\varepsilon_{p}}=\left(1-\theta_{\pi}\right) \pi_{t}^{*\left(1-\varepsilon_{p}\right)}+\theta_{\pi} \pi_{t-1}^{\iota_{\pi}\left(1-\varepsilon_{p}\right)}
\end{aligned}
$$




\section{Wage Stickiness}

$$
\begin{gathered}
w_{i, t}^{* j}=\frac{\varepsilon_{i, w}^{j}}{\varepsilon_{i, w}^{j}-1} \frac{f_{i, 1 t}^{j}}{f_{i, 2 t}^{j}} \\
f_{i, 1 t}^{j}=\lambda_{t}^{j} \frac{\left(w_{i, t}^{j}\right)^{1+\varepsilon_{i, w}^{j}}}{X_{i, t}^{j}} n_{i, t}^{j}+\beta^{j} \theta_{w, i} \pi_{t}^{-\iota_{w, i} \varepsilon_{i, w}^{j}} E_{t} \pi_{t+1}^{\varepsilon_{i, w}^{j}} f_{i, 1 t+1}^{j} \\
f_{i, 2 t}^{j}=\lambda_{t}^{j}\left(w_{i, t}^{j}\right)^{\varepsilon_{i, w}^{j}} n_{i, t}^{j}+\beta^{j} \theta_{w, i} \pi_{t}^{\iota_{w, i}\left(1-\varepsilon_{i, w}^{j}\right)} E_{t} \pi_{t+1}^{\varepsilon_{i, w}^{j}-1} f_{i, 2 t+1}^{j} \\
\left(w_{i, t}^{j}\right)^{1-\varepsilon_{i, w}}=\left(1-\theta_{w, i}\right)\left(w_{i, t}^{* j}\right)^{\left(1-\varepsilon_{i, w}^{j}\right)}+\theta_{w, i} \pi_{t-1}^{\iota_{w, i}\left(1-\varepsilon_{i, w}^{j}\right)} \pi_{t}^{\varepsilon_{i, w}^{j}-1}\left(w_{i, t-1}^{j}\right)^{\left(1-\varepsilon_{i, w}^{j}\right)}
\end{gathered}
$$

where $i=$ consumption sector (C) or housing sector (H) and $j=$ Borrowers (B) or lenders (L).

\section{Taylor-type rule}

$$
R_{t}=R_{t-1}^{r_{R}} \pi_{t}^{\left(1-r_{R}\right) r_{\pi}}\left(\frac{G D P_{t}}{G D P_{t-1}}\right)^{\left(1-r_{R}\right) r_{Y}} \overline{r r}^{1-r_{R}} \frac{\sigma_{R, t} u_{R, t}}{A_{s, t}} \quad \text { where } u_{R, t} \sim N(0,1)
$$

where $\overline{r r}$ is the steady state real interest-rate, $r_{R}, r_{\pi}, r_{Y}$ are the responses of the interest rate to changes in the lagged interest rate, inflation and GDP growth, respectively, the inflation target shock $\left(A_{s, t}\right)$ follows $\mathrm{AR}(1)$ process with stochastic volatility $\sigma_{s, t}$, and the shock to the monetary policy $u_{R, t}$ is assumed to have time-varying volatility $\sigma_{R, t}$.

\section{Market Clearing}

$$
\begin{gathered}
C_{t}+I K_{c, t} / A_{k, t}+I K_{h, t}+k_{b, t}=Y_{t}-\phi_{t} \\
H_{t}-\left(1-\delta_{h}\right) H_{t-1}=I H_{t} \\
b_{t}^{L}+b_{t}^{B}=0 \\
l_{t}=1
\end{gathered}
$$




\section{Shock processes}

Productivity in the consumption $\left(A_{c, t}\right)$, investment $\left(A_{k, t}\right)$, and housing sector $\left(A_{h, t}\right)$ follows:

$$
\ln \left(A_{i, t}\right)=\rho_{A, i} \ln \left(A_{i, t-1}\right)+\sigma_{i, t} u_{i, t} \quad \text { where } u_{i, t} \sim N(0,1)
$$

where $i=\{c, k, h\}$ and $u_{i, t}$ is the innovation. All of the stochastic volatility processes follow:

$$
\sigma_{j, t}=\left(1-\rho_{\sigma_{j}}\right) \sigma_{j}+\rho_{\sigma_{j}} \sigma_{j, t-1}+\eta_{j} v_{j, t} \quad \text { where } v_{j, t} \sim N(0,1)
$$

where $j=\{c, h, j, k, \tau, z, s, R\}, \sigma_{j, t}$ is the $j$-th stochastic volatility, $\sigma_{j}$ is the unconditional mean of $\sigma_{j, t}, \rho_{\sigma_{j}}$ is the level of persistence, and $\eta_{j}$ is the standard deviation of the volatility shock $v_{j, t}$. 


\section{B.5 Steady State}

$$
\begin{aligned}
& \bar{\pi}=1 \\
& R=\frac{1}{\beta^{L}} \\
& R_{k c}=\frac{1}{\beta^{L}}-\left(1-\delta_{k}\right) \\
& R_{k h}=\frac{1}{\beta^{L}}-\left(1-\delta_{k}\right) \\
& r=R-1 \\
& \zeta_{0}=\frac{k_{c}}{Y}=\left(\frac{\beta^{L} \mu_{c}}{1-\beta^{L}\left(1-\delta_{k c}\right)}\right) \frac{1}{X} \\
& \zeta_{1}=\frac{k_{h}}{q I}=\frac{\beta^{L} \mu_{h}}{1-\beta^{L}\left(1-\delta_{k h}\right)} \\
& \zeta_{2}=\frac{q h^{L}}{c^{L}}=\frac{\beta^{L} j}{1-\beta^{L}\left(1-\delta_{h}\right)} \\
& \zeta_{3}=\frac{q h^{B}}{c^{B}}=\frac{\beta^{B} j}{1-\beta^{B}\left(1-\delta_{h}\right)-\left(\beta^{L}-\beta^{B}\right) m} \frac{1}{1+\frac{1-\kappa}{\kappa}\left(\frac{h^{r}}{h^{B}}\right)^{\alpha-1}} \\
& \zeta_{4}=(R-1) \frac{m}{R} \\
& \zeta_{5}=\frac{q^{r}}{q}=1-\beta^{L}\left(1-\delta_{h}\right) \\
& \frac{h^{r}}{h^{B}}=\left(\frac{\zeta_{5}}{\left(1-\beta^{B}\left(1-\delta_{h}\right)-\left(\beta^{L}-\beta^{B}\right) m\right) \frac{1-\kappa}{\kappa}}\right)^{\frac{1}{\alpha-2}} \\
& \zeta_{6}=\frac{q^{r}}{q}=\left(1-\beta^{B}\left(1-\delta_{h}\right)-\left(\beta^{L}-\beta^{B}\right) m\right) \frac{1-\kappa}{\kappa}\left(\frac{h^{r}}{h^{B}}\right)^{\alpha-2} \\
& \zeta_{7}=\frac{q h^{r}}{c^{B}}=\frac{\beta^{B} j}{\zeta_{7}\left(1+\frac{\kappa}{1-\kappa}\left(\frac{h^{B}}{h^{r}}\right)^{\alpha-1}\right)} \\
& \lambda=\frac{1-\beta^{B} / \beta^{L}}{c^{B}}
\end{aligned}
$$




$$
\begin{aligned}
& f=\frac{X-1}{X} Y \\
& l=1 \\
& R_{l}=\mu_{l} q I \\
& b^{B}=m q h^{B} / R \\
& \delta_{h}^{\prime}=1-\frac{1-\delta_{h}}{1} \\
& \delta_{k}^{\prime}=1-\frac{1-\delta_{k}}{1} \\
& k_{c}=\zeta_{0} Y \\
& k_{h}=\zeta_{1} q I \\
& q h^{L}=\zeta_{2} c^{L} \\
& q h^{B}=\zeta_{3} c^{B} \\
& q h^{r}=\zeta_{5} c^{B} \\
& \delta_{h}^{\prime}\left(\zeta_{2} c^{L}+\zeta_{3} c^{B}+\zeta_{5} c^{B}\right)=q I \\
& c^{L}+c^{B}+\delta_{k}^{\prime}\left(\zeta_{0} Y+\zeta_{1} q I\right)=Y \\
& c^{L}+\delta_{h}^{\prime}\left[\zeta_{2} c^{L}+\zeta_{5} c^{B}\right]=f+r \zeta_{0} Y+r \zeta_{1} q I+\mu_{l} q I+\sum w n+\zeta_{4} \zeta_{3} c^{B}+\zeta_{5} c^{B}+d i v \\
& c^{B}+\delta_{h}^{\prime} \zeta_{3} c^{B}=\sum w n-\zeta_{4} \zeta_{3} c^{B}-\zeta_{5} c^{B}+d i v \\
& \left(1-\mu_{c}\right) \alpha \frac{Y}{X n_{c}^{L}}=w_{c}^{L} \\
& \left(1-\mu_{c}\right)(1-\alpha) \frac{Y}{X n_{c}^{B}}=w_{c}^{B} \\
& \left(1-\mu_{h}-\mu_{b}-\mu_{l}\right) \alpha \frac{q I}{n_{h}^{L}}=w_{h}^{L}
\end{aligned}
$$




$$
\begin{aligned}
& \left(1-\mu_{h}-\mu_{b}-\mu_{l}\right)(1-\alpha) \frac{q I}{n_{h}^{B}}=w_{h}^{B} \\
& w_{c}^{L} n_{c}^{L}+w_{h}^{L} n_{h}^{L}=\alpha\left(\left(1-\mu_{c}\right) \frac{Y}{X}+\left(1-\mu_{h}-\mu_{b}-\mu_{l}\right) q I\right) \\
& w_{c}^{B} n_{c}^{B}+w_{h}^{B} n_{h}^{B}=(1-\alpha)\left(\left(1-\mu_{c}\right) \frac{Y}{X}+\left(1-\mu_{h}-\mu_{b}-\mu_{l}\right) q I\right) \\
& \left(1+\delta_{h}^{\prime} \zeta_{2}\left(1-r \zeta_{1}-\mu_{l}-\alpha\left(1-\mu_{h}-\mu_{b}-\mu_{l}\right)\right)\right) c^{L} \\
& -\left(\left(r \zeta_{1}+\mu_{l}+\alpha\left(1-\mu_{h}-\mu_{b}-\mu_{l}\right)\right) \delta_{h}^{\prime} \zeta_{3}\right. \\
& \left.+\left(r \zeta_{1}+\mu_{l}+\alpha\left(1-\mu_{h}-\mu_{b}-\mu_{l}\right)-1\right) \delta_{h}^{\prime} \zeta_{5}+\zeta_{3} \zeta_{4}+\zeta_{5} \zeta_{7}\right) c^{B} \\
& =\left(\frac{X-1}{X}+r \zeta_{0} X+\alpha \frac{\left(1-\mu_{c}\right)}{X}\right) Y \\
& \left(1+\delta_{h}^{\prime} \zeta_{3}-(1-\alpha)\left(1-\mu_{h}-\mu_{b}-\mu_{l}\right) \delta_{h}^{\prime} \zeta_{3}\right. \\
& \left.-(1-\alpha)\left(1-\mu_{h}-\mu_{b}-\mu_{l}\right) \delta_{h}^{\prime} \zeta_{5}+\zeta_{4} \zeta_{3}+\zeta_{5} \zeta_{7}\right) c^{B} \\
& -(1-\alpha)\left(1-\mu_{h}-\mu_{b}-\mu_{l}\right) \delta_{h}^{\prime} \zeta_{2} c^{L} \\
& =(1-\alpha)\left(1-\mu_{c}\right) \frac{1}{X} Y \\
& \frac{c^{L}}{Y}=\frac{\chi_{3} \chi_{4}+\chi_{2} \chi_{6}}{\chi_{1} \chi_{4}-\chi_{2} \chi_{5}} \\
& \frac{c^{B}}{Y}=\frac{\chi_{1} \chi_{6}+\chi_{3} \chi_{5}}{\chi_{1} \chi_{4}-\chi_{2} \chi_{5}} \\
& \frac{q I}{Y}=\delta_{h}^{\prime}\left(\zeta_{2} c^{L}+\zeta_{3} c^{B}\right) \\
& \chi_{1}=1+\delta_{h}^{\prime} \zeta_{2}\left(1-r \zeta_{1}-\mu_{l}-\alpha\left(1-\mu_{h}-\mu_{b}-\mu_{l}\right)\right) \\
& \chi_{2}=\left(r \zeta_{1}+\mu_{l}+\alpha\left(1-\mu_{h}-\mu_{b}-\mu_{l}\right)\right) \delta_{h}^{\prime} \zeta_{3} \\
& +\left(r \zeta_{1}+\mu_{l}+\alpha\left(1-\mu_{h}-\mu_{b}-\mu_{l}\right)-1\right) \delta_{h}^{\prime} \zeta_{5}+\zeta_{3} \zeta_{4}+\zeta_{5} \zeta_{7} \\
& \chi_{3}=\frac{X-1}{X}+r \zeta_{0} X+\alpha \frac{\left(1-\mu_{c}\right)}{X} \\
& \chi_{4}=1+\delta_{h}^{\prime} \zeta_{3}-(1-\alpha)\left(1-\mu_{h}-\mu_{b}-\mu_{l}\right) \delta_{h}^{\prime} \zeta_{3} \\
& -(1-\alpha)\left(1-\mu_{h}-\mu_{b}-\mu_{l}\right) \delta_{h}^{\prime} \zeta_{5}+\zeta_{4} \zeta_{3}+\zeta_{5} \zeta_{7}
\end{aligned}
$$




$$
\begin{gathered}
\chi_{5}=(1-\alpha)\left(1-\mu_{h}-\mu_{b}-\mu_{l}\right) \delta_{h}^{\prime} \zeta_{2} \\
\chi_{6}=(1-\alpha)\left(1-\mu_{c}\right) \frac{1}{X} \\
\left(1-\mu_{c}\right) \alpha \frac{Y}{X}=X_{w c}^{L}\left(\left(n_{c}^{L}\right)^{1+\xi}+\left(n_{h}^{L}\right)^{1+\xi}\right)^{\frac{\eta-\xi}{1+\xi}}\left(n_{c}^{L}\right)^{1+\xi} \\
\left(1-\mu_{h}-\mu_{b}-\mu_{l}\right) \alpha q I=X_{w h}^{L}\left(\left(n_{c}^{L}\right)^{1+\xi}+\left(n_{h}^{L}\right)^{1+\xi}\right)^{\frac{\eta-\xi}{1+\xi}}\left(n_{h}^{L}\right)^{1+\xi} \\
\frac{n_{h}^{L}}{n_{c}^{L}}=\left(\frac{\left(1-\mu_{h}-\mu_{b}-\mu_{l}\right) q I X_{w c}^{L}}{\left(1-\mu_{c}\right) Y X_{w h}^{L}}\right)^{\frac{1}{1+\xi}} \\
\left(1-\mu_{c}\right) \alpha \frac{Y}{X}=X_{w c}^{L}\left(\frac{\left(1-\mu_{h}-\mu_{b}-\mu_{l}\right) q I X_{w c}^{L}}{\left(1-\mu_{c}\right) Y X_{w h}^{L}}\right)^{\frac{\eta-\xi}{1+\xi}}\left(n_{c}^{L}\right)^{1+\eta} \\
n_{c}^{L}=\left(\frac{\left(1-\mu_{c}\right) \alpha \frac{Y}{X_{w}^{L} X_{c}^{L}}}{\left(1+\frac{\left(1-\mu_{h}-\mu_{b}-\mu_{l}\right) q I X}{\left(1-\mu_{c}\right) Y}\right)^{\frac{\eta-\xi}{1+\xi}}}\right)^{\frac{1}{1+\eta}}
\end{gathered}
$$

Similar formulas apply to $n_{h}^{L}, n_{c}^{B}$, and $n_{h}^{B}$. Once we calculate the hours worked, we can compute $Y, c^{L}, c^{B}, k_{c}, k_{h}, h^{L}, h^{B}, h^{r}, q, q^{r}, b$, and $I$. 


\section{B.6 Data Sources over 1977Q1-2008Q4}

1. Civilian Noninstitutional Population, BLS, CNP160V

2. Real Personal Consumption Expenditure (divided by CNP160V), BEA, NIPA table 1.1.6

3. Real Private Nonresidential Fixed Investment (divided by CNP160V), BEA, NIPA table 1.1.6

4. Real Private Residential Fixed Investment (divided by CNP160V), BEA, NIPA, table 1.1.6

5. Inflation: Log differences in the implicit price deflator for the nonfarm business sector, Demeaned, BLS

6. Nominal interest rate: 3-month Treasury Bill Rate, Demeaned, FRED

7. Real Shiller House Price Index: Shiller House Price Index (FERD) deflated with the implicit price deflator for the nonfarm business sector.

8. Wage Inflation in Consumption-good Sector: Quarterly changes in Average Hourly Earnings of Production/Nonsupervisory Workers on Private Nonfarm Payrolls, Demeaned, BLS

9. Wage Inflation in Housing Sector: Quarterly changes in Average Hourly Earnings of Production/Nonsupervisory Workers in the Construction Industry, Demeaned, BLS 


\section{B.7 Uncertainty-Return Relationship}

The optimal conditions with respect to $h_{t}^{B}$ and $h_{t}^{r}$ can be simplified as:

$$
1=E_{t}\left[\Lambda_{t+1}\left(\frac{(1-\delta) q_{t+1}+\frac{\kappa}{1-\kappa}\left(\frac{h_{t}^{B}}{h_{t}^{r}}\right)^{\xi_{h}-2} q_{t+1}^{r}+m q_{t+1} D_{t+1}}{q_{t}}\right)\right]
$$

where $\Lambda_{t+1}=\beta^{B} \frac{u_{c^{B}, t+1}^{B}}{u_{c^{B}, t}^{B}}$ indicates the present value of marginal utility of future consumption, $D_{t+1}=\frac{\pi_{t+1} u_{b, t}^{B}}{R_{t} \beta^{B} u_{c^{B}, t+1}^{B}}$ is the present value of borrowing costs relative to the present value of future consumption, $q_{t+1}$ is house price, and $q_{t+1}^{r}$ represents rental rate of housing.

The intertemporal marginal rate of substitution is assumed to follow

$$
\Lambda_{t+1}=\exp \left(-r_{t}^{f}-\frac{1}{2} \sigma_{\Lambda, t}^{2}+\epsilon_{\Lambda, t+1}\right)
$$

where $\epsilon_{\Lambda, t+1} \mid I_{t} \sim N\left(0, \sigma_{\Lambda, t}^{2}\right)$. The growth rate of house price and rent is assumed to follow the $\mathrm{AR}(1)$ process.

$$
x_{t+1}^{j}=\alpha_{0}^{j}+\alpha_{1}^{j} x_{t}^{j}+\epsilon_{j, t+1}
$$

where $\epsilon_{j, t+1} \mid I_{t} \sim N\left(0, \sigma_{j, t}^{2}\right)$ and $j$ indexes housing price $(q)$, rent $\left(q^{r}\right)$, and present value of marginal utility of future consumption $(D)$. For example, $x_{t+1}^{q}=\ln \frac{q_{t+1}}{q_{t}}$, $x_{t+1}^{q^{r}}=\ln \frac{q_{t+1}^{r}}{q_{t}^{r}}$, and $x_{t+1}^{D}=\ln \frac{\pi_{t+1} u_{b, t}^{B}}{R_{t} \beta^{B} u_{c}^{B}, t+1}$.

The equation (B.106) can be re-expressed as:

$$
1=E_{t}\left[\Lambda_{t+1} R_{t+1}\right]
$$

where $R_{t+1}=\frac{(1-\delta) q_{t+1}+\frac{\kappa}{1-\kappa}\left(\frac{h_{t}^{B}}{h_{t}^{r}}\right)^{\xi_{h^{-2}}} q_{t+1}^{r}+m q_{t+1} D_{t+1}}{q_{t}}$. By applying Campbell and Shiller's 
(1988) approximation, we derive the following equation.

$$
\begin{aligned}
r_{t+1} & =\ln R_{t+1} \\
& =A+B x_{t+1}^{q}+C x_{t}^{H} \\
& +D x_{t+1}^{q^{r}}+E x_{t+1}^{Q}+F x_{t+1}^{D}
\end{aligned}
$$

where $A=(1-\delta)+\frac{\kappa}{1-\kappa}\left(\frac{h^{B}}{h^{r}}\right)^{\xi_{h}-2}\left(\frac{q^{r}}{q}\right)+m\left(\frac{\beta^{L}}{\beta^{B}}-1\right), B=\left((1-\delta)+m\left(\frac{\beta^{L}}{\beta^{B}}-1\right)\right)$, $C=\frac{\kappa}{1-\kappa}\left(\xi_{h}-2\right)\left(\frac{h^{B}}{h^{r}}\right)^{\xi_{h}-2}\left(\frac{q^{r}}{q}\right), D=\frac{\kappa}{1-\kappa}\left(\frac{h^{B}}{h^{r}}\right)^{\xi_{h}-2}\left(\frac{q^{r}}{q}\right), E=\frac{\kappa}{1-\kappa}\left(\frac{h^{B}}{h^{r}}\right)^{\xi_{h}-2}\left(\frac{q^{r}}{q}\right)$, $F=m\left(\frac{\beta^{L}}{\beta^{B}}-1\right), x_{t}^{H}=\ln \left(\frac{h_{t}^{B}}{h_{t}^{r}}\right)$ and $x_{t}^{Q}=\ln \left(\frac{q_{t}^{r}}{q_{t}}\right)$. Assume that a solution to the $\log$ of rent to house price ratio takes the following form:

$$
x_{t+1}^{Q}=c_{0}+c_{1} x_{t}^{q}+c_{2} x_{t}^{H}+c_{3} x_{t}^{q^{r}}+c_{4} x_{t}^{D}+c_{5} \sigma_{q t}^{2}+c_{6} \sigma_{q^{r} t}^{2}+c_{7} \sigma_{D t}^{2}
$$

Substituting equations (B.110) and (B.111),

$$
1=E_{t} \exp [A(\cdot)]
$$

where

$$
\begin{aligned}
A(\cdot) & =\left(-r^{f}+A+B \alpha_{0}^{q}+D \alpha_{0}^{q^{r}}+E c_{0}+F \alpha_{0}^{D}\right)-\frac{1}{2} \sigma_{\Lambda t}^{2}+\varepsilon_{\Lambda t+1} \\
& +\left(B \alpha_{1}^{q}+E c_{1}\right) x_{t}^{q}+\left(C+E c_{2}\right) x_{t}^{H}+\left(D \alpha_{1}^{q^{r}}+E c_{3}\right) x_{t}^{q^{r}}+\left(F \alpha_{1}^{D}+E c_{4}\right) x_{t}^{D} \\
& +E c_{5} \sigma_{q t}^{2}+E c_{6} \sigma_{q^{r} t}^{2}+E c_{7} \sigma_{D t}^{2}+B \varepsilon_{q t+1}+D \varepsilon_{q^{r} t+1}+F \varepsilon_{D t+1}
\end{aligned}
$$


The first and second moments are as follows:

$$
\begin{aligned}
E_{t} A(\cdot) & =\left(-r^{f}+A+B \alpha_{0}^{q}+D \alpha_{0}^{q^{r}}+E c_{0}+F \alpha_{0}^{D}\right)-\frac{1}{2} \sigma_{\Lambda t}^{2} \\
& +\left(B \alpha_{1}^{q}+E c_{1}\right) x_{t}^{q}+\left(C+E c_{2}\right) x_{t}^{H}+\left(D \alpha_{1}^{q^{r}}+E c_{3}\right) x_{t}^{q^{r}}+\left(F \alpha_{1}^{D}+E c_{4}\right) x_{t}^{D} \\
& +E c_{5} \sigma_{q t}^{2}+E c_{6} \sigma_{q^{r} t}^{2}+E c_{7} \sigma_{D t}^{2}
\end{aligned}
$$

$$
\begin{aligned}
\operatorname{Var}_{t} A(\cdot) & =\sigma_{\Lambda t}^{2}+B^{2} \sigma_{q t}^{2}+D^{2} \sigma_{q^{r} t}^{2}+F^{2} \sigma_{D t}^{2}+2 B \rho_{q \Lambda} \sigma_{q t}^{2} \\
& +2 B D \rho_{q q^{r}} \sigma_{q t}^{2}+2 B F \rho_{q D} \sigma_{q t}^{2}
\end{aligned}
$$

Since $A(\cdot)$ follows a normal distribution, the equation (B.112) leads to

$$
E_{t} A(\cdot)+\frac{1}{2} \operatorname{Var}_{t} A(\cdot)=0
$$

By substituting the equations (B.114) and (B.115), this leads to

$$
\begin{aligned}
0= & \left(-r^{f}+A+B \alpha_{0}^{q}+D \alpha_{0}^{q^{r}}+E c_{0}+F \alpha_{0}^{D}\right) \\
& +\left(B \alpha_{1}^{q}+E c_{1}\right) x_{t}^{q}+\left(C+E c_{2}\right) x_{t}^{H}+\left(D \alpha_{1}^{q^{r}}+E c_{3}\right) x_{t}^{q^{r}}+\left(F \alpha_{1}^{D}+E c_{4}\right) x_{t}^{D} \\
& +\left(\frac{1}{2} B^{2}+B \rho_{q \Lambda}+B D \rho_{q q^{r}}+B F \rho_{q D}+E c_{5}\right) \sigma_{q t}^{2} \\
& +\left(\frac{1}{2} D^{2}+E c_{6}\right) \sigma_{q^{r} t}^{2}+\left(\frac{1}{2} F^{2}+E c_{7}\right) \sigma_{D t}^{2}
\end{aligned}
$$

This equation holds when the terms in the eight brackets must be equal to zero. Based on this result, we derive the following rent to house price ratio:

$$
x_{t+1}^{Q}=q_{t}^{r}-q_{t}=c_{0}+c_{1} x_{t}^{q}+c_{2} x_{t}^{H}+c_{3} x_{t}^{q^{r}}+c_{4} x_{t}^{D}+c_{5} \sigma_{q t}^{2}+c_{6} \sigma_{q^{r} t}^{2}+c_{7} \sigma_{D t}^{2}
$$


where

$$
\begin{aligned}
& c_{0}=-\frac{-r^{f}+A+B \alpha_{0}^{q}+D \alpha_{0}^{q^{r}}+F \alpha_{0}^{D}}{E} \\
& c_{1}=-\frac{B \alpha_{1}^{q}}{E} \\
& c_{2}=-\frac{C}{E} \\
& c_{3}=-\frac{D \alpha_{1}^{q^{r}}}{E} \\
& c_{4}=-\frac{F \alpha_{1}^{D}}{E} \\
& c_{5}=-\frac{\frac{1}{2} B^{2}+B \rho_{q \Lambda}+B D \rho_{q q^{r}}+B F \rho_{q D}}{E} \\
& c_{6}=-\frac{\frac{1}{2} D^{2}}{E} \\
& c_{7}=-\frac{\frac{1}{2} F^{2}}{E}
\end{aligned}
$$

By substituting the equation (B.118) into (B.110), we get

$$
\begin{aligned}
r_{t+1} & =r^{f}+B \varepsilon_{q t+1}+D \varepsilon_{q^{r} t+1}+F \varepsilon_{D t+1} \\
& -\left(\frac{1}{2} B^{2}+B \rho_{q \Lambda}+B D \rho_{q q^{r}}+B F \rho_{q D}\right) \sigma_{q t}^{2}-\frac{1}{2} D^{2} \sigma_{q^{r} t}^{2}-\frac{1}{2} F^{2} \sigma_{D t}^{2}
\end{aligned}
$$

We obtain the following first and second moments of $r_{t+1}$

$$
\begin{aligned}
& E_{t} r_{t+1}=r^{f}-\left(\frac{1}{2} B^{2}+B \rho_{q \Lambda}+B D \rho_{q q^{r}}+B F \rho_{q D}\right) \sigma_{q t}^{2}-\frac{1}{2} D^{2} \sigma_{q^{r} t}^{2}-\frac{1}{2} F^{2} \sigma_{D t}^{2} \\
& \operatorname{Var}_{t} r_{t+1}=B^{2} \sigma_{q t}^{2}+D^{2} \sigma_{q^{r} t}^{2}+F^{2} \sigma_{D t}^{2}+2 B D \rho_{q q^{r}} \sigma_{q t}^{2}+2 B F \rho_{q D} \sigma_{q t}^{2}
\end{aligned}
$$

This leads to

$$
\frac{\partial E_{t} r_{t+1}}{\partial \operatorname{Var}_{t} r_{t+1}}=-\frac{\frac{1}{2} B+\rho_{q \Lambda}+D \rho_{q q^{r}}+F \rho_{q D}}{B+2 D \rho_{q q^{r}}+2 F \rho_{q D}}
$$

The above derivation applies to the uncertainty-return relationship for lenders and the real-options channel (equation (2.43) and equation (2.46)). 


\section{B.8 Additional Analysis}

\section{Additional VAR analysis}

\section{(1) Non-regime dependent VAR model}

As an additional analysis, we consider a quarterly, two-lags VAR model with a constant term and a linear time trend. The constant VAR model with symmetric information flow allows us to investigate the average impulse responses over all the sample periods. The model is represented as follows:

$$
Y_{t}=c+\tau_{t}+\sum_{i=1}^{2} \phi_{i} Y_{t-i}+\Sigma^{1 / 2} \varepsilon_{t}, \quad \varepsilon_{t} \sim N(0,1)
$$

where $\Sigma=A^{-1} A^{-1^{\prime}}, A$ denotes the contemporaneous relationship between reducedform shocks, $c$ is a constant term, $\tau_{t}$ is a linear time trend, and $Y_{t}$ is a vector of endogenous variables. We take a log transformation for all variables without detrending, except for CPI inflation, Treasury bill rate, and mortgage rate. We estimate the model based on a Minnesota prior and compute credible sets from the posterior.

Figure 11 shows that the house price uncertainty shock dampens economic activity, which mostly resembles the impulse responses of the recessionary regimes in the benchmark VAR analysis. It initially reduces consumption and residential investment by $0.2 \%$ and $0.9 \%$, respectively. Both of the responses are quite persistent, with their peak responses occurring after about 20 quarters. The peak decline in residential investment is around four times as large as the peak decline in consumption. Inflation falls, though it is not statistically significant. The monetary policy rate drops and remains below the trend for about three years. The decrease in the monetary policy rate leads to a fall in mortgage rates but to a smaller extent, reducing the mortgage spread. Real house prices and mortgage debt decrease by $1.0 \%$ and $2.5 \%$ at the peak, respectively. These results imply that the financial and debt risk effect would be 
stronger than the hedging effect over the whole sample. Finally, the rent-to-price ratio starts increasing at a 10 quarter horizon and reverts back to the original level in the long run.

\section{(2) Max Share Identification}

For robustness analysis, we identify a house price uncertainty shock that maximizes the forecast error variance (FEV) of house price uncertainty at a finite forecast horizon (Max Share). ${ }^{1}$ The model is based on the constant VAR model (equation (B.122)). The forecast horizon is set to 40 quarters. As an alternative to long-run restrictions, the Max Share identification scheme proposed by Uhlig (2005) might not only capture short- and medium-run fluctuations of house price uncertainty but also provide better estimation precision over the long-run restrictions (Francis et al., 2014). This section limits our attention to extracting the first largest shock that explains most of the movements in house price uncertainty.

Figure 12 shows the impulse responses to the Max Share house price uncertainty shock. All the responses generated by the Max Share algorithm are similar to the responses with short-run restriction in the constant VAR analysis, except for the response of consumption and housing prices. Consumption and housing prices display positive responses to the shock in the short run. Housing prices gradually increase up to 7 quarters but revert to the trend level at 17 quarters. In the long-run, housing prices decrease to a lower level. This implies that the Max Share identification is more likely to capture the hedging effect of house price uncertainty than the short-

\footnotetext{
${ }^{1}$ The $k$-step ahead forecast error of the $i$ th variable can be expressed as:

$$
y_{i, t+k}-E_{t} y_{i, t+k}=e_{i}^{\prime}\left[\sum_{l=0}^{k-1} C_{l} \tilde{A} Q u_{t+k-l}\right],
$$

such that $\varepsilon_{t}=A u_{t}$ and $\tilde{A Q}=A$, where $Q$ is an orthonormal matrix. The Max Share identifies the shock by solving

$$
\max e_{i}^{\prime}\left[\sum_{k=\bar{k}}^{\bar{k}} \sum_{l=0}^{k-1} C_{l} \tilde{A} q q^{\prime} \tilde{A}^{\prime} C_{l}^{\prime}\right] e_{i},
$$

where $q$ is the column of $Q$ defining the most important shock.
} 
run restriction does. The rise in housing prices leads to an increase in consumption through the wealth effects and collateral constraints channel. 

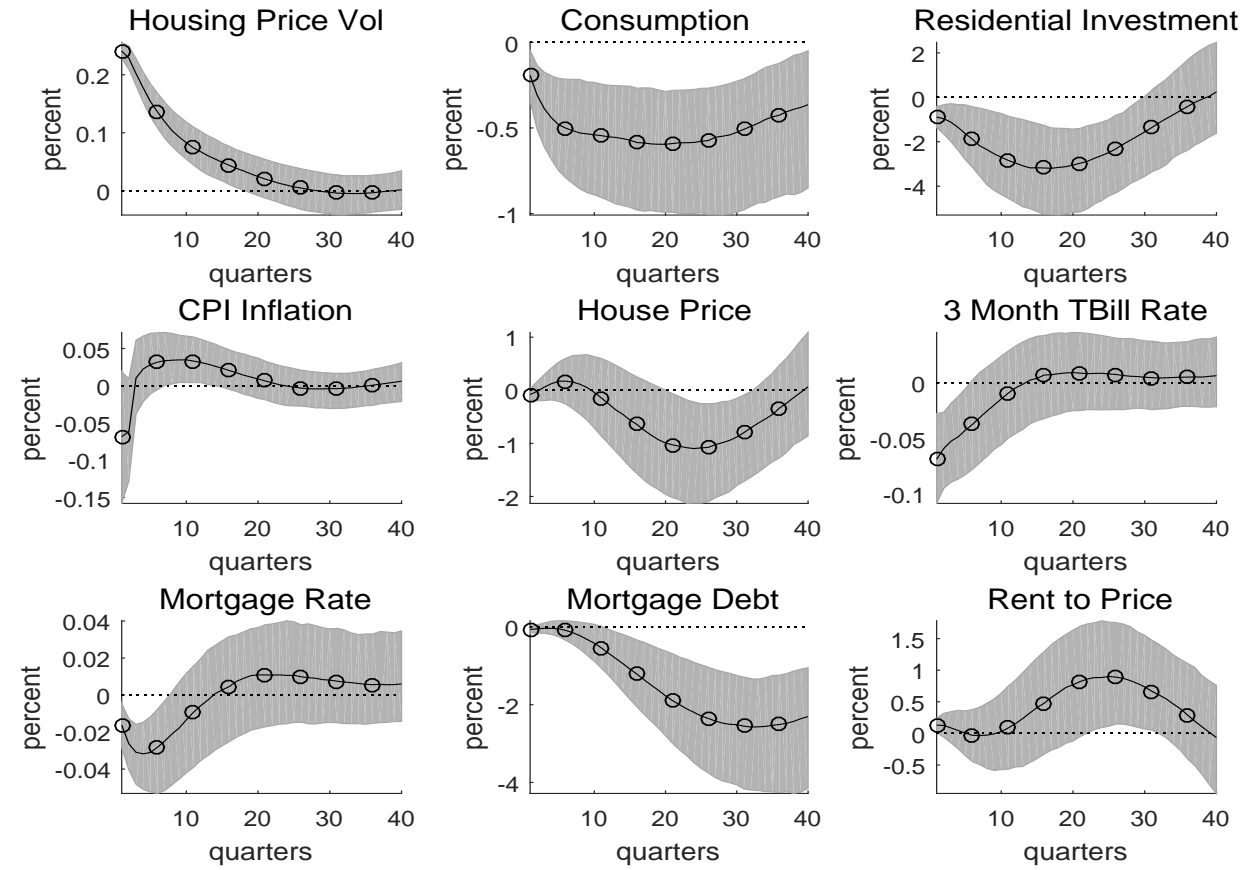

Figure B.1: Impulse Responses to House Price Uncertainty Shock: Constant
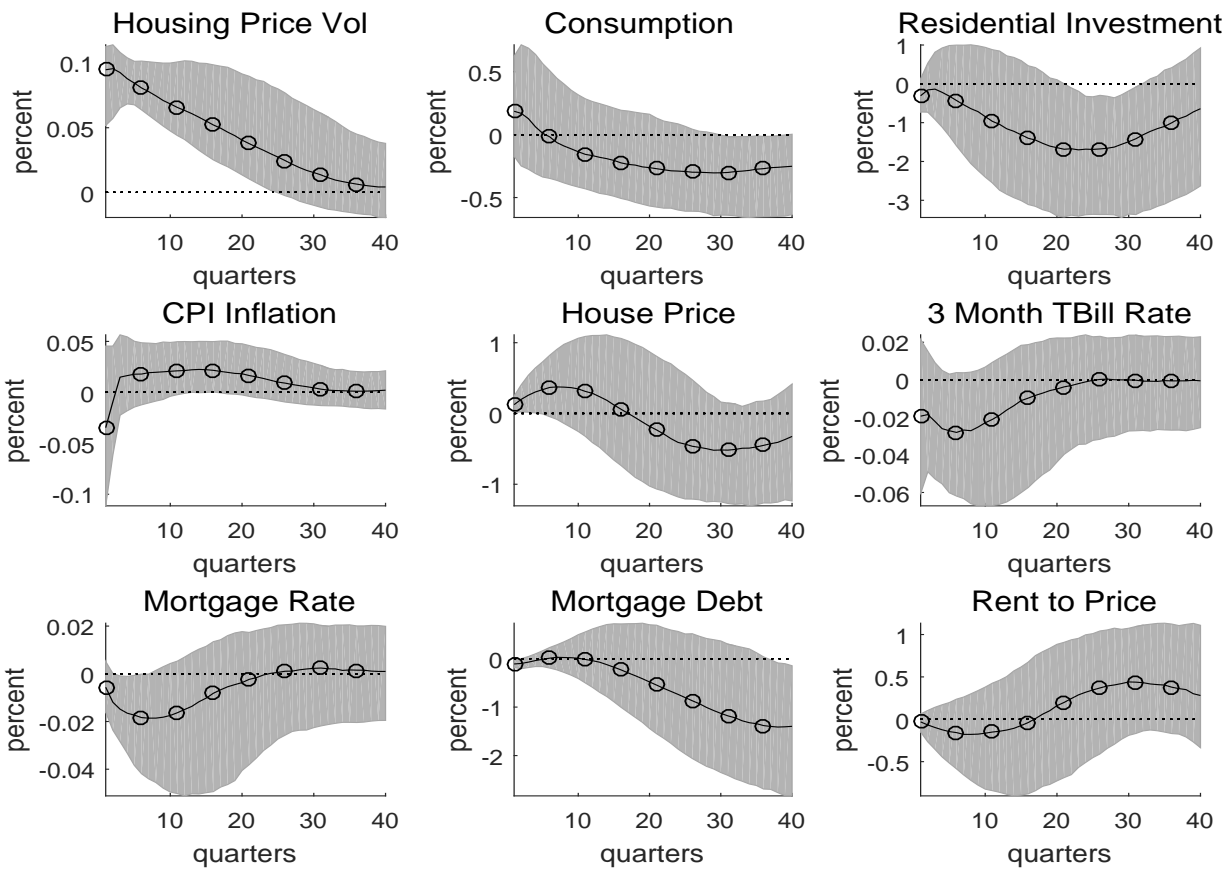

Figure B.2: Impulse Responses to House Price Uncertainty Shock: Max Share 
Table B.1 State-level evidence for financial risk and hedging effect

\begin{tabular}{l|c|c|c|c}
\hline \multicolumn{5}{c}{ Whole Sample (2001-2017) } \\
\hline & Coef. & Std. Err. & t-value & p-value \\
\hline$\beta_{q \Lambda}$ & -0.0003 & 0.004 & -0.07 & 0.941 \\
$\beta_{q q^{r}}$ & 0.028 & 0.020 & 1.43 & 0.153 \\
\hline Subsample $1(2001-2007)$ \\
\hline$\beta_{q \Lambda}$ & -0.0008 & 0.007 & -0.1 & 0.919 \\
$\beta_{q q^{r}}$ & 4.272 & 0.938 & 4.56 & 0 \\
\hline Subsample 2 (2007-2017) \\
\hline$\beta_{q \Lambda}$ & -0.0003 & 0.005 & -0.07 & 0.948 \\
$\beta_{q q^{r}}$ & 0.027 & 0.019 & 1.41 & 0.16 \\
\hline
\end{tabular}



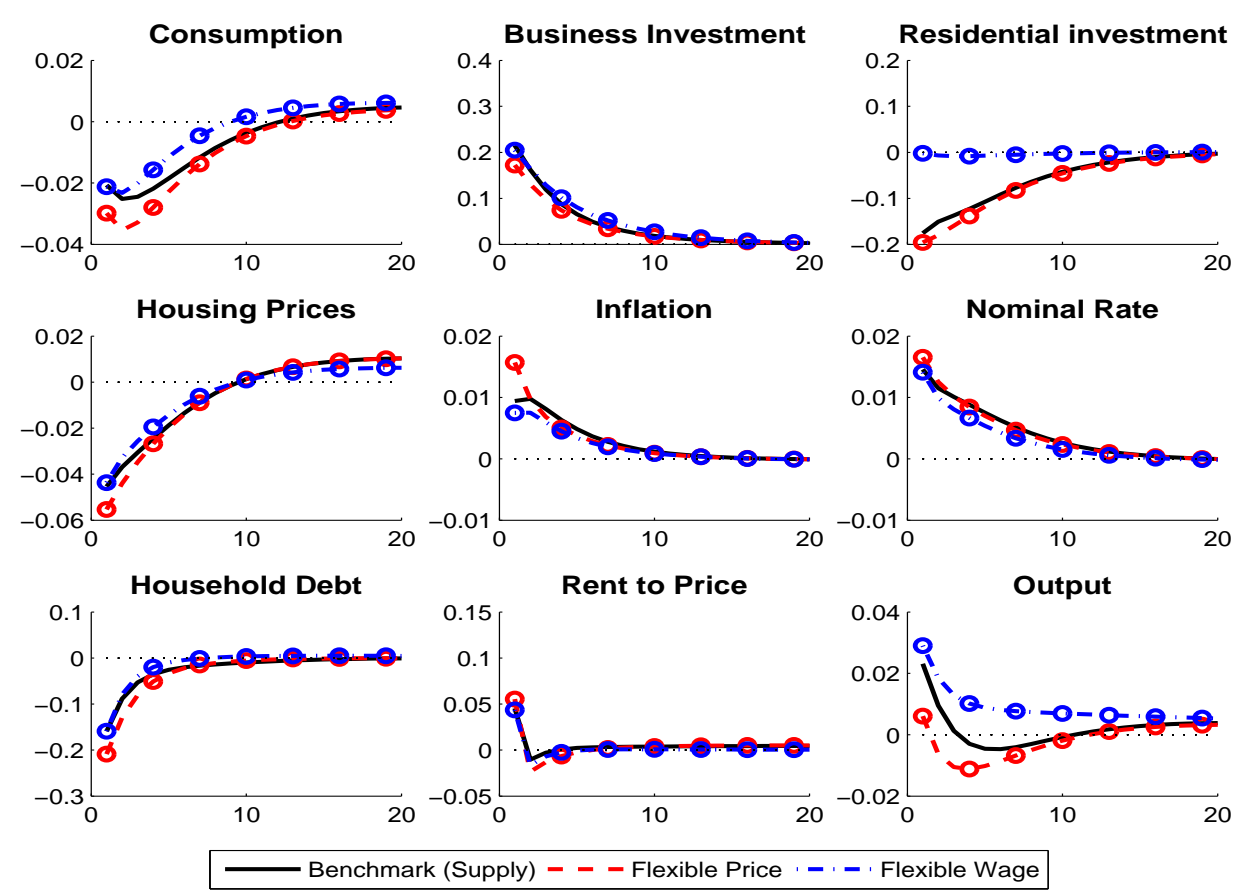

Figure B.3: Responses to Supply-side Uncertainty Shock: Role of price and wage stickiness
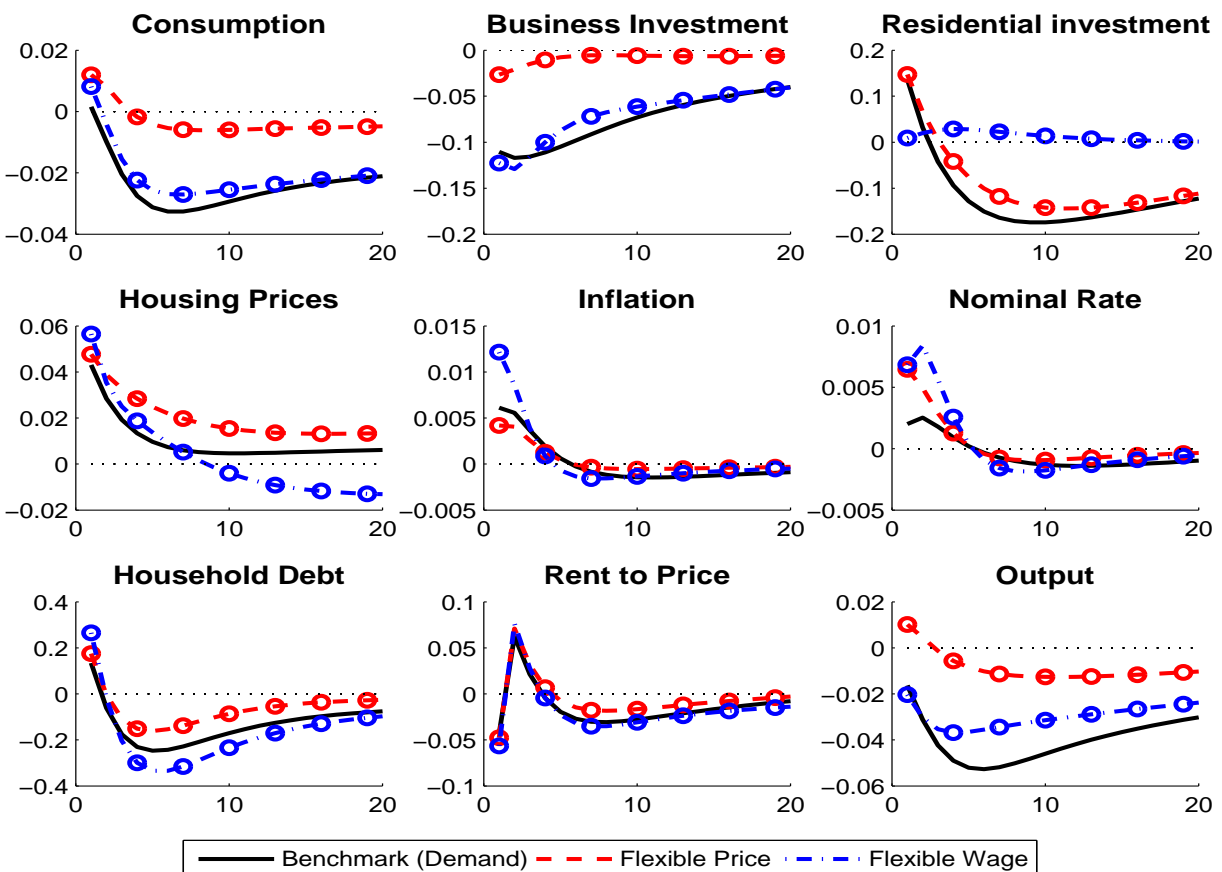

Figure B.4: Responses to Demand-side Uncertainty Shock: Role of price and wage stickiness 

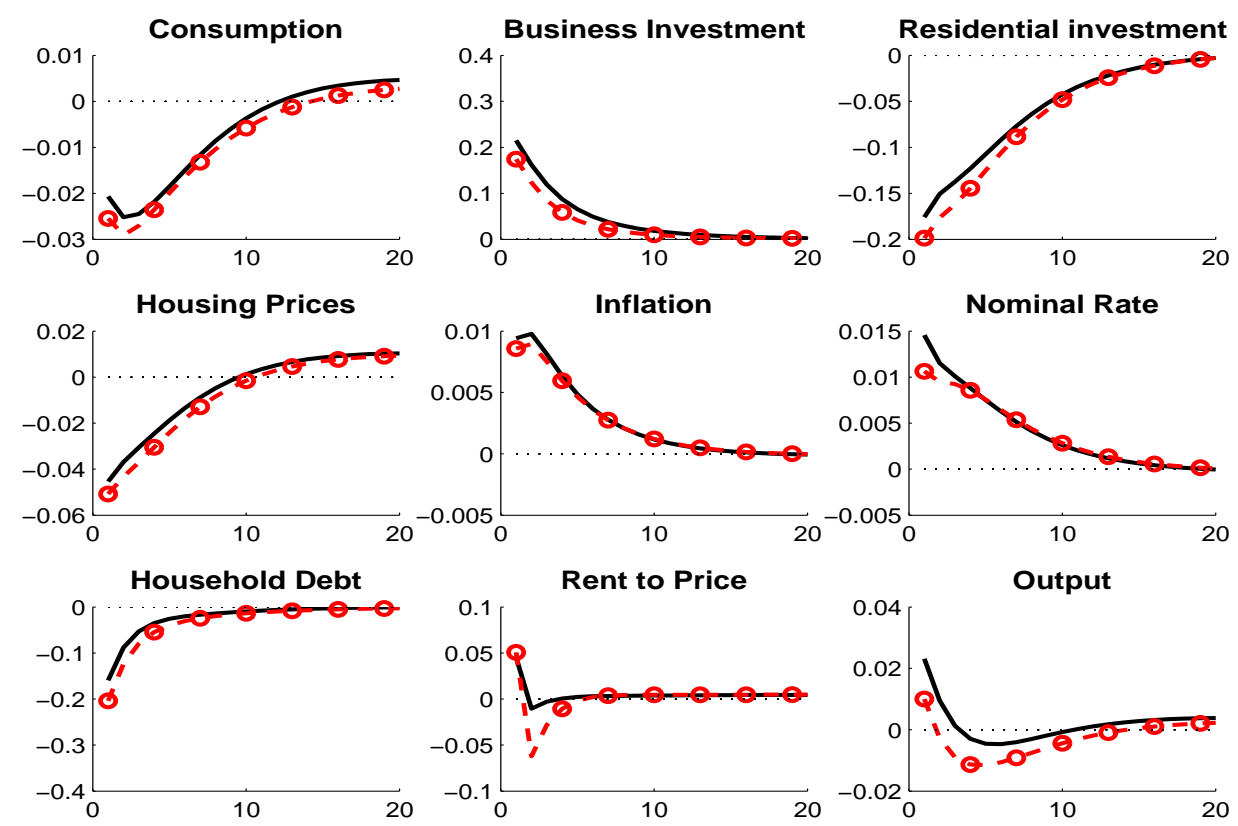

Figure B.5: Responses to Supply-side Uncertainty Shock: Role of collateral constraints
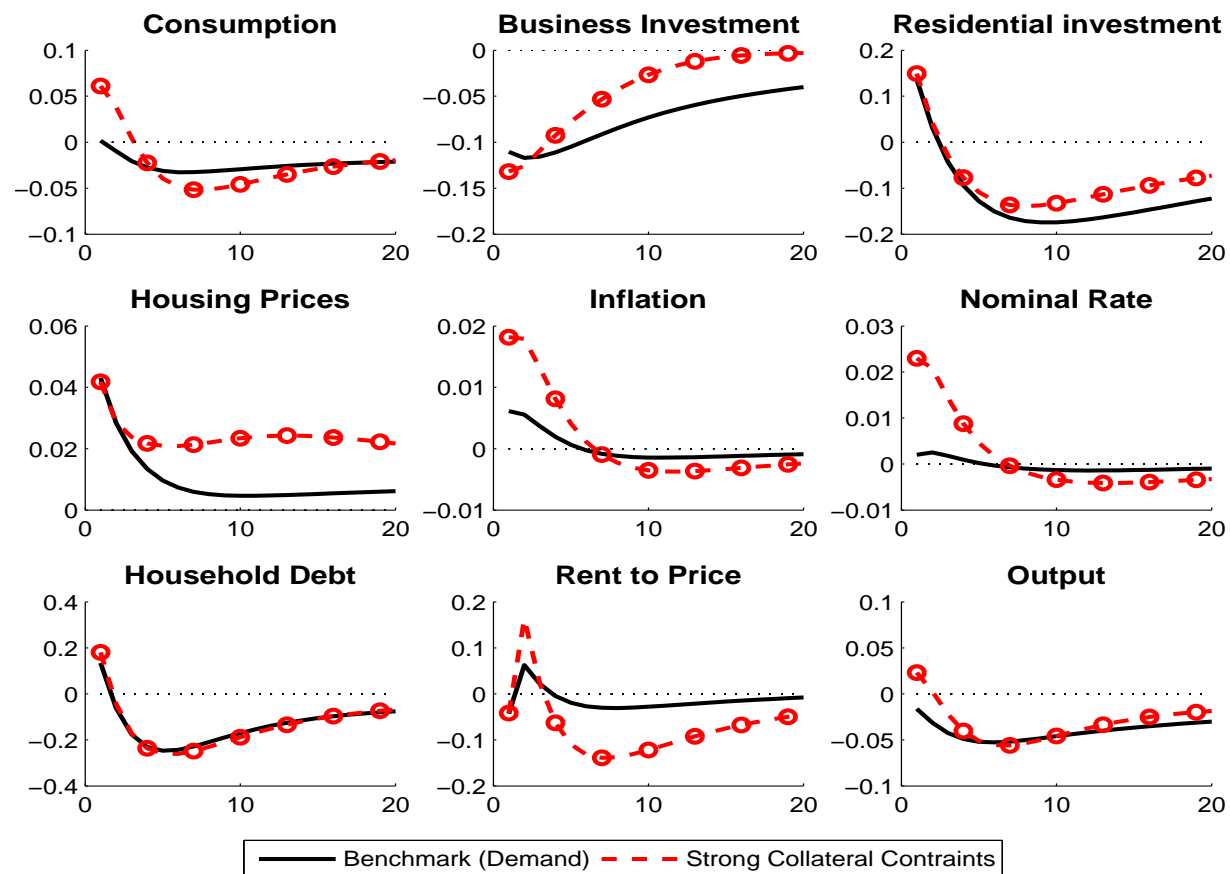

Figure B.6: Responses to Demand-side Uncertainty Shock: Role of collateral constraints 

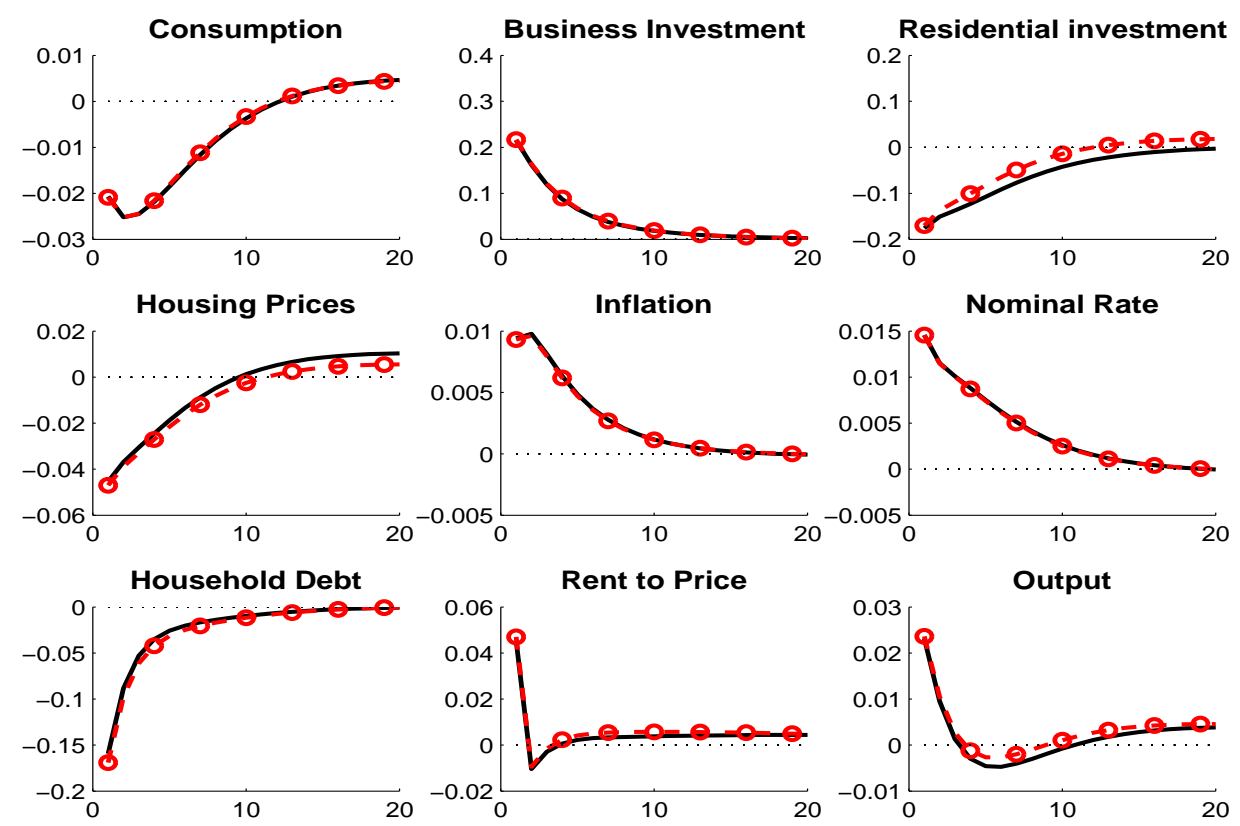

Figure B.7: Responses to Supply-side Uncertainty Shock: Role of perfect factor mobility
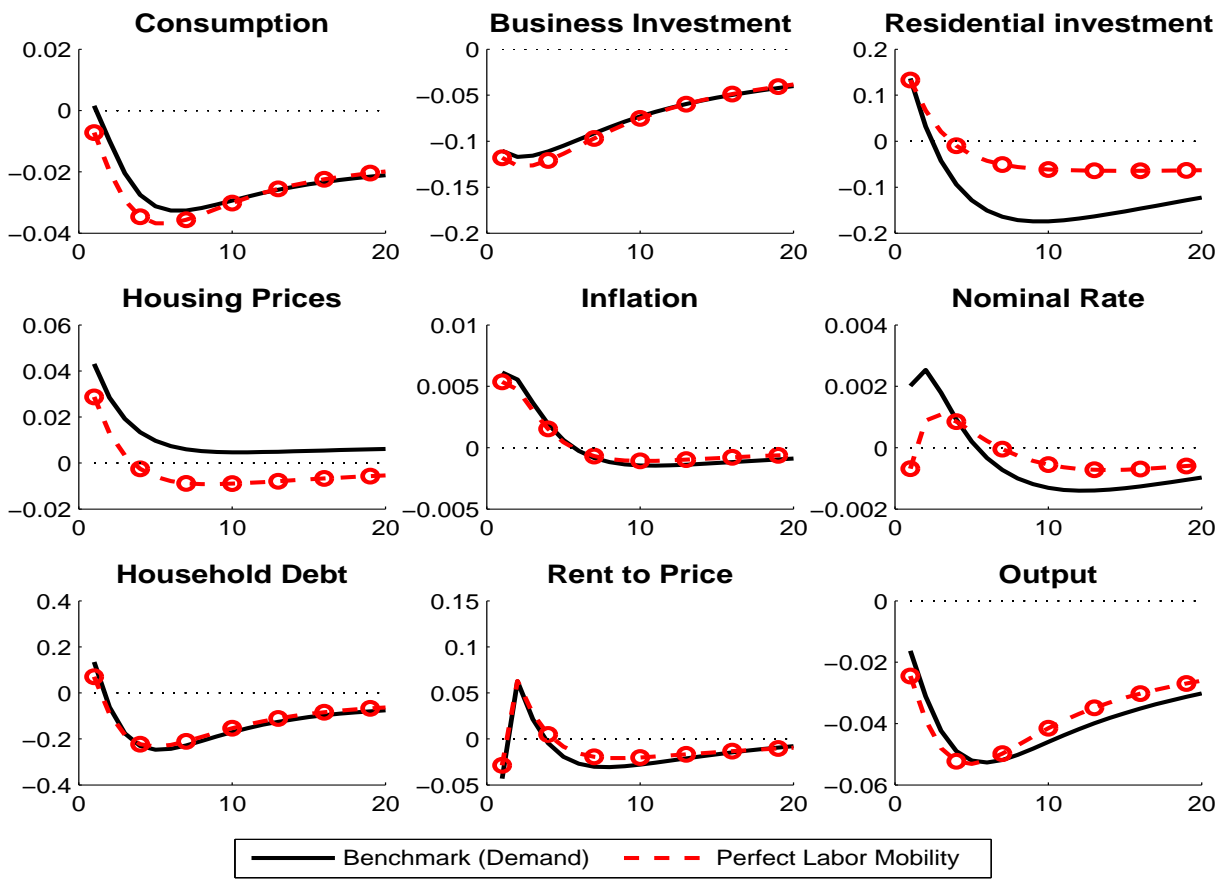

Figure B.8: Responses to Demand-side Uncertainty Shock: Role of perfect factor mobility 


\section{Bibliography}

Abel, Andrew B (1983) "Optimal investment under uncertainty," American Economic Review, Vol. 73, pp. 228-233.

Achieser, Naum I (2013) Theory of approximation: Courier Corporation.

Adjemian, Stéphane, Houtan Bastani, Michel Juillard, Ferhat Mihoubi, George Perendia, Marco Ratto, and Sébastien Villemot (2011) "Dynare: Reference manual, version 4."

Aguiar, Mark and Gita Gopinath (2007) "Emerging market business cycles: The cycle is the trend," Journal of political Economy, Vol. 115, pp. 69-102.

Ali-Löytty, Simo (2008) On the convergence of the gaussian mixture filter: Tampere University of Technology.

Alspach, Daniel L and Harold W Sorenson (1971) "Recursive Bayesian estimation using Gaussian sums," Automatica, Vol. 7, pp. 465-479, DOI: http://dx.doi. org/10.1016/0005-1098(71)90097-5.

_ (1972) "Nonlinear Bayesian estimation using Gaussian sum approximations," Automatic Control, IEEE Transactions on, Vol. 17, pp. 439-448, DOI: http://dx . doi.org/10.1109/TAC.1972.1100034.

Alvarez-Parra, Fernando, Luis Brandao-Marques, and Manuel Toledo (2013) "Durable goods, financial frictions, and business cycles in emerging economies," Journal of Monetary Economics, Vol. 60, pp. 720-736.

Amisano, Gianni and Oreste Tristani (2010) "Euro area inflation persistence in an estimated nonlinear DSGE model," Journal of Economic Dynamics and Control, Vol. 34, pp. 1837-1858, DOI: http://dx.doi.org/10.1016/j.jedc.2010.05.001.

An, Sungbae and Frank Schorfheide (2007) "Bayesian analysis of DSGE models," Econometric Reviews, Vol. 26, pp. 113-172, DOI: http://dx.doi.org/10.1080/ 07474930701220071. 
Andreasen, Martin M (2011) "Non-linear DSGE models and the optimized central difference particle filter," Journal of Economic Dynamics and Control, Vol. 35, pp. 1671-1695, DOI: http://dx.doi.org/10.1016/j.jedc.2011.04.007.

(2012) "On the effects of rare disasters and uncertainty shocks for risk premia in non-linear DSGE models," Review of Economic Dynamics, Vol. 15, pp. 295-316, DOI: http://dx.doi.org/10.1016/j.red.2011.08.001.

(2013) "Non-Linear DSGE Models And The Central Difference Kalman Filter," Journal of Applied Econometrics, Vol. 28, pp. 929-955, DOI: http: //dx.doi.org/10.1002/jae.2282.

Andreasen, Martin M, Jesús Fernández-Villaverde, and Juan F Rubio-Ramírez (2017) "The pruned state-space system for non-linear DSGE models: Theory and empirical applications," Review of Economic Studies, Vol. 85, pp. 1-49.

Andrieu, Christophe, Arnaud Doucet, and Roman Holenstein (2010) "Particle markov chain monte carlo methods," Journal of the Royal Statistical Society: Series B (Statistical Methodology), Vol. 72, pp. 269-342, DOI: http://dx.doi.org/10.1111/j . 1467-9868.2009.00736.x.

Andrieu, Christophe and Gareth O Roberts (2009) "The pseudo-marginal approach for efficient Monte Carlo computations," The Annals of Statistics, Vol. 37, pp. 697-725, URL: http://www.jstor.org.proxy.mul.missouri.edu/stable/ 30243645.

Arasaratnam, Ienkaran and Simon Haykin (2009) "Cubature Kalman filters," $A u$ tomatic Control, IEEE Transactions on, Vol. 54, pp. 1254-1269, DOI: http: //dx.doi.org/10.1109/TAC.2009.2019800.

Bańbura, Marta, Domenico Giannone, and Lucrezia Reichlin (2010) "Large Bayesian vector auto regressions," Journal of Applied Econometrics, Vol. 25, pp. 71-92.

Banks, James, Richard Blundell, Zoe Oldfield, and James P Smith (2004) "Housing wealth over the life-cycle in the presence of housing price volatility," Institute for Fiscal Studies, mimeo (September).

Basu, Susanto and Brent Bundick (2017) "Uncertainty shocks in a model of effective demand," Econometrica, Vol. 85, pp. 937-958, DOI: http://dx.doi.org/10. 3982/ECTA13960.

Berkovec, James and Don Fullerton (1992) "A general equilibrium model of housing, taxes, and portfolio choice," Journal of Political Economy, Vol. 100, pp. 390-429.

Bernanke, Ben S (1983) "The determinants of investment: Another look," American Economic Review, Vol. 73, pp. 71-75. 
Bernanke, Ben S, Mark Gertler, and Simon Gilchrist (1999) "The financial accelerator in a quantitative business cycle framework," Handbook of Macroeconomics, Vol. 1, pp. 1341-1393.

Binning, Andrew and Junior Maih (2015) "Sigma point filters for dynamic nonlinear regime switching models, Working Paper, Norges Bank," URL: https://ideas. repec.org/p/bno/worpap/2015_10.html.

Bloom, Nicholas (2009) "The impact of uncertainty shocks," Econometrica, Vol. 77, pp. $623-685$.

(2014) "Fluctuations in uncertainty," Journal of Economic Perspectives, Vol. 28 , pp. $153-175$.

Bollerslev, Tim and Jeffrey M Wooldridge (1992) "Quasi-maximum likelihood estimation and inference in dynamic models with time-varying covariances," Econometric Reviews, Vol. 11, pp. 143-172, DOI: http://dx.doi.org/10.1080/ 07474939208800229 .

Bonciani, Dario and Björn van Roye (2016) "Uncertainty shocks, banking frictions and economic activity," Journal of Economic Dynamics and Control, Vol. 73, pp. 200-219, DOI: http://dx.doi.org/10.1016/j.jedc.2016.09.008.

Born, Benjamin and Johannes Pfeifer (2014a) "Policy risk and the business cycle," Journal of Monetary Economics, Vol. 68, pp. 68-85, DOI: http://dx.doi.org/ 10.1016/j. jmoneco.2014.07.012.

(2014b) "Risk matters: The real effects of volatility shocks: Comment," American Economic Review, Vol. 104, pp. 4231-4239.

Boz, Emine, Christian Daude, and C Bora Durdu (2011) "Emerging market business cycles: Learning about the trend," Journal of Monetary Economics, Vol. 58, pp. 616-631.

Brock, William A and Leonard J Mirman (1972) "Optimal economic growth and uncertainty: the discounted case," Journal of Economic Theory, Vol. 4, pp. 479513, DOI: http://dx.doi.org/10.1016/0022-0531(72)90135-4.

Brueckner, Jan K (1997) "Consumption and investment motives and the portfolio choices of homeowners," The Journal of Real Estate Finance and Economics, Vol. 15, pp. 159-180.

Campbell, John Y and Joao F Cocco (2007) "How do house prices affect consumption? Evidence from micro data," Journal of Monetary Economics, Vol. 54, pp. 591-621.

Canova, Fabio and Luca Sala (2009) "Back to square one: Identification issues in DSGE models," Journal of Monetary Economics, Vol. 56, pp. 431-449, DOI: http: //dx.doi.org/10.1016/j.jmoneco.2009.03.014. 
Caplin, Andrew, Sewin Chan, Charles Freeman, and Joseph Tracy (1997) Housing Partnerships: MIT Press, Cambridge, Ma.

Carroll, Christopher D, Miles S Kimball et al. (2006) Precautionary saving and precautionary wealth: Univ.-Bibliothek Frankfurt am Main.

Case, Karl E (1994) "Land prices and house prices in the United States," in Housing Markets in the United States and Japan: University of Chicago Press, pp. 29-48.

Case, Karl E, John M Quigley, and Robert J Shiller (2005) "Comparing wealth effects: the stock market versus the housing market," Advances in macroeconomics, Vol. 5.

Cesa-Bianchi, Ambrogio and Emilio Fernández Corugedo (2018) "Uncertainty, Financial Frictions, and Nominal Rigidities: A Quantitative Investigation," Journal of Money, Credit and Banking, Vol. 50, pp. 603-636.

Chang, Roberto and Andrés Fernández (2013) "On the sources of aggregate fluctuations in emerging economies," International Economic Review, Vol. 54, pp. 12651293.

Chopin, Nicolas (2004) "Central limit theorem for sequential Monte Carlo methods and its application to Bayesian inference," The Annals of Statistics, Vol. 32, pp. 2385-2411, DOI: http://dx.doi.org/10.1214/009053604000000698.

Da, Zhi and Hayong Yun (2010) "Electricity consumption and asset prices, Working Paper."

DeJong, David N, Roman Liesenfeld, Guilherme V Moura, Jean-François Richard, and Hariharan Dharmarajan (2012) "Efficient likelihood evaluation of state-space representations," Review of Economic Studies, Vol. 80, pp. 538-567, DOI: http: //dx.doi.org/10.1093/restud/rds040.

Del Negro, Marco and Christopher Otrok (2007) "99 Luftballons: Monetary policy and the house price boom across US states," Journal of Monetary Economics, Vol. 54, pp. 1962-1985.

Den Haan, Wouter J and Joris De Wind (2012) "Nonlinear and stable perturbationbased approximations," Journal of Economic Dynamics and Control, Vol. 36, pp. $1477-1497$.

Fernández-Villaverde, Jesús (2010) "The econometrics of DSGE models," SERIEs, Vol. 1, pp. 3-49, DOI: http://dx.doi.org/10.1007/s13209-009-0014-7.

Fernández-Villaverde, Jesús, Pablo Guerrón-Quintana, Keith Kuester, and Juan Rubio-Ramírez (2015) "Fiscal volatility shocks and economic activity," American Economic Review, Vol. 105, pp. 3352-3384, DOI: http://dx.doi.org/10.1257/ aer. 20121236. 
Fernández-Villaverde, Jesús, Pablo Guerrón-Quintana, Juan F Rubio-Ramírez, and Martin Uribe (2011) "Risk matters: The real effects of volatility shocks," American Economic Review, Vol. 101, pp. 2530-2561, DOI: http://dx.doi.org/10.1257/ aer.101.6.2530.

Fernández-Villaverde, Jesús and Oren Levintal (2016) "Solution methods for models with rare disasters,"Technical report, National Bureau of Economic Research.

Fernández-Villaverde, Jesús and Juan F Rubio-Ramírez (2005) "Estimating dynamic equilibrium economies: linear versus nonlinear likelihood," Journal of Applied Econometrics, Vol. 20, pp. 891-910, DOI: http://dx.doi.org/10.1002/jae.814.

(2007) "Estimating macroeconomic models: A likelihood approach," Review of Economic Studies, Vol. 74, pp. 1059-1087, DOI: http://dx.doi.org/10.1111/ j.1467-937X.2007.00437.x.

Fort, Gersende and Eric Moulines (2000) "V-subgeometric ergodicity for a HastingsMetropolis algorithm," Statistics $\&$ probability letters, Vol. 49, pp. 401-410, DOI: http://dx.doi.org/10.1016/S0167-7152(00)00074-2.

Francis, Neville, Michael T Owyang, Jennifer E Roush, and Riccardo DiCecio (2014) "A flexible finite-horizon alternative to long-run restrictions with an application to technology shocks," Review of Economics and Statistics, Vol. 96, pp. 638-647.

Garcia-Cicco, Javier, Roberto Pancrazi, and Martin Uribe (2010) "Real business cycles in emerging countries?" American Economic Review, Vol. 100, pp. 2510-31.

Gazzani, Andrea Giovanni (2016) "News and noise in the housing market, Working Paper."

Geweke, John (1999) "Using simulation methods for Bayesian econometric models: inference, development, and communication," Econometric Reviews, Vol. 18, pp. 1-73, DOI: http://dx.doi.org/10.1080/07474939908800428.

(2005) Contemporary Bayesian Econometrics and Statistics, Vol. 537: John Wiley \& Sons.

Gómez-González, Patricia, Daniel Rees et al. (2013) Stochastic Terms of Trade Volatility in Small Open Economies: Reserve Bank of Australia.

Green, Richard K and Kerry D Vandell (1999) "Giving households credit: How changes in the US tax code could promote homeownership," Regional Science and Urban Economics, Vol. 29, pp. 419-444.

Hall, Jamie, Michael K Pitt, and Robert Kohn (2014) "Bayesian inference for nonlinear structural time series models," Journal of Econometrics, Vol. 179, pp. 99-111, DOI: http://dx.doi.org/10.1016/j.jeconom.2013.10.016. 
Hall, Peter and Christopher C Heyde (2014) Martingale limit theory and its application: Academic press.

Hamilton, James D (1989) "A new approach to the economic analysis of nonstationary time series and the business cycle," Econometrica, pp. 357-384.

Han, Lu (2010) "The effects of price risk on housing demand: empirical evidence from US markets," Review of Financial Studies, Vol. 23, pp. 3889-3928.

(2013) "Understanding the puzzling risk-return relationship for housing," Review of Financial Studies, Vol. 26, pp. 877-928.

Hansen, Gary D (1985) "Indivisible labor and the business cycle," Journal of Monetary Economics, Vol. 16, pp. 309-327, DOI: http://dx.doi.org/10.1016/ 0304-3932 (85) 90039-X.

Hartman, Richard (1972) "The effects of price and cost uncertainty on investment," Journal of Economic Theory, Vol. 5, pp. 258-266.

(1976) "Factor demand with output price uncertainty," American Economic Review, Vol. 66, pp. 675-681.

Henderson, J Vernon, Yannis M Ioannides et al. (1983) "A model of housing tenure choice," American Economic Review, Vol. 73, pp. 98-113.

Herbst, Edward (2015) "Using the Chandrasekhar Recursions for Likelihood Evaluation of DSGE Models," Computational Economics, Vol. 45, pp. 693-705, DOI: http://dx.doi.org/10.1007/s10614-014-9430-2.

Herbst, Edward and Frank Schorfheide (2017) "Tempered Particle Filtering," Journal of Econometrics, forthcoming.

Iacoviello, Matteo and Stefano Neri (2010) "Housing market spillovers: evidence from an estimated DSGE model," American Economic Journal: Macroeconomics, Vol. 2 , pp. $125-164$.

Ioannides, Yannis M and Stuart S Rosenthal (1994) "Estimating the consumption and investment demands for housing and their effect on housing tenure status," Review of Economics and Statistics, pp. 127-141.

Jacquier, Eric, Nicholas G Polson, and Peter E Rossi (2002) "Bayesian analysis of stochastic volatility models," Journal of Business 86 Economic Statistics, Vol. 20, pp. 69-87, DOI: http://dx.doi.org/10.1198/073500102753410408.

Jarner, Søren Fiig and Ernst Hansen (2000) "Geometric ergodicity of Metropolis algorithms," Stochastic processes and their applications, Vol. 85, pp. 341-361, DOI: http://dx.doi.org/10.1016/S0304-4149(99)00082-4.

Jazwinski, Andrew H (1970) Stochastic processes and filtering theory: Academic Press. 
Jones, Galin L and James P Hobert (2001) "Honest exploration of intractable probability distributions via Markov chain Monte Carlo," Statistical Science, Vol. 16, pp. 312-334, URL: http://www.jstor.org.proxy.mul.missouri.edu/stable/ 3182773.

Julier, Simon J and Jeffrey K Uhlmann (1997) "A new extension of the Kalman filter to nonlinear systems," Int. symp. aerospace/defense sensing, simul. and controls, Vol. 3, pp. 182-193, DOI: http://dx.doi.org/10.1117/12.280797.

Justiniano, Alejandro and Giorgio E Primiceri (2008) "The time-varying volatility of macroeconomic fluctuations," American Economic Review, Vol. 98, pp. 604-41, DOI: http://dx.doi.org/10.1257/aer.98.3.604.

Justiniano, Alejandro, Giorgio E Primiceri, and Andrea Tambalotti (2010) "Investment shocks and business cycles," Journal of Monetary Economics, Vol. 57, pp. 132-145, DOI: http://dx.doi.org/10.1016/j.jmoneco.2009.12.008.

Katayama, Munechika and Kwang Hwan Kim (2016) "Uncertainty Shocks and the Relative Price of Investment Goods, Working Paper."

Kim, Jinill and Sunghyun Henry Kim (2003) "Spurious welfare reversals in international business cycle models," Journal of International Economics, Vol. 60, pp. 471-500, DOI: http://dx.doi.org/10.1016/S0022-1996(02)00047-8.

Kim, Jinill, Sunghyun Kim, Ernst Schaumburg, and Christopher A Sims (2008) "Calculating and using second-order accurate solutions of discrete time dynamic equilibrium models," Journal of Economic Dynamics and Control, Vol. 32, pp. 3397-3414, DOI: http://dx.doi.org/10.1016/j.jedc.2008.02.003.

Kim, Sangjoon, Neil Shephard, and Siddhartha Chib (1998) "Stochastic volatility: likelihood inference and comparison with ARCH models," The review of economic studies, Vol. 65, pp. 361-393, DOI: http://dx.doi.org/10.1111/1467-937X. 00050.

Kimball, Miles S (1990) "Precautionary Saving in the Small and in the Large," Econometrica, pp. 53-73.

King, Robert G, Charles I Plosser, and Sergio T Rebelo (1988a) "Production, growth and business cycles: I. The basic neoclassical model," Journal of Monetary Economics, Vol. 21, pp. 195-232, DOI: http://dx.doi.org/10.1016/0304-3932(88) 90030-X.

(1988b) "Production, growth and business cycles: II. New directions," Journal of Monetary Economics, Vol. 21, pp. 309-341, DOI: http://dx.doi.org/10. 1016/0304-3932(88) 90034-7.

Kiyotaki, Nobuhiro, John Moore et al. (1997) "Credit chains," Journal of Political Economy, Vol. 105, pp. 211-248. 
Leduc, Sylvain and Zheng Liu (2016) "Uncertainty shocks are aggregate demand shocks," Journal of Monetary Economics, Vol. 82, pp. 20-35, DOI: http://dx. doi.org/10.1016/j.jmoneco.2016.07.002.

Leland, Hayne E (1968) "Saving and uncertainty: The precautionary demand for saving," Quarterly Journal of Economics, Vol. 82, pp. 465-473.

Lester, Robert, Michael Pries, and Eric Sims (2014) "Volatility and welfare," Journal of Economic Dynamics and Control, Vol. 38, pp. 17-36.

Liu, Zheng, Pengfei Wang, and Tao Zha (2013) "Land-price dynamics and macroeconomic fluctuations," Econometrica, Vol. 81, pp. 1147-1184.

Lo, J (1972) "Finite-dimensional sensor orbits and optimal nonlinear filtering," IEEE Transactions on Information Theory, Vol. 18, pp. 583-588, DOI: http://dx.doi. org/10.1109/TIT.1972.1054885.

McLachlan, Geoffrey and David Peel (2004) Finite mixture models: John Wiley \& Sons.

Mendoza, Enrique G (1991) "Real business cycles in a small open economy," The American Economic Review, pp. 797-818.

Mengersen, Kerrie L and Richard L Tweedie (1996) "Rates of convergence of the Hastings and Metropolis algorithms," The annals of Statistics, Vol. 24, pp. 101121, DOI: http://dx.doi.org/10.1214/aos/1033066201.

Meyn, Sean P and Richard L Tweedie (2012) Markov chains and stochastic stability: Springer Science \& Business Media.

Miyamoto, Wataru and Thuy Lan Nguyen (2017) "Business cycles in small open economies: Evidence from panel data between 1900 and 2013," International Economic Review, Vol. 58, pp. 1007-1044.

Mora-Sanguinetti, Juan S and Margarita Rubio (2014) "Recent reforms in Spanish housing markets: An evaluation using a DSGE model," Economic Modelling, Vol. 44, pp. S42-S49.

Müller, Ulrich K (2013) "Risk of Bayesian inference in misspecified models, and the sandwich covariance matrix," Econometrica, Vol. 81, pp. 1805-1849, DOI: http: //dx.doi.org/10.3982/ECTA9097.

Mumtaz, Haroon and Konstantinos Theodoridis (2015) "The international transmission of volatility shocks: An empirical analysis," Journal of the European Economic Association, Vol. 13, pp. 512-533, DOI: http://dx.doi.org/10.1111/ jeea. 12120 .

(2017) "The Federal Reserve's implicit inflation target and macroeconomic dynamics: A SVAR analysis,"Technical report, Working Paper, School of Economics and Finance, Queen Mary University of London. 
Mumtaz, Haroon and Francesco Zanetti (2013) "The impact of the volatility of monetary policy shocks," Journal of Money, Credit and Banking, Vol. 45, pp. 535-558, DOI: http://dx.doi.org/10.1111/jmcb.12015.

Niemi, Jarad and Mike West (2010) "Adaptive mixture modeling Metropolis methods for Bayesian analysis of nonlinear state-space models," Journal of Computational and Graphical Statistics, Vol. 19, pp. 260-280, DOI: http://dx.doi .org/10.1198/ jcgs. 2010.08117.

Ninness, Brett and Soren Henriksen (2010) "Bayesian system identification via Markov chain Monte Carlo techniques," Automatica, Vol. 46, pp. 40-51, DOI: http://dx.doi.org/10.1016/j.automatica.2009.10.015.

Noh, Sanha (2018) "Posterior Inference on Parameters in a Nonlinear DSGE Model with an Application, Working Paper."

NøRgaard, Magnus, Niels K Poulsen, and Ole Ravn (2000) "New developments in state estimation for nonlinear systems," Automatica, Vol. 36, pp. 1627-1638, DOI: http://dx.doi.org/10.1016/S0005-1098(00)00089-3.

Oi, Walter Y (1961) "The desirability of price instability under perfect competition," Econometrica, pp. 58-64.

Omori, Yasuhiro, Siddhartha Chib, Neil Shephard, and Jouchi Nakajima (2007) "Stochastic volatility with leverage: Fast and efficient likelihood inference," Journal of Econometrics, Vol. 140, pp. 425-449, DOI: http://dx.doi.org/10.1016/ j.jeconom.2006.07.008.

Ortalo-Magné, François and Sven Rady (2002) "Tenure choice and the riskiness of non-housing consumption," Journal of Housing Economics, Vol. 11, pp. 266-279.

Owyang, Michael T, Jeremy Piger, and Howard J Wall (2005) "Business cycle phases in US states," Review of Economics and Statistics, Vol. 87, pp. 604-616.

Pitt, Michael K, Ralph dos Santos Silva, Paolo Giordani, and Robert Kohn (2012) "On some properties of Markov chain Monte Carlo simulation methods based on the particle filter," Journal of Econometrics, Vol. 171, pp. 134-151, DOI: http: //dx.doi.org/10.1016/j.jeconom.2012.06.004.

Primiceri, Giorgio E (2005) "Time varying structural vector autoregressions and monetary policy," The Review of Economic Studies, Vol. 72, pp. 821-852, DOI: http://dx.doi.org/10.1111/j.1467-937X.2005.00353.x.

Raitoharju, Matti and Simo Ali-Loytty (2012) "An adaptive derivative free method for Bayesian posterior approximation," IEEE Signal Processing Letters, Vol. 19, pp. 87-90, DOI: http://dx.doi.org/10.1109/LSP.2011.2179800. 
Raitoharju, Matti, Simo Ali-Löytty, and Robert Piché (2015) "Binomial Gaussian mixture filter," EURASIP Journal on Advances in Signal Processing, Vol. 2015, p. 36, DOI: http://dx.doi.org/10.1186/s13634-015-0221-2.

Roberts, Gareth O and Jeffrey S Rosenthal (1998) "Markov-chain monte carlo: Some practical implications of theoretical results," Canadian Journal of Statistics, Vol. 26, pp. 5-20, DOI: http://dx.doi.org/10.2307/3315667.

Roberts, Gareth O and Adrian FM Smith (1994) "Simple conditions for the convergence of the Gibbs sampler and Metropolis-Hastings algorithms," Stochastic processes and their applications, Vol. 49, pp. 207-216, DOI: http://dx.doi.org/ 10.1016/0304-4149(94)90134-1.

Roberts, Gareth O and Richard L Tweedie (1996) "Geometric convergence and central limit theorems for multidimensional Hastings and Metropolis algorithms," Biometrika, Vol. 83, pp. 95-110, DOI: http://dx.doi.org/10.1093/biomet/83. 1.95.

Rogerson, Richard (1988) "Indivisible labor, lotteries and equilibrium," Journal of monetary Economics, Vol. 21, pp. 3-16, DOI: http://dx.doi.org/10.1016/ 0304-3932 (88) 90042-6.

Rudebusch, Glenn D and Eric T Swanson (2012) "The bond premium in a DSGE model with long-run real and nominal," American Economic Journal: Macroeconomics, Vol. 4, pp. 105-143, URL: http://www .jstor .org.proxy.mul.missouri. edu/stable/41426391.

Ruge-Murcia, Francisco (2012) "Estimating nonlinear DSGE models by the simulated method of moments: With an application to business cycles," Journal of Economic Dynamics and Control, Vol. 36, pp. 914-938.

Runnalls, Andrew R (2007) "Kullback-Leibler approach to Gaussian mixture reduction," IEEE Transactions on Aerospace and Electronic Systems, Vol. 43, DOI: http://dx.doi.org/10.1109/TAES.2007.4383588.

Schmitt-Grohé, Stephanie and Martın Uribe (2003) "Closing small open economy models," Journal of international Economics, Vol. 61, pp. 163-185.

(2004) "Solving dynamic general equilibrium models using a second-order approximation to the policy function," Journal of Economic Dynamics and Control, Vol. 28, pp. 755-775, DOI: http://dx.doi.org/10.1016/S0165-1889(03) 00043-5.

Sims, Eric R (2011) "Permanent and transitory technology shocks and the behavior of hours: A challenge for DSGE models, Working Paper, University of Notre Dame."

Sinai, Todd and Nicholas S Souleles (2005) "Owner-occupied housing as a hedge against rent risk," Quarterly Journal of Economics, Vol. 120, pp. 763-789. 
Spiegelhalter, David J, Nicola G Best, Bradley P Carlin, and Angelika Van Der Linde (2002) "Bayesian measures of model complexity and fit," Journal of the Royal Statistical Society: Series B (Statistical Methodology), Vol. 64, pp. 583-639.

Strid, Ingvar and Karl Walentin (2009) "Block Kalman filtering for large-scale DSGE models," Computational Economics, Vol. 33, pp. 277-304, DOI: http://dx.doi. org/10.1007/s10614-008-9160-4.

Sun, Xiaojin and Kwok Ping Tsang (2017) "What Drives the Owner-Occupied and Rental Housing Markets? Evidence from an Estimated DSGE Model," Journal of Money, Credit and Banking, Vol. 49, pp. 443-468.

Tierney, Luke (1994) "Markov chains for exploring posterior distributions," The Annals of Statistics, Vol. 22, pp. 1701-1728, URL: http://www.jstor.org.proxy. mul.missouri.edu/stable/2242477.

Towbin, Pascal and Mr Sebastian Weber (2015) Price expectations and the US housing boom, Working Paper, No. 15-182: International Monetary Fund.

Uhlig, Harald (2005) "What are the effects of monetary policy on output? Results from an agnostic identification procedure," Journal of Monetary Economics, Vol. 52, pp. 381-419.

Uribe, Martin and Vivian Z Yue (2006) "Country spreads and emerging countries: Who drives whom?" Journal of international Economics, Vol. 69, pp. 6-36.

Van Der Merwe, Rudolph and Eric A Wan (2003) "Gaussian mixture sigma-point particle filters for sequential probabilistic inference in dynamic state-space models," Acoustics, Speech, and Signal Processing, 2003. Proceedings.(ICASSP'03). 2003 IEEE International Conference on, Vol. 6, pp. VI-701, DOI: http://dx.doi.org/ 10.1109/ICASSP. 2003.1201778.

Vats, Dootika, James M Flegal, and Galin L Jones (2017) "Multivariate output analysis for Markov chain Monte Carlo, Working Paper," DOI: http://dx.doi.org/ arXiv: 1512.07713.

Vihola, Matti (2012) "Robust adaptive Metropolis algorithm with coerced acceptance rate," Statistics and Computing, Vol. 22, pp. 997-1008, DOI: http://dx.doi.org/ $10.1007 / \mathrm{s} 11222-011-9269-5$.

Warne, Anders, Günter Coenen, and Kai Christoffel (2017) "Marginalized Predictive Likelihood Comparisons of Linear Gaussian State-Space Models with Applications to DSGE, DSGE-VAR, and VAR Models," Journal of Applied Econometrics, Vol. 32, pp. 103-119.

White, Halbert (1982) "Maximum likelihood estimation of misspecified models," Econometrica, Vol. 50, pp. 1-25, DOI: http://dx.doi.org/10.2307/1912526. 


\section{VITA}

Sanha Noh was born on December 14th, 1984 in Jeonju, South Korea. He earned a Bachelor's degree in Economics at Sung Kyun Kwan University in 2010. Then, under the supervision of Dr. Soosung Hwang at Sung Kyun Kwan University, he received a Master's degree in Economics in 2012.

In 2014, Sanha Noh entered graduate school to pursue a Ph.D. degree in Economics at the University of Missouri-Columbia. While at the University of MissouriColumbia, he was advised by Dr. Christopher Otrok and mainly focused on research topics in macroeconomics, especially business cycles, uncertainty, and policy analysis, with secondary interests in Bayesian macroeconometrics and asset pricing. During the Ph.D. degree, he worked for Dr. Christopher Otrok (University of Missouri) and Dr. Alessandro Rebucci (Johns Hopkins University) as a research assistant from 2015 to 2019. He is now working on a short-term project (from April 22, 2019 to June 21, 2019) for the International Monetary Fund (IMF) as a project officer.

He recently accepted a job offer from Korea Capital Market Institute as a research fellow. He is going to start his new job from July 1st, 2019 at Seoul, South Korea. 